REPORT ON THE U.S. GEOLOGICAL SURVEY'S EVALUATION PROGRAM FOR STANDARD REFERENCE SAMPLES DISTRIBUTED IN MAY 1995:

T-135 (TRACE CONSTITUENTS), M-134 (MAJOR CONSTITUENTS), N-45 (NUTRIENTS), N-46 (NUTRIENTS), P-24 (LOW IONIC STRENGTH), Hg-20 (MERCURY), AND SED-5 (BED MATERIAL)

by $H$. Keith Long and Jerry W. Farrar

U.S. GEOLOGICAL SURVEY

Open-File Report 95-395

Golden, Colorado

1995 
DEPARTMENT OF THE INTERIOR

BRUCE BABBITT, Secretary

U.S. GEOLOGICAL SURVEY

Gordon P. Eaton, Director

For additional information

write to:

William J. Shampine

U.S. Geological Survey

Water Resources Division, CR

Box 25046, Mail Stop 401

Denver Federal Center

Denver, CO 80225
Copies of this report can be purchased from:

U.S. Geological Survey

Books and Open-File Reports

Federal Center, Bldg. 810

Box 25425

Denver, CO 80225 


\section{CONTENTS}

Abstract
Introduction
Purpose and scope
Preparation of standard reference water samples
Laboratory analyses
Refatistical presentation of data
Refence

FIGURE

Figure 1. Statistical parameters shown on reported-data graphs

\section{TABLES}

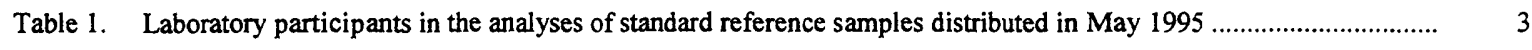

2. Analytes determined in standard reference samples distributed in May 1995......................................................... 7

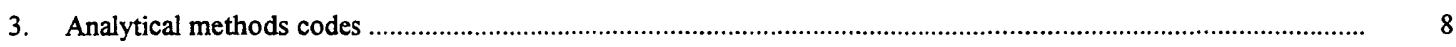

4. Overall laboratory performance ratings for standard reference water samples distributed in

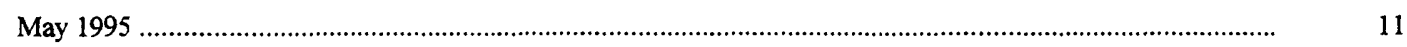

5. Laboratory performance ratings for standard reference water sample T-135 (trace constituents) ............................ 13

6. Laboratory performance ratings for standard reference water sample M-134 (major constituents) ........................... 21

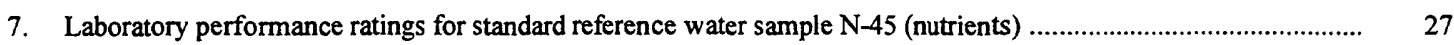

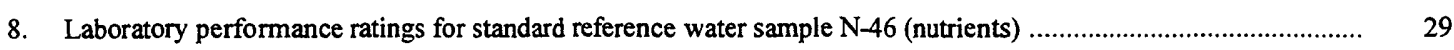

9. Laboratory performance ratings for standard reference water sample P-24 (low ionic strength) ............................. 31

10. Laboratory performance ratings for standard reference water sample $\mathrm{Hg}-20$ (mercury) ......................................... 33

11. Laboratory performance ratings for standard reference water sample SED-5 (bed material) .................................... 34

12. Statistical summary of reported data for standard reference water sample T-135 (trace constituents) ........................ 38

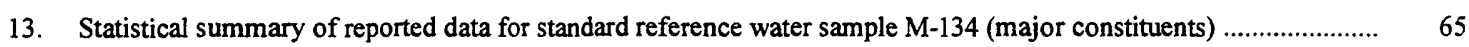

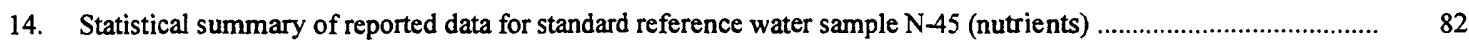

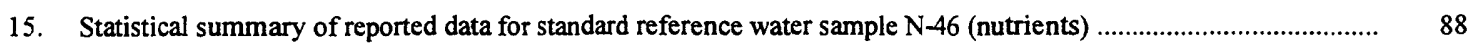

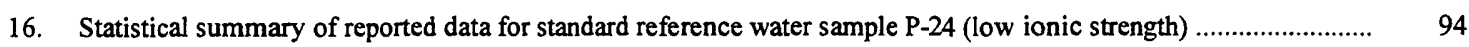

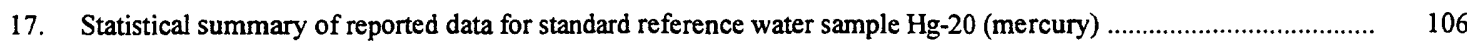

18. Statistical summary of reported data for standard reference water sample SED-5 (bed material) ................................ 108

19. Most probable values for constituents and properties in standard reference samples distributed in May 1995 


\title{
REPORT ON THE U.S. GEOLOGICAL SURVEY'S EVALUATION PROGRAM FOR STANDARD REFERENCE SAMPLES DISTRIBUTED IN MAY 1995: T-135 (TRACE CONSTITUENTS), M-134 (MAJOR CONSTITUENTS), N-45 (NUTRIENTS), N-46 (NUTRIENTS), P-24 (LOW IONIC STRENGTH),
}

\author{
Hg-20 (MERCURY), AND SED-5 (BED MATERIAL)
}

By H. Keith Long and Jerry W. Farrar

\begin{abstract}
This report presents the results of the U.S. Geological Survey's analytical evaluation program for 7 standard reference samples--T-135 (trace constituents), M-134 (major constituents), N-45 (nutrients), N-46 (nutrients), P-24 (low ionic strength), Hg-20 (mercury), Sed-5 (bed material)-that were distributed in May 1995 to 153 laboratories registered in the U.S. Geological Survey sponsored interlaboratory testing program. Analytical data that were received from 136 of the laboratories were evaluated with respect to: overall laboratory performance and relative laboratory performance for each analyte in the seven reference samples. Results of these evaluations are presented in tabular form. Also presented are tables and graphs summarizing the analytical data provided by each laboratory for each analyte in the seven standard reference samples. The most probable value for each analyte was determined using nonparametric statistics.
\end{abstract}

\section{INTRODUCTION}

The U.S. Geological Survey (USGS) conducts an interlaboratory evaluation program semiannually. This program provides a variety of reference materials to accomplish quality assurance testing of laboratories and to provide an adequate supply of samples that contribute to quality control programs of participating laboratories. Natural-matrix reference materials are preferred for use in this interlaboratory evaluation program. A series of samples are prepared and distributed each spring and fall. Occasionally, sediment samples are provided.

The program began in 1962 with a single sample containing major constituents that was prepared from distilled water and reagent grade chemicals. Twenty-three USGS laboratories participated in the 1962 determinations of six analytes in the major standard reference sample (SRS). Since that time, objectives of the program have been to:

(1) evaluate and improve the performance of USGS and other participating laboratories;

(2) provide a library of carefully prepared, homogeneous, stable reference materials for use in the quality control programs of laboratories;

(3) identify analytical problem areas;

(4) identify quality assurance needs with respect to environmental analyses and develop new reference materials to meet these needs; and

(5) ascertain the accuracy and precision of analytical methods. 
One hundred eighty-five USGS and non-USGS laboratories are registered in the program, which can currently provide eight standard reference sample types:

1. Trace constituents.

2. Major constituents.

3. Nutrients.

4. Low ionic strength.

5. Mercury.

6. Whole water (water with suspended sediment).

7. Acid mine drainage.

8. Sediment (bed material) for major and trace constituents.

When sufficient data are available, a most probable value is statistically determined for each analyte in the SRS.

Though this is not a laboratory certification program, participation in this continuing quality assurance program is mandatory for all laboratories providing water-analyses data for USGS data storage or use (publications). Federal, State, municipal, and university laboratories can participate even though they do not provide data to the USGS. Analyses of these SRS provides the means to alert participating laboratories of possible deficiencies in their analytical operations, and also provides reference materials for in-house quality control programs. Participating laboratories are identified only by a confidential code number.

A library of SRS, from previous evaluations, are available on request. Participating laboratories can request previous SRS for further testing, continuing quality assurance, and quality control programs by contacting:

Chief Laboratory Section

U.S. Geological Survey

Branch of Technical Development and Quality Systems

Denver Federal Center

Box 25046 MS 401

Denver, CO 80225-0046

\section{Purpose and Scope}

This report summarizes the analytical results submitted by 136 (table 1) of the 153 laboratories that requested and were shipped SRS for the May 1995 evaluation. Not all SRS are requested, nor necessarily analyzed by all the laboratories; nor do all laboratories enrolled in the program participate in each evaluation. Analytical results for the following, which were mailed the week of june 16,1995, are presented in this report:

$\begin{array}{ll}\text { T-135 } & \text { Trace constituents } \\ \text { M-134 } & \text { Major constituents } \\ \text { N-45 } & \text { Nutrients } \\ \text { N-46 } & \text { Nutrients } \\ \text { P-24 } & \text { Low ionic strength (precipitation) } \\ \text { Hg-20 } & \text { Mercury } \\ \text { Sed-5 } & \text { Bed material (sediment) }\end{array}$

The USGS requested that analytical results be returned by June 16, 1995 for evaluation and preparation of this report. This due date was extended until June 28, 1995. Each participating laboratory is requested to perform those determinations routinely made on the respective SRS for 
USGS investigations and to indicate the analytical method used to determine the concentration of each analyte. When analytical-method information was provided, it has been included in the respective data table. The analytical data are presented in ways that allow participants to evaluate data distribution, scatter, outliers, central tendency, bias, skewness, and method relationships.

Table 1.-Laboratory participants in the analyses of standard reference samples distributed in May 1995

\begin{tabular}{|c|c|c|}
\hline State & City & Participating Laboratory \\
\hline Alaska & Fairbanks & Alaska Department of Natural Resources \\
\hline Alabama & Tuscaloosa & Geological Survey of Alabama \\
\hline \multirow[t]{3}{*}{ Arizona } & Phoenix & Arizona Department of Health Services \\
\hline & Yuma & Burns and Roe Services Corporation \\
\hline & Yuma & Nestech \\
\hline Arkansas & Fayetteville & University of Arkansas \\
\hline \multirow[t]{12}{*}{ California } & Davis & University of California - Davis \\
\hline & La Verne & Metropolitan Water District of Southern California \\
\hline & Lakeside & Helix Water District \\
\hline & Oakland & East Bay Municipal Utility District \\
\hline & Riverside & University of California - Riverside \\
\hline & Sacramento & Anlab \\
\hline & Sacramento & US Bureau of Reclamation \\
\hline & Sacramento & USGS WRD \\
\hline & San Diego & USGS WRD \\
\hline & San Jacinto & Eastern Municipal Water District \\
\hline & Santa Fe Springs & West Coast Analytical Service, Inc. \\
\hline & West Sacramento & California Department of Water Resources \\
\hline \multirow[t]{15}{*}{ Colorado } & Arvada & Quanterra \\
\hline & Arvada & USGS National Water Quality Laboratory \\
\hline & Aurora & Core Laboratories, Inc. \\
\hline & Denver & Denver Water Department \\
\hline & Denver & Metro Wastewater Reclamation \\
\hline & Denver & USGS (Acid rain/Global climate change) \\
\hline & Denver & USGS (Earth Science Investigation Program) \\
\hline & Denver & USGS - Hydrologic Research Unit \\
\hline & Fort Collins & City of Fort Collins - Water Quality \\
\hline & Fort Collins & Colorado State University (Soil Testing Laboratory) \\
\hline & Fort Collins & US Department of Agriculture - Forest Service \\
\hline & Golden & EG \& G Rocky Flats \\
\hline & Loveland & Northern Colorado Water Conservation District \\
\hline & Northglenn & Northglenn Water Treatment Plant \\
\hline & Westminster & City of Westminster \\
\hline \multirow[t]{9}{*}{ Florida } & Brooksville & SW Florida Water Management District \\
\hline & Ocala & USGS WRD \\
\hline & Orlando & Post, Bucklye, Schuh, and Jernigan, Inc. \\
\hline & Ormond Beach & Environmental Laboratory \\
\hline & Palatka & St. John's River Management District \\
\hline & Tallahassee & City of Tallahassee \\
\hline & Tallahassee & Florida Department of Environmental Regulations \\
\hline & Tallahassee & Savannah Laboratories \\
\hline & Tampa & Hillsborough County Environmental Protection Commission \\
\hline \multirow[t]{5}{*}{ Georgia } & Athens & University of Georgia \\
\hline & Atlanta & Georgia Department of Natural Resources \\
\hline & Atlanta & USGS WRD \\
\hline & Decatur & Dekalb County Water Quality Laboratory \\
\hline & Tifton & United States Department of Agriculture \\
\hline
\end{tabular}


Table 1.--Laboratory participants in the analyses of standard reference samples distributed in May 1995-Continued

\begin{tabular}{|c|c|c|}
\hline & & \\
\hline Hawaii & Honolulu & Hawaii Institute of Geophysics \\
\hline Idaho & Boise & US Bureau of Reclamation \\
\hline \multirow[t]{3}{*}{ Illinois } & Champaign & Hazardous Waste Research Center \\
\hline & Champaign & Illinois Environmental Protection Agency \\
\hline & Chicago & Illinois Environmental Protection Agency \\
\hline Iowa & Des Moines & University Hygienic Laboratory, Des Moines Branch \\
\hline \multirow[t]{4}{*}{ Kansas } & Lawrence & Kansas Geological Survey \\
\hline & Topeka & City of Topeka \\
\hline & Topeka & Kansas Department of Health and Environment \\
\hline & Wichita & City of Wichita \\
\hline \multirow[t]{2}{*}{ Kentucky } & Lexington & Kentucky Geological Survey \\
\hline & Louisville & Metropolitan Sewer District \\
\hline \multirow[t]{2}{*}{ Maine } & Orono & University of Maine \\
\hline & Orono & Sawyer Environmental Center \\
\hline Maryland & Baltimore & Maryland Department of Health and Mental Hygiene \\
\hline Massachusetts & Wellesley Hills & Massachusetts Highway Department \\
\hline \multirow[t]{3}{*}{ Michigan } & Ann Arbor & University of Michigan - Department of Geological Science \\
\hline & Ann Arbor & University of Michigan \\
\hline & Detroit & Detroit Water and Sewerage Department \\
\hline \multirow[t]{4}{*}{ Minnesota } & Minneapolis & Braun Intertec Environmental, Inc. \\
\hline & Minneapolis & University of Minnesota, Department of Geology and Geophysics \\
\hline & St. Paul & Metro Waste Control Commission \\
\hline & St. Paul & University of Minnesota \\
\hline \multirow[t]{2}{*}{ Missouri } & Columbia & University of Missouri \\
\hline & Jefferson City & Missouri Department of Health \\
\hline \multirow[t]{2}{*}{ Montana } & Butte & Montana Bureau of Mines \& Geology \\
\hline & Helena & Department of Health and Environmental Sciences \\
\hline \multirow[t]{7}{*}{ Nevada } & Boulder City & US Bureau of Reclamation \\
\hline & Las Vegas & City of Las Vegas \\
\hline & Las Vegas & University of Nevada - Las Vegas \\
\hline & Reno & Desert Research Institute \\
\hline & Reno & Nevada State Health Laboratory \\
\hline & Reno & Reno-Sparks Wastewater Treatment \\
\hline & Sutcliffe & Pyramid Lake Fisheries \\
\hline New Mexico & Albuquerque & City of Albuquerque \\
\hline \multirow[t]{14}{*}{ New York } & Albany & USGS WRD \\
\hline & Brockport & State University of New York - Brockport \\
\hline & Buffalo & Erie County Laboratory \\
\hline & Grahamsville & New York City Department of Environmental Protection \\
\hline & Hempstead & Nassau County Department of Heal th \\
\hline & Ithaca & Cornell University, Agronomy Department \\
\hline & Milbrook & Institute of Ecosystem Studies \\
\hline & North Babylon & Ecotest Laboratories, Inc. \\
\hline & Oakdale & Suffolk County Water Authority \\
\hline & Rochester & Monroe County \\
\hline & Shokan & New York City Department of Environmental Protection \\
\hline & Syracuse & State University of New York - Syracuse \\
\hline & Valhalla & Department of Environmental Protection \\
\hline & Wantaugh & Cedar Creek Projects Laboratory \\
\hline \multirow[t]{2}{*}{ North Carolina } & Charlotte & Mecklenburg County \\
\hline & Durham & Duke University \\
\hline
\end{tabular}


Table 1.--Laboratory participants in the analyses of standard reference samples distributed in May 1995--Continued

\begin{tabular}{|c|c|c|}
\hline State & City & Participating Laboratory \\
\hline \multirow[t]{2}{*}{ North Carolina } & Durham & City of Durham \\
\hline & Greensboro & City of Greensboro \\
\hline \multirow[t]{2}{*}{ North Dakota } & Bismarck & North Dakota State Health Department \\
\hline & Bismarck & North Dakota State Water Commission \\
\hline \multirow[t]{4}{*}{ Ohio } & Cincinnati & US EPA \\
\hline & Cuyahoga Heights & Northeastern Ohio Regional Sewer District \\
\hline & Medina & Medina County Sanitary Engineering \\
\hline & Tiffin & Heidelberg College \\
\hline \multirow[t]{2}{*}{ Oklahoma } & Norman & Oklahoma Geological Survey \\
\hline & Oklahoma City & Oklahoma State Department of Health \\
\hline \multirow[t]{2}{*}{ Oregon } & Corvallis & US Department of Agriculture \\
\hline & Tigard & Unified Sewerage Agency \\
\hline \multirow[t]{2}{*}{ Pennsylvania } & Harrisburg & Pennsylvania Department of Environmental Resources \\
\hline & Somerset & Geochemical Testing \\
\hline Puerto Rico & San Juan & Department of Natural Resources \\
\hline \multirow[t]{3}{*}{ South Dakota } & Brookings & Northem Great Plains Laboratory \\
\hline & Brookings & SDSU - Water Quality Laboratory \\
\hline & Vermillion & South Dakota Geological Survey \\
\hline \multirow[t]{3}{*}{ Tennessee } & Chattanooga & TVA Environmental Chemistry \\
\hline & Jackson & Jackson Branch Laboratory \\
\hline & Knoxville & Cooperative Park Studies Unit \\
\hline \multirow[t]{2}{*}{ Texas } & College Station & Texas A \& M \\
\hline & Tyler & Analytical Testing Laboratories \\
\hline Vermont & Waterbury & Vermont Agency of Natural Resources \\
\hline \multirow[t]{3}{*}{ Virginia } & Culpepper & ESS Laboratories \\
\hline & Manassas & Occoquan Watershed Monitoring Laboratory \\
\hline & Richmond & Consolidated Laboratory Services \\
\hline Washington & Seattle & Brooks-Rand, Ltd. \\
\hline West Virginia & Morgantown & University of West Virginia \\
\hline \multirow[t]{3}{*}{ Wisconsin } & Madison & Madison Department of Public Health \\
\hline & Madison & University of Wisconsin \\
\hline & Milwaukee & Milwaukee Metro Sewerage District \\
\hline Wyoming & Laramie & Wyoming Department of Agriculture \\
\hline
\end{tabular}

\section{Preparation of Standard Reference Samples}

All of the SRS used in this evaluation were prepared by personnel of the USGS in Golden, Colo. and were analyzed for analyte concentrations and physical property values prior to mailing. A library of reference samples is maintained and can be requested by participating laboratories for use in their quality control programs.

Trace constituent sample T-135 was prepared using water collected from the Big Thompson River near Drake, Colorado. The water was pumped through 2- and $0.1-\mu \mathrm{m}$ filters, in series, into a 1300 $\mathrm{L}$ polypropylene drum. The water was continuously circulated and passed through a $0.1-\mu \mathrm{m}$ filter and ultraviolet sterilizer for 24 hours. Following this circulation, the water was acidified to $\mathrm{pH} 2$ with nitric acid and chlorinated to $5 \mathrm{ppm}$ free chlorine. The trace constituent concentrations were adjusted by adding reagent grade chemicals. The sample was circulated an additional 24 hours prior to bottling. During bottling the sample was pumped through an ultraviolet sterilizer and a $0.1-\mu \mathrm{m}$ filter. The $500-\mathrm{mL}$ polypropylene bottles used were acid leached and deionized-water rinsed, and autoclave sterilized 
Major constituent sample M-134 was prepared using tapwater from the Golden, Colorado potable water. The water was pumped through a $0.1-\mu \mathrm{m}$ filters into a $1300-\mathrm{L}$ polypropylene drum. The water was chlorinated to 5-ppm free chlorine with sodium hypochlorite, continuously circulated, and passed through a $0.1-\mu \mathrm{m}$ filter and ultraviolet sterilizer for 24 hours prior to bottling. During bottling the sample was pumped through an ultraviolet sterilizer and a $0.1-\mu \mathrm{m}$ filter. The $500-\mathrm{mL}$ polypropylene bottles used were acid leached, deionized-water rinsed, and autoclave sterilized.

Nutrient sample N-45 was prepared using 20 liters of deionized water. This sample was prepared the week prior to the mailing for this SRS evaluation. Desired concentrations were obtained by adding reagent grade chemicals. The sample was stirred 24 hours prior to bottling. The $12-\mathrm{mL}$ vials used were acid leached, deionized water rinsed, and autoclave sterilized. This sample is a concentrate which has to be diluted 10:100 prior to analysis.

Nutrient sample N-46 was prepared using water collected from the Fall River near Idaho Springs, Colorado. This sample was prepared the week prior to the mailing for this SRS evaluation. The water was pumped through 2- and $0.1-\mu \mathrm{m}$ filters, in series, into a $600-\mathrm{L}$ polypropylene drum and continuously circulated and passed through a $0.1-\mu \mathrm{m}$ filter for 48 hours. The desired nutrient concentrations were obtained by adding reagent-grade chemicals. The sample was circulated an additional 24 hours. The sample was continuously circulated for 24 hours prior to being bottled. The 250-mL polyethylene bottles used were new, amber, acid leached, and deionized-water rinsed.

Sample P-24 was prepared in a 400-L polypropylene drum using snow collected at Genesee Park, Colorado. The collected snow was allowed to melt; then it was pumped into the drum through 2and $0.1-\mu \mathrm{m}$ filters in series. Desired phosphate and fluoride concentrations were obtained by adding reagent-grade chemicals. Prior to bottling, the sample was continuously mixed for 48 hours while being circulated through a $0.1-\mu \mathrm{m}$ filter and an ultraviolet sterlizer. During bottling the sample was pumped through an ultraviolet sterilizer and a $0.1-\mu \mathrm{m}$ filter. The $500-\mathrm{mL}$ polypropylene bottles used were acid leached, deionized-water rinsed, and autoclave sterilized.

Sample Hg-20 was prepared using water collected from the Fall River near Idaho Springs, Colorado. The sample was prepared in a $190-\mathrm{L}$ polypropylene drum. The river water was pumped into this drum through $2-$ and $0.1-\mu \mathrm{m}$ filters in series. The water was continuously circulated and passed through a $0.1-\mu \mathrm{m}$ filter and ultraviolet sterilizer for 72 hours. Nitric acid (5-percent, v/v) and dichromate ion ( 0.05 -percent, $w / w)$ were added to stabilize the sample. The desired mercury concentration was obtained by adding a mercury standard solution. Following an additional 24 hours of circulation, the sample was bottled. The $125-\mathrm{mL}$ glass bottles and tetrafluoroethylene fluorocarbon resin caps used were new, acid leached, and deionized-water rinsed.

Sediment sample Sed-5 (bed material) was prepared from composited samples collected at Globeville, Colorado. Approximately 75 pounds of sediment was collected and composited. The washed sediment was dried. The material was then sieved through stainless steel sieves and the 125 to $300 \mu \mathrm{m}$ fractions were retained. These fractions were composited, well mixed, quartered and packaged in 20 -mL polyethylene vials as Sed-5. 


\section{LABORATORY ANALYSES}

The participating laboratories were asked to determine analytes which are summarized in table 2. The number of analytes varied from 26 in T-135 (trace constituents) to 1 in Hg-20 (mercury).

Table 2.--Analytes determined in standard reference samples distributed in May 1995

\begin{tabular}{|c|c|c|c|c|c|c|c|c|}
\hline \multicolumn{2}{|c|}{ Analyte or property } & Units & $T-135$ & M-134 & $N-45,46$ & P-24 & $\mathrm{Hg}-2 \mathrm{O}$ & Sed-5 \\
\hline Acidity & Acidity as $\mathrm{CaCO}_{3}$ & $\mathrm{mg} / \mathrm{L}$ & & & & $\mathrm{x}$ & & \\
\hline Alk & Alkalinity as $\mathrm{CaCO}_{3}$ & $\mathrm{mg} / \mathrm{L}$ & & $\mathrm{x}$ & & & & \\
\hline $\mathrm{Ag}$ & Silver & $\mu \mathrm{g} / \mathrm{L}$ & $\mathrm{x}$ & & & & & $\mathrm{x}$ \\
\hline Al & Aluminum & $\mu \mathrm{g} / \mathrm{L}$ & $\mathrm{x}$ & & & & & $\mathrm{x}$ \\
\hline As & Arsenic & $\mu \mathrm{g} / \mathrm{L}$ & $\mathrm{X}$ & & & & & $\mathrm{x}$ \\
\hline B & Boron & $\mu \mathrm{g} / \mathrm{L}$ & $\mathrm{X}$ & $\mathrm{x}$ & & & & $\mathrm{X}$ \\
\hline $\mathrm{Ba}$ & Barium & $\mu \mathrm{g} / \mathrm{L}$ & $\mathrm{x}$ & & & & & $\mathrm{x}$ \\
\hline $\mathrm{Be}$ & Beryllium & $\mu \mathrm{g} / \mathrm{L}$ & $\mathrm{x}$ & & & & & $\mathrm{X}$ \\
\hline $\mathrm{Ca}$ & Calcium & $\mathrm{mg} / \mathrm{L}$ & $\mathrm{x}$ & $\mathrm{x}$ & & $\mathrm{x}$ & & $\mathrm{X}$ \\
\hline $\mathrm{Cd}$ & Cadmium & $\mu \mathrm{g} / \mathrm{L}$ & $\mathrm{x}$ & & & & & $\mathrm{x}$ \\
\hline Co & Cobalt & $\mu \mathrm{g} / \mathrm{L}$ & $\mathrm{x}$ & & & & & $\mathrm{X}$ \\
\hline $\mathrm{Cr}$ & Chromium, total & $\mu \mathrm{g} / \mathrm{L}$ & $\mathrm{X}$ & & & & & $\mathrm{X}$ \\
\hline $\mathrm{Cu}$ & Copper & $\mu \mathrm{g} / \mathrm{L}$ & $\mathrm{x}$ & & & & & $\mathrm{x}$ \\
\hline DSRD & Dissolved solids & $\mathrm{mg} / \mathrm{L}$ & & $\mathrm{x}$ & & & & \\
\hline $\mathbf{F}$ & Fluoride & $\mathrm{mg} / \mathrm{L}$ & & $\mathrm{x}$ & & $\mathrm{X}$ & & \\
\hline $\mathrm{Fe}$ & Iron & $\mu \mathrm{g} / \mathrm{L}$ & $\mathrm{x}$ & & & & & $\mathrm{x}$ \\
\hline $\mathrm{Hg}$ & Mercury & $\mu \mathrm{g} / \mathrm{L}$ & & & & & $\mathrm{x}$ & \\
\hline $\mathrm{K}$ & Potassium & $\mathrm{mg} / \mathrm{L}$ & $\mathrm{x}$ & $\mathrm{x}$ & & $\mathrm{x}$ & & $\mathrm{x}$ \\
\hline$\underline{\mathrm{Li}}$ & Lithium & $\mu \mathrm{g} / \mathrm{L}$ & $\underline{x}$ & & & & & $\mathrm{x}$ \\
\hline $\mathrm{Mg}$ & Magnesium & $\mathrm{mg} / \mathrm{L}$ & $\mathrm{x}$ & $\mathrm{X}$ & & $\mathrm{x}$ & & $\mathrm{X}$ \\
\hline Mn & Manganese & $\mu \mathrm{g} / \mathrm{L}$ & $\mathrm{x}$ & & & & & $\mathrm{x}$ \\
\hline Mo & Molybdenum & $\mu \mathrm{g} / \mathrm{L}$ & $\mathrm{x}$ & & & & & $\mathrm{x}$ \\
\hline $\mathrm{Na}$ & Sodium & $\mathrm{mg} / \mathrm{L}$ & $\mathrm{x}$ & $\mathrm{x}$ & & $\mathrm{X}$ & & $\mathrm{x}$ \\
\hline $\mathrm{NH}_{3}$ as $\mathrm{N}$ & Ammonia & $\mathrm{mg} / \mathrm{L}$ & & & $\mathrm{x}$ & & & \\
\hline $\mathrm{NH}_{3}+\mathrm{Org} \mathrm{N}$ as $\mathrm{N}$ & Ammonia + Organic $\mathrm{N}$ & $\mathrm{mg} / \mathrm{L}$ & & & $\mathrm{x}$ & & & \\
\hline $\mathrm{Ni}$ & Nickel & $\mu \mathrm{g} / \mathrm{L}$ & $\mathrm{x}$ & & & & & $\mathrm{x}$ \\
\hline $\mathrm{NO}_{3}+\mathrm{NO}_{2}$ as $\mathrm{N}$ & Nitrate + Nitrite & $\mathrm{mg} / \mathrm{L}$ & & & $\mathrm{x}$ & & & \\
\hline $\mathrm{Pb}$ & Lead & $\mu \mathrm{g} / \mathrm{L}$ & $\mathrm{x}$ & & & & & $\mathrm{x}$ \\
\hline $\mathrm{pH}$ & & unit & & $\mathrm{X}$ & & $\mathrm{x}$ & & \\
\hline $\mathrm{PO}_{4}$ as $\mathrm{P}$ & Orthophosphate & $\mathrm{mg} / \mathrm{L}$ & & & $\mathrm{x}$ & & & \\
\hline total $P$ as $P$ & Phosphorus & $\mathrm{mg} / \mathrm{L}$ & & $\mathrm{X}$ & $\mathrm{x}$ & & & \\
\hline $\mathrm{Sb}$ & Antimony & $\mu \mathrm{g} / \mathrm{L}$ & $\mathrm{x}$ & & & & & $\mathrm{x}$ \\
\hline $\mathrm{Se}$ & Selenium & $\mu \mathrm{g} / \mathrm{L}$ & $\mathrm{X}$ & & & & & $\mathrm{x}$ \\
\hline $\mathrm{SiO}_{2}$ & Silica & $\mathrm{mg} / \mathrm{L}$ & $x$ & $\mathrm{X}$ & & & & $\mathrm{x}$ \\
\hline $\mathrm{SO}_{4}$ & Sulfate & $\mathrm{mg} / \mathrm{L}$ & & $\mathrm{x}$ & & & $\mathrm{x}$ & \\
\hline Sp Cond & Specific conductance & $\mu \mathrm{S} / \mathrm{cm}$ & & $\mathrm{x}$ & & & $\mathrm{x}$ & \\
\hline $\mathrm{Sr}$ & Strontium & $\mu \mathrm{g} / \mathrm{L}$ & $\mathrm{x}$ & $\mathrm{x}$ & & & & $\mathrm{x}$ \\
\hline $\mathrm{v}$ & Vanadium & $\mu \mathrm{g} / \mathrm{L}$ & $\mathrm{x}$ & $\mathrm{x}$ & & & & $\mathrm{x}$ \\
\hline$\underline{\mathrm{Zn}}$ & Zinc & $\mu \mathrm{g} / \mathrm{L}$ & $\mathrm{x}$ & & & & & $\mathrm{x}$ \\
\hline
\end{tabular}


Laboratories were requested to identify the method used for each analyte according to table 3 analytical method codes.

Table 3.-Analytical-method codes

\begin{tabular}{cl}
\hline Code & Method \\
\hline 0 & Other \\
1 & Atomic absorption: direct, air \\
2 & Atomic absorption: direct, nitrous oxide \\
3 & Atomic absorption: graphite furnace \\
4 & Inductively coupled plasma \\
5 & Direct current plasma \\
6 & Inductively coupled plasma/Mass spectrometry \\
7 & Ion chromatography \\
8 & Atomic absorption: cold vapor \\
10 & Atomic absorption: extraction [specify chelating agents ] \\
11 & Atomic absorption: hydride [specify reducing agent ] \\
12 & Flame emission \\
20 & Titration: colorimetric [specify color reagent ] \\
21 & Titration: electrometric [specify reducing or oxidizing agent/color reagent ] \\
22 & Colorimetric: [specify reducing or oxidizing agent/color reagent ] \\
40 & Ion selective electrode \\
41 & Electrometric [pH and Specific Conductance] \\
50 & Gravimetric: [specify filtration, evaporation, and so forth] \\
51 & Turbidimetric
\end{tabular}

Participating laboratories were also asked to use the references listed below to further define the methods.

1. American Public Health Association and others, 1992, Standard methods for the examination of water and wastewater 18th ed: Washington, D.C., American Public Health Association, 981p.

2. American Society for Testing and Materials, Annual book of ASTM standards: Philadelphia, v. 11.01, and v. 11.02.

3. Kopp, J.F., and McKee, G.F., 1979, Methods for chemical analysis of water and wastes: Cincinnati, U.S. Environmental Protection Agency, EPA 600/4-79-020, rev. 1983, $460 \mathrm{p}$.

4. Fishman, M.J., and Friedman, L.C., eds., 1989. Methods for determination of inorganic substances in water and fluvial sediments ( $3 \mathrm{~d}$ ed.): U.S. Geological Survey Techniques of Water-Resources Investigations, Book 5, Chapter A1, 545 p.

5. Miscellaneous manufacturer's instrument manuals or references. 


\section{LABORATORY PERFORMANCE RATINGS}

To facilitate interlaboratory performance comparisons, laboratory performance ratings, based on the analyses reported for each SRS, are included in tables 4 through 11 in this report. Averages of the analyte ratings and the number of analyte values reported for each SRS are given for each participating laboratory. Laboratory performance for each analyte is rated on a scale 4 to 0 , based on the absolute Z-value, as listed below:

\begin{tabular}{ll}
\hline Rating & Absolute Z-value \\
\hline 4 (Excellent) & 0.00 to 0.50 \\
3 (Good) & 0.51 to 1.00 \\
2 (Satisfactory) & 1.01 to 1.50 \\
1 (Questionable) & 1.51 to 2.00 \\
0 (Poor). & Greater than 2.00 \\
\hline
\end{tabular}

Overall laboratory performance ratings greater than 2.4 are considered satisfactory. Overall laboratory performance ratings between 2.0 and 2.39 are considered marginal; those less than 2.0 are considered poor.

\section{STATISTICAL PRESENTATION OF DATA}

Data in this report have been evaluated using nonparametric statistics as described by Hoaglin and others (1983). This statistical approach is a resistant statistic because the median is not influenced by outliers as is the mean in traditional statistics.

Analytical data for each analyte are presented in tabular and graphical forms in tables 11 through 18. Tabulated data for each analyte include the laboratory code number, reported values, analytical method, most probable value (MPV), number of reported values - excluding less than values (N), data range, $\mathrm{Z}$-value, and the $\mathrm{F}$-pseudosigma. (The $\mathrm{Z}$-value is equivalent to the $\mathrm{Z}$-score of traditional statistics, being the number of deviations the reported value is from the MPV. The Fpseudosigma is equivalent to the standard deviation $(\sigma)$ of traditional statistics when the data has a Gaussian distribution.) If an analyte has a sufficient number of determinations by a given method, usually 7 , the $\sigma$ for that analytical method is reported in the block of data listed for each analyte.

The median value is considered the MPV. Reported values of "less than" are used to establish the median, but are not considered in determining the data range. The median (midpoint) divides the ordered data into halves and is designated the MPV. The hinges include the middle 50-percent of the data and are the mid-values of the upper and lower halves of the data. (The hinges are similar to quartiles, but are not mathematically equivalent.) The range of data between the upper hinge (Hu) and the lower hinge ( $\mathrm{Hl})$, the hinge spread ( $\mathrm{H}$-spr), is used to calculate the F-pseudosigma, the laboratory performance rating, the upper warning level (UWL) and lower warning level (LWL), the upper control level (UCL) and the lower control level (LCL). The F-pseudosigma is calculated by comparison of the $\mathrm{H}$-spr value to the Gaussian distribution relation; 67.45 percent of the data "hinges" between plus and minus $1 \sigma$, resulting in a H-spr of $2 \times 0.6745=1.349 \sigma$, This relation allows the calculation of the F-pseudosigma $=(\mathrm{H}$-spr $) / 1.349$. Laboratories reporting "less than" values are not performance rated unless their reported "less than" values are greater than two Z-values from the MPV. 
The graphical plot of the reported data is shown in figure 1. The upper and lower boundaries of the graphical plots generally are +3 and -3 F-pseudosigma deviations from the median. (Computer-program scaling constraints do not permit these boundaries to always be graphed at exactly these values.) The graphical plot is a box plot/control chart with reported values grouped by analytical method in ascending order of value. Lines designate the MPV, $\mathrm{Hu}, \mathrm{Hl}$, and the (UWL) and (LWL) at +2 and -2 F-pseudosigma, respectively. "Less than" values are not plotted.

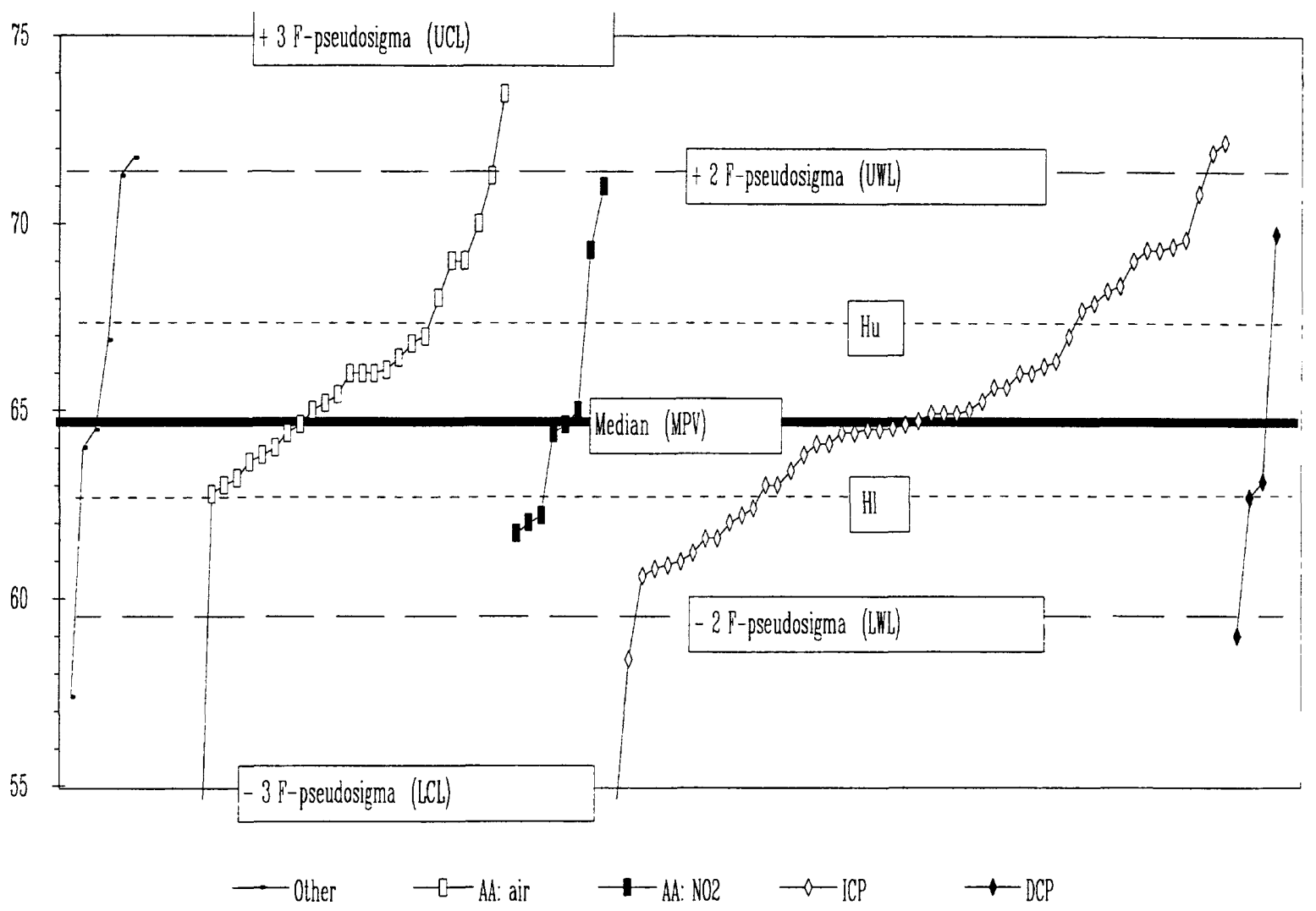

NOTE: vertical scale is the concentration value of the individual analyte in appropriate units (see table 2.) Methods shown are defined in Tables 3 and 10 through 17.

Figure 1.-Statistical parameters shown on reported-data graphs

\section{DISCUSSION}

The sediment used to prepare Sed-5 is from an abandoned smelter site. We consider the analytes available for transport to be of interest in this sample.

\section{REFERENCE}

Hoaglin, D.C., Mosteller, F., and Tukey, J.W., eds., 1983, Understanding Robust and Exploratory Data Analysis: John Wiley and Sons, Inc., 447p. 
Table 4. - Overall laboratory performance ratings for standard reference water samples distributed in May 1995

[Lab, laboratory number, OWR, overall weighted rating for all sample types; OLR, overall laboratory rating for reported values of a sample type;

V/90, number of reported values of 90 total possible values from all sample types; V/26, V/16, V/5,V/5,V/11,V/1,V26 are number of reported

values possible for T-135, M-134, N-45, N-46, P-24, $\mathrm{Hg}-20$, and SED-5 respectively]

\begin{tabular}{|c|c|c|c|c|c|c|c|c|c|c|c|c|c|c|c|c|}
\hline $\begin{array}{r}\text { Standard } \\
\text { Lab }\end{array}$ & $\begin{array}{c}\text { eference } \\
\text { OWR }\end{array}$ & $\begin{array}{l}\text { ple = } \\
\text { V/90 }\end{array}$ & $\begin{array}{l}\mathrm{T} 135 \\
\mathrm{OLR}\end{array}$ & U 26 & $\begin{array}{r}\text { M-134 } \\
\text { OLR }\end{array}$ & $V / 16$ & $\begin{array}{l}\mathrm{N}-45 \\
\mathrm{OLR}\end{array}$ & $v / 5$ & $\begin{array}{c}N-46 \\
\text { OLR }\end{array}$ & $\mathrm{V} / 5$ & PlR & v 11 & $\begin{array}{c}\mathrm{Hg}-20 \\
\text { OLR }\end{array}$ & $\mathrm{V} / 1$ & $\begin{array}{r}\text { SED } 5 \\
\text { OLR }\end{array}$ & $\mathrm{V} / 26$ \\
\hline 1 & 3.0 & 84 & 35 & 26 & 3.1 & 15 & 28 & $\begin{array}{l}4 \\
4\end{array}$ & 3.6 & 5 & 28 & 11 & 3 & 1 & 21 & 22 \\
\hline 2 & 2.0 & 2 & & & & & & & & & 20 & 2 & & & & \\
\hline 3 & 3.1 & 81 & 32 & 25 & 2.6 & 14 & 38 & 4 & 3.8 & 5 & 35 & 8 & 4 & 1 & 28 & 24 \\
\hline 4 & 0.1 & 18 & 00 & 16 & 0.5 & 2 & & & & & & & & & & \\
\hline 7 & 2.8 & 47 & 3,0 & 25 & 2.5 & 12 & 40 & 2 & 1.8 & 4 & 3,3 & 4 & & & & \\
\hline 9 & 2.8 & 21 & & & 2.8 & 13 & 30 & 5 & 2.7 & 3 & & & & & & \\
\hline 10 & 3.2 & 23 & 37 & 3 & 2.9 & 10 & 38 & 5 & 3.0 & 5 & & & & & & \\
\hline 11 & 2.1 & 44 & 21 & 20 & 2.1 & 13 & & & & & 20 & 10 & 4 & 1 & & \\
\hline 12 & 2.7 & 7 & & & & & 3,3 & 3 & 2.3 & 4 & & & & & & \\
\hline 13 & 2.6 & 62 & 22 & 22 & 2.3 & 12 & 13 & 4 & 2.5 & 4 & & '् & 3 & 1 & 3.4 & 19 \\
\hline 15 & 3.1 & 78 & 30 & 24 & 2.4 & 12 & 35 & 4 & 3.4 & 5 & 24 & 7 & 4 & 1 & 36 & 25 \\
\hline 16 & 2.4 & 45 & 29 & 21 & 2.6 & 13 & 08 & 5 & 1.6 & 5 & & & 2 & 1 & & \\
\hline 18 & 3.2 & 73 & 33 & 23 & 3.1 & 15 & 32 & 5 & 4.0 & 5 & & 's & 4 & 1 & 3.0 & 24 \\
\hline 19 & 2.3 & 26 & 18 & 11 & 2.7 & 10 & 20 & 3 & 3.5 & 2 & & & & & & \\
\hline 21 & 3.8 & 6 & 3,0 & । & & & 40 & 5 & & & & से & & & & \\
\hline 22 & 3.5 & 2 & & & & & 40 & 1 & 3.0 & 1 & & 3 & & & & \\
\hline 23 & 2.5 & 41 & 19 & 8 & 2.7 & 6 & 2.3 & 4 & 3.8 & 5 & 30 & 6 & & & 22 & 12 \\
\hline 24 & 2.7 & 39 & 26 & 25 & 3.0 & 13 & & & & & & & 1 & 1 & & \\
\hline 25 & 1.5 & 49 & 14 & 21 & 1.4 & 14 & 20 & 2 & 1.3 & 3 & 21 & 9 & & & & \\
\hline 26 & 3.0 & 42 & 31 & 16 & 3.1 & 11 & 23 & 3 & 2.3 & 3 & 3,0 & 9 & & & & \\
\hline 27 & 1.6 & 9 & 10 & 3 & 1.6 & 5 & & & & & 30 & 1 & & & & \\
\hline 28 & 1.6 & 73 & 18 & 23 & 1.3 & 15 & 10 & 5 & 2.8 & 5 & 24 & 7 & & & 10 & 18 \\
\hline 30 & 3.3 & 33 & 34 & 20 & 3.3 & 4 & & & 1.5 & 2 & & & & & 36 & ) \\
\hline 32 & 2.1 & 65 & 28 & 24 & 2.6 & 14 & 05 & 2 & & & & 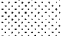 & 3 & 1 & 13 & 24 \\
\hline 33 & 3.4 & 32 & 29 & 10 & 3.8 & 11 & 40 & 1 & 1.0 & 1 & 36 & 9 & & & & \\
\hline 34 & 3.7 & 3 & 35 & 2 & & & & & & & & & 4 & 1 & & \\
\hline 35 & 4.0 & 3 & 40 & 3 & & & & & & & & ह & & & & \\
\hline 36 & 1.4 & 50 & 13 & 22 & 1.4 & 14 & 2,3 & 4 & 0.0 & 5 & 33 & 4 & 1 & 1 & & \\
\hline 38 & 3.4 & 24 & & & 3.5 & 8 & 3,8 & 5 & 2.8 & 5 & 33 & 6 & & & & \\
\hline 39 & 2.5 & 50 & 25 & 22 & 2.6 & 14 & 30 & 4 & & & 22 & 9 & 3 & 1 & & \\
\hline 40 & 3.3 & 35 & 35 & 21 & 3.1 & 14 & & & & & & B & & & & \\
\hline 42 & 3.3 & 47 & 36 & 25 & 3.3 & 15 & 20 & 3 & 3.3 & 3 & & & 2 & 1 & & \\
\hline 43 & 3.2 & 20 & 37 & 2 & 3.2 & 11 & 100 & 1 & 2.0 & 1 & & & & & & \\
\hline 45 & 1.8 & 6 & 20 & 5 & & & & & & & & 's & 1 & 1 & & \\
\hline 46 & 3.0 & 43 & 29 & 19 & 2.6 & 8 & 38 & 5 & 3.6 & 5 & 26 & 5 & 4 & 1 & & \\
\hline 48 & 2.2 & 77 & 23 & 22 & 2.3 & 12 & 12 & 5 & 1.4 & 5 & 13 & 9 & 0 & 1 & 30 & 23 \\
\hline 50 & 2.8 & 30 & 24 & 16 & 3.5 & 13 & & & & & & है & 2 & 1 & & \\
\hline 52 & 3.2 & 77 & 34 & 24 & 3.3 & 14 & 38 & 5 & 2.2 & 5 & 31 & 1 & 4 & 1 & 32 & 21 \\
\hline 53 & 2.7 & 6 & & & & & 27 & 3 & 2.7 & 3 & & & & & & \\
\hline 54 & 3.3 & 17 & 35 & 6 & 3.2 & 11 & & & & & & & & & & \\
\hline 55 & 2.9 & 45 & 28 & 23 & 2.6 & 14 & 32 & 5 & 3.5 & 2 & & & 4 & 1 & & \\
\hline 56 & 2.1 & 17 & & & 2.0 & 9 & 23 & 4 & 2.0 & 4 & & & & & & \\
\hline 57 & 2.1 & 17 & & & 2.4 & 12 & & & 1.4 & 5 & & से & ' & & & \\
\hline 58 & 1.7 & 69 & 16 & 18 & 1.5 & 12 & 18 & 5 & 0.0 & 5 & 17 & 11 & 0 & 1 & 23 & 17 \\
\hline 59 & 3.0 & 50 & 32 & 22 & 2.5 & 13 & 2.4 & 5 & 3.2 & 5 & 34 & 5 & & & & औ \\
\hline 60 & 2.8 & 19 & 27 & 11 & & & 2,0 & 3 & 3.6 & 5 & & & & & & \\
\hline 64 & 3.3 & 33 & 30 & 6 & 3.1 & 10 & 40 & 4 & 3.5 & 4 & 33 & 9 & & & & \\
\hline 68 & 1.8 & 53 & 10 & 25 & 1.7 & 11 & & 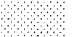 & 2.8 & 4 & & & & & 29 & 13 \\
\hline 69 & 3.3 & 35 & 32 & 19 & 3.6 & 10 & 40 & 1 & 2.0 & 1 & & & 4 & 1 & 23 & 3 \\
\hline 70 & 3.2 & 46 & 32 & 23 & 3.5 & 13 & 3.3 & 4 & 3.0 & 5 & & & 2 & 1 & & \\
\hline 73 & 3.0 & 9 & 30 & 9 & & & & & & & & & & & & \\
\hline 75 & 3.6 & 41 & 35 & 22 & 3.7 & 10 & 35 & 4 & 3.5 & 4 & & & 4 & 1 & & \\
\hline 76 & 3.3 & 23 & 3,1 & 11 & 3.6 & 7 & 30 & 2 & 3.5 & 2 & & & 4 & 1 & & \\
\hline 80 & 2.0 & 26 & 29 & 8 & 1.8 & 12 & 20 & 3 & 1.0 & 3 & & & & & & \\
\hline 81 & 2.5 & 68 & 22 & 23 & 3.1 & 13 & & & & & 13 & 10 & 2 & 1 & 30 & 21 \\
\hline 83 & 3.0 & 30 & 34 & 16 & 2.5 & 8 & 27 & 3 & 3.0 & 3 & & & & & & \\
\hline 84 & 2.6 & 17 & 28 & 5 & 3.1 & 7 & 30 & 2 & 1.0 & 3 & & & & & & \\
\hline 85 & 3.3 & 47 & 31 & 23 & 3.4 & 14 & 3.6 & 5 & 3.2 & 5 & & & & & & \\
\hline 86 & 3.0 & 41 & 34 & 21 & 2.9 & 11 & 25 & 4 & 2.3 & 4 & & & 0 & 1 & & \\
\hline 87 & 2.5 & 40 & 24 & 18 & 3.0 & 11 & 20 & 5 & 2.8 & 5 & & & 0 & 1 & & \\
\hline 88 & 1.0 & 3 & & & & & 10 & 3 & & & & & & & & \\
\hline 89 & 2.9 & 57 & 25 & 22 & 3.4 & 13 & 3.4 & 5 & 3.2 & 5 & 27 & 11 & 4 & 1 & & \\
\hline 90 & 2.8 & 19 & 18 & 4 & 3.6 & 5 & 3.2 & 5 & 2.6 & 5 & & & & & & \\
\hline 91 & 2.8 & 12 & 35 & 2 & & & 32 & 5 & 2.0 & 5 & & & & & & \\
\hline 92 & 1.8 & 40 & 15 & 13 & 2.3 & 10 & $1 / 3$ & 4 & 2.0 & 4 & 15 & 8 & 3 & 1 & & \\
\hline 93 & 2.7 & 10 & & से & 1.3 & 3 & 4.0 & 1 & 4.0 & 1 & 30 & 5 & & & & \\
\hline 94 & 3.7 & 10 & & \& & 4.0 & 2 & 3.3 & 4 & 4.0 & 4 & & & & & & \\
\hline 96 & 3.1 & 29 & 31 & 11 & 2.4 & 7 & 3.6 & 5 & 3.2 & 5 & & & 4 & 1 & & \\
\hline 97 & 2.8 & 48 & 25 & 24 & 2.5 & 13 & 30 & 5 & 4.0 & 5 & & 3 & 3 & 1 & & \\
\hline 100 & 2.4 & 68 & 23 & 25 & 2.3 & 7 & 2,3 & 3 & 2.7 & 3 & 30 & 3 & 4 & 1 & 2.5 & 26 \\
\hline
\end{tabular}


Table 4. - Overall laboratory performance ratings for standard reference water samples distributed in May 1995 Continued

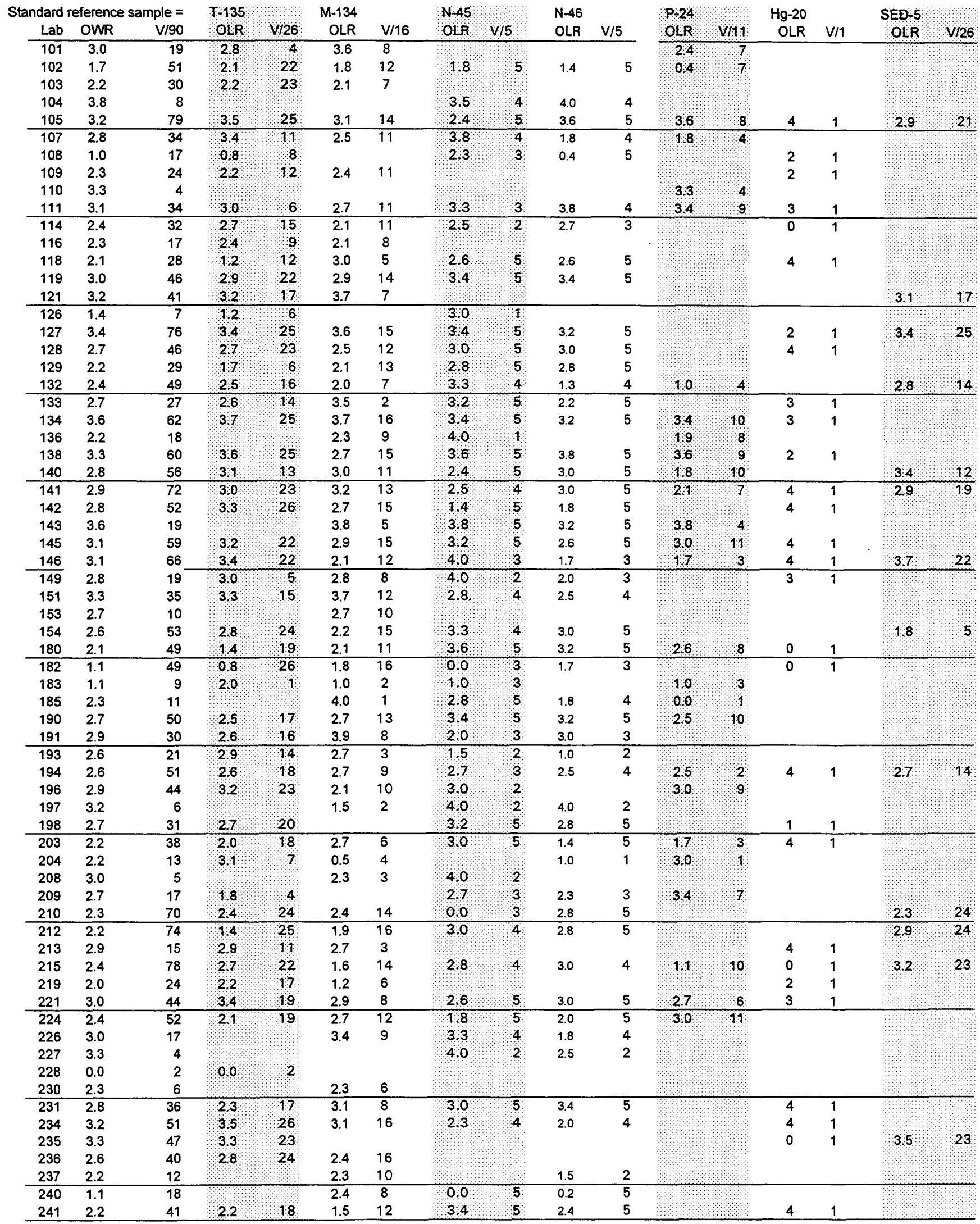


Table 5. -Laboratory performance ratings for standard reference water sample $T-135$ (trace constituents)

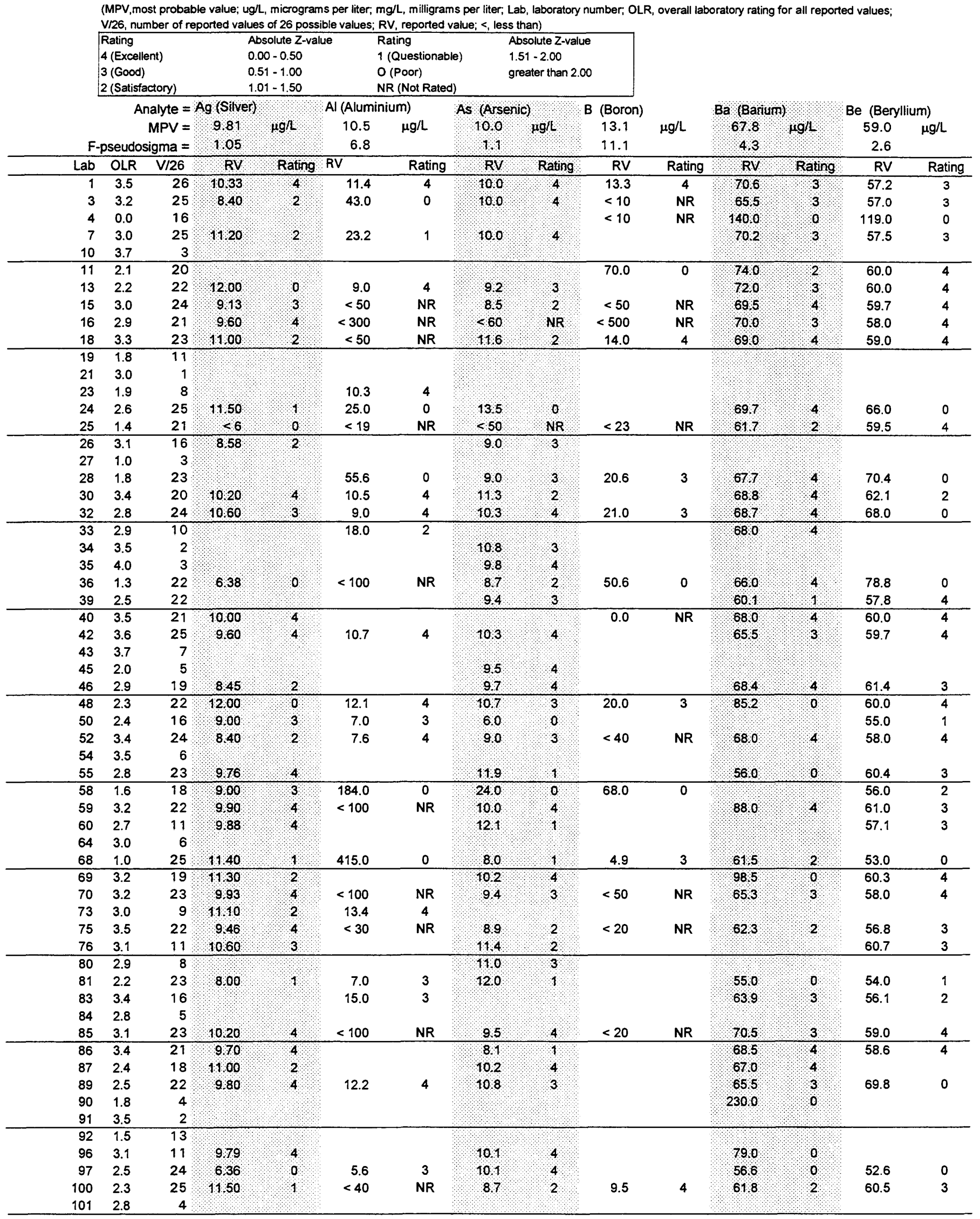


Table 5. -Laboratory performance ratings for standard reference water sample T-135 (trace constituents) --Continued

\begin{tabular}{|c|c|c|c|c|c|c|c|c|c|c|c|c|c|c|}
\hline \multicolumn{5}{|c|}{ Analyte $=A g($ Silver $)$} & \multicolumn{2}{|c|}{ Al (Aluminium) } & \multicolumn{2}{|c|}{ As (Arsenic) } & \multicolumn{2}{|l|}{ B (Boron) } & \multicolumn{2}{|c|}{ Ba (Barium) } & \multicolumn{2}{|c|}{ Be (Beryllium) } \\
\hline & & MPV $=$ & 981 & $19 /$ & 10.5 & $\mu \mathrm{g} / \mathrm{L}$ & 100 & нg/4 & 13.1 & $\mu \mathrm{g} / \mathrm{L}$ & $\begin{array}{r}678 \\
\end{array}$ & $1,9 /$ & & $\mu g / L$ \\
\hline \multicolumn{3}{|c|}{ F.pseudosigma $=$} & 105 & & 6.8 & & 12 & & 11.1 & & 4,3 & & 2.6 & \\
\hline Lab & OLR & $V / 26$ & $R V$ & Rating & $\mathrm{RV}$ & Rating & $\mathrm{RV}$ & Rating & RV & Rating & $\mathrm{RV}$ & Rating & RV & Rating \\
\hline 102 & 2.1 & 22 & 880 & 3 & 7.9 & 4 & 220 & 0 & & & 590 & 13 & 59.0 & 4 \\
\hline 103 & 2.2 & 23 & $<5$ & 0 & $<30$ & NR & $\mathbf{1} 10$ & NR & 8.0 & 4 & 610 & 1 & 57.0 & 3 \\
\hline 105 & 3.5 & 25 & 8.90 & 3 & 10.3 & 4 & 1014 & 4 & & & 672 & 4 & 57.0 & 3 \\
\hline 107 & 3.4 & 11 & 027 & 3 & 9.0 & 4 & & & & & & & & \\
\hline 108 & 0.8 & 8 & 620 & 0 & & & 80 & 1 & & & & & & \\
\hline 109 & 2.2 & 12 & & & & & 104 & 4 & & & & & & \\
\hline 111 & 3.0 & 6 & & & 10.2 & 4 & & & & & & & & \\
\hline 114 & 2.7 & 15 & 1000 & 4 & & & & & & & & & 58.0 & 4 \\
\hline 116 & 2.4 & 9 & & & & & & & $<30$ & NR & 72,0 & 3 & & \\
\hline 118 & 1.2 & 12 & 890 & 3 & & & 147 & 0 & & & & & & \\
\hline 119 & 2.9 & 22 & 970 & 4 & 10.5 & 4 & 100 & 4 & 10.0 & 4 & 730 & 2 & 53.9 & 1 \\
\hline 121 & 3.2 & 17 & & & 40.0 & 0 & & & 21.0 & 3 & 680 & 4 & & \\
\hline 126 & 1.2 & 6 & & & & & $10 \%$ & 3 & & & & & & \\
\hline 127 & 3.4 & 25 & 863 & 2 & 8.3 & 4 & 10,4 & 4 & $<15$ & NR & 66,4 & 4 & 57.5 & 3 \\
\hline 128 & 2.7 & 23 & 987 & 4 & 7.6 & 4 & 96 & 4 & $<10$ & NR & 601 & 1 & 58.2 & 4 \\
\hline 129 & 1.7 & 6 & & & & & & & 105.0 & 0 & & & & \\
\hline 132 & 2.5 & 16 & & & 41.0 & 0 & & & 9.0 & 4 & & & & \\
\hline 133 & 2.6 & 14 & 985 & 4 & & & 10,1 & 4 & & & 683 & 4 & 64.0 & 1 \\
\hline 134 & 3.7 & 25 & 1000 & 4 & 10.1 & 4 & 100 & 4 & 11.6 & 4 & 658 & 4 & 57.6 & 3 \\
\hline 138 & 3.6 & 25 & 990 & 4 & 9.5 & 4 & 92 & 3 & 9.8 & 4 & 70,5 & 3 & 59.0 & 4 \\
\hline 140 & 3.1 & 13 & & & & & & & & & & & & \\
\hline 141 & 3.0 & 23 & 1000 & 4 & 14.0 & 3 & $<50$ & NR & 30.0 & 1 & 730 & 2 & 61.0 & 3 \\
\hline 142 & 3.3 & 26 & 1026 & 4 & 8.0 & 4 & 96 & 4 & 10.8 & 4 & 692 & 4 & 58.2 & 4 \\
\hline 145 & 3.2 & 22 & & & 17.1 & 3 & 147 & 0 & $<2.4$ & NR & 667 & 4 & 59.1 & 4 \\
\hline 146 & 3.4 & 22 & 1090 & 2 & 35.6 & 0 & B 5 & 2 & & & 66,6 & 4 & 58.5 & 4 \\
\hline 149 & 3.0 & 5 & 970 & 4 & & & & & & & 700 & 3 & & \\
\hline 151 & 3.3 & 15 & $<10$ & NR & 9.0 & 4 & 87 & 2 & & & & & & \\
\hline 154 & 2.8 & 24 & 10,20 & 4 & 8.4 & 4 & 101 & 4 & 14.5 & 4 & 621 & 2 & 60.0 & 4 \\
\hline 180 & 1.4 & 19 & 1260 & 0 & $<36.5$ & NR & $<334$ & $\mathrm{NR}$ & $<10$ & NR & 722 & 2 & 60.9 & 3 \\
\hline 182 & 0.8 & 26 & $\mathbf{1 1 5}$ & 0 & 25.5 & 0 & 8.5 & 2 & 28.9 & 2 & 54.2 & 0 & 31.0 & 0 \\
\hline 183 & 2.0 & 1 & & & & & & & & & & & & \\
\hline 190 & 2.5 & 17 & 9,94 & 4 & 7.7 & 4 & 100 & 4 & & & & & & \\
\hline 191 & 2.6 & 16 & & & 17.0 & 3 & & & & & 64.0 & 3 & & \\
\hline 193 & 2.9 & 14 & 9.80 & 4 & & & 80 & 1 & & & 640 & 3 & & \\
\hline 194 & 2.6 & 18 & 10,00 & 4 & $<500$ & NR & 100 & 4 & $<100$ & NR & $\begin{array}{r}100 \\
0\end{array}$ & NR & 675.0 & 0 \\
\hline 196 & 3.2 & 23 & 8.88 & 3 & 9.5 & 4 & 10,5 & 4 & & & 678 & 4 & 56.8 & 3 \\
\hline 198 & 2.7 & 20 & 1100 & 2 & 8.2 & 4 & 116 & 2 & & & 822 & 0 & 62.4 & 2 \\
\hline 203 & 2.0 & 18 & $\begin{array}{l}700 \\
\end{array}$ & 0 & 4.9 & 3 & 93 & 3 & & & 763 & 1 & & \\
\hline 204 & 3.1 & 7 & & & & & & & & & & & & \\
\hline 209 & 1.8 & 4 & & - & $<27$ & NR & & & & & & & & \\
\hline 210 & 2.4 & 24 & 14.50 & 0 & $<0.2$ & NR & 20.5 & 0 & 12.0 & 4 & 63.5 & 3 & 59.5 & 4 \\
\hline 212 & 1.4 & 25 & 1200 & o & $<200$ & NR & 99 & 4 & 13.0 & 4 & 790 & 0 & 61.0 & 3 \\
\hline 213 & 2.9 & 11 & 8.80 & 3 & & & 117 & 1 & & & & & 67.0 & 0 \\
\hline 215 & 2.7 & 22 & 3,35 & 0 & $<40$ & NR & 140 & 0 & 57.0 & 0 & 660 & 4 & 56.0 & 2 \\
\hline 219 & 2.2 & 17 & & & 8.0 & 4 & 96 & 4 & & & & & 57.0 & 3 \\
\hline 221 & 3.4 & 19 & 9.51 & 4 & 14.1 & 3 & 9.9 & 4 & & & 675 & 4 & & \\
\hline 224 & 2.1 & 19 & & & 5.9 & 3 & 94 & 3 & & & 245 & 0 & 56.1 & 2 \\
\hline 228 & 0.0 & 2 & & & 130.0 & 0 & & & & & & & & \\
\hline 231 & 2.3 & 17 & 5.38 & 0 & & & 106 & 3 & & & 797 & 0 & & \\
\hline 234 & 3.5 & 26 & 9.81 & 4 & 16.0 & 3 & 102 & 4 & 11.0 & 4 & 677 & 4 & 59.7 & 4 \\
\hline 235 & 3.3 & 23 & 10,30 & 4 & 13.7 & 4 & 113 & 2 & 10.0 & 4 & 670 & 4 & 58.5 & 4 \\
\hline 236 & 2.8 & 24 & 600 & 0 & 44.0 & 0 & 480 & 0 & 0.0 & NR & 650 & 3 & 58.0 & 4 \\
\hline 241 & 2.2 & 18 & 904 & 3 & & & Q 2 & 3 & & & & & 22.0 & 0 \\
\hline & 1.4 & & & & & & & & & & & & & \\
\hline
\end{tabular}


Table 5. -Laboratory performance ratings for standard reference water sample T-135 (trace constituents)

\section{-.-Continued}

(MPV,most probable value; ug/ $\mathrm{L}$, micrograms per liter; $\mathrm{mg} / \mathrm{L}$, milligrams per liter; Lab, laboratory number; OLR, overall laboratory rating for all reported values:

V/26, number of reported values of 26 possible values; RV, reported value; <, less than)

\begin{tabular}{|llll} 
Rating & Absolute Z-value & Rating & Absolute Z-value \\
4 (Excellent) & $0.00-0.50$ & 1 (Questionable) & $1.51-2.00$ \\
3 (Good) & $0.51-1.00$ & $O$ (Poor) & greater than 2.00 \\
2 (Satisfactory) & $1.01-1.50$ & NR (Not Rated) &
\end{tabular}

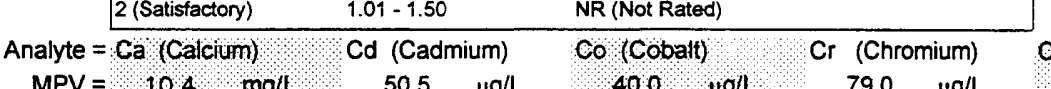

$620 \mathrm{pg} / \mathrm{L} \quad 228 \mu \mathrm{g} / \mathrm{L} \quad 0.96 \mathrm{mg} / \mathrm{l}$

\begin{tabular}{|c|c|c|c|c|c|c|c|c|c|c|c|c|c|c|}
\hline$a-$ & 0.6 & & 3.2 & & 2,6 & & 5.5 & & 42 & & 11 & & 009 & \\
\hline Lab & $\mathrm{RV}$ & Rating & RV & Rating & $\mathrm{RV}$ & Rating & RV & Rating & $\mathrm{RV}$ & Rating & RV & Rating & RV & Rating \\
\hline 1 & 108 & 3 & 49.1 & 4 & 396 & 4 & 80.9 & 4 & 619 & 4 & 235 & 3 & 090 & 3 \\
\hline 3 & 102 & 4 & 48.6 & 3 & 410 & 4 & 81.0 & 4 & 650 & 3 & 250 & 1 & 102 & 3 \\
\hline 4 & 22.3 & 0 & & & 620 & 0 & 139.0 & 0 & 1240 & 0 & 465 & 0 & & \\
\hline 7 & 104 & 4 & 57.0 & 1 & 398 & 4 & 83.6 & 3 & 608 & 4 & 220 & 3 & 127 & 0 \\
\hline 10 & & & & & & & & & & & 230 & 4 & & \\
\hline 11 & 94 & 1 & 50.0 & 4 & 400 & 4 & 90.0 & 1 & 600 & 4 & 250 & 1 & 0.94 & 4 \\
\hline 13 & 115 & 1 & 53.0 & 3 & 420 & 3 & 86.0 & 2 & 700 & 1 & 238 & 3 & 080 & 1 \\
\hline 15 & 10,8 & 3 & 51.3 & 4 & 427 & 2 & 82.0 & 3 & 645 & 3 & 222 & 3 & 091 & 3 \\
\hline 16 & 102 & 4 & 53.0 & 3 & 38.5 & 3 & 70.0 & 1 & 615 & 4 & 211 & 1 & 110 & 1 \\
\hline 18 & 10.6 & 4 & 53.0 & 3 & 420 & 3 & 79.0 & 4 & 630 & 4 & 228 & 4 & 0.90 & 3 \\
\hline 19 & 108 & 3 & 55.0 & 2 & & & 86.0 & 2 & 690 & 1 & 241 & 2 & 084 & 2 \\
\hline 21 & & & & & & & & & & & 220 & 3 & & \\
\hline 23 & 8,6 & 0 & & & & & & & 56.4 & 2 & & & & \\
\hline 24 & 102 & 4 & 48.0 & 3 & 402 & 4 & 75.8 & 3 & 650 & 3 & 218 & 3 & 094 & 4 \\
\hline 25 & 9.6 & 2 & 51.0 & 4 & 320 & 0 & 66.0 & 0 & 560 & 2 & 201 & 0 & $<21$ & NR \\
\hline 26 & 106 & 4 & 51.0 & 4 & & & 81.2 & 4 & 635 & 4 & 228 & 4 & 116 & 0 \\
\hline 27 & 139 & 0 & & & & & & & & & & & & \\
\hline 28 & 105 & 4 & 49.1 & 4 & & & 197.8 & 0 & 594 & 3 & 209 & 1 & 210 & 0 \\
\hline 30 & 106 & 4 & 53.8 & 2 & 410 & 4 & 80.3 & 4 & 629 & 4 & & & & \\
\hline 32 & 108 & 3 & 53.9 & 2 & 390 & 4 & 75.0 & 3 & 630 & 4 & 270 & 0 & 122 & 0 \\
\hline 33 & 104 & 4 & & & & & & & & & 220 & 3 & 092 & 4 \\
\hline 34 & & & & & & & & & & & & & & \\
\hline 35 & & & & & & & & & & & 226 & 4 & & \\
\hline 36 & 86 & 0 & 47.1 & 2 & 446 & 1 & 66.0 & 0 & 600 & 4 & 216 & 2 & 072 & 0 \\
\hline 39 & 9.6 & 2 & 47.1 & 2 & 395 & 4 & 73.0 & 2 & 606 & 4 & 213 & 2 & & \\
\hline 40 & 102 & 4 & 52.0 & 4 & 430 & 2 & 81.0 & 4 & 650 & 3 & 231 & 4 & 0.97 & 4 \\
\hline 42 & 10.6 & 4 & 50.6 & 4 & 400 & 4 & 79.0 & 4 & 624 & 4 & 235 & 3 & 0.89 & 3 \\
\hline 43 & 100 & 3 & & & & & & & & & 230 & 4 & 0,90 & 3 \\
\hline 45 & & & 11.4 & 0 & & & 70.0 & 1 & 60,3 & 4 & & & & \\
\hline 46 & 10.7 & 3 & 45.9 & 2 & 43.0 & 2 & 83.1 & 3 & 654 & 3 & 229 & 4 & 096 & 4 \\
\hline 48 & 110 & 2 & 54.0 & 2 & $<50$ & NR & 87.9 & 1 & 700 & 1 & 200 & 0 & 103 & 3 \\
\hline 50 & & & 53.0 & 3 & 370 & 2 & 80.0 & 4 & 610 & 4 & 196 & 0 & & \\
\hline 52 & 110 & 2 & 50.0 & 4 & 410 & 4 & 79.0 & 4 & 610 & 4 & 220 & 3 & 093 & 4 \\
\hline 54 & 110 & 2 & & & & & & & & & 230 & 4 & 0,90 & 3 \\
\hline 55 & 107 & 3 & 46.4 & 2 & 38.5 & 3 & 81.0 & 4 & 613 & 4 & 223 & 4 & $0: 94$ & 4 \\
\hline 58 & 96 & 2 & 53.0 & 3 & & & 72.0 & 2 & 650 & 3 & 160 & 0 & 097 & 4 \\
\hline 59 & 10.4 & 4 & 52.0 & 4 & 400 & 4 & 81.0 & 4 & 640 & 4 & 226 & 4 & 100 & 4 \\
\hline 60 & & & 54.8 & 2 & & & 85.4 & 2 & 630 & 4 & & & & \\
\hline 64 & 105 & 4 & & & & & & & & & & & 0,90 & 3 \\
\hline 68 & 90 & 0 & 44.0 & 0 & 20.5 & 0 & 70.0 & 1 & 515 & 0 & 200 & 0 & $<015$ & 0 \\
\hline 69 & 104 & 4 & 48.3 & 3 & & & 80.5 & 4 & 590 & 3 & 232 & 4 & 100 & 4 \\
\hline 70 & 111 & 2 & 50.0 & 4 & 40,4 & 4 & 79.6 & 4 & 609 & 4 & 225 & 4 & 100 & 4 \\
\hline 73 & & & 53.5 & 3 & & & 83.9 & 3 & 620 & 4 & 237 & 3 & & 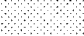 \\
\hline 75 & 101 & 3 & 50.6 & 4 & 40.6 & 4 & 77.3 & 4 & 610 & 4 & 219 & 3 & 0,93 & 4 \\
\hline 76 & & & 52.8 & 3 & & & & & 620 & 4 & 233 & 4 & & \\
\hline 80 & & & 46.0 & 2 & & & & & 620 & 4 & 240 & 2 & & \\
\hline 81 & 95 & 2 & 50.0 & 4 & 370 & 2 & 60.0 & 0 & 600 & 4 & 182 & 0 & 088 & 3 \\
\hline 83 & 102 & 4 & 49.1 & 4 & & & 77.9 & 4 & 621 & 4 & 222 & 3 & 089 & 3 \\
\hline 84 & 100 & 3 & & & & & & & 716 & 0 & & & & \\
\hline 85 & 102 & 4 & 53.1 & 3 & 448 & 1 & 83.8 & 3 & 660 & 3 & 232 & 4 & 1,02 & 3 \\
\hline 86 & 107 & 3 & 49.3 & 4 & 413 & 4 & 65.7 & 0 & 613 & 4 & 232 & 4 & 0.97 & 4 \\
\hline 87 & 300 & 0 & 50.0 & 4 & & & 83.0 & 3 & 600 & 4 & 230 & 4 & 113 & 1 \\
\hline 89 & 101 & 3 & 47.9 & 3 & 465 & 0 & 71.2 & 2 & 576 & 2 & 231 & 4 & 100 & 4 \\
\hline 90 & & & & & & & & & & & 233 & 4 & & \\
\hline 91 & & & & & & & & & & & 227 & 4 & & \\
\hline 92 & 90 & 0 & 50.0 & 4 & 360 & 1 & 70.0 & 1 & 550 & 1 & 215 & 2 & 200 & 0 \\
\hline 96 & & & 46.8 & 2 & & & 78.9 & 4 & 582 & 3 & 220 & 3 & & \\
\hline 97 & 102 & 4 & 53.1 & 3 & 384 & 3 & 70.2 & 1 & 572 & 2 & 217 & 3 & 090 & 3 \\
\hline 100 & 110 & 2 & 51.7 & 4 & 500 & 0 & 77.0 & 4 & 63.3 & 4 & 235 & 3 & 075 & 0 \\
\hline 101 & 107 & 3 & & & & & & & & & & & 1,10 & 1 \\
\hline
\end{tabular}


Table 5. -Laboratory performance ratings for standard reference water sample $T-135$ (trace constituents) --Continued

Analyte $=\mathrm{Ca}(\mathrm{Calcum}), \mathrm{Cd}$ (Cadmium) Co (Cobalt)

F-pseudos

\begin{tabular}{|c|c|c|c|c|c|c|c|c|c|}
\hline & 0.6 & & 3.2 & & 26 & & 5.5 & & \\
\hline Lab & $\mathrm{RV}$ & Rating & RV & Rating & RV & Rating & RV & Rating & \\
\hline 102 & 82 & 0 & 48.0 & 3 & 38,0 & 3 & 73.0 & 2 & 5 \\
\hline 103 & 93 & 1 & 49.0 & 4 & 370 & 2 & 75.0 & 3 & 5 \\
\hline 105 & 10.6 & 4 & 49.0 & 4 & 38.9 & 4 & 74.4 & 3 & 5 \\
\hline 107 & 97 & 2 & & & & & & & 8 \\
\hline 108 & & & & & & & 62.0 & 0 & 8 \\
\hline
\end{tabular}

\begin{tabular}{|c|c|c|c|c|c|c|c|c|c|c|c|c|c|c|}
\hline 108 & & & & & & & 62.0 & 0 & 820 & 0 & & & & \\
\hline 109 & 100 & 3 & & & & & & & & & 227 & 4 & 0.99 & 4 \\
\hline 111 & 123 & 0 & & & & & & & & & & & 108 & 2 \\
\hline 114 & 80 & 0 & 50.0 & 4 & & & 85.0 & 2 & 560 & 2 & 225 & 4 & 100 & 4 \\
\hline 116 & 101 & 3 & & & & & & & & & 245 & 1 & \& 3 & NR \\
\hline 118 & & & 54.0 & 2 & & & 96.5 & 0 & 512 & 0 & 274 & 0 & & \\
\hline 119 & 110 & 2 & 49.3 & 4 & & & 85.5 & 2 & 650 & 3 & 233 & 4 & 100 & 4 \\
\hline 121 & 102 & 4 & 48.0 & 3 & 35.0 & 1 & & & 560 & 2 & 225 & 4 & 100 & 4 \\
\hline 126 & & & 62.0 & 0 & & & 64.0 & 0 & & & & & & \\
\hline 127 & 104 & 4 & 53.1 & 3 & 41.9 & 3 & 81.9 & 3 & 63.4 & 4 & 235 & 3 & 0.97 & 4 \\
\hline 128 & 10.4 & 4 & 51.2 & 4 & 4113 & 4 & 76.0 & 3 & 58.0 & 3 & 206 & 1 & $<0.5$ & 0 \\
\hline 129 & 150 & 0 & & & & & & & & & & & 104 & 3 \\
\hline 132 & 112 & 2 & 47.0 & 2 & 40.0 & 4 & 81.0 & 4 & 64.0 & 4 & 255 & 0 & 118 & 0 \\
\hline 133 & 99 & 3 & 53.3 & 3 & & & 90.0 & 1 & 64.2 & 3 & 261 & 0 & & \\
\hline 134 & 10.8 & 3 & 50.7 & 4 & 40.2 & 4 & 77.8 & 4 & 618 & 4 & 223 & 4 & 0,89 & 3 \\
\hline 138 & $10 \%$ & 3 & 50.6 & 4 & 40.2 & 4 & 78.6 & 4 & 63.6 & 4 & 239 & 3 & 0.90 & 3 \\
\hline 140 & 10.4 & 4 & 50.0 & 4 & & & 85.0 & 2 & 620 & 4 & 210 & 1 & 0.88 & 3 \\
\hline 141 & 10.6 & 4 & 54.0 & 2 & $40: 0$ & 4 & 79.0 & 4 & 65.0 & 3 & 241 & 2 & 093 & 4 \\
\hline 142 & 108 & 3 & 52.6 & 3 & 379 & 3 & 73.9 & 3 & 58.6 & 3 & 238 & 3 & 138 & 0 \\
\hline 145 & 106 & 4 & 52.7 & 3 & 427 & 2 & 80.0 & 4 & 65.2 & 3 & 225 & 4 & 0.91 & 3 \\
\hline 146 & 98 & 2 & 48.7 & 3 & 38.6 & 3 & 77.9 & 4 & 617 & 4 & 224 & 4 & 096 & 4 \\
\hline 149 & & & 44.0 & 1 & & & 76.0 & 3 & & & & & & \\
\hline 151 & 104 & 4 & 50.0 & 4 & & & & & 63.0 & 4 & 230 & 4 & 0.87 & 2 \\
\hline 154 & 10.5 & 4 & 50.0 & 4 & 39.6 & 4 & 71.2 & 2 & 65.4 & 3 & 207 & 1 & 1.52 & 0 \\
\hline 180 & 112 & 2 & 57.2 & 0 & 44.6 & 1 & 87.4 & 1 & 69.2 & 1 & 244 & 2 & 139 & 0 \\
\hline 182 & 10.3 & 4 & 33.6 & 0 & 23.4 & 0 & 89.3 & 1 & 33.4 & 0 & 304 & 0 & 102 & 3 \\
\hline 183 & & & & & & & & & 67.0 & 2 & & & & \\
\hline 190 & 9.0 & 0 & 57.6 & 0 & & & 82.5 & 3 & 67.2 & 2 & 234 & 3 & 0.78 & 1 \\
\hline 191 & 9.4 & 1 & 58.9 & 0 & 40.0 & 4 & 78.1 & 4 & 626 & 4 & 265 & 0 & 0.89 & 3 \\
\hline 193 & 102 & 4 & 47.5 & 3 & 39.0 & 4 & 76.0 & 3 & 54.0 & 1 & 248 & 1 & 0.93 & 4 \\
\hline 194 & 9.5 & 2 & 47.5 & 3 & 41.0 & 4 & 170.0 & 0 & 70.0 & 1 & 240 & 2 & 101 & 3 \\
\hline 196 & 121 & 0 & 50.7 & 4 & 41.1 & 4 & 78.3 & 4 & 65.2 & 3 & & & 094 & 4 \\
\hline 198 & 112 & 2 & 48.7 & 3 & & 3 & 79.6 & 4 & 63.2 & 4 & 233 & 4 & 102 & 3 \\
\hline 203 & 102 & 4 & 43.5 & 0 & & & 80.0 & 4 & 54.5 & 1 & 217 & 3 & 0.95 & 4 \\
\hline 204 & 99 & 3 & & & & & & & 56.6 & 2 & 228 & 4 & 0.87 & 2 \\
\hline 209 & 107 & 3 & & & & & & & & & & & 125 & 0 \\
\hline 210 & 98 & 2 & 52.5 & 3 & 39.5 & 4 & 70.0 & 1 & 600 & 4 & 216 & 2 & 234 & 0 \\
\hline 212 & 116 & 0 & 58.0 & 0 & 46.0 & 0 & 75.0 & 3 & 700 & 1 & 250 & 1 & 100 & 4 \\
\hline 213 & & & 49.5 & 4 & 386 & 3 & 76.9 & 4 & 647 & 3 & 224 & 4 & & \\
\hline 215 & 10.4 & 4 & 52.0 & 4 & 36.0 & 1 & 77.0 & 4 & 64.0 & 4 & 217 & 3 & 106 & 2 \\
\hline 219 & 110 & 2 & & & 56.0 & 0 & 64.0 & 0 & 61.0 & 4 & 300 & 0 & 70000 & 0 \\
\hline 221 & 103 & 4 & 50.5 & 4 & 42.7 & 2 & 82.4 & 3 & 620 & 4 & 221 & 3 & 0,92 & 3 \\
\hline 224 & 10.9 & 3 & 46.3 & 2 & 359 & 1 & & & 570 & 2 & 217 & 3 & 096 & 4 \\
\hline 228 & & & & & & & & & & & & & & \\
\hline 231 & 106 & 4 & 54.8 & 2 & & & 81.3 & 4 & 52.8 & 0 & 197 & 0 & 0.92 & 4 \\
\hline 234 & 10.5 & 4 & 49.6 & 4 & 41.4 & 3 & 77.2 & 4 & 65.1 & 3 & 222 & 3 & 0.95 & 4 \\
\hline 235 & 104 & 4 & 47.0 & 2 & 38.0 & 3 & 75.0 & 3 & 59.0 & 3 & 233 & 4 & 074 & 0 \\
\hline 236 & 10.0 & 3 & 51.0 & 4 & 49.0 & 0 & 78.0 & 4 & 63.0 & 4 & 224 & 4 & 0.98 & 4 \\
\hline 241 & 102 & 4 & 53.0 & 3 & & & 78.7 & 4 & 63.8 & 4 & 221 & 3 & 1.00 & 4 \\
\hline
\end{tabular}


Table 5. -Laboratory performance ratings for standard reference water sample T-135 (trace constituents)

-Continued

(MPV,most probable value; ug/L, micrograms per liter, mg/L. milligrams per liter; Lab, laboratory number; OLR, overall laboratory rating for all reported values;

V/26, number of reported values of 26 possible values; RV, reported value; $<$, less than)

\begin{tabular}{llll|}
\hline Rating & Absolute Z-value & Rating & Absolute Z-value \\
4 (Excellent) & $0.00-0.50$ & 1 (Questionable) & $1.51-2.00$ \\
3 (Good) & $0.51-1.00$ & $O$ (Poor) & greater than 2.00 \\
2 (Satisfactory) & $1.01-1.50$ & NR (Not Rated) & \\
\hline
\end{tabular}

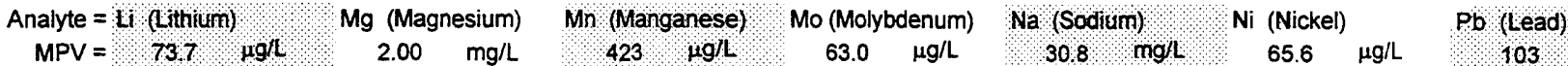

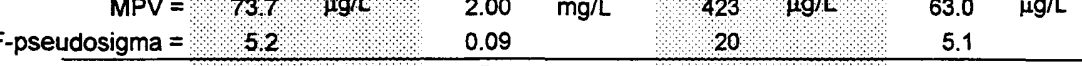

\begin{tabular}{|c|c|c|c|c|c|c|c|c|c|c|c|c|c|c|}
\hline Lab & $\mathrm{RV}$ & Rating & RV & Rating & RV & Rating & $\mathrm{RV}$ & Rating & RV & Rating & RV & Rating & $\mathrm{RV}$ & Rating \\
\hline 1 & 73.6 & मै & 2.02 & 4 & 432 & स 4 - & 66.7 & 3 & 304 & मे & 65.1 & 4 & 100 & 3ै \\
\hline 3 & 770 & 3 & 2.00 & 4 & 405 & 3 & 54.0 & 1 & 313 & 4 & 67.0 & 4 & 105 & 4 \\
\hline 4 & 1420 & 0 & 4.23 & 0 & 825 & 0 & & & 639 & 0 & 119.0 & 0 & 136 & 0 \\
\hline 7 & $\begin{array}{l}723 \\
\end{array}$ & 4 & 2.00 & 4 & 418 & 4 & 61.5 & 4 & 30,9 & 4 & 62.3 & 3 & 94 & 2 \\
\hline 10 & & & & & 425 & 4 & & & & & & & & \\
\hline 11 & & & 1.82 & 1 & 470 & 0 & 70.0 & 2 & 326 & 1 & 70.0 & 3 & 120 & 0 \\
\hline 13 & & & 2.10 & 2 & 440 & 3 & & & 334 & 0 & 58.0 & 1 & 121 & 0 \\
\hline 15 & 781 & 3 & 2.12 & 2 & 440 & 3 & 53.4 & 1 & 30.9 & 4 & 67.9 & 4 & 104 & 4 \\
\hline 16 & 770 & 3 & 2.00 & 4 & 398 & 2 & 62.0 & 4 & 29.7 & 3 & 64.0 & 4 & 81 & 0 \\
\hline 18 & & & 2.00 & 4 & 427 & 4 & 63.0 & 4 & 310 & 4 & 66.0 & 4 & 111 & 2 \\
\hline 19 & & & 2.28 & 0 & 454 & 1 & & & 314 & 3 & & & 100 & 4 \\
\hline 21 & & & & & & & & & & & & & & \\
\hline 23 & & & & & 390 & 1 & & & 32.4 & 2 & & & 105 & 4 \\
\hline 24 & 740 & 4 & 2.00 & 4 & 435 & 3 & 65.0 & 4 & 301 & 3 & 66.2 & 4 & 99 & 3 \\
\hline 25 & 670 & 2 & 1.92 & 3 & 384 & 1 & & & 297 & 3 & $<49$ & 0 & 187 & 0 \\
\hline 26 & 740 & 4 & 2.07 & 3 & 440 & 3 & & & 311 & 4 & 66.7 & 4 & 108 & 3 \\
\hline 27 & & & 2.24 & 0 & & & & & 317 & 3 & & & & \\
\hline 28 & 865 & 0 & 2.10 & 2 & 391 & 1 & 65.5 & 4 & 283 & 0 & 69.0 & 3 & 153 & 0 \\
\hline 30 & 696 & 3 & 1.94 & 3 & 423 & 4 & 64.4 & 4 & & & 66.9 & 4 & 101 & 4 \\
\hline 32 & 790 & 2 & 2.18 & 1 & 409 & 3 & 67.6 & 3 & 319 & 3 & 64.6 & 4 & 99 & 3 \\
\hline 33 & & & 2.04 & 4 & 220 & 0 & & & 309 & 4 & & & & \\
\hline 34 & & & & & & & & & & & & & & \\
\hline 35 & & & & & & & & & & & & & & \\
\hline 36 & & & 2.06 & 3 & 350 & 0 & 27.3 & 0 & 302 & 3 & 47.1 & 0 & 85 & 0 \\
\hline 39 & 707 & 3 & 1.97 & 4 & 386 & 1 & 63.1 & 4 & 29.9 & 3 & 71.7 & 2 & 132 & 0 \\
\hline 40 & 75.0 & 4 & 2.00 & 4 & 435 & 3 & 64.0 & 4 & 306 & 4 & 67.0 & 4 & 117 & 0 \\
\hline 42 & 732 & 4 & 2.10 & 2 & 424 & 4 & 64.0 & 4 & 314 & 3 & 62.8 & 3 & 106 & 4 \\
\hline 43 & & & 2.00 & 4 & 430 & 4 & & & 310 & 4 & & & & \\
\hline 45 & & & & & & & & & & & & & & \\
\hline 46 & & & 2.09 & 2 & 420 & 4 & & & 31.6 & 3 & 73.6 & 1 & 100 & 3 \\
\hline 48 & & & 2.05 & 3 & 440 & 3 & 61.5 & 4 & 298 & 3 & 65.2 & 4 & 140 & 0 \\
\hline 50 & & & & & 428 & 4 & 51.0 & 0 & & & 71.0 & 2 & 106 & 4 \\
\hline 52 & & & 2.00 & 4 & 420 & 4 & 62.0 & 4 & 310 & 4 & 65.0 & 4 & 100 & 4 \\
\hline 54 & & & 2.00 & 4 & 430 & 4 & & & 310 & 4 & & & & \\
\hline 55 & 660 & 2 & 2.19 & 0 & 431 & 4 & 52.5 & 0 & 301 & 3 & 62.3 & 3 & 109 & 3 \\
\hline 58 & & & 25.00 & 0 & 440 & 3 & & & 330 & 1 & 69.0 & 3 & 97 & 3 \\
\hline 59 & & & 2.20 & 0 & 425 & 4 & 70.0 & 2 & 350 & 0 & 67.0 & 4 & 107 & 3 \\
\hline 60 & & & & & & & 65.7 & 3 & & & 77.0 & 0 & 100 & 4 \\
\hline 64 & 800 & 2 & 1.95 & 3 & & & & & 307 & 4 & & & & \\
\hline 68 & 650 & 1 & 1.05 & 0 & $\begin{array}{r}36 \\
\end{array}$ & 0 & 69.5 & 2 & 28.0 & 0 & 55.0 & 0 & 104 & 4 \\
\hline 69 & 805 & 2 & 2.10 & 2 & 426 & 4 & & & 300 & 3 & 64.5 & 4 & 109 & 3 \\
\hline 70 & & & 2.13 & 2 & 415 & 4 & 56.8 & 2 & 317 & 3 & 69.1 & 3 & 100 & 4 \\
\hline 73 & & & & & & & & & & & 67.2 & 4 & 115 & 1 \\
\hline 75 & 703 & 3 & 2.00 & 4 & 414 & 4 & 61.3 & 4 & 310 & 4 & 65.1 & 4 & 103 & 4 \\
\hline 76 & & & & & 435 & 3 & & & & & 63.1 & 4 & 103 & 4 \\
\hline 80 & & & & & 452 & 2 & & & & & & & 103 & 4 \\
\hline 81 & & & 1.95 & 3 & 399 & 2 & 59.0 & 3 & 297 & 3 & 40.0 & 0 & 104 & 4 \\
\hline 83 & & & 1.99 & 4 & 412 & 3 & & & 307 & 4 & 69.5 & 3 & 102 & 4 \\
\hline 84 & & & 2.00 & 4 & & & & & 30.6 & 4 & & & 106 & 3 \\
\hline 85 & 751 & 4 & 1.98 & 4 & 422 & 4 & 70.0 & 2 & 30.2 & 3 & 66.0 & 4 & 117 & 0 \\
\hline 86 & & & 2.07 & 3 & 426 & 4 & 61.2 & 4 & 314 & 3 & 65.2 & 4 & 99 & 3 \\
\hline 87 & & & 1.90 & 2 & 408 & 3 & 58.6 & 3 & 10,0 & 0 & 73.0 & 2 & 126 & 0 \\
\hline 89 & & & 2.00 & 4 & 424 & 4 & & & 289 & 1 & 61.9 & 3 & 105 & 4 \\
\hline 90 & & & & & 352 & 0 & & & & & & & & \\
\hline 91 & & & & & 435 & 3 & & & & & & & & \\
\hline 92 & & & 2.00 & 4 & 42 & 0 & & & 200 & 0 & & & 100 & 4 \\
\hline 96 & & & & & 440 & 3 & & & & & & & 99 & 3 \\
\hline 97 & & & 2.00 & 4 & 408 & 3 & 58.2 & 3 & 312 & 4 & 61.3 & 3 & 99 & 3 \\
\hline 100 & 80,3 & 2 & 2.10 & 2 & 437 & 3 & 54.3 & 1 & 340 & 0 & 66.7 & 4 & 113 & 1 \\
\hline 101 & & & 2.00 & 4 & & & & & 315 & 3 & & & & \\
\hline
\end{tabular}


Table 5. -Laboratory performance ratings for standard reference water sample T-135 (trace constituents) --Continued

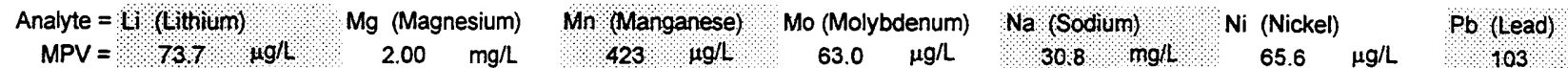

\begin{tabular}{|c|c|c|c|c|c|c|c|c|c|c|c|c|c|c|}
\hline $\begin{array}{r}\text { MPV }= \\
\text { F-pseudosigma }=\end{array}$ & 52 & $\lg$ & $\begin{array}{l}2.00 \\
0.09\end{array}$ & $\mathrm{mg} / \mathrm{L}$ & 223 & HLL & $\begin{array}{r}63.0 \\
5.1\end{array}$ & $\mu g / L$ & 308 & righ & $\begin{array}{r}65.6 \\
5.0\end{array}$ & $\mu g / L$ & 103 & $\mu \mathrm{g} / \mathrm{L}$ \\
\hline Lab & RV & Rating & $\mathrm{RV}$ & Rating & $\mathrm{RV}$ & Rating & RV & Rating & RV & Rating & RV & Rating & $\mathrm{RV}$ & Rating \\
\hline 102 & & & 1.80 & 0 & 405 & 3 & & & 182 & 0 & 62.0 & 3 & 95 & 2 \\
\hline 103 & 690 & 3 & 1.90 & 2 & 426 & 4 & 63.0 & 4 & 300 & 3 & 54.0 & 0 & 830 & 0 \\
\hline 105 & 790 & 2 & 2.05 & 3 & 424 & 4 & 66.9 & 3 & 316 & 3 & 60.1 & 2 & 104 & 4 \\
\hline 107 & & & 2.01 & 4 & 430 & 4 & & & 29.4 & 2 & & & 100 & 4 \\
\hline 108 & & & & & & & 57.9 & 3 & & & 55.0 & 0 & & \\
\hline 109 & 573 & 0 & 2.00 & 4 & 417 & 4 & 74.4 & 0 & 285 & 1 & & & 74 & 0 \\
\hline 111 & & & 2.04 & 4 & & & & & 30.6 & 4 & & & & \\
\hline 114 & & & 2.00 & 4 & 420 & 4 & & & 170 & 0 & 67.0 & 4 & 160 & 0 \\
\hline 116 & $<80$ & NR & 1.96 & 4 & 425 & 4 & & & 314 & 3 & & & & \\
\hline 118 & & & & & 337 & 0 & & & & & 73.4 & 1 & 105 & 4 \\
\hline 119 & & & 2.30 & 0 & 442 & 3 & 55.0 & 1 & 328 & 1 & 68.0 & 4 & 106 & 4 \\
\hline 121 & & & 2.03 & 4 & 410 & 3 & & & 310 & 4 & 62.0 & 3 & & \\
\hline 126 & & & & & & & & & & & 75.0 & 1 & 98 & 3 \\
\hline 127 & 669 & 2 & 2.07 & 3 & 417 & 4 & 61.6 & 4 & 296 & 2 & 63.3 & 4 & 97 & 3 \\
\hline 128 & & & 1.85 & 1 & 407 & 3 & 56.9 & 2 & 262 & 0 & 55.3 & 0 & 100 & 4 \\
\hline 129 & & & 0.31 & 0 & 420 & 4 & & & 300 & 3 & & & & \\
\hline 132 & & & 2.06 & 3 & 403 & 3 & 69.0 & 2 & 318 & 3 & 70.0 & 3 & 96 & 2 \\
\hline 133 & & & 1.93 & 3 & & & & & & & 68.0 & 4 & 111 & 2 \\
\hline 134 & 790 & 2 & 2.05 & 3 & 430 & 4 & 60.8 & 4 & 30,3 & 4 & 68.2 & 3 & 105 & 4 \\
\hline 138 & & & 2.09 & 2 & 416 & 4 & 67.7 & 3 & 318 & 3 & 64.2 & 4 & 99 & 3 \\
\hline 140 & & & 2.00 & 4 & 430 & 4 & & & 380 & 0 & 62.0 & 3 & 100 & 4 \\
\hline 141 & & & 2.13 & 2 & 428 & 4 & 66.0 & 3 & 300 & 3 & 67.0 & 4 & 88 & 0 \\
\hline 142 & 737 & 4 & 2.03 & 4 & 425 & 4 & 66.7 & 3 & 298 & 3 & 59.8 & 2 & 106 & 4 \\
\hline 145 & 711 & 4 & 2.02 & 4 & 425 & 4 & 62.5 & 4 & 302 & 3 & 68.0 & 4 & 113 & 2 \\
\hline 146 & & & 1.96 & 4 & 418 & 4 & 61.5 & 4 & 30.8 & 4 & 64.3 & 4 & 101 & 4 \\
\hline 149 & & & & & & & & & & & & & 102 & 4 \\
\hline 151 & 710 & 3 & 2.00 & 4 & 435 & 3 & & & 315 & 3 & & & & \\
\hline 154 & & & 1.80 & 0 & 396 & 2 & 61.5 & 4 & 30.5 & 4 & 61.0 & 3 & 104 & 4 \\
\hline 180 & & & 2.20 & 0 & 462 & 1 & 67.6 & 3 & 33.2 & 1 & 73.7 & 1 & 112 & 2 \\
\hline 182 & 650 & 1 & 1.98 & 4 & 475 & 0 & 74.8 & 0 & 239 & 0 & 78.8 & 0 & 89 & 0 \\
\hline & \multicolumn{14}{|c|}{183} \\
\hline 190 & & & 2.29 & 0 & 416 & 4 & & & $31 / 4$ & 3 & 64.1 & 4 & 95 & 2 \\
\hline 191 & & & 1.99 & 4 & 417 & 4 & & & 30.1 & 3 & 66.2 & 4 & & \\
\hline 193 & & & 1.98 & 4 & & & & & 30.8 & 4 & & & 113 & 1 \\
\hline 194 & & & 1.88 & 2 & 450 & 2 & 80.0 & 0 & 31,3 & 4 & $<100$ & NR & 106 & 4 \\
\hline 196 & 715 & 4 & 1.90 & 2 & 469 & 0 & 63.4 & 4 & 319 & 3 & 64.9 & 4 & 107 & 3 \\
\hline 198 & & & 2.16 & 1 & 456 & 1 & 62.0 & 4 & 29.3 & 2 & 69.6 & 3 & 108 & 3 \\
\hline 203 & & & 2.02 & 4 & 360 & 0 & & & 30.3 & 4 & 57.0 & 1 & 87 & 0 \\
\hline 204 & & & 2.01 & 4 & 417 & 4 & & & 30.2 & 3 & & & & \\
\hline 209 & & & 2.11 & 2 & & & & & 29.1 & 2 & & & & \\
\hline 210 & & & 1.76 & 0 & 406 & 3 & 65.0 & 4 & 276 & 0 & 70.5 & 3 & 100 & 4 \\
\hline 212 & 770 & 3 & 2.20 & 0 & 480 & 0 & 71.0 & 1 & 353 & 0 & 77.0 & 0 & 93 & 1 \\
\hline 213 & & & & & & & & & & & 69.0 & 3 & 99 & 3 \\
\hline 215 & & & 1.98 & 4 & 406 & 3 & 60.0 & 3 & 296 & 2 & 65.0 & 4 & 104 & 4 \\
\hline 219 & 720 & 4 & 1.90 & 2 & 400 & 2 & & & 260 & 0 & 68.0 & 4 & & \\
\hline 221 & & & 1.95 & 3 & 440 & 3 & 70.0 & 2 & 312 & 4 & 70.3 & 3 & 108 & 3 \\
\hline 224 & & & 2.09 & 2 & 396 & 2 & 22.0 & 0 & 30.9 & 4 & 59.1 & 2 & 94 & 2 \\
\hline & \multicolumn{14}{|c|}{228} \\
\hline 231 & & & 2.05 & 3 & 408 & 3 & & & 313 & 4 & 53.0 & 0 & 108 & 3 \\
\hline 234 & 721 & 4 & 1.95 & 3 & 404 & 3 & 60.9 & 4 & 30.8 & 4 & 64.4 & 4 & 96 & 2 \\
\hline 235 & & & 1.97 & 4 & 430 & 4 & 70.0 & 2 & & & 64.0 & 4 & 97 & 3 \\
\hline 236 & 740 & 4 & 1.98 & 4 & 417 & 4 & 66.0 & 3 & 320 & 2 & 63.0 & 3 & 99 & 3 \\
\hline 241 & & $\cdots$ & 1.90 & 2 & 479 & 4 & & & 275 & 0 & 34.2 & 0 & 90 & 1 \\
\hline
\end{tabular}


Table 5. -Laboratory performance ratings for standard reference water sample T-135 (trace constituents)

\begin{tabular}{|c|c|c|c|c|c|c|c|c|c|c|c|c|}
\hline & \multicolumn{12}{|c|}{$\begin{array}{l}\text { (MPV,most probable value; ug/L, micrograms per liter, } m g / L \text {, milligrams per liter, Lab, laboratory number, } O L R \text {, overall laboratory rating for all reportec } \\
\text { VI26, number of reported values of } 26 \text { possible values; } R V \text {, reported value; }<\text {, less than) }\end{array}$} \\
\hline & & \multicolumn{2}{|c|}{ Absolute Z-value } & \multicolumn{2}{|c|}{ Rating } & \multicolumn{2}{|c|}{ Absolute Z-value } & & & & \\
\hline & \multicolumn{2}{|c|}{4 (Excellent) } & \multicolumn{2}{|c|}{$0.00-0.50$} & \multicolumn{2}{|c|}{1 (Questionable) } & $1.51-2.00$ & & & & & \\
\hline & 3 (Good) & & $0.51-1.00$ & & $O$ (Poor) & & greater the & 2.00 & & & & \\
\hline & & & $1.01-1.50$ & & $N R$ (Not $R$ & & & & & & & \\
\hline Analyte $=$ & Sb (Antir & ony) & Se (Sele & um) & $\mathrm{SiO}_{2}$ (Si) & & Sr (Stror & ium) & Voana & (m) & Zn (Zinc) & \\
\hline$M P V=$ & $\begin{array}{r}163 \\
\end{array}$ & $\mu \mathrm{g} / \mathrm{L}$ & 10.0 & $\mu \mathrm{g} / \mathrm{L}$ & 428 & $\mathrm{mg} / \mathrm{L}$ & 46.0 & $\mu g / L$ & 52.8 & $\mu g /$ & 48.2 & $\mu \mathrm{g} / \mathrm{L}$ \\
\hline F-pseudosigma = & 87 & & 1.4 & & 0.31 & & 2.3 & & 36 & & 4.7 & \\
\hline Lab & RV & Rating & RV & Rating & $\mathrm{RV}$ & Rating & $\mathrm{RV}$ & Rating & $\mathrm{RV}$ & Rating & RV & Rating \\
\hline 1 & 823 & 3 & 10.1 & 4 & 4.29 & 4 3 & 47.1 & 4 & 529 & 4 & 65.3 & 0 \\
\hline 3 & 780 & 4 & 9.4 & 4 & 4.46 & 3 & 44.3 & 3 & 54,0 & 4 & 48.2 & 4 \\
\hline 4 & & & & & 950 & 0 & 98.7 & 0 & & & 85.0 & 0 \\
\hline 7 & 69.7 & 3 & 25.0 & 0 & 4.66 & 2 & 46.5 & 4 & 54.4 & 4 & 48.2 & 4 \\
\hline 10 & & & & & & & & & & & 51.0 & 3 \\
\hline 11 & 700 & 3 & & & 4.62 & 2 & & & 500 & 3 & 40.0 & 1 \\
\hline 13 & 74.5 & 4 & 7.6 & 1 & 440 & 4 & & & 570 & 2 & 52.0 & 3 \\
\hline 15 & 672 & 2 & 8.9 & 3 & 415 & 4 & 44.2 & 3 & 56.6 & 2 & 52.7 & 3 \\
\hline 16 & 63.0 & 1 & $<70$ & NR & & & 46.0 & 4 & 510 & 4 & 51.0 & 3 \\
\hline 18 & 76.0 & 4 & 11.6 & 2 & & & 46.0 & 4 & 540 & 4 & 87.0 & 0 \\
\hline 19 & & & & & & & & & & & 66.0 & 0 \\
\hline 21 & & & & & & & & & & & & \\
\hline 23 & & & & & 301 & 0 & 43.6 & 2 & & & & \\
\hline 24 & 82.8 & 3 & 42.6 & 0 & 428 & 4 & 48.6 & 2 & 581 & 2 & 76.5 & 0 \\
\hline 25 & 89.0 & 2 & 9.2 & 3 & 492 & 0 & 42.0 & 1 & 430 & 0 & $<4$ & 0 \\
\hline 26 & & & 6.0 & 0 & & & & & & & 46.1 & 4 \\
\hline 27 & & & & & & & & & & & & \\
\hline 28 & & & 11.0 & 3 & 3,50 & 0 & 45.5 & 4 & 554 & 3 & 35.2 & 0 \\
\hline 30 & 828 & 3 & 12.2 & 1 & & & & & 55.6 & 3 & 49.4 & 4 \\
\hline 32 & 79.6 & 4 & & & & & 46.6 & 4 & 569 & 2 & 46.5 & 4 \\
\hline 33 & & & & & 4.07 & 3 & 50.0 & 1 & & & & \\
\hline 34 & & & 10.1 & 4 & & & & & & & & \\
\hline 35 & & & 9.3 & 4 & & & & & & & & \\
\hline 36 & 68,6 & 3 & 9.1 & 3 & & & & & 476 & 2 & 60.5 & 0 \\
\hline 39 & 812 & 3 & 11.0 & 3 & 194 & 0 & 44.1 & 3 & 475 & 2 & 48.5 & 4 \\
\hline 40 & 810 & 3 & & & & & 46.0 & 4 & 540 & 4 & 57.0 & 1 \\
\hline 42 & 815 & 3 & 11.0 & 3 & 4.30 & 4 & 48.0 & 3 & 53.0 & 4 & 49.2 & 4 \\
\hline 43 & & & & & 4220 & 4 & & & & & & \\
\hline 45 & & & 12.4 & 1 & & & & & & & & \\
\hline 46 & & & 22.7 & 0 & & & & & 541 & 4 & 48.2 & 4 \\
\hline 48 & 68.8 & 3 & 9.0 & 3 & & & & & 517 & 4 & 60.0 & 0 \\
\hline 50 & & & 10.0 & 4 & & & & & 370 & 0 & 46.0 & 4 \\
\hline $\begin{array}{l}52 \\
54\end{array}$ & 650 & 2 & $<5$ & 0 & 4.21 & 4 & 46.0 & 4 & 490 & 2 & 52.0 & 3 \\
\hline 55 & 747 & 4 & 9.9 & 4 & 3.84 & 2 & & & 530 & 4 & 48.0 & 4 \\
\hline 58 & & & 3.0 & 0 & & & & & & & 71.0 & 0 \\
\hline 59 & 84.0 & 3 & 10.0 & 4 & & & 48.0 & 3 & 470 & 1 & 45.0 & 3 \\
\hline 60 & & & 11.2 & 3 & & & & & & & 50.0 & 4 \\
\hline 64 & & & & & 3.90 & 2 & & & & & & \\
\hline 68 & 73.0 & 4 & 9.3 & 4 & & & 41.5 & 1 & 315 & 0 & 41.0 & 1 \\
\hline 69 & 712 & 3 & 10.6 & 4 & & & & & & & 46.0 & 4 \\
\hline 70 & 65.4 & 2 & 11.0 & 3 & 4.00 & 3 & 47.0 & 4 & 53,3 & 4 & 82.4 & 0 \\
\hline 73 & & & & & & & & & & & 51.1 & 3 \\
\hline 75 & 82.8 & 3 & 9.6 & 4 & & & & & 527 & 4 & 52.8 & 3 \\
\hline 76 & 75.2 & 4 & & & & & & & & & 63.9 & 0 \\
\hline 80 & & & 12.0 & 2 & & & & & & & 49.0 & 4 \\
\hline 81 & 89.0 & 2 & 10.0 & 4 & & & 44.0 & 3 & 510 & 4 & 43.0 & 2 \\
\hline 83 & & & & & 3.97 & 3 & & & & & 44.9 & 3 \\
\hline 84 & & & & & & & & & & & & \\
\hline 85 & 960 & 0 & 9.5 & 4 & & & 46.6 & 4 & 49,1 & 2 & 47.9 & 4 \\
\hline 86 & & & 9.0 & 3 & & & 46.4 & 4 & 53.5 & 4 & 47.6 & 4 \\
\hline 87 & & & $<2$ & 0 & 4.36 & 4 & & & \% & & 49.0 & 4 \\
\hline 89 & 69.6 & 3 & $<2$ & 0 & 4,00 & 3 & & & 458 & 1 & 36.9 & 0 \\
\hline 90 & & & & & & & & & & & 45.0 & 3 \\
\hline 91 & & & & & & & & & & & & \\
\hline 92 & & & & & 447 & 3 & & & & & 110.0 & 0 \\
\hline 96 & & & 9.7 & 4 & & & & & & & 46.0 & 4 \\
\hline 97 & 754 & 4 & 18.6 & 0 & 4.32 & 4 & 43.5 & 2 & 474 & 2 & 43.7 & 3 \\
\hline 100 & 790 & 4 & 7.8 & 1 & 403 & 3 & 37.8 & 0 & 518 & 4 & 52.0 & 3 \\
\hline
\end{tabular}


Table 5. -Laboratory performance ratings for standard reference water sample $T$-135 (trace constituents) --Continued

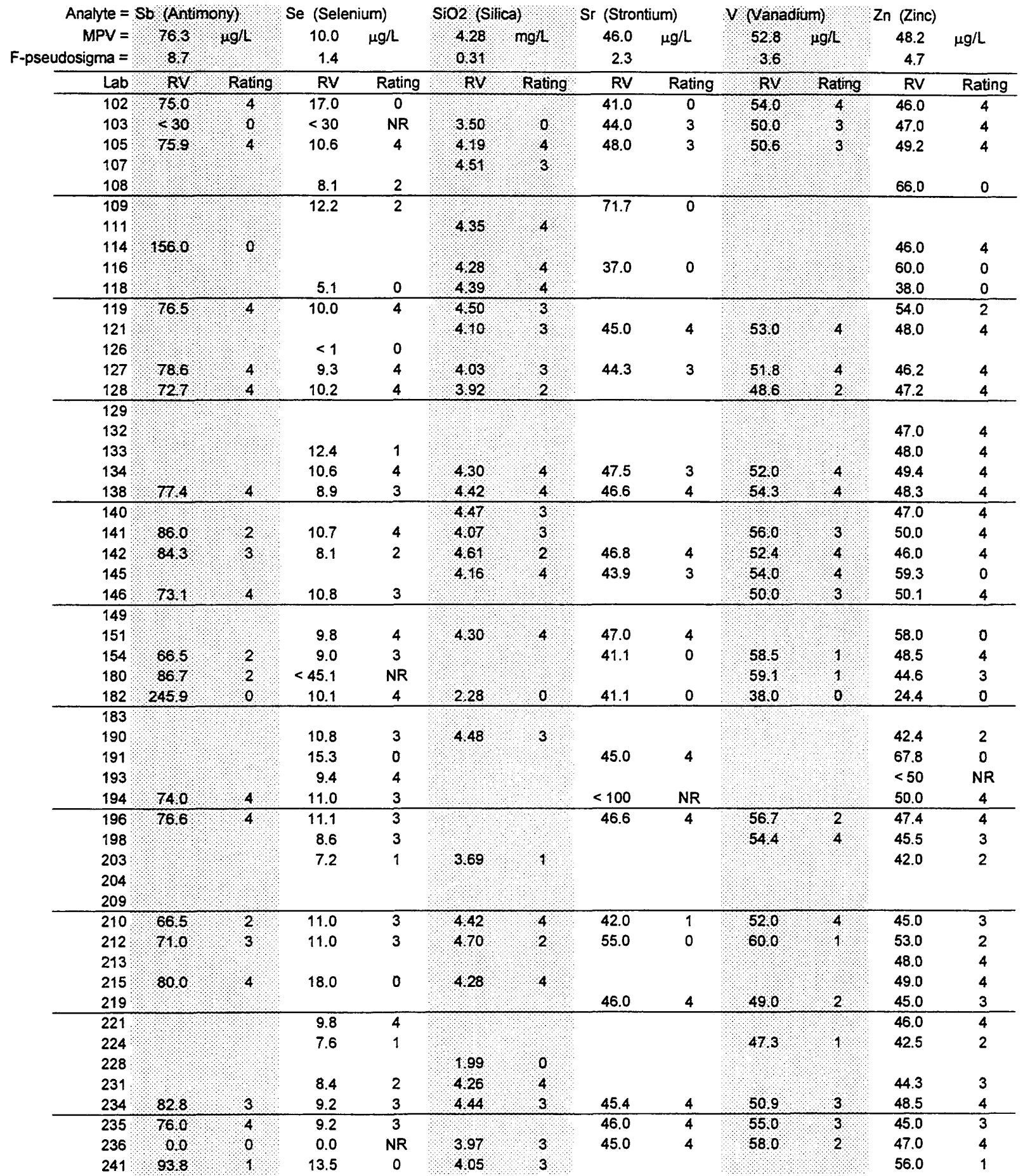


Table 6. -Laboratory performance ratings for standard reference water sample M-134 (major constituents)

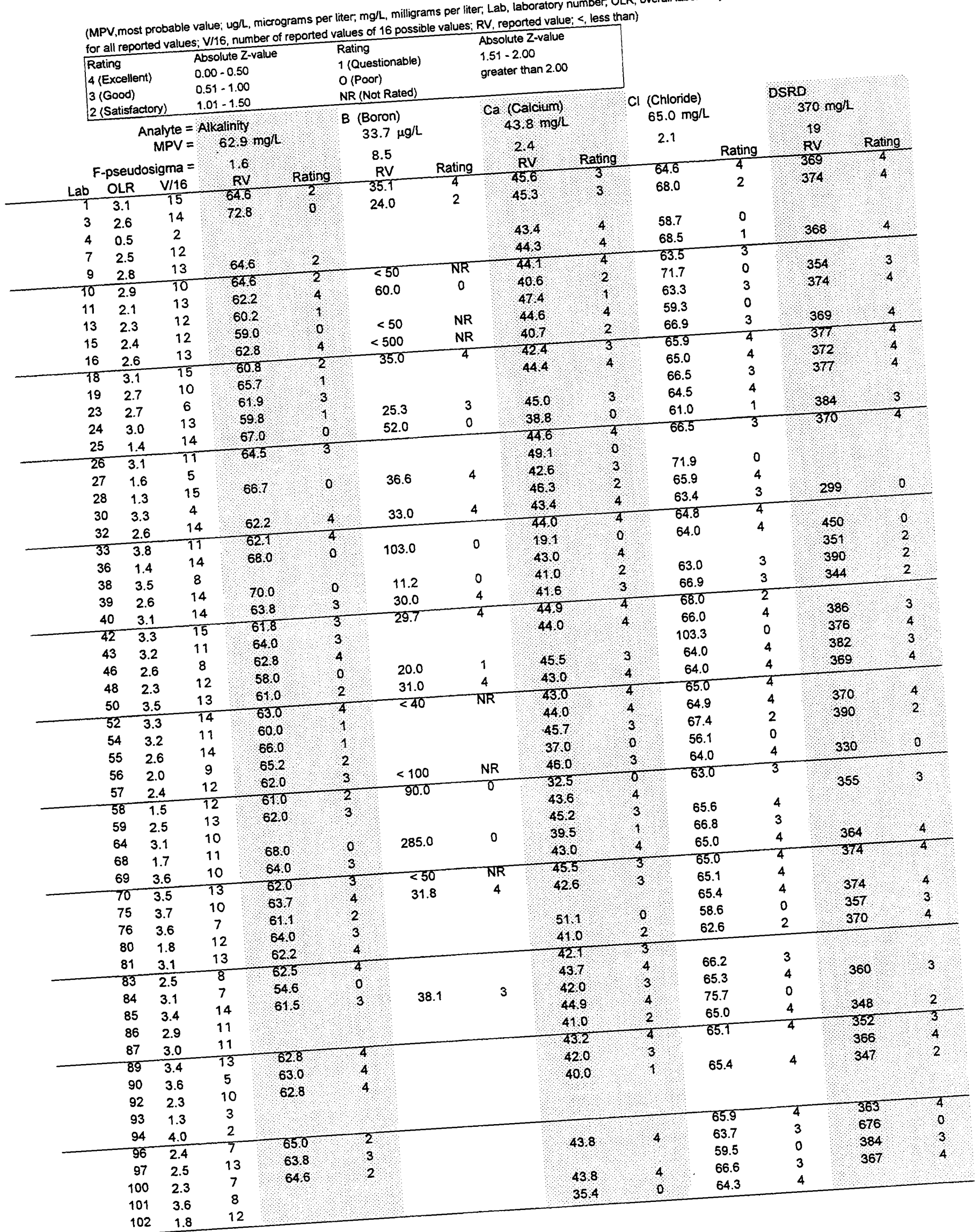


Table 6. -Laboratory performance ratings for standard reference water sample $M-134$ (major constituents) --Continued

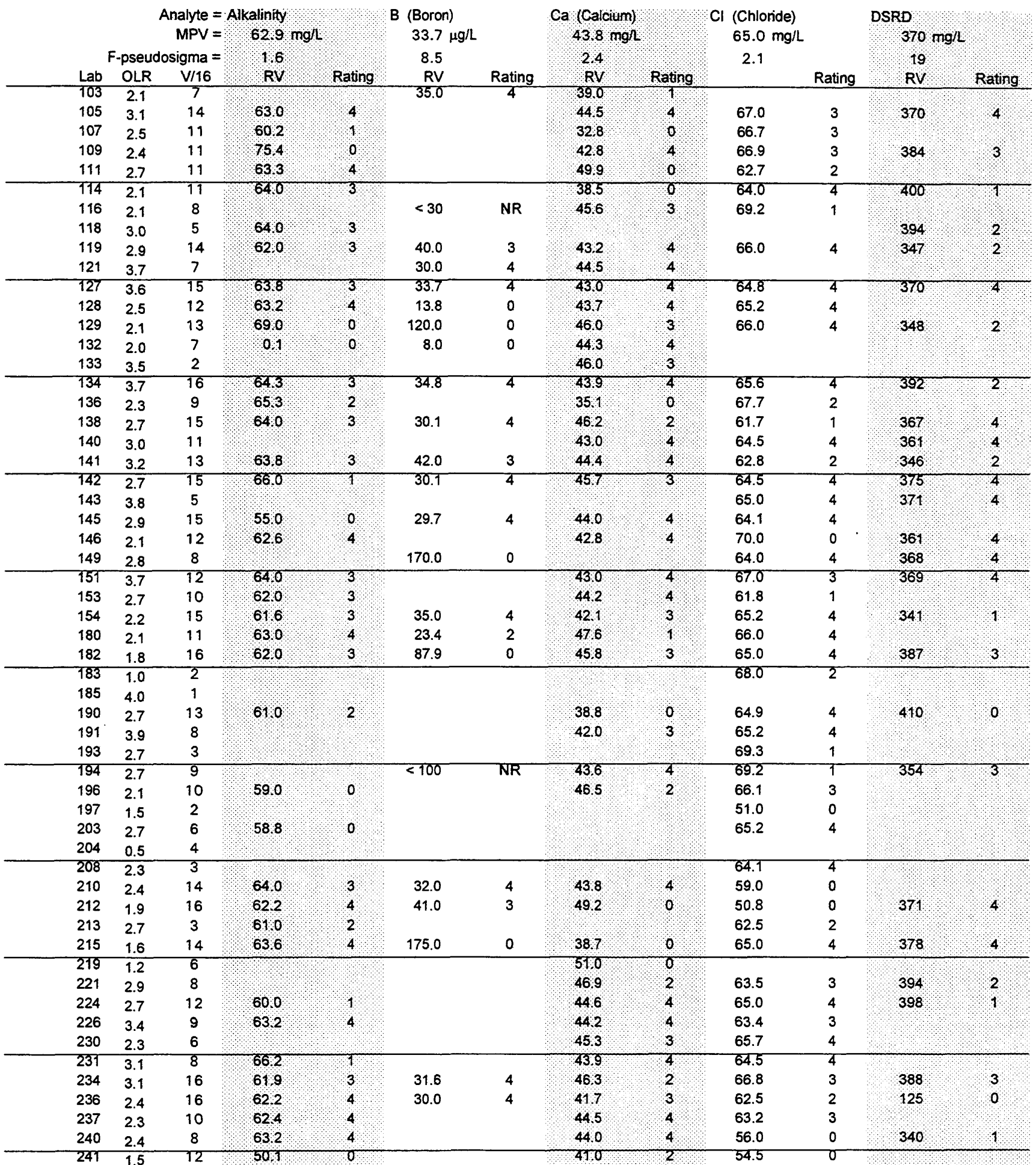


Table 6. -Laboratory performance ratings for standard reference water sample $M-134$ (major constituents) --Continued

(MPV,most probable value; ug/L, micrograms per liter, $\mathrm{mg} / \mathrm{L}$, milligrams per liter, Lab, laboratory number, OLR, overall laboratory rating for all reported values; V/16, number of reported values of 16 possible values; RV, reported value; < less than)

\begin{tabular}{|llll|}
\hline Rating & Absolute Z-value & Rating & Absolute Z-value \\
4 (Excellent) & $0.00-0.50$ & 1 (Questionable) & $1.51-2.00$ \\
3 (Good) & $0.51-1.00$ & $O$ (Poor) & greater than 2.00 \\
2 (Satisfactory) & $1.01-1.50$ & NR (Not Rated) & \\
\hline
\end{tabular}

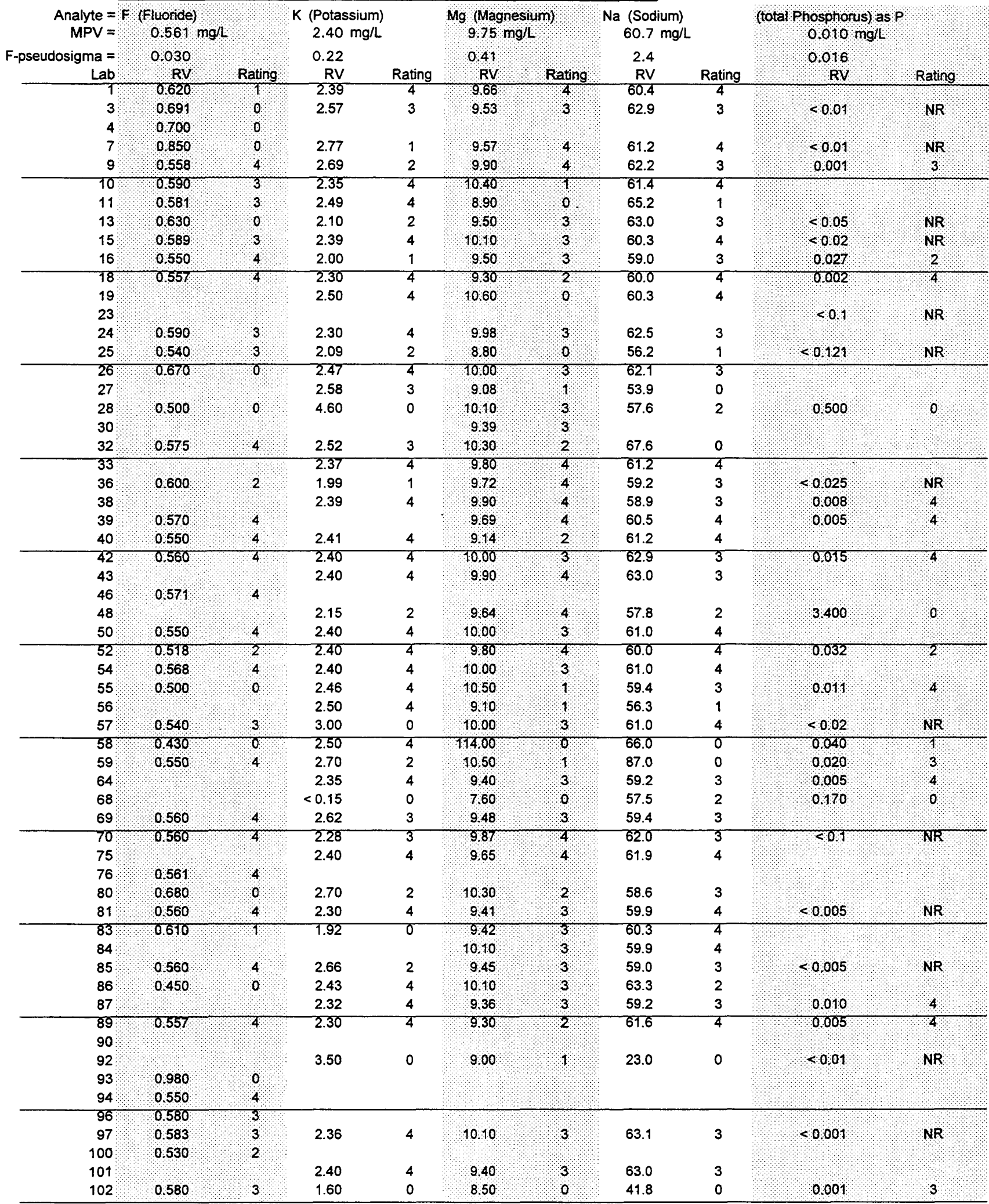


Table 6. -Laboratory performance ratings for standard reference water sample M-134 (major constituents) --Continued

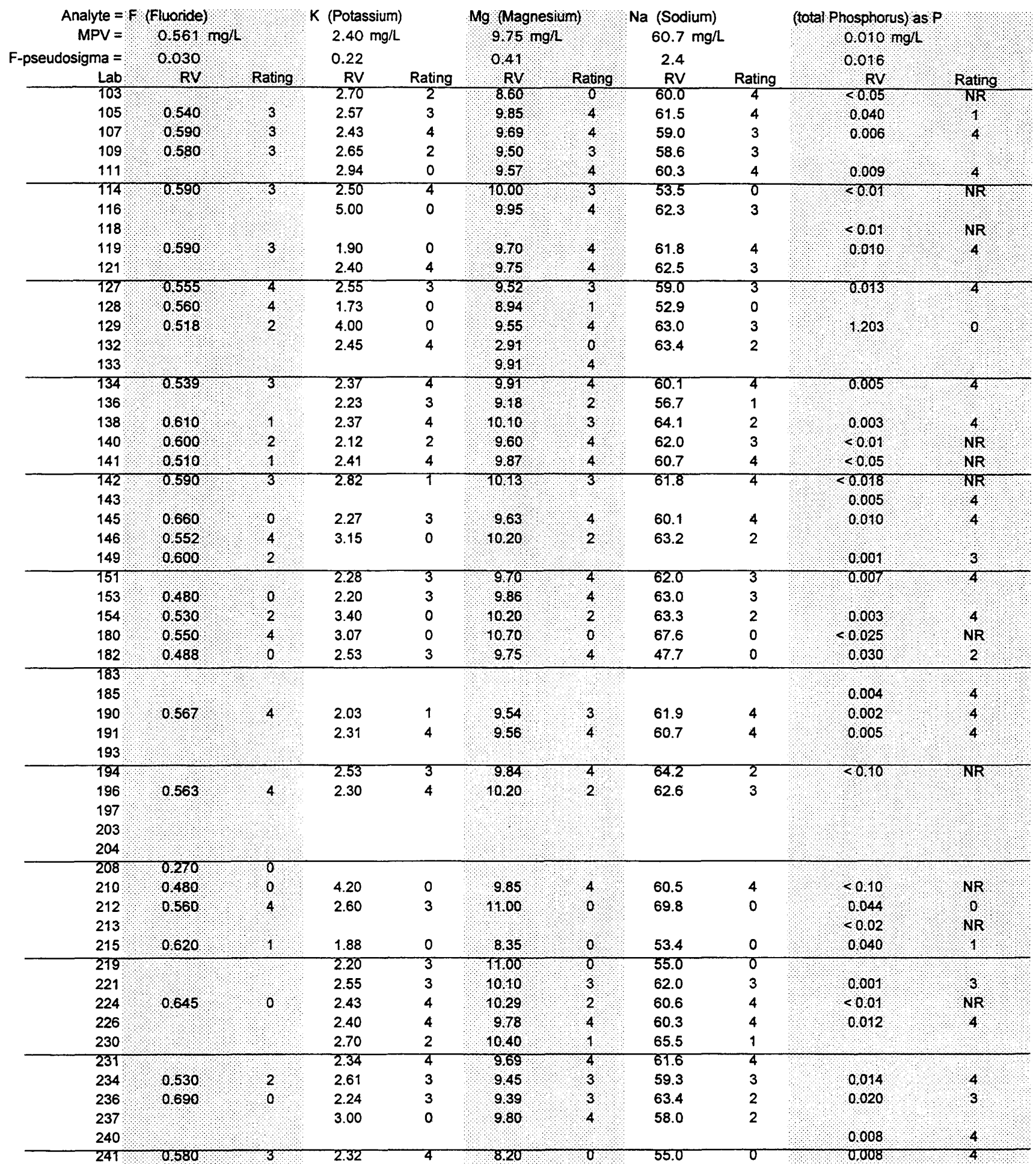


Table 6. -Laboratory performance ratings for standard reference water sample $M-134$ (major constituents) --Continued

(MPV,most probable value; ugh, micrograms per liter, mgl, milligrams per liter, Lab, laboratory number, OLR, overall laboratory rating for all reported values; V/16, number of reported values of 16 possible values; $R V$, reported value; $<$, less than)

\begin{tabular}{|llll|}
\hline Rating & Absolute Z-value & Rating & Absolute Z-value \\
4 (Excellent) & $0.00-0.50$ & 1 (Questionable) & $1.51-2.00$ \\
3 (Good) & $0.51-1.00$ & O (Poor) & greater than 2.00 \\
2 (Satisfactory) & $1.01-1.50$ & NR (Not Rated) & \\
\hline
\end{tabular}

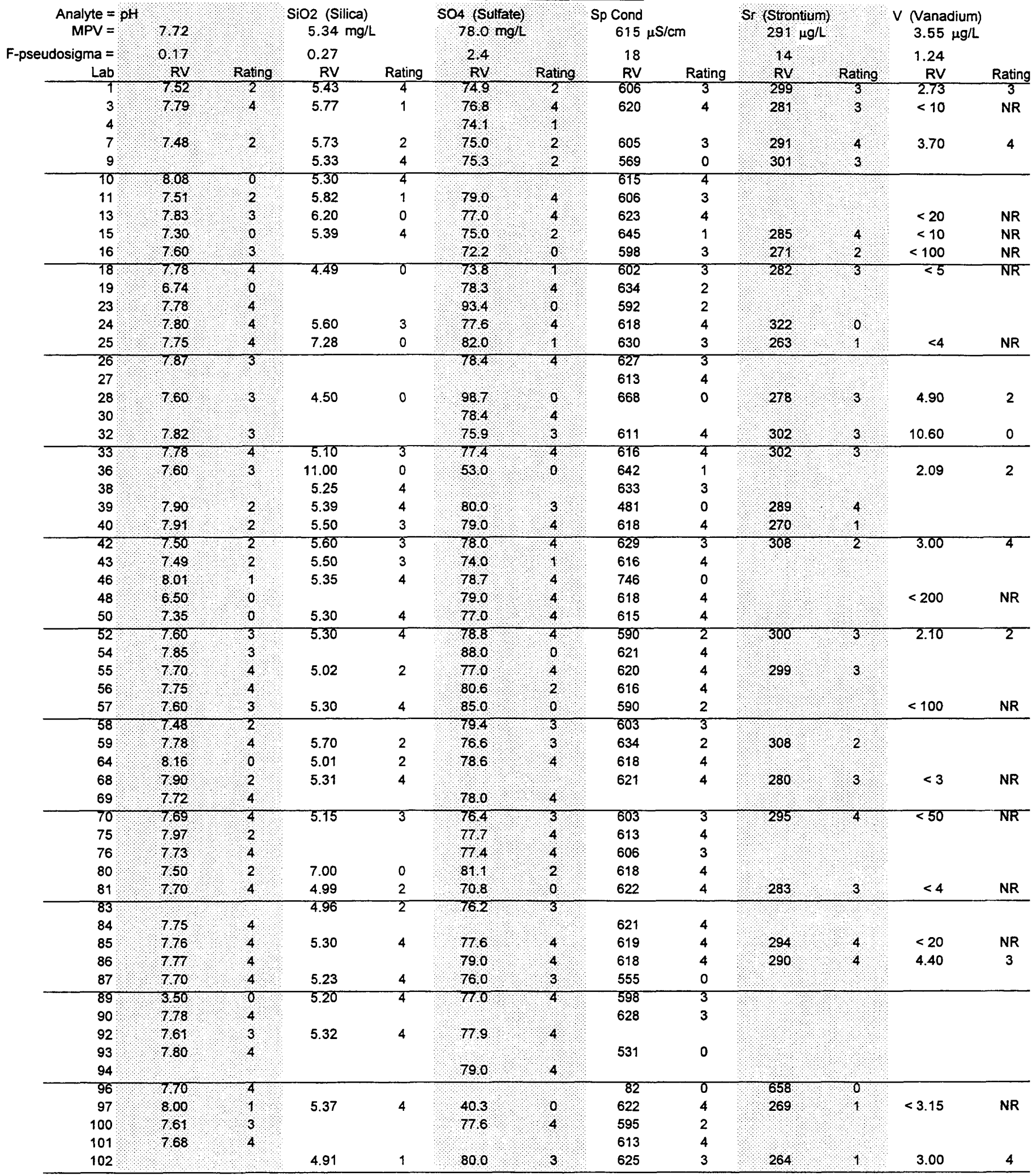


Table 6. -Laboratory performance ratings for standard reference water sample M-134 (major constituents) --Continued

\begin{tabular}{|c|c|c|c|c|c|c|c|c|c|c|c|c|}
\hline $\begin{aligned} \text { Analyte } & =\mathrm{pH} \\
\mathrm{MPV} & =\end{aligned}$ & 172 & & $\begin{array}{r}\mathrm{SiO} 2 \text { (Silica) } \\
5.34 \mathrm{mg} / \mathrm{L}\end{array}$ & & SO4 (Sulfate) & & $\begin{array}{r}\text { Sp Cond } \\
615\end{array}$ & & Sr (Stron & & $\begin{array}{c}V \text { (Vanadium) } \\
3.55 \mu \mathrm{g} / \mathrm{L}\end{array}$ & \\
\hline $\begin{array}{c}\text { F-pseudosigma }= \\
\text { Lab }\end{array}$ & 017 & Rating & $\begin{array}{r}0.27 \\
\text { RV } \\
\end{array}$ & Rating & $\begin{array}{l}2.4 \\
\mathrm{RV}\end{array}$ & Rating & $\begin{array}{r}18 \\
\text { RV } \\
\end{array}$ & Rating & $\begin{array}{r}14 \\
\text { RV }\end{array}$ & Rating & $\begin{array}{r}1.24 \\
\mathrm{RV} \\
\end{array}$ & Ratins \\
\hline & & & 4.70 & 0 & & & & & 285 & 4 & $<10$ & NR \\
\hline 105 & 17 & 4 & 5.63 & 2 & 803 & 3 & 618 & 4 & 316 & 1 & $<13$ & NF: \\
\hline 107 & 476 & 0 & 5.48 & 3 & & & 625 & 3 & & & & \\
\hline 109 & 678 & 0 & & & 757 & 3 & 596 & 2 & & & & \\
\hline 111 & 784 & 3 & 5.19 & 3 & 75.3 & 2 & 610 & 4 & & & & \\
\hline 114 & 760 & 3 & & & 82.0 & 1 & 583 & 1 & & & & \\
\hline 116 & & & 5.37 & 4 & 834 & 0 & & & 309 & 2 & & \\
\hline 118 & 750 & 2 & 5.40 & 4 & & & 612 & 4 & & & & \\
\hline 119 & 796 & 2 & 6.00 & 0 & 78.0 & 4 & 603 & 3 & & & & \\
\hline 121 & & & 5.30 & 4 & & & & & 300 & 3 & & \\
\hline 127 & 770 & 4 & 5.36 & 4 & 792 & 4 & 604 & 3 & 284 & 3 & $<4$ & NR \\
\hline 128 & 769 & 4 & 5.18 & 3 & 785 & 4 & 639 & 2 & & & $<5$ & NR \\
\hline 129 & 181 & 3 & & & 78.0 & 4 & 591 & 2 & & & & \\
\hline 132 & 773 & 4 & & & & & & & & & & \\
\hline 133 & & & & & & 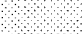 & & & & & & - \\
\hline 134 & 171 & 4 & 5.38 & 4 & 788 & 4 & 616 & 4 & 287 & 4 & 2.90 & 3 \\
\hline 136 & 784 & 3 & & & 791 & 4 & 621 & 4 & & & & \\
\hline 138 & 798 & 1 & 5.56 & 3 & 755 & 2 & & & 298 & 3 & 2.90 & 3 \\
\hline 140 & 752 & 2 & 5.54 & 3 & 815 & 2 & 626 & 3 & & & & \\
\hline 141 & 186 & 3 & 5.45 & 4 & 800 & 3 & 622 & 4 & & 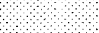 & $<10$ & NR \\
\hline 142 & 772 & 4 & 6.43 & 0 & 74.0 & 1 & 627 & 3 & 309 & 2 & 3.72 & 4 \\
\hline 143 & 782 & 3 & & & & & 607 & 4 & & & & \\
\hline 145 & 720 & 0 & 5.27 & 4 & 785 & 4 & 589 & 2 & 278 & 3 & 3.80 & 4 \\
\hline 146 & 736 & 0 & & & 123.0 & 0 & 636 & 2 & & & 2.60 & 3 \\
\hline 149 & 796 & 2 & 5.30 & 4 & 76.0 & 3 & & & & & & \\
\hline $151:$ & 767 & 4 & 5.30 & 4 & 79.0 & 4 & 615 & 4 & & & & \\
\hline 153 & 752 & 2 & & & 784 & 4 & 606 & 3 & & & & \\
\hline 154 & 819 & 0 & & & 74.8 & 2 & 606 & 3 & 284 & 3 & 11.80 & 0 \\
\hline 180 & 770 & 4 & & & 790 & 4 & 480 & 0 & & & $<4.1$ & NR \\
\hline 182 & 760 & 3 & 2.63 & 0 & 90.0 & 0 & 556 & 0 & 320 & 0 & 3.59 & 4 \\
\hline 183 & & & & & & & 555 & 0 & & & & \\
\hline 185 & & & & & & & & & & & & \\
\hline 190 & 791 & 2 & 5.56 & 3 & 792 & 4 & 623 & 4 & $<0,05$ & 0 & & \\
\hline 191 & & & & & 769 & 4 & & & 286 & 4 & & . \\
\hline 193 & & & & & 79.7 & 3 & 614 & 4 & & & & \\
\hline 194 & 755 & 3 & & & 76.9 & 4 & 570 & 0 & & & & \\
\hline 196 & 644 & 0 & & & 762 & 3 & 659 & 0 & & & & \\
\hline 197 & & & & & 79.9 & 3 & & & & & & \\
\hline 203 & 767 & 4 & 5.26 & 4 & 2410 & 0 & 610 & 4 & & & & \\
\hline 204 & $\begin{array}{r}729 \\
\end{array}$ & 0 & 5.96 & 0 & 812 & 2 & 58 & 0 & & & & \\
\hline 208 & & & & & 76.5 & 3 & & & & & & \\
\hline 210 & 811 & 0 & 5.89 & 0 & 780 & 4 & 590 & 2 & 291 & 4 & 3.50 & 4 \\
\hline 212 & 760 & 3 & 6.10 & 0 & 797 & 3 & 613 & 4 & 330 & 0 & 4.70 & 3 \\
\hline 213 & 778 & 4 & & & & & & & & & & \\
\hline 215 & 186 & 3 & 4.78 & 0 & 820 & 1 & 621 & 4 & & & & \\
\hline 219 & & & & & & & & & 340 & 0 & 3.90 & 4 \\
\hline 221 & 770 & 4 & & & & & & & & & & \\
\hline 224 & 770 & 4 & & & 783 & 4 & 590 & 2 & & & 1.80 & 2 \\
\hline 226 & & & 5.34 & 4 & 611 & 0 & & & & & & \\
\hline 230 & & & & & 76.2 & 3 & & & & & & \\
\hline 231 & & & 5.23 & 4 & 84.5 & 0 & & & & & & \\
\hline 234 & 789 & 2 & 5.30 & 4 & 804 & 3 & 620 & 4 & 294 & 4 & 2.02 & 2 \\
\hline 236 & 170 & 4 & 5.02 & 2 & 71 & 4 & 550 & 0 & 287 & 4 & 7.00 & 0 \\
\hline 237 & 820 & 0 & 55.00 & 0 & 770 & 4 & 639 & 2 & & & & \\
\hline 240 & 797 & 2 & & & 770 & 4 & 550 & 0 & & & & \\
\hline
\end{tabular}


Table 7. -Laboratory performance ratings for standard reference water sample N-45 (nutrients)

(MPV, most probable value; ug/L, micrograms per liter; mg/L, milligrams per liter; Lab, laboratory number; OLR, overall laboratory rating for all reported values; V/5, number of reported values of 5 possible values; RV, reported value; <, less than)

\begin{tabular}{|llll|}
\hline Rating & Absolute Z-value & Rating & Absolute Z-value \\
4 (Excellent) & $0.00-0.50$ & 1 (Questionable) & $1.51-2.00$ \\
3 (Good) & $0.51-1.00$ & $O$ (Poor) & greater than 2.00 \\
2 (Satisfactory) & $1.01-1.50$ & NR (Not Rated) & \\
\hline
\end{tabular}

\begin{tabular}{|c|c|c|c|c|c|c|c|c|c|c|c|c|}
\hline \multirow[b]{2}{*}{ Lab } & \multicolumn{4}{|c|}{ 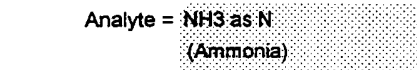 } & \multicolumn{2}{|c|}{$\begin{array}{l}\mathrm{NH} 3+\operatorname{Org} \mathrm{N} \text { as } \mathrm{N} \\
\text { (Ammonia+Organic } \mathrm{N} \text { ) } \\
0.300 \mathrm{mg} / \mathrm{L} \\
0.249\end{array}$} & \multicolumn{2}{|c|}{ 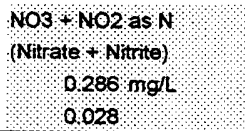 } & \multicolumn{2}{|c|}{$\begin{array}{l}\text { total P as P } \\
\text { (total Phosphorus) } \\
0.139 \mathrm{mg} / \mathrm{L} \\
0.012\end{array}$} & \multicolumn{2}{|c|}{ 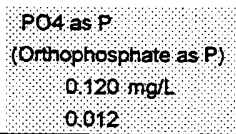 } \\
\hline & OLR & V/5 & सRV & Rating & RV & Rating & से RV & Rating & RV & Rating & Bद $\mathrm{RV}$ & Rating \\
\hline 1 & 2.8 & 4 & 0.054 & 4 & $<0.2$ & NR & 0214 & 0 & 0.130 & 3 & 0.123 & 4 \\
\hline 3 & 3.8 & 4 & 0.062 & 4 & $<1$ & NR & 0303 & 3 & 0.144 & 4 & 0,119 & 4 \\
\hline 7 & 4.0 & 2 & $<01$ & NR & & & $0: 280$ & 4 & 0.140 & 4 & & \\
\hline 9 & 3.0 & 5 & 0051 & 4 & 0.540 & 3 & 0307 & 3 & 0.161 & 1 & 0,17 & 4 \\
\hline 10 & 3.8 & 5 & 0054 & 4 & 0.340 & 4 & 0290 & 4 & 0.143 & 4 & 0126 & 3 \\
\hline 12 & 3.3 & 3 & & & & & 0290 & 4 & 0.130 & 3 & 0113 & 3 \\
\hline 13 & 1.3 & 4 & 0060 & 4 & & & 0,240 & 1 & 0.250 & 0 & 0060 & 0 \\
\hline 15 & 3.5 & 4 & 0052 & 4 & $<0.5$ & NR & 0312 & 3 & 0.139 & 4 & 0,126 & 3 \\
\hline 16 & 0.8 & 5 & 0174 & 0 & 0.406 & 4 & 0,401 & 0 & 0.097 & 0 & 0072 & 0 \\
\hline 18 & 3.2 & 5 & 0,054 & 4 & 0.224 & 4 & 0.028 & 0 & 0.137 & 4 & 0,125 & 4 \\
\hline 19 & 2.0 & 3 & & & & & 0300 & 4 & 0.120 & 1 & 0140 & 1 \\
\hline 21 & 4.0 & 5 & 0050 & 4 & 0.241 & 4 & 0280 & 4 & 0.142 & 4 & $0 / 119$ & 4 \\
\hline 22 & 4.0 & 13 & & & & & & & 0.144 & 4 & & \\
\hline 23 & 2.3 & 4 & 0.073 & 3 & & & 0281 & 4 & 0.112 & 0 & 0.137 & 2 \\
\hline 25 & 2.0 & 2 & $<0.05$ & NR & $<0.05$ & NR & 0280 & 4 & $<0.121$ & NR & 0.038 & 0 \\
\hline 26 & 2.3 & 3 & 0,083 & 2 & & & 0247 & 2 & & & 0131 & 3 \\
\hline 28 & 1.0 & 5 & 0,100 & 1 & 2.200 & 0 & 0,040 & 0 & 0.200 & 0 & 0120 & 4 \\
\hline 32 & 0.5 & 2 & 0096 & 1 & & & & & & & 0,095 & 0 \\
\hline 33 & 4.0 & 1 & $0: 060$ & 4 & & & & & & & & \\
\hline 36 & 2.3 & 4 & 0200 & 0 & $<0.5$ & NR & 0243 & 1 & 0.140 & 4 & 0120 & 4 \\
\hline 38 & 3.8 & 5 & 0,054 & 4 & 0.240 & 4 & 0,291 & 4 & 0.146 & 3 & 0116 & 4 \\
\hline 39 & 3.0 & 4 & 0.044 & 3 & & & 0288 & 4 & 0.145 & 3 & 0105 & 2 \\
\hline 42 & 2.0 & 3 & & & & & 0,250 & 2 & 0.153 & 2 & 0,135 & 2 \\
\hline 43 & 1.0 & 1 & & & & & 0340 & 1 & & & & \\
\hline 46 & 3.8 & 5 & 0053 & 4 & 0.260 & 4 & 0286 & 4 & 0.132 & 3 & 0,121 & 4 \\
\hline 48 & 1.2 & 5 & 0.050 & 4 & 6.400 & 0 & 0240 & 1 & 1.200 & 0 & 0136 & 1 \\
\hline 52 & 3.8 & 5 & 0,060 & 4 & 0.394 & 4 & 0286 & 4 & 0.149 & 3 & 0124 & 4 \\
\hline 53 & 2.7 & 3 & 0018 & 1 & & & 0,291 & 4 & & & 0,129 & 3 \\
\hline 55 & 3.2 & 5 & 0077 & 3 & 0.166 & 3 & 0297 & 4 & 0.147 & 3 & 0131 & 3 \\
\hline 56 & 2.3 & 4 & & & 0.150 & 3 & 02250 & 2 & 0.160 & 1 & 0130 & 3 \\
\hline 58 & 1.8 & 5 & 0,030 & 2 & 0.550 & 3 & 0660 & 0 & 0.260 & 0 & 0120 & 4 \\
\hline 59 & 2.4 & 5 & 0,050 & 4 & 0.300 & 4 & 0,230 & 1 & 0.200 & 0 & 0110 & 3 \\
\hline 60 & 2.0 & 3 & 0,093 & 1 & 0.570 & 2 & 0271 & 3 & & & & \\
\hline 64 & 4.0 & 4 & 0.060 & 4 & & & 0280 & 4 & 0.141 & 4 & 0125 & 4 \\
\hline 69 & 4.0 & 1 & & & & & 0.280 & 4 & & & & \\
\hline 70 & 3.3 & 4 & $<01$ & NR & 0.418 & 4 & 0247 & 2 & 0.134 & 4 & 0114 & 3 \\
\hline 75 & 3.5 & 4 & 0.055 & 4 & & & 0269 & 3 & 0.132 & 3 & 0,123 & 4 \\
\hline 76 & 3.0 & 2 & 0050 & 4 & & & 0244 & 2 & & & & \\
\hline 80 & 2.0 & 3 & 0250 & 0 & & & 0,260 & 3 & & & 0110 & 3 \\
\hline 83 & 2.7 & 3 & & . & & & 0290 & 4 & 0.100 & 0 & 0,124 & 4 \\
\hline 84 & 3.0 & 2 & 0049 & 3 & & & 0260 & 3 & & & & \\
\hline 85 & 3.6 & 5 & 0,054 & 4 & 0.320 & 4 & 0280 & 4 & 0.130 & 3 & 0114 & 3 \\
\hline 86 & 2.5 & 4 & 0081 & 3 & & & 0282 & 4 & 0.133 & 3 & 0157 & 0 \\
\hline 87 & 2.0 & 5 & 0,030 & 2 & 0.150 & 3 & 0.290 & 4 & 0.165 & 0 & 0,144 & 1 \\
\hline 88 & 1.0 & 3 & 0.048 & 3 & & & 0,496 & 0 & & & 0,194 & 0 \\
\hline 89 & 3.4 & 5 & 0070 & 4 & 0.587 & 2 & 0290 & 4 & 0.136 & 4 & 0126 & 3 \\
\hline 90 & 3.2 & 5 & 0,048 & 3 & 0.154 & 3 & 0,318 & 2 & 0.136 & 4 & 0124 & 4 \\
\hline 91 & 3.2 & 5 & 0,060 & 4 & 0.230 & 4 & 0280 & 4 & 0.160 & 1 & 0,110 & 3 \\
\hline 92 & 1.3 & 4 & 0.630 & 0 & & & 0284 & 4 & 0.194 & 0 & 0143 & 1 \\
\hline 93 & 4.0 & 1 & 0.050 & 4 & & & & & & & & \\
\hline 94 & 3.3 & 4 & 0070 & 4 & 0.110 & 3 & 0290 & 4 & 0.125 & 2 & & \\
\hline 96 & 3.6 & 5 & 0,045 & 3 & 0.165 & 3 & 0280 & 4 & 0.140 & 4 & 0,118 & 4 \\
\hline 97 & 3.0 & 5 & 0052 & 4 & 0.190 & 4 & 0,300 & 4 & 0.060 & 0 & 0110 & 3 \\
\hline 100 & 2.3 & 3 & 0040 & 3 & 1.310 & 0 & 0300 & 4 & & & & \\
\hline 102 & 1.8 & 5 & 0.080 & 3 & 0.080 & 3 & 0.230 & 1 & 0.120 & 1 & 0,100 & 1 \\
\hline 104 & 3.5 & 4 & & & 0.211 & 4 & 0291 & 4 & 0.139 & 4 & 0133 & 2 \\
\hline 105 & 2.4 & 5 & 0060 & 4 & 0.660 & 2 & 0.440 & 0 & 0.146 & 3 & 0127 & 3 \\
\hline 107 & 3.8 & 4 & 0.058 & 4 & & & 0,304 & 3 & 0.140 & 4 & 0121 & 4 \\
\hline 108 & 2.3 & 3 & & & & & 0270 & 3 & 0.600 & 0 & 0120 & 4 \\
\hline 111 & 3.3 & 3 & & & & & 0.266 & 3 & 0.141 & 4 & 0,130 & 3 \\
\hline
\end{tabular}


Table 7. -Laboratory performance ratings for standard reference water sample N-45 (nutrients) --Continued

\begin{tabular}{|c|c|c|c|c|c|c|c|c|c|c|c|c|}
\hline \multirow[b]{2}{*}{ Lab } & \multicolumn{4}{|c|}{ Analyte $=\begin{aligned} N H 3 \text { as } N \\
\\
(A m m o n a)\end{aligned}$} & \multicolumn{2}{|c|}{$\begin{array}{l}\mathrm{NH} 3+\operatorname{Org~N} \text { as } \mathrm{N} \\
\text { (Ammonia+Organic } \mathrm{N} \text { ) } \\
0.300 \mathrm{mg} / \mathrm{L} \\
0.249\end{array}$} & \multicolumn{2}{|c|}{ 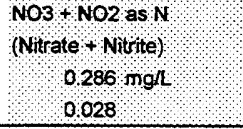 } & \multicolumn{2}{|c|}{$\begin{array}{l}\text { total P as P } \\
\text { (total Phosphorus) } \\
0.139 \mathrm{mg} / \mathrm{L} \\
0.012\end{array}$} & \multicolumn{2}{|c|}{ 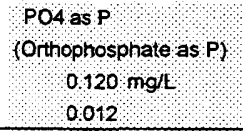 } \\
\hline & OLR & V/5 & $\mathrm{n} \mathrm{RV}$ & Rating & RV & Rating & म RV & Rating & RV & Rating & B RV & Rating \\
\hline 114 & 2.5 & 2 & $<01$ & $\mathrm{NR}$ & & & 02290 & 43 & 0.120 & 1 & & \\
\hline 118 & 2.6 & 5 & 0,100 & 1द & 0.320 & 4 & 0.240 & 1 & 0.130 & 3 & 0120 & 4 \\
\hline 119 & 3.4 & 5 & 0.090 & 2 & 0.230 & 4 & 0.300 & 4 & 0.140 & 4 & 0,110 & 3 \\
\hline 126 & 3.0 & 1 & & & & & 0.270 & 3 & & & & \\
\hline 127 & 3.4 & 5 & 0057 & 4 & 0.177 & 4 & 0266 & 3 & 0.133 & 3 & 0126 & 3 \\
\hline 128 & 3.0 & 5 & 0090 & 2 & 0.420 & 4 & 0,310 & 3 & 0.150 & 3 & 0130 & 3 \\
\hline 129 & 2.8 & 5 & 0147 & 0 & 0.162 & 3 & 0304 & 3 & 0.136 & 4 & 0,117 & 4 \\
\hline 132 & 3.3 & 4 & 0.050 & 4 & & & 0250 & 2 & 0.140 & 4 & 0.110 & 3 \\
\hline 133 & 3.2 & 5 & 0076 & 3 & 0.220 & 4 & 0230 & 1 & 0.140 & 4 & 0120 & 4 \\
\hline 134 & 3.4 & 5 & 0070 & 4 & 0.200 & 4 & 0.300 & 4 & 0.120 & 1 & 0.120 & 4 \\
\hline 136 & 4.0 & 1 & 0,069 & 4 & & & & & & & & \\
\hline 138 & 3.6 & 5 & 0.052 & 4 & 0.284 & 4 & 02294 & 4 & 0.133 & 3 & 0113 & 3 \\
\hline 140 & 2.4 & 5 & 0080 & 3 & 0.350 & 4 & 0.290 & 4 & 0.100 & 0 & 0.100 & 1 \\
\hline 141 & 2.5 & 4 & 0101 & 1 & $<1$ & NR & 0284 & 4 & 0.140 & 4 & 0.140 & 1 \\
\hline 142 & 1.4 & 5 & 0.018 & 1 & 0.536 & 3 & 0,306 & 3 & 0.173 & 0 & 0151 & 0 \\
\hline 143 & 3.8 & 5 & $0: 040$ & 3 & 0.300 & 4 & 0.281 & 4 & 0.137 & 4 & 0118 & 4 \\
\hline 145 & 3.2 & 5 & 0,050 & 4 & 0.350 & 4 & 0240 & 1 & 0.150 & 3 & 0120 & 4 \\
\hline 146 & 4.0 & 3 & 0.055 & 4 & & & 0.279 & 4 & & & 0,121 & 4 \\
\hline 149 & 4.0 & 2 & 0.050 & 4 & & & & & 0.136 & 4 & & \\
\hline 151 & 2.8 & 4 & 0040 & 3 & & & 02290 & 4 & 0.160 & 1 & 0130 & 3 \\
\hline 154 & 3.3 & 4 & 0056 & 4 & & & 0270 & 3 & 0.127 & 2 & 0119 & 4 \\
\hline 180 & 3.6 & 5 & 0051 & 4 & 0.194 & 4 & 0298 & 4 & 0.153 & 2 & 0124 & 4 \\
\hline 182 & 0.0 & 3 & 0.010 & 0 & & & & & 0.180 & 0 & 0480 & 0 \\
\hline 183 & 1.0 & 3 & 0080 & 3 & & & 1000 & 0 & & & 0270 & 0 \\
\hline 185 & 2.8 & 5 & 0053 & 4 & 0.611 & 2 & 0,300 & 3 & 0.127 & 2 & 0114 & 3 \\
\hline 190 & 3.4 & 5 & 0.080 & 3 & 0.150 & 3 & 0282 & 4 & 0.139 & 4 & 0131 & 3 \\
\hline 191 & 2.0 & 3 & & & & & 0250 & 2 & 0.134 & 4 & 0075 & 0 \\
\hline 193 & 1.5 & 2 & & & & & 0320 & 2 & & & 0142 & 1 \\
\hline 194 & 2.7 & 3 & 0160 & 0 & 0.210 & 4 & 02290 & 4 & & & & \\
\hline 196 & 3.0 & 2 & & & & & 02251 & 2 & & & 0,115 & 4 \\
\hline 197 & 4.0 & 2 & 0052 & 4 & & & 0.296 & 4 & & & & \\
\hline 198 & 3.2 & 5 & 0055 & 4 & 0.324 & 4 & 0,306 & 3 & 0.156 & 2 & 0128 & 3 \\
\hline 203 & 3.0 & 5 & 0066 & 4 & 0.316 & 4 & 0.215 & 0 & 0.133 & 3 & 0.125 & 4 \\
\hline 208 & 4.0 & 2 & & & & & 0.289 & 4 & & & 0120 & 4 \\
\hline 209 & 2.7 & 3 & 0.060 & 4 & 0.160 & 3 & 0.330 & 1 & & & & \\
\hline 210 & 0.0 & 3 & 0300 & 0 & 1.300 & 0 & $<05$ & NR & $<0.25$ & NR & 0146 & 0 \\
\hline 212 & 3.0 & 4 & 0110 & 0 & $<0.5$ & NR & 02290 & 4 & 0.140 & 4 & 0120 & 4 \\
\hline 215 & 2.8 & 4 & 0,080 & 3 & & & 0.290 & 4 & 0.130 & 3 & 0100 & 1 \\
\hline 221 & 2.6 & 5 & 0060 & 4 & 0.560 & 2 & 0,350 & 0 & 0.139 & 4 & 0110 & 3 \\
\hline 224 & 1.8 & 5 & 0.560 & 0 & 0.580 & 2 & 0319 & 2 & 0.126 & 2 & 0109 & 3 \\
\hline 226 & 3.3 & 4 & 0072 & 3 & 0.428 & 3 & 0304 & 3 & 0.139 & 4 & & \\
\hline 227 & 4.0 & 2 & & & & & & & 0.139 & 4 & 0120 & 4 \\
\hline 231 & 3.0 & 5 & 0050 & 4 & 0.230 & 4 & 0260 & 3 & 0.110 & 0 & 0120 & 4 \\
\hline 234 & 2.3 & 4 & 0076 & 3 & & & 0262 & 3 & 0.148 & 3 & 0.084 & 0 \\
\hline 240 & 0.0 & 5 & 1960 & 0 & 3.040 & 0 & 0.530 & 0 & 0.016 & 0 & $<001$ & 0 \\
\hline 241 & 3.4 & 5 & 0.053 & 4 & 0.271 & 4 & 0256 & 2 & 0.136 & 4 & 0127 & 3 \\
\hline
\end{tabular}


Table 8. -Laboratory performance ratings for standard reference water sample $\mathrm{N}-46$ (nutrients)

(MPV,most probable value; ug/L, micrograms per liter, mg/L, milligrams per liter, Lab, laboratory number, OLR, overall laboratory rating for all reported values; V/5, number of reported values of 5 possible values; RV, reported value; <, less than)

\begin{tabular}{|llll|}
\hline Rating & Absolute Z-value & Rating & Absolute Z-value \\
4 (Excellent) & $0.00-0.50$ & 1 (Questionable) & $1.51-2.00$ \\
3 (Good) & $0.51-1.00$ & $O$ (Poor) & greater than 2.00 \\
2 (Satisfactory) & $1.01-1.50$ & NR (Not Rated) & \\
\hline
\end{tabular}

\begin{tabular}{|c|c|c|c|c|c|c|c|c|c|c|c|c|}
\hline \multirow[b]{2}{*}{ Lab } & \multirow{2}{*}{\multicolumn{2}{|c|}{$\begin{array}{r}\text { Analyte }= \\
\text { MPV }= \\
\text { F-pseudosigma }= \\
\text { OLR V/5 }\end{array}$}} & \multirow{2}{*}{$\begin{array}{l}\text { NH } 3 \text { as N } \\
\text { Ammona) } \\
\text { to } 04 \\
0,09\end{array}$} & \multirow{2}{*}{ क } & \multicolumn{2}{|c|}{$\begin{array}{l}\mathrm{NH} 3+\operatorname{Org} \mathrm{N} \text { as } \mathrm{N} \\
\text { (Ammonia+Organic } \mathrm{N}) \\
1.81 \mathrm{mg} / \mathrm{L} \\
0.30 \\
\end{array}$} & \multicolumn{2}{|c|}{ 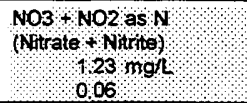 } & \multicolumn{2}{|c|}{$\begin{array}{l}\text { total } P \text { as } P \\
\text { (total Phosphorus) } \\
1.23 \mathrm{mg} / \mathrm{L} \\
0.06\end{array}$} & \multicolumn{2}{|c|}{ 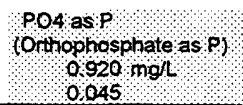 } \\
\hline & & & & & RV & Rating & सRV & Ráting & RV & Rating & R RY & Rating \\
\hline 1 & 3.6 & 5 & 1082 & 4 & 1.68 & 4 & 18 & 3 & 1.20 & 3 & 0920 & 4 \\
\hline 3 & 3.8 & 5 & 1,100 & 3 & 1.80 & 4 & 125 & 4 & 1.25 & 4 & 0,909 & 4 \\
\hline 7 & 1.8 & 4 & 0.980 & 3 & & & 126 & 3 & 1.43 & 0 & 0.850 & 1 \\
\hline 9 & 2.7 & 3 & 1050 & 4 & 1.92 & 4 & & & 1.37 & 0 & & \\
\hline 10 & 3.0 & 5 & 1040 & 4 & 1.86 & 4 & 124 & 4 & 1.27 & 3 & 1020 & 0 \\
\hline 12 & 2.3 & 4 & 1000 & 4 & 2.40 & 1 & 128 & 3 & & & 0840 & 1 \\
\hline 13 & 2.5 & 4 & 1060 & 4 & & & 119 & 3 & 1.27 & 3 & 0750 & 0 \\
\hline 15 & 3.4 & 5 & 1100 & 3 & 1.67 & 4 & 130 & 2 & 1.24 & 4 & 0,910 & 4 \\
\hline 16 & 1.6 & 5 & 0,928 & 2 & 2.67 & 0 & 128 & 3 & 1.08 & 0 & 0,896 & 3 \\
\hline 18 & 4.0 & 5 & 1,060 & 4 & 1.70 & 4 & 124 & 4 & 1.25 & 4 & 0,941 & 4 \\
\hline 19 & 3.5 & 2 & 0.970 & 3 & & & 121 & 4 & & & & \\
\hline 22 & 3.0 & 1 & & & & & & & 1.30 & 3 & & \\
\hline 23 & 3.8 & 5 & 1040 & 4 & 2.08 & 3 & 123 & 4 & 1.26 & 4 & 0.930 & 4 \\
\hline 25 & 1.3 & 3 & $<0.05$ & 0 & $<0.05$ & 0 & 115 & 2 & 1.15 & 2 & 0286 & 0 \\
\hline 26 & 2.3 & 3 & 1,020 & 4 & & & 117 & 2 & & & 0,990 & 1 \\
\hline 28 & 2.8 & 5 & 1.040 & 4 & 5.50 & 0 & 1.24 & 4 & 1.23 & 4 & 0970 & 2 \\
\hline 30 & 1.5 & 2 & & & & & 126 & 3 & & & 2,730 & 0 \\
\hline 33 & 1.0 & 1 & 1190 & 1 & & & & & & & & \\
\hline 36 & 0.0 & 5 & 0,520 & 0 & 1.10 & 0 & 097 & 0 & 1.38 & 0 & 1020 & 0 \\
\hline 38 & 2.8 & 5 & 1129 & 3 & 4.03 & 0 & 124 & 4 & 1.27 & 3 & 0,908 & 4 \\
\hline 42 & 3.3 & 3 & & & & & 125 & 4 & 1.27 & 3 & 0,954 & 3 \\
\hline 43 & 2.0 & 1 & & & & & 130 & 2 & & & & \\
\hline 46 & 3.6 & 5 & 1013 & 4 & 1.93 & 4 & 1,31 & 2 & 1.21 & 4 & 0,930 & 4 \\
\hline 48 & 1.4 & 5 & 1060 & 4 & 9.40 & 0 & 110 & 0 & 2.60 & 0 & 0044 & 3 \\
\hline 52 & 2.2 & 5 & $0: 595$ & 0 & 1.96 & 3 & 119 & 3 & 1.35 & 1 & 0,916 & 4 \\
\hline 53 & 2.7 & 3 & 1019 & 4 & & & 128 & 3 & & & 1003 & 1 \\
\hline 55 & 3.5 & 2 & 1090 & 3 & & & 124 & 4 & & & & \\
\hline 56 & 2.0 & 4 & & & 1.67 & 4 & 108 & 0 & 1.04 & 0 & 0,920 & 4 \\
\hline 57 & 1.4 & 5 & 1000 & 4 & 9.10 & 0 & 090 & 0 & 1.30 & 2 & 1000 & 1 \\
\hline 58 & 0.0 & 5 & 0.550 & 0 & 2.59 & 0 & 067 & 0 & 0.66 & 0 & 0,470 & 0 \\
\hline 59 & 3.2 & 5 & 1020 & 4 & 1.60 & 3 & 123 & 4 & 1.20 & 3 & 0870 & 2 \\
\hline 60 & 3.6 & 5 & 1115 & 3 & 1.82 & 4 & 129 & 3 & 1.24 & 4 & 0905 & 4 \\
\hline 64 & 3.5 & 4 & 1060 & 4 & & & 119 & 3 & 1.27 & 3 & 0,940 & 4 \\
\hline 68 & 2.8 & 4 & 1040 & 4 & 1.62 & 3 & 127 & 3 & 1.14 & 1 & & \\
\hline 69 & 2.0 & 1 & & & & & 130 & 2 & & & & \\
\hline 70 & 3.0 & 5 & 1010 & 4 & 1.84 & 4 & 117 & 2 & 1.33 & 1 & 0908 & 4 \\
\hline 75 & 3.5 & 4 & 1080 & 4 & & & 120 & 3 & 1.18 & 3 & 0.916 & 4 \\
\hline 76 & 3.5 & 2 & 0.997 & 4 & & & 119 & 3 & & & & \\
\hline 80 & 1.0 & 3 & 0.400 & 0 & & & 1,05 & 0 & & & 0,960 & 3 \\
\hline 83 & 3.0 & 3 & & & & & 134 & 1 & 1.21 & 4 & 0930 & 4 \\
\hline 84 & 1.0 & 3 & 0830 & 0 & & & 117 & 2 & & & 0840 & 1 \\
\hline 85 & 3.2 & 5 & 1070 & 4 & 2.00 & 3 & 1,22 & 4 & 1.17 & 2 & 0.876 & 3 \\
\hline 86 & 2.3 & 4 & 1130 & 3 & & & 127 & 3 & 1.27 & 3 & 1050 & 0 \\
\hline 87 & 2.8 & 5 & 0,990 & 3 & 1.73 & 4 & 1,22 & 4 & 1.51 & 0 & 0,944 & 3 \\
\hline 89 & 3.2 & 5 & 1100 & 3 & 1.91 & 4 & 127 & 3 & 1.20 & 3 & 0,890 & 3 \\
\hline 90 & 2.6 & 5 & 1070 & 4 & 1.67 & 4 & 130 & 2 & 1.30 & 2 & 0.990 & 1 \\
\hline 91 & 2.0 & 5 & 0940 & 2 & 1.85 & 4 & 16 & 2 & 1.30 & 2 & 1020 & 0 \\
\hline 92 & 2.0 & 4 & 1320 & 0 & & & 130 & 2 & 1.31 & 2 & 0019 & 4 \\
\hline 93 & 4.0 & 1 & 1040 & 4 & & & & & & & & \\
\hline 94 & 4.0 & 4 & 1050 & 4 & 1.73 & 4 & 122 & 4 & 1.24 & 4 & & \\
\hline 96 & 3.2 & 5 & 0972 & 3 & 1.77 & 4 & 129 & 2 & 1.26 & 4 & 0892 & 3 \\
\hline 97 & 4.0 & 5 & 1020 & 4 & 1.73 & 4 & 125 & 4 & 1.21 & 4 & 0020 & 4 \\
\hline 100 & 2.7 & 3 & 1000 & 4 & 2.73 & 0 & 124 & 4 & & & & \\
\hline 102 & 1.4 & 5 & 1580 & 0 & 1.78 & 4 & 108 & 0 & 1.18 & 3 & 0,720 & 0 \\
\hline 104 & , 4.0 & 4 & & & 1.78 & 4 & 122 & 4 & 1.25 & 4 & 0,919 & 4 \\
\hline 105 & 3.6 & 5 & 1100 & 3 & 1.87 & 4 & 128 & 3 & 1.24 & 4 & 0930 & 4 \\
\hline 107 & 1.8 & 4 & 1150 & 2 & & & 134 & 1 & 1.35 & 1 & 0.945 & 3 \\
\hline 108 & 0.4 & 5 & 8000 & 0 & 4.41 & 0 & 1,37 & 0 & 1.10 & 0 & 0,980 & 2 \\
\hline 111 & 3.8 & 4 & 1030 & 4 & & & 123 & 4 & 1.27 & 3 & 0926 & 4 \\
\hline 114 & 2.7 & 3 & 0.970 & 3 & & & 121 & 4 & 1.12 & 1 & & \\
\hline
\end{tabular}


Table 8. -Laboratory performance ratings for standard reference water sample N-46 (nutrients) --Continued

\begin{tabular}{|c|c|c|c|c|c|c|c|c|c|c|c|c|}
\hline \multirow[b]{2}{*}{ Lab } & F-pse & $\begin{array}{l}\text { latyte = } \\
\text { MPV = } \\
\text { igma = }\end{array}$ & 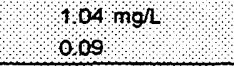 & & \multicolumn{2}{|c|}{$\begin{array}{c}\mathrm{NH} 3+\operatorname{Org~N} \text { as } \mathrm{N} \\
\text { (Ammonia+Organic N) } \\
1.81 \mathrm{mg} / \mathrm{L} \\
0.30\end{array}$} & \multicolumn{2}{|c|}{$\begin{array}{l}\mathrm{NO} 3 \text { + NO2 as N } \\
\text { (Nitate } \mathrm{N} \text { trite) } \\
123 \mathrm{mg}, \mathrm{l}\end{array}$} & \multicolumn{2}{|c|}{$\begin{array}{l}\text { total } P \text { as } P \\
\text { (total Phosphorus) } \\
1.23 \mathrm{mg} / \mathrm{L} \\
0.06\end{array}$} & \multicolumn{2}{|c|}{$\begin{array}{l}\text { PO4 as P } \\
\text { (Oithophosphate as P) } \\
\quad 0920 \mathrm{mg} / \mathrm{L}\end{array}$} \\
\hline & OLR & V/5 & R RV & Rating & RV & Rating & B RV & Rating & RV & Rating & B RV & Rating \\
\hline 118 & 2.6 & 5 & 170 & 2 & 2.10 & 3 & 117 & 2 & 1.30 & 2 & 0,920 & 4 \\
\hline 119 & 3.4 & 5 & 0970 & 3 & 1.70 & 4 & 116 & 2 & 1.22 & 4 & 0.910 & 4 \\
\hline 127 & 3.2 & 5 & 1540 & 0 & 1.80 & 4 & 124 & 4 & 1.25 & 4 & 0.916 & 4 \\
\hline 128 & 3.0 & 5 & 1010 & 4 & 1.77 & 4 & 107 & 0 & 1.20 & 3 & 0,920 & 4 \\
\hline 129 & 2.8 & 5 & 1109 & 3 & 2.00 & 3 & 124 & 4 & 1.20 & 3 & 0,850 & 1 \\
\hline 132 & 1.3 & 4 & 0770 & 0 & & & 109 & 0 & 1.29 & 3 & 0,980 & 2 \\
\hline 133 & 2.2 & 5 & 0,565 & 0 & 0.93 & 0 & 124 & 4 & 1.20 & 3 & 0.910 & 4 \\
\hline 134 & 3.2 & 5 & 1100 & 3 & 1.80 & 4 & 130 & 2 & 1.20 & 3 & 0,920 & 4 \\
\hline 138 & 3.8 & 5 & 1060 & 4 & 1.79 & 4 & 122 & 4 & 1.19 & 3 & 0019 & 4 \\
\hline 140 & 3.0 & 5 & 1050 & 4 & 1.81 & 4 & 124 & 4 & 1.43 & 0 & 0.960 & 3 \\
\hline 141 & 3.0 & 5 & 1060 & 4 & 0.94 & 0 & 121 & 4 & 1.22 & 4 & 0,890 & 3 \\
\hline 142 & 1.8 & 5 & 0.978 & 3 & 1.89 & 4 & 131 & 2 & 1.54 & 0 & 1060 & 0 \\
\hline 143 & 3.2 & 5 & 1030 & 4 & 1.50 & 2 & 115 & 2 & 1.23 & 4 & 0.926 & 4 \\
\hline 145 & 2.6 & 5 & 1060 & 4 & 1.72 & 4 & 108 & 0 & 1.16 & 2 & 0,960 & 3 \\
\hline 146 & 1.7 & 3 & 0,114 & 0 & & & 112 & 1 & & & 0936 & 4 \\
\hline 149 & 2.0 & 3 & 0940 & 2 & & & 125 & 4 & 1.08 & 0 & & \\
\hline 151 & 2.5 & 4 & 1,040 & 4 & & & 127 & 3 & 1.32 & 2 & 1000 & 1 \\
\hline 154 & 3.0 & 5 & 1070 & 4 & 1.68 & 4 & 124 & 4 & 1.14 & 1 & 0863 & 2 \\
\hline 180 & 3.2 & 5 & 1070 & 4 & 1.58 & 3 & 126 & 3 & 1.32 & 2 & 0,899 & 4 \\
\hline 182 & 1.7 & 3 & 0880 & 1 & & & & & 1.26 & 4 & 3350 & 0 \\
\hline 185 & 1.8 & 4 & & & 3.23 & 0 & 124 & 4 & 1.09 & 0 & 0.957 & 3 \\
\hline 190 & 3.2 & 5 & 1000 & 4 & 2.27 & 1 & 122 & 4 & 1.27 & 3 & 0,932 & 4 \\
\hline 191 & 3.0 & 3 & & & & & 121 & 4 & 1.17 & 2 & 0,879 & 3 \\
\hline 193 & 1.0 & 2 & & & & & 131 & 2 & & & 1300 & 0 \\
\hline 194 & 2.5 & 4 & 1,260 & 0 & 1.65 & 3 & 119 & 3 & 1.21 & 4 & & \\
\hline 197 & 4.0 & 2 & 1020 & 4 & & & 1.22 & 4 & & & & \\
\hline 198 & 2.8 & 5 & 0.900 & 1 & 1.75 & 4 & 120 & 3 & 1.16 & 2 & 0,930 & 4 \\
\hline 203 & 1.4 & 5 & 0506 & 0 & 1.91 & 4 & 1,17 & 3 & 1.07 & 0 & 0.578 & 0 \\
\hline 204 & 1.0 & 1 & 1182 & 1 & & & & & & & & \\
\hline 209 & 2.3 & 3 & 1050 & 4 & 1.58 & 3 & 1.42 & 0 & & & & \\
\hline 210 & 2.8 & 5 & 1100 & 3 & 2.10 & 3 & 1.20 & 3 & 1.20 & 3 & 0968 & 2 \\
\hline 212 & 2.8 & 5 & 1100 & 3 & 1.50 & 2 & 1,30 & 2 & 1.20 & 3 & 0,930 & 4 \\
\hline 215 & 3.0 & 4 & 1120 & 3 & & & 122 & 4 & 1.24 & 4 & $\$ 010$ & 1 \\
\hline 221 & 3.0 & 5 & 1040 & 4 & 1.79 & 4 & 146 & 0 & 1.20 & 3 & 0900 & 4 \\
\hline 224 & 2.0 & 5 & 0,950 & 3 & 2.85 & 0 & 217 & 0 & 1.23 & 4 & 0,960 & 3 \\
\hline 226 & 1.8 & 4 & 1034 & 4 & 2.56 & 0 & 126 & 3 & 1.72 & 0 & & \\
\hline 227 & 2.5 & 2 & & & & & & & 1.20 & 3 & 0,972 & 2 \\
\hline 231 & 3.4 & 5 & 0960 & 3 & 1.75 & 4 & 121 & 4 & 1.17 & 2 & 0,900 & 4 \\
\hline 234 & 2.0 & 4 & 1100 & 3 & & & 1.20 & 3 & 0.98 & 0 & 0.867 & 2 \\
\hline 237 & 1.5 & 2 & 0.590 & 0 & & & 120 & 3 & & & & \\
\hline 240 & 0.2 & 5 & 2700 & 0 & 5.10 & 0 & 107 & 0 & 0.12 & 0 & 0840 & 1 \\
\hline 241 & 2.4 & 5 & 0,970 & 3 & 3.80 & 0 & 119 & 3 & 1.26 & 4 & 0,864 & 2 \\
\hline
\end{tabular}


Table 9. -Laboratory performance ratings for standard reference water sample P-24 (low ionic strength)

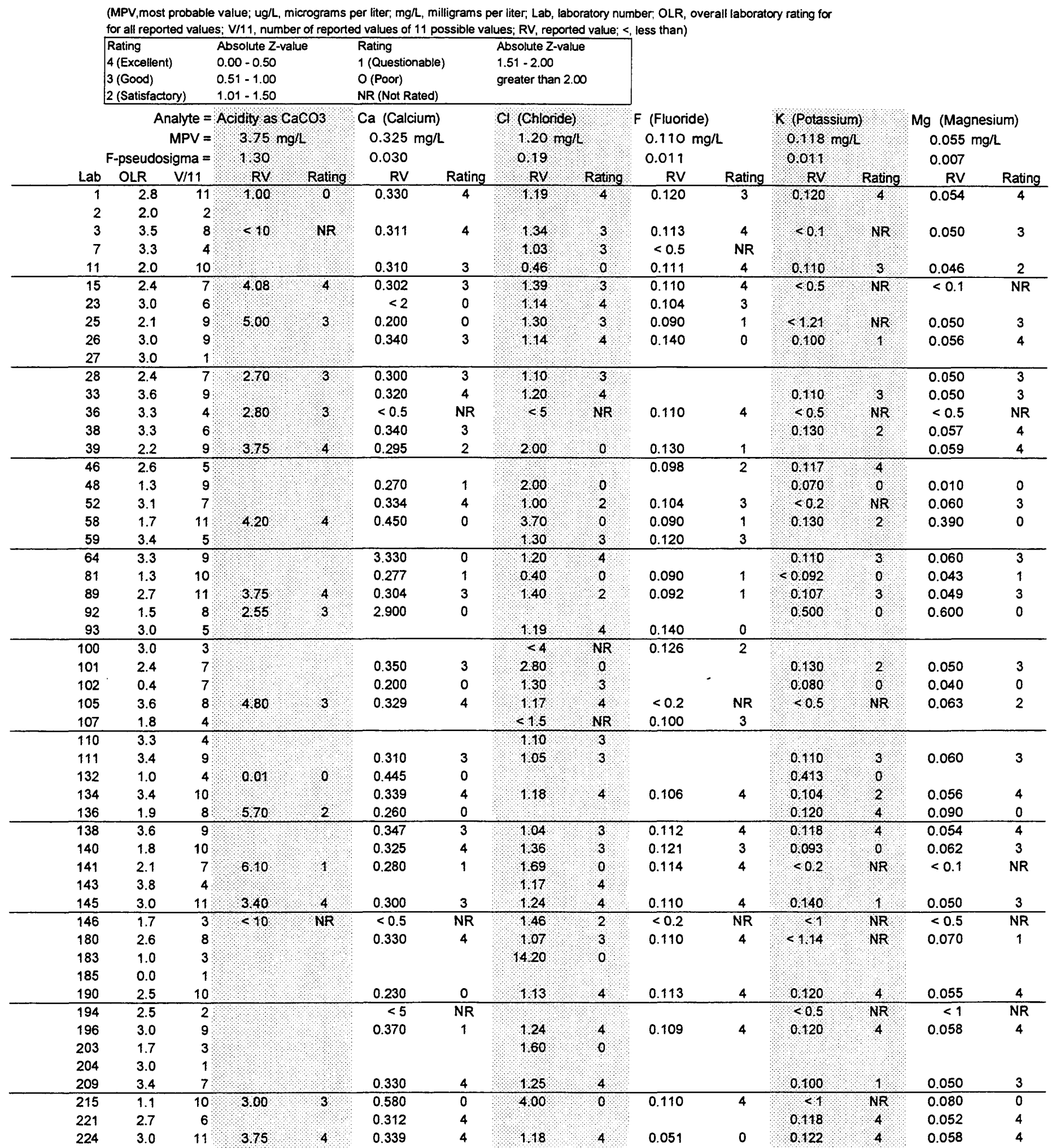


Table 9. -Laboratory performance ratings for standard reference water sample P-24 (low ionic strength) --Continued

(MPV,most probable value; uglL, micrograms per liter; $\mathrm{mg} / \mathrm{L}$, milligrams per liter, Lab, laboratory number, OLR, overall laboratory rating for all reported values; V/11, number of reported values of 11 possible values; RV, reported value; <, less than)

\begin{tabular}{llll}
\hline Rating & Absolute Z-value & Rating & Absolute Z-value \\
4 (Excellent) & $0.00-0.50$ & 1 (Questionable) & $1.51-2.00$ \\
3 (Good) & $0.51-1.00$ & $O$ (Poor) & greater than 2.00 \\
2 (Satisfactory) & $1.01-1.50$ & NR (Not Rated) &
\end{tabular}

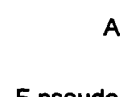

Analyte $=\mathrm{Na}$ (Sodium)

MPV =

0246 mgl

4.73

-pseudosigma

0.025

0.13

NR (Not Rated)

\begin{tabular}{|c|c|c|c|c|c|c|c|c|c|c|}
\hline $\mathrm{Lab}$ & RV & Rating & RV & Rating & RV & Rating & RV & Rating & RV & Rating \\
\hline 1 & 0267 & 3 & 4.92 & 2 & 0190 & 0 & 0.320 & 4 & 12.1 & 3 \\
\hline 2 & & & 4.52 & 1 & & & & & 12.4 & 3 \\
\hline 3 & 0250 & 4 & 4.72 & 4 & 0030 & 3 & $<1$ & NR & 14.6 & 3 \\
\hline 7 & & & 4.83 & 3 & < 016 & NR & 0.650 & 3 & 13.8 & 4 \\
\hline 11 & 0260 & 3 & 4.46 & 1 & 0,040 & 0 & 1.650 & 0 & 12.8 & 4 \\
\hline 15 & $<05$ & NR & 5.90 & 0 & 0.038 & 0 & $<0.5$ & NR & 14.8 & 3 \\
\hline 23 & $<0,5$ & NR & 4.78 & 4 & 0,030 & 3 & $<2.5$ & NR & 12.6 & 4 \\
\hline 25 & 0,245 & 4 & 4.78 & 4 & 0009 & o & $<5$ & NR & 16.0 & 1 \\
\hline 26 & 0,230 & 3 & 4.77 & 4 & $<0,1$ & NR & 0.330 & 4 & 13.8 & 4 \\
\hline 27 & & & & & & & & & 14.3 & 3 \\
\hline 28 & & & 4.90 & 2 & 0.030 & 3 & & & 660 & 0 \\
\hline 33 & 0240 & 4 & 4.72 & 4 & 0,030 & 3 & 0.300 & 4 & 14.4 & 3 \\
\hline 36 & $<0,5$ & $N R$ & 4.60 & 3 & $<0,025$ & $N R$ & $<5$ & NR & 11.8 & 3 \\
\hline 38 & 0.230 & 3 & & & 0.028 & 4 & & & 13.3 & 4 \\
\hline 39 & 0.244 & 4 & 4.80 & 3 & 0,022 & 1 & & & 10.4 & 1 \\
\hline 46 & & & 5.00 & 1 & 0.026 & 3 & & & 14.3 & 3 \\
\hline 48 & 0250 & 4 & 5.20 & 0 & 0031 & 3 & 2.000 & 0 & 12.5 & 4 \\
\hline 52 & $<0,3$ & $\mathrm{NR}$ & 4.70 & 4 & 0026 & 3 & $<10$ & NR & 12.0 & 3 \\
\hline 58 & 0,200 & 1 & 4.69 & 4 & 0.030 & 3 & 5.350 & 0 & 13.7 & 4 \\
\hline 59 & & & 4.65 & 3 & $<005$ & $\mathrm{NR}$ & 0.310 & 4 & 12.6 & 4 \\
\hline 64 & 0240 & 4 & 4.73 & 4 & 0,028 & 4 & 0.320 & 4 & 13.1 & 4 \\
\hline 81 & 0151 & 0 & 4.70 & 4 & 0.030 & 3 & 5.100 & 0 & 12.0 & 3 \\
\hline 89 & 02243 & 4 & 4.50 & 1 & 0030 & 3 & 0.320 & 4 & 11.2 & 2 \\
\hline 92 & 1000 & 0 & 4.88 & 2 & 0.026 & 3 & 0.160 & 4 & & \\
\hline 93 & & & 4.70 & 4 & & & 0.780 & 3 & 13.0 & 4 \\
\hline 100 & & & 4.79 & 4 & & & $<7$ & NR & 12.0 & 3 \\
\hline 101 & 0270 & 3 & 4.54 & 2 & & & & & 13.0 & 4 \\
\hline 102 & 0100 & 0 & & & 0,015 & 0 & 2.000 & 0 & & \\
\hline 105 & 0246 & 4 & 4.73 & 4 & 0,027 & 4 & $<1$ & NR & 12.8 & 4 \\
\hline 107 & & & 7.81 & 0 & 0.033 & 2 & & & 14.9 & 2 \\
\hline 110 & & & 4.72 & 4 & & & 0.335 & 4 & 15.0 & 2 \\
\hline 111 & 0250 & 4 & 4.85 & 3 & 0027 & 4 & 0.300 & 4 & 13.6 & 4 \\
\hline 132 & & & 4.74 & 4 & & & & & & \\
\hline 134 & $0 / 274$ & 2 & 4.75 & 4 & 0,030 & 3 & 0.348 & 4 & 14.2 & 3 \\
\hline 136 & 0.230 & 3 & 4.40 & 0 & 0030 & 3 & & & 14.4 & 3 \\
\hline 138 & 0252 & 4 & 4.61 & 3 & 0.026 & 3 & 0.280 & 4 & & \\
\hline 140 & 0340 & 0 & 4.38 & 0 & 0.015 & 0 & 1.000 & 2 & 14.6 & 3 \\
\hline 141 & 0260 & 3 & 4.90 & 2 & $<005$ & $\mathrm{NR}$ & $<10$ & NR & 13.2 & 4 \\
\hline 143 & & & 4.72 & 4 & 0.028 & 4 & & & 12.0 & 3 \\
\hline 145 & 0,220 & 2 & 4.70 & 4 & 0.040 & 0 & 0.240 & 4 & 14.0 & 4 \\
\hline 146 & $<05$ & $\mathrm{NR}$ & 3.54 & 0 & $<005$ & NR & $<5$ & $\overline{N R}$ & 14.3 & 3 \\
\hline 180 & 0,271 & 2 & 4.70 & 4 & 0.025 & 3 & $<2.5$ & NR & 10.0 & 0 \\
\hline 183 & & & & & & & 1.600 & 0 & 12.0 & 3 \\
\hline 185 & & & & & 0,019 & 0 & & & & \\
\hline 190 & 0.290 & 1 & 4.48 & 1 & 0,002 & 0 & 0.315 & 4 & 14.2 & 3 \\
\hline 194 & $\begin{array}{l}<5 \\
\end{array}$ & $\mathrm{NR}$ & 4.87 & 2 & $<01$ & NR & $<10$ & NR & 12.3 & 3 \\
\hline 196 & 0270 & 3 & & & 0,030 & 3 & 0.332 & 4 & 40.9 & 0 \\
\hline 203 & & & 4.76 & 4 & & & $<2.5$ & NR & 10.2 & 1 \\
\hline 204 & & & & & 0,031 & 3 & & & & \\
\hline 209 & 0,240 & 4 & 4.74 & 4 & & & 0.340 & 4 & & \\
\hline 215 & 0640 & 0 & 4.90 & 2 & 0020 & 0 & 1.000 & 2 & 24.4 & 0 \\
\hline 221 & 0200 & 1 & 4.80 & 3 & 0.005 & 0 & & & & \\
\hline 224 & 0241 & 4 & 4.07 & 0 & 0,026 & 3 & 0.401 & 4 & 15.0 & 2 \\
\hline
\end{tabular}


Table 10. -Laboratory performance ratings for standard reference water sample Hg-20 (mercury)

(MPV, most probable value; ug/L, micrograms per liter, Lab, laboratory number, V/1 number of reported values of 1 value; RV, reported value; <, less than)

\begin{tabular}{|llll|}
\hline Rating & Absolute Z-value & Rating & Absolute Z-value \\
4 (Excellent) & $0.00-0.50$ & 1 (Questionable) & $1.51-2.00$ \\
3 (Good) & $0.51-1.00$ & $O$ (Poor) & greater than 2.00 \\
2 (Satisfactory) & $1.01-1.50$ & NR (Not Rated) & \\
\hline
\end{tabular}

2 (Satisfactory) $\quad 1.01-1.50$ $M P V=4,4, \mathrm{~g} h$

F-pseudosigma $=038$

\begin{tabular}{|c|c|c|c|}
\hline Lab & $V / 1$ & RV & Rating \\
\hline 1 & & 462 & 3 \\
\hline 3 & & 4.33 & 4 \\
\hline 11 & & 440 & 4 \\
\hline 13 & & 468 & 3 \\
\hline 15 & & 4,40 & 4 \\
\hline 16 & & 400 & 2 \\
\hline 18 & & 440 & 4 \\
\hline 24 & & 510 & 1 \\
\hline 32 & & 417 & 3 \\
\hline 34 & & 450 & 4 \\
\hline 36 & & 378 & 1 \\
\hline 39 & & 410 & 3 \\
\hline 42 & & 393 & 2 \\
\hline 45 & & 514 & 1 \\
\hline 46 & & 431 & 4 \\
\hline 48 & & 635 & 0 \\
\hline 50 & & 480 & 2 \\
\hline 52 & & 4,60 & 4 \\
\hline 55 & & 433 & 4 \\
\hline 58 & & 330 & 0 \\
\hline 69 & & 437 & 4 \\
\hline 70 & & 482 & 2 \\
\hline 75 & & 425 & 4 \\
\hline 76 & & 438 & 4 \\
\hline 81 & & 390 & 2 \\
\hline 86 & & 354 & 0 \\
\hline 87 & & 340 & 0 \\
\hline 89 & & 450 & 4 \\
\hline 92 & & 476 & 3 \\
\hline 96 & & 442 & 4 \\
\hline 97 & & 477 & 3 \\
\hline 100 & & 4.47 & 4 \\
\hline 105 & & 4.35 & 4 \\
\hline 108 & & 483 & 2 \\
\hline 109 & & 402 & 2 \\
\hline 111 & & 412 & 3 \\
\hline 114 & & 100 & 0 \\
\hline 118 & & 460 & 4 \\
\hline 127 & & 487 & 2 \\
\hline 128 & & 450 & 4 \\
\hline 133 & & 470 & 3 \\
\hline 134 & & 462 & 3 \\
\hline 138 & & 3.90 & 2 \\
\hline 141 & & 455 & 4 \\
\hline 142 & & 440 & 4 \\
\hline 145 & & 460 & 4 \\
\hline 146 & & 4.45 & 4 \\
\hline 149 & & 470 & 3 \\
\hline 180 & & 578 & 0 \\
\hline 182 & & 550 & 0 \\
\hline 194 & & 450 & 4 \\
\hline 198 & & 370 & 1 \\
\hline 203 & & 425 & 4 \\
\hline 213 & & 460 & 4 \\
\hline 215 & & 600 & 0 \\
\hline 219 & & 390 & 2 \\
\hline 221 & & 410 & 3 \\
\hline 231 & & 440 & 4 \\
\hline 234 & & 4.43 & 4 \\
\hline 235 & & 600 & 0 \\
\hline 241 & & 426 & 4 \\
\hline
\end{tabular}


Table 11. -Laboratory performance ratings for standard reference sediment sample SED-5 (bed material)

\section{--Continued}

(MPV,most probable value; ug/g, micrograms per gram; $\mathrm{mg} / \mathrm{g}$, milligrams per gram; Lab, laboratory number; OLR, overall laboratory rating for all reported values;

V/26, number of reported values of 26 possible values; RV, reported value; <, less than)
Rating Absolute Z-value Rating Absolute Z-value

$\begin{array}{llll}4 \text { (Excellent) } & 0.00-0.50 & 1 \text { (Questionable) } & 1.51-2.00 \\ 3 \text { (Good) } & 0.51-1.00 & O \text { (Poor) } & \text { greater than }\end{array}$

2 (Satisfactory) $\quad 1.01-1.50 \quad$ NR (Not Rated)

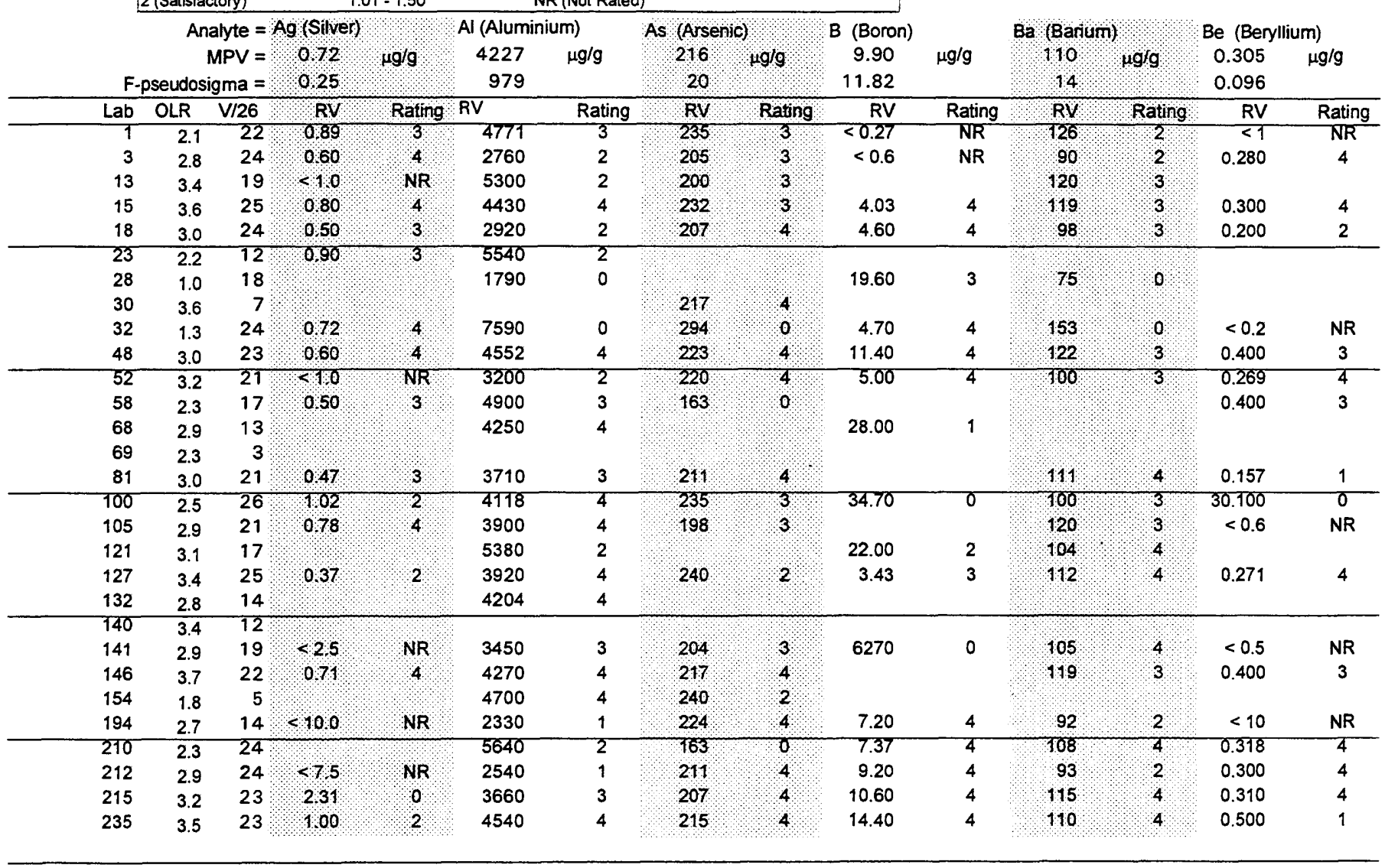


Table 11. -Laboratory performance ratings for standard reference sediment sample SED-5 (bed material)

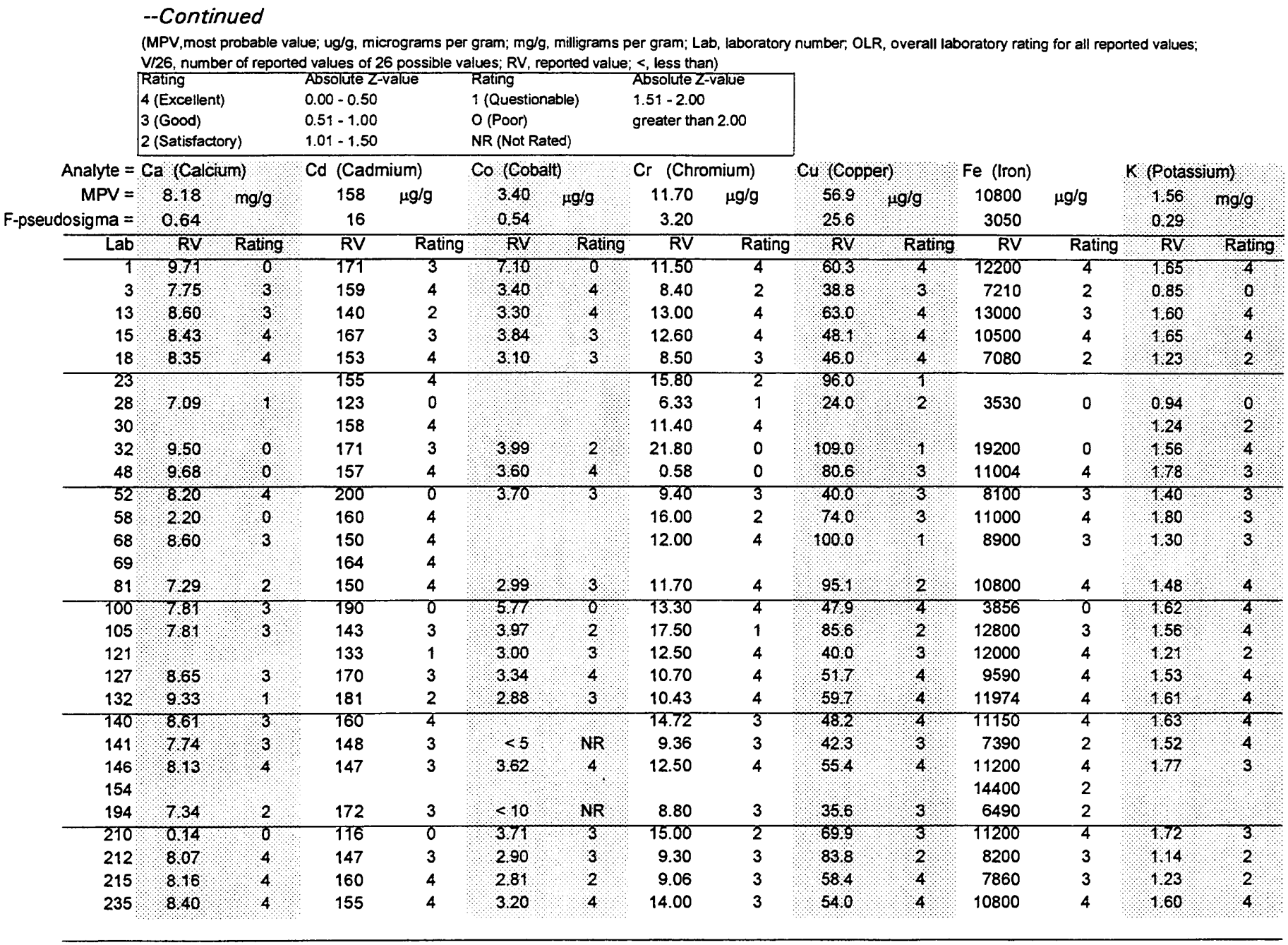


Table 11. -Laboratory performance ratings for standard reference sediment sample SED-5 (bed material) --Continued

(MPV,most probable vaiue; ug/g, micrograms per gram; $\mathrm{mg} / \mathrm{g}$, milligrams per gram; Lab, laboratory number; OLR, overall laboratory rating for all reported values; VI26, number of reported values of 26 possible values; $R V$, reported value; <, less than)

\begin{tabular}{|llll}
\hline Rating & Absolute Z-value & Rating & Absolute Z-value \\
4 (Excellent) & $0.00-0.50$ & 1 (Questionable) & $1.51-2.00$ \\
3 (Good) & $0.51-1.00$ & $O$ (Poor) & greater than 2.00 \\
2 (Satisfactory) & $1.01-1.50$ & NR (Not Rated) & \\
\hline
\end{tabular}

\begin{tabular}{|c|c|c|c|c|c|c|c|c|c|c|c|c|c|c|}
\hline & 2 (Satisfac & & $1.01-1.50$ & & NR (Not R & & & & & & & & & \\
\hline Analyte $=$ & Ef ( Wthit & & $\mathrm{Mg}$ (Mag & esium) & $\mathrm{Mn}$ lMar & nese) & Mo (Moly & lenum) & $\mathrm{Na}$ (Sod & & Ni (Nick & & $\mathrm{Pb}$ (Lead) & \\
\hline MPV $=$ & 382 & $\mu g / 9$ & 1.91 & $\mathrm{mg} / \mathrm{g}$ & 257 & $\mu g / g$ & 1.01 & $\mu g / g$ & 0290 & $g / g$ & 6.68 & $\mu \mathrm{g} / \mathrm{g}$ & 309 & $\mu \mathrm{g} / \mathrm{g}$ \\
\hline F-pseudosigma = & 0,98 & & 0.24 & & 19 & & 0.19 & & 0031 & & 1.69 & & 18 & \\
\hline Lab & RV & Rating & RV & Rating & $\mathrm{RV}$ & Rating & RV & Rating & $\mathrm{RV}$ & Rating & $R V$ & Rating & RV & Rating \\
\hline 1 & 530 & म & 1.96 & 4 & 285 & 1? & $<1.4$ & NR & 0350 & 13 & 10.00 & 1 & 273 & 3 \\
\hline 3 & 380 & 4 & 1.52 & 1 & 224 & 1 & $<0.6$ & NR & 0,290 & 4 & 5.50 & 3 & 299 & 3 \\
\hline 13 & & & 1.90 & 4 & 270 & 3 & & & 0,300 & 4 & 6.20 & 4 & 320 & 3 \\
\hline 15 & 446 & 3 & 2.16 & 2 & 268 & 3 & 1.06 & 4 & 0,307 & 3 & 6.61 & 4 & 311 & 4 \\
\hline 18 & & & 1.62 & 2 & 246 & 3 & 1.20 & 3 & 0.261 & 3 & 4.80 & 2 & 327 & 3 \\
\hline 23 & & & 0.94 & 0 & 292 & 1 & $<6$ & NR & 0133 & 0 & 6.74 & 4 & 319 & 3 \\
\hline 28 & & & 1.41 & 1 & 190 & 0 & $<0.5$ & NR & 0.290 & 4 & 8.83 & 2 & 216 & 0 \\
\hline 30 & & & & & & & & & & & 8.21 & 3 & 303 & 4 \\
\hline 32 & 570 & 1 & 3.20 & 0 & 322 & 0 & 634 & 0 & 0,380 & 0 & 9.40 & 1 & 325 & 3 \\
\hline 48 & & & 2.34 & 1 & 257 & 4 & 1.02 & 4 & 0,592 & 0 & 6.48 & 4 & 317 & 4 \\
\hline 52 & & & 1.80 & 4 & 250 & 4 & $<2.2$ & NR & 0.263 & 3 & 5.10 & 3 & 310 & 4 \\
\hline 58 & & & 52.00 & 0 & 309 & 0 & & & 0.280 & 4 & 8.00 & 3 & 300 & 4 \\
\hline 68 & & & 1.80 & 4 & 260 & 4 & $<3.7$ & NR & & & 8.70 & 2 & 340 & 1 \\
\hline 69 & & & & & & & & & & & & & 322 & 3 \\
\hline 81 & & & 1.85 & 4 & 245 & 3 & $<0.692$ & NR & 0.246 & 2 & 8.50 & 2 & 263 & 0 \\
\hline 100 & 331 & 3 & 1.92 & 4 & 266 & 4 & 0.74 & 2 & 0,350 & 1 & 6.62 & 4 & 314 & 4 \\
\hline 105 & & & 1.93 & 4 & 251 & 4 & 1.25 & 2 & 0.228 & 1 & 8.28 & 3 & 308 & 4 \\
\hline 121 & & & 1.96 & 4 & 245 & 3 & & & 02290 & 4 & 6.00 & 4 & & \\
\hline 127 & 382 & 4 & 1.97 & 4 & 273 & 3 & $<2$ & NR & 0283 & 4 & 6.09 & 4 & 284 & 2 \\
\hline 132 & & & 2.74 & 0 & 295 & 0 & & & 0285 & 4 & 8.28 & 3 & & \\
\hline 140 & & & 2.10 & 3 & 257 & 4 & & & $0 / 294$ & 4 & 5.01 & 3 & 327 & 2 \\
\hline 141 & & & 1.83 & 4 & 238 & 2 & $<5$ & NR & $0: 250$ & 2 & 7.26 & 4 & 294 & 3 \\
\hline 146 & & & 2.05 & 3 & 259 & 4 & 0.94 & 4 & 0,313 & 3 & 6.48 & 4 & 305 & 4 \\
\hline 154 & & & & & & & & & & & & & 345 & 1 \\
\hline 194 & $<1000$ & & 1.50 & 1 & 253 & 4 & $<50$ & NR & $<1$ & NR & $<10$ & NR & 331 & 2 \\
\hline 210 & & & 1.77 & 3 & 237 & 2 & 1.00 & 4 & 0276 & 4 & 5.53 & 3 & 239 & 0 \\
\hline 212 & 200 & 1 & 1.47 & 1 & 249 & 4 & $<10$ & NR & 0,290 & 4 & 7.20 & 4 & 307 & 4 \\
\hline 215 & & & 1.87 & 4 & 243 & 3 & 0.88 & 3 & 0,260 & 3 & 5.31 & 3 & 309 & 4 \\
\hline 235 & & & 2.10 & 3 & 270 & 3 & 1.00 & 4 & & & 6.80 & 4 & 290 & 2 \\
\hline
\end{tabular}


Table 11. Laboratory performance ratings for standard reference sediment sample SED-5 (bed material)

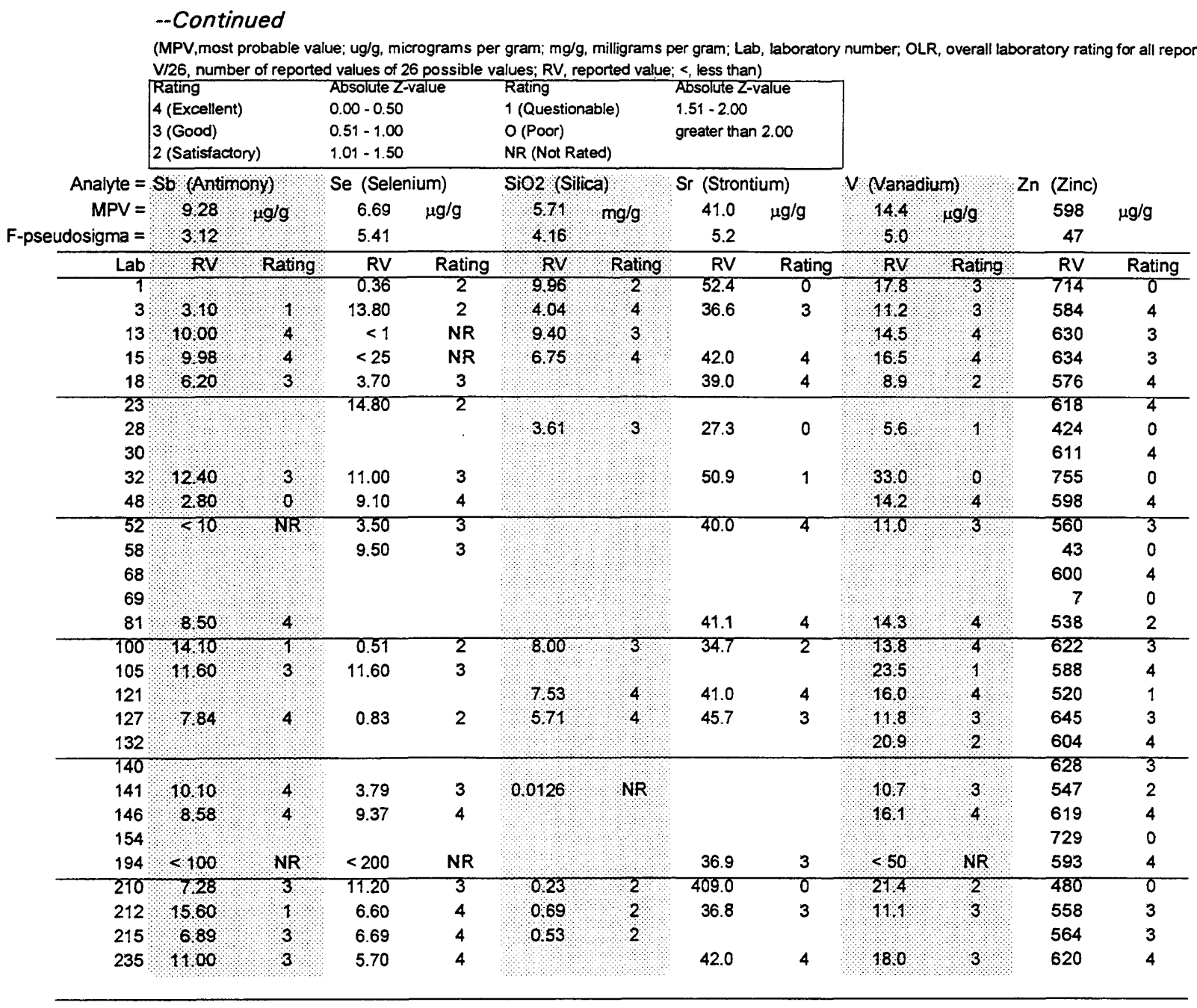


Table 12. Statistical summary of reported data for standard reference water sample $T-135$ (trace constituents)

Definition of analytical methods, abbreviations, and symbols

Analytical methods
$\begin{array}{ll}\text { 0. Other/Not reported } & \\ \text { 1. AA: direct, air } & =\text { atomic absorption: direct,air } \\ \text { 2. AA: direct, N2O } & =\text { atomic absorption: direct, nitrous oxide } \\ \text { 3. AA: graphite furnace } & =\text { atomic absorption: graphite furnace } \\ \text { 4. ICP } & =\text { inductively coupled plasma } \\ \text { 5. DCP } & =\text { direct current plasma } \\ \text { 6. ICPMS } & =\text { inductively coupled plasma/mass spectrometry } \\ \text { 10. AA: extraction } & =\text { atomic absorption: extraction [chelating agent(s) specified] } \\ \text { 11. AA: hydride } & =\text { atomic absorption: hydride [reducing agent specified] } \\ \text { 12. AA: flame emission } & =\text { atomic absorption: flame emission } \\ \text { 22. Color: } & =\text { colorimetric [color reagent specified] }\end{array}$

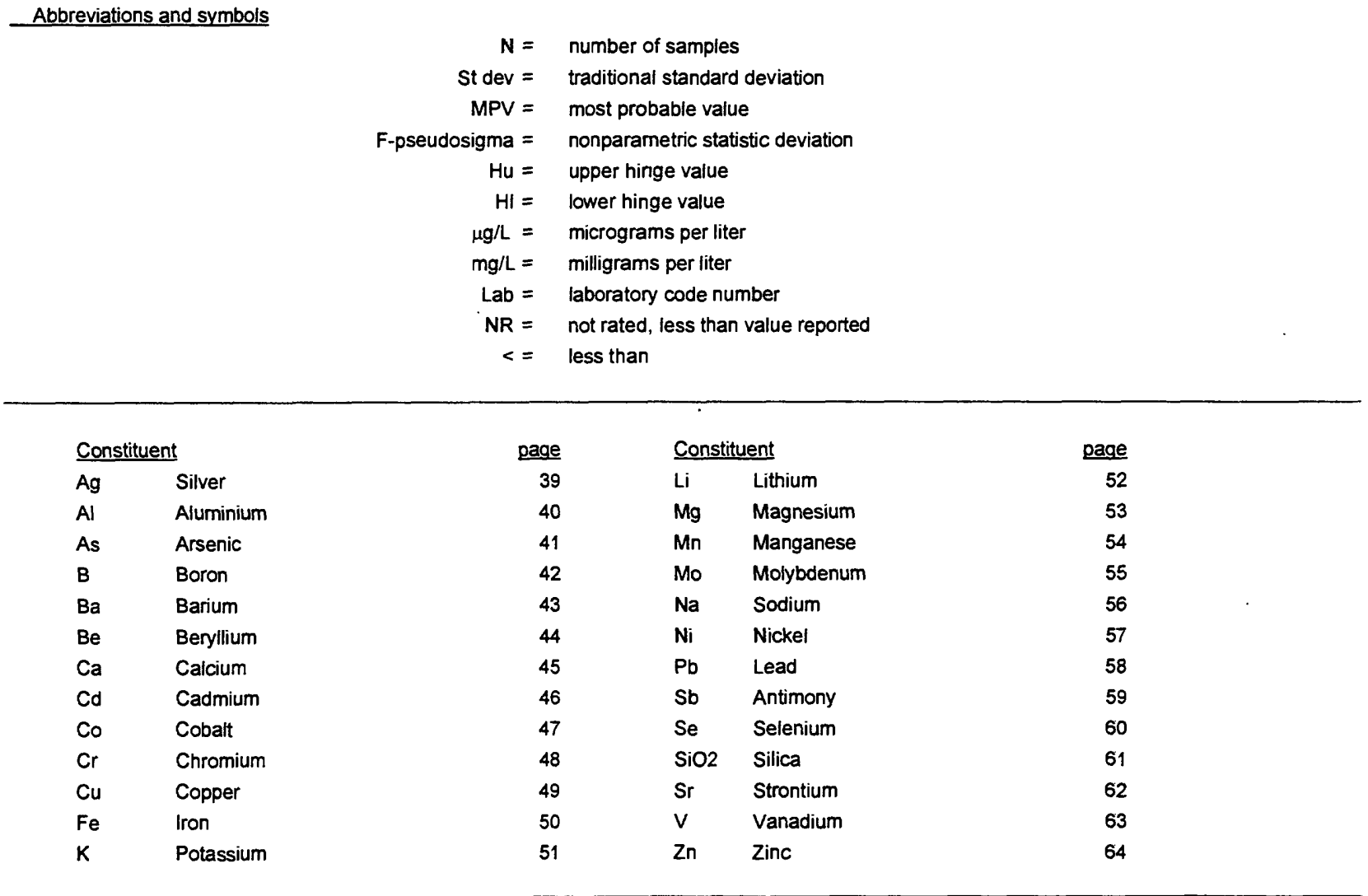


Table 12. Statistical summary of reported data for standard reference water sample T-135 (trace constituents)--Continued Ag (Silver) $\mu \mathrm{g} / \mathrm{l}$

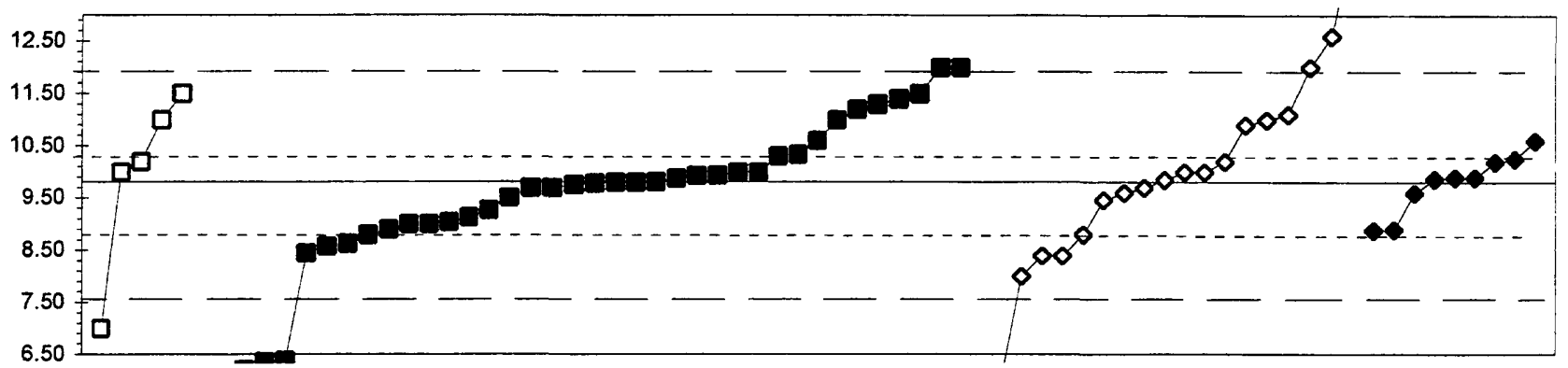

$-\square-1-3 \multimap-\infty-6$

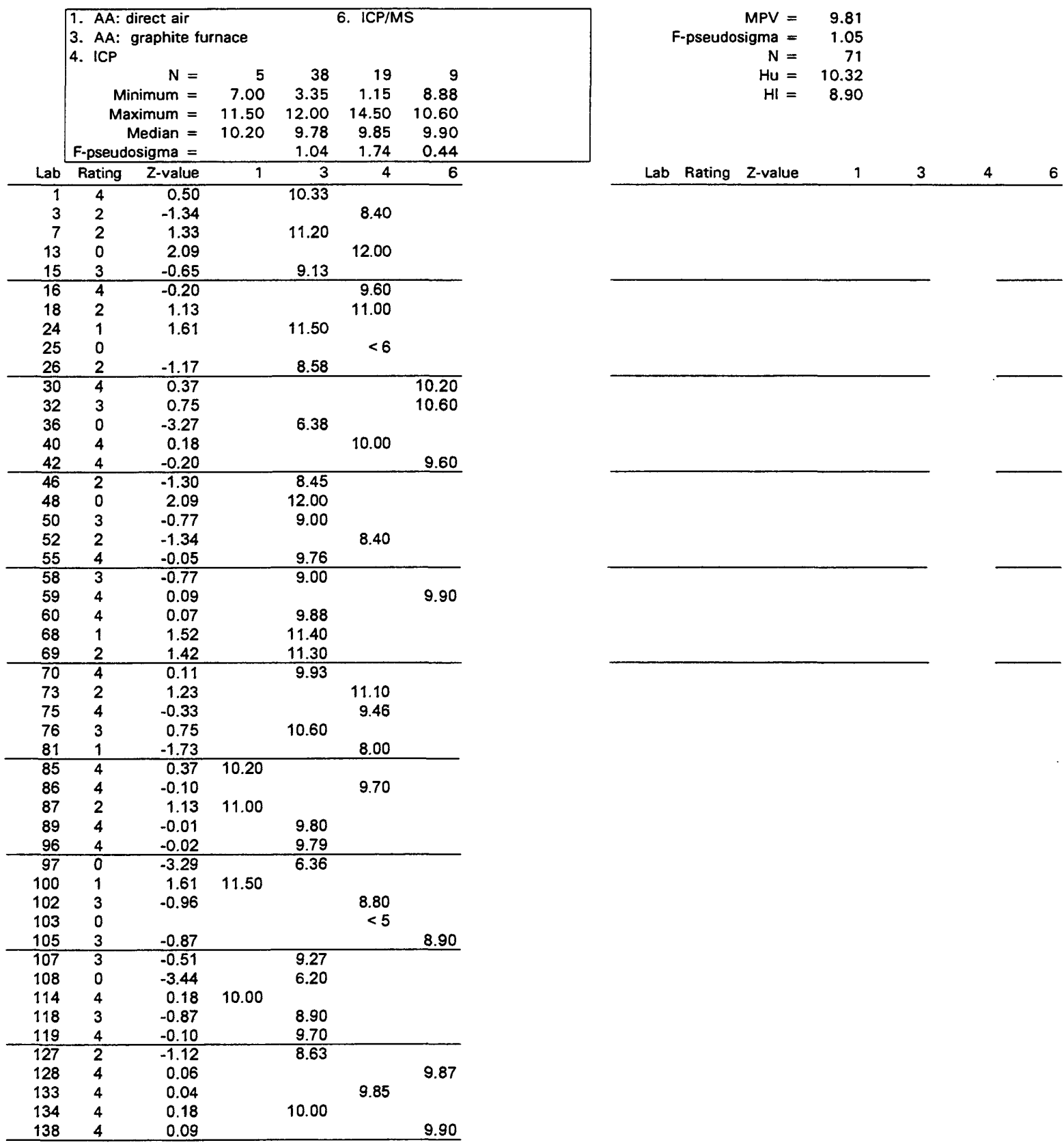


Table 12. Statistical summary of reported data for standard reference water sample T-135 (trace constituents)--Continued Al (Aluminum) $\mu \mathrm{g} / \mathrm{l}$

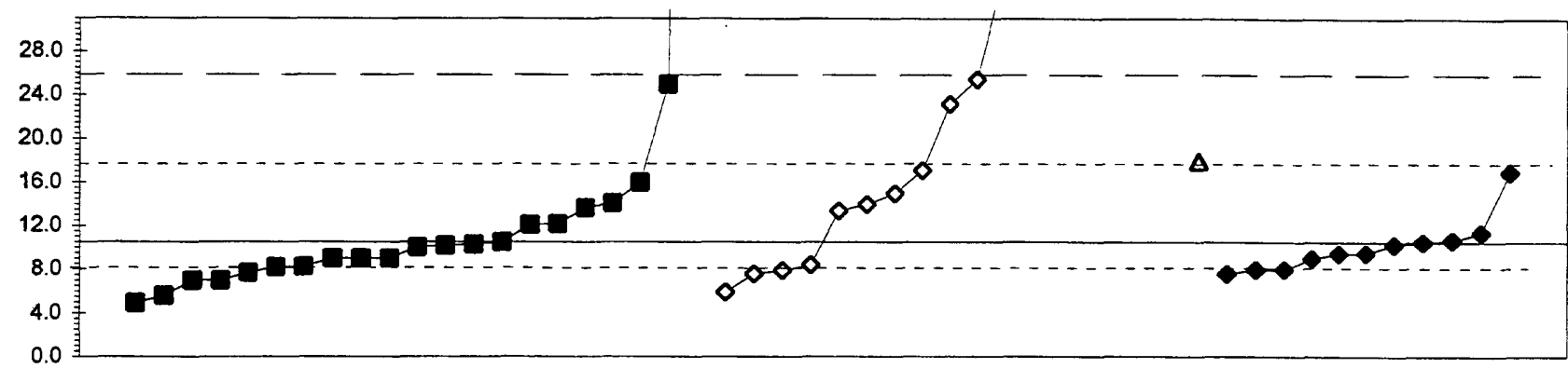

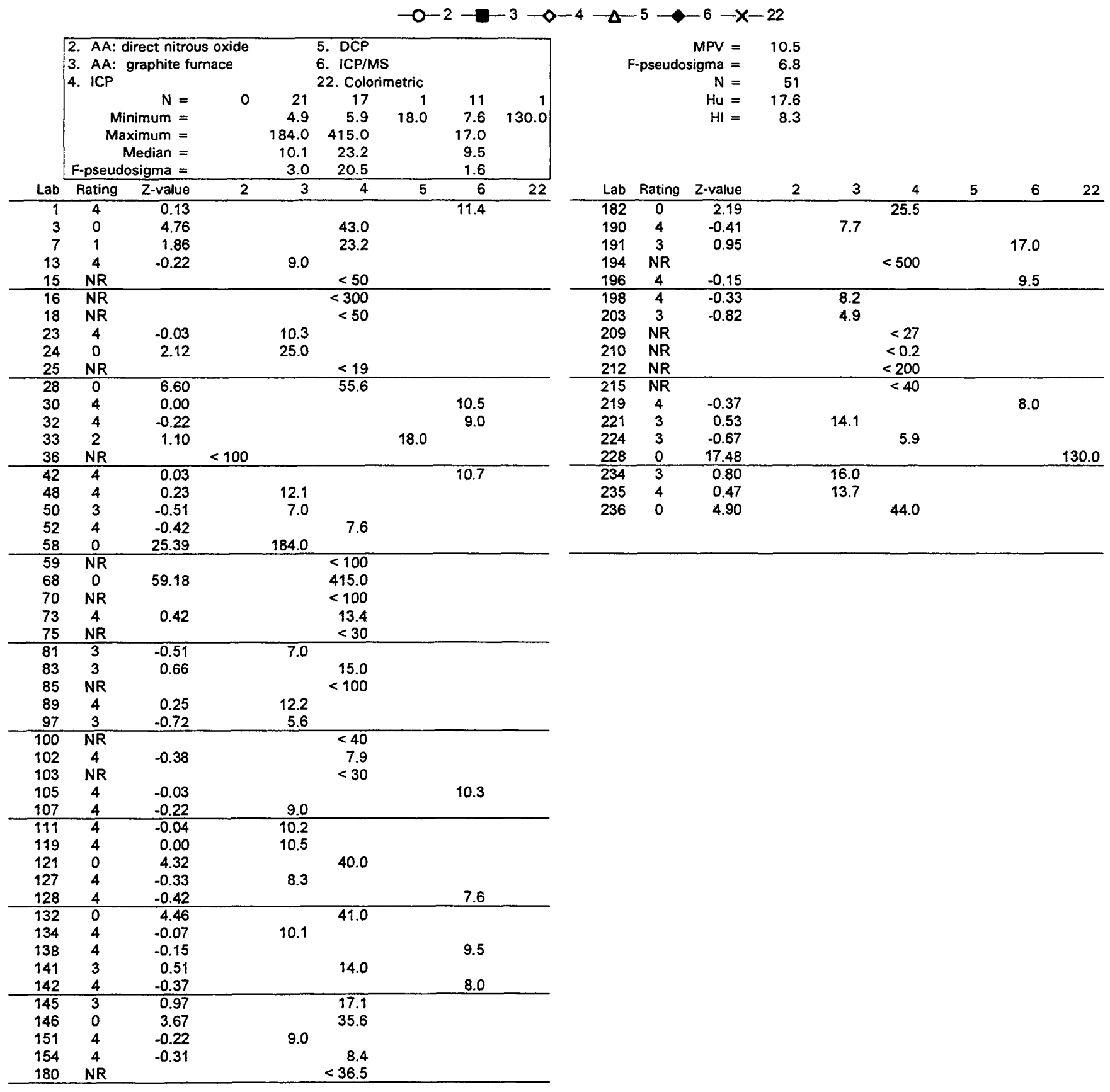


Table 12. Statistical summary of reported data for standard reference water sample T-135 (trace constituents)--Continued As (Arsenic) $\mu \mathrm{g} / \mathrm{l}$

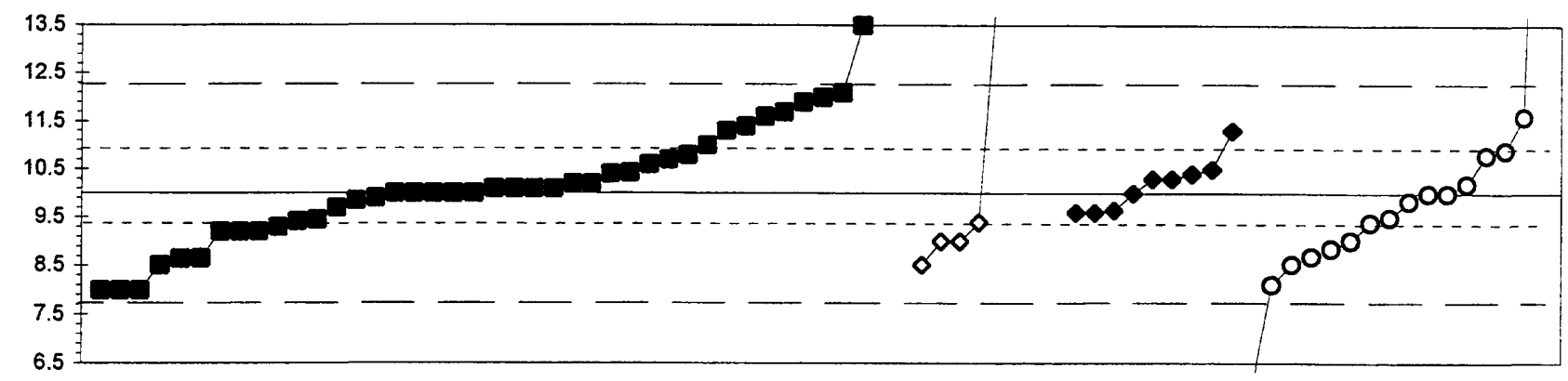

\begin{tabular}{|c|c|c|c|c|c|c|c|c|c|c|c|c|c|}
\hline \multirow[b]{2}{*}{ Lab } & $\begin{array}{lll}\text { 3. } & \text { AA: } \\
\text { 4. } & \text { ICP } \\
\text { 6. } & \text { ICP/I } \\
& & \\
& & \\
\text { F-pseud }\end{array}$ & $\begin{aligned} \text { raphite fu } \\
\mathrm{S} \\
\mathrm{N}= \\
\text { imum }= \\
\text { imum }= \\
\text { ledian }= \\
\text { sigma }=\end{aligned}$ & $\begin{array}{r}\text { lace } \\
42 \\
8.0 \\
14.7 \\
10.1 \\
1.2 \\
\end{array}$ & $\begin{array}{r}8 \\
8.5 \\
48.0 \\
12.1 \\
9.1 \\
\end{array}$ & $\begin{array}{r}9 \\
9.6 \\
11.3 \\
10.3 \\
0.6 \\
\end{array}$ & $\begin{array}{r}16 \\
6.0 \\
24.0 \\
9.7 \\
1.3\end{array}$ & & F-pseudo & $\begin{aligned} \text { MPV } & = \\
\text { sigma } & = \\
N & = \\
H u & = \\
H I & =\end{aligned}$ & $\begin{array}{r}10.0 \\
1.1 \\
75 \\
10.9 \\
9.4\end{array}$ & & & \\
\hline & Rating & $Z$-value & 3 & 4 & 6 & 11 & Lab & Rating & $Z$-value & 3 & 4 & 6 & 11 \\
\hline 1 & 4 & 0.00 & 10.0 & & & & 128 & 4 & -0.36 & & & 9.6 & \\
\hline $\begin{array}{l}3 \\
7\end{array}$ & $\begin{array}{l}4 \\
4\end{array}$ & $\begin{array}{l}0.00 \\
0.00\end{array}$ & $\begin{array}{l}10.0 \\
10.0\end{array}$ & & & & $\begin{array}{l}133 \\
134\end{array}$ & $\begin{array}{l}4 \\
4\end{array}$ & $\begin{array}{l}0.09 \\
0.00\end{array}$ & 10.1 & & & 10.0 \\
\hline 13 & 3 & -0.72 & 9.2 & & & & 138 & 3 & -0.72 & 9.2 & & & \\
\hline 15 & 2 & -1.33 & 8.5 & & & & 141 & NR & & & $<50$ & & \\
\hline 16 & NR & & $<60$ & & & & 142 & 4 & -0.32 & & & 9.6 & \\
\hline 18 & 2 & 1.44 & & & & 11.6 & 145 & 0 & 4.23 & & 14.7 & & \\
\hline 24 & 0 & 3.15 & 13.5 & & & & 146 & 2 & -1.33 & & 8.5 & & \\
\hline 25 & NR & & & $<50$ & & & 151 & 2 & -1.17 & & & & 8.7 \\
\hline 26 & 3 & -0.87 & & & & 9.0 & 154 & 4 & 0.09 & 10.1 & & & \\
\hline 28 & 3 & -0.90 & & 9.0 & & & 180 & NR & & & $<33.4$ & & \\
\hline 30 & 2 & 1.17 & & & 11.3 & & 182 & 2 & -1.31 & & & & 8.5 \\
\hline 32 & 4 & 0.27 & & & 10.3 & & 190 & 4 & 0.00 & 10.0 & & & \\
\hline 34 & 3 & 0.72 & 10.8 & & & & 193 & 1 & -1.80 & 8.0 & & & \\
\hline 35 & 4 & -0.14 & & & & 9.8 & 194 & 4 & 0.00 & 10.0 & & & \\
\hline 36 & 2 & -1.21 & 8.7 & & & & 196 & 4 & 0.45 & & & 10.5 & \\
\hline 39 & 3 & -0.54 & & & & 9.4 & 198 & 2 & 1.44 & 11.6 & & & \\
\hline 42 & 4 & 0.27 & & & 10.3 & & 203 & 3 & -0.63 & 9.3 & & & \\
\hline 45 & 4 & -0.49 & 9.5 & & & & 210 & 0 & 9.44 & & 20.5 & & \\
\hline 46 & 4 & -0.27 & 9.7 & & & & 212 & 4 & -0.09 & 9.9 & & & \\
\hline 48 & 3 & 0.63 & 10.7 & & & & 213 & 1 & 1.53 & 11.7 & & & \\
\hline 50 & 0 & -3.60 & & & & 6.0 & 215 & 0 & 3.60 & 14.0 & & & \\
\hline 52 & 3 & -0.90 & & 9.0 & & & 219 & 4 & -0.36 & & & 9.6 & \\
\hline 55 & 1 & 1.71 & 11.9 & & & & 221 & 4 & -0.13 & 9.9 & & & \\
\hline 58 & 0 & 12.59 & & & & 24.0 & 224 & 3 & -0.54 & & 9.4 & & \\
\hline 59 & 4 & 0.00 & & & 10.0 & & 231 & 3 & 0.54 & 10.6 & & & \\
\hline 60 & $i$ & 1.89 & 12.1 & & & & 234 & 4 & 0.18 & 10.2 & & & \\
\hline 68 & 1 & -1.80 & 8.0 & & & & 235 & 2 & 1.17 & 11.3 & & & \\
\hline 69 & 4 & 0.18 & 10.2 & & & & 236 & 0 & 34.17 & & 48.0 & & \\
\hline 70 & 3 & -0.52 & 9.4 & & & & 241 & 3 & -0.72 & 9.2 & & & \\
\hline 75 & 2 & -1.03 & & & & 8.9 & & & & & & & \\
\hline 76 & 2 & 1.26 & 11.4 & & & & & & & & & & \\
\hline 80 & 3 & 0.90 & 11.0 & & & & & & & & & & \\
\hline 81 & 1 & 1.80 & 12.0 & & & & & & & & & & \\
\hline 85 & 4 & -0.45 & & & & 9.5 & & & & & & & \\
\hline 86 & 1 & -1.69 & & & & 8.1 & & & & & & & \\
\hline 87 & 4 & 0.18 & & & & 10.2 & & & & & & & \\
\hline 89 & 3 & 0.72 & & & & 10.8 & & & & & & & \\
\hline 96 & 4 & 0.09 & 10.1 & & & & & & & & & & \\
\hline 97 & 4 & 0.09 & 10.1 & & & & & & & & & & \\
\hline 100 & 2 & -1.21 & 8.7 & & & & & & & & & & \\
\hline 102 & 0 & 10.79 & & 22.0 & & & & & & & & & \\
\hline 103 & NR & & & $<10$ & & & & & & & & & \\
\hline 105 & 4 & 0.36 & & & 10.4 & & & & & & & & \\
\hline 108 & 1 & -1.80 & 8.0 & & & & & & & & & & \\
\hline 109 & 4 & 0.39 & 10.4 & & & & & & & & & & \\
\hline 118 & 0 & 4.23 & 14.7 & & & & & & & & & & \\
\hline 119 & 4 & 0.00 & & & & 10.0 & & & & & & & \\
\hline 126 & 3 & 0.81 & & & & 10.9 & & & & & & & \\
\hline 127 & 4 & 0.36 & 10.4 & & & & & & & & & & \\
\hline
\end{tabular}


Table 12. Statistical summary of reported data for standard reference water sample T-135 (trace constituents)--Continued B (Boron) $\mu \mathrm{g} / \mathrm{l}$

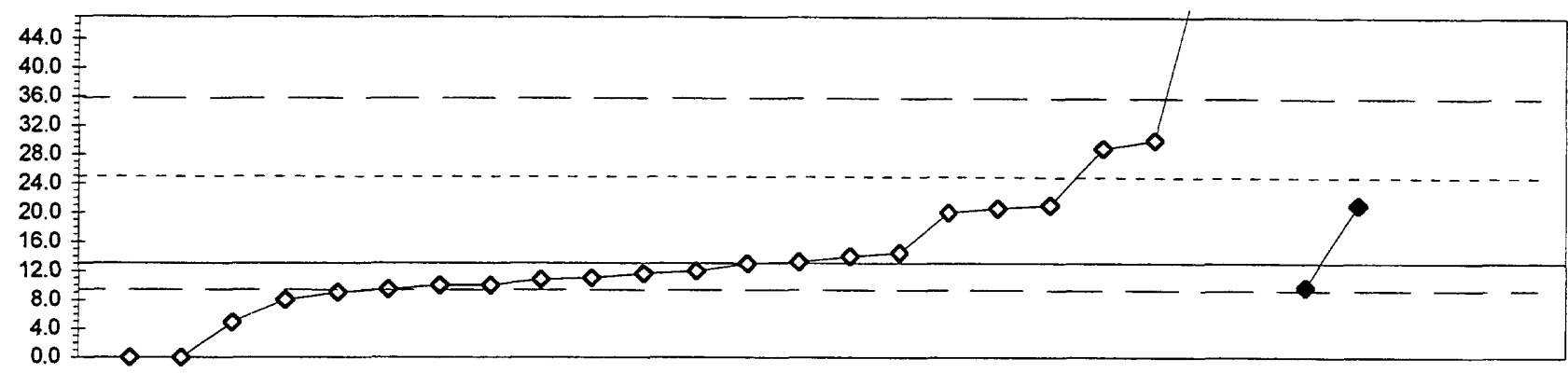

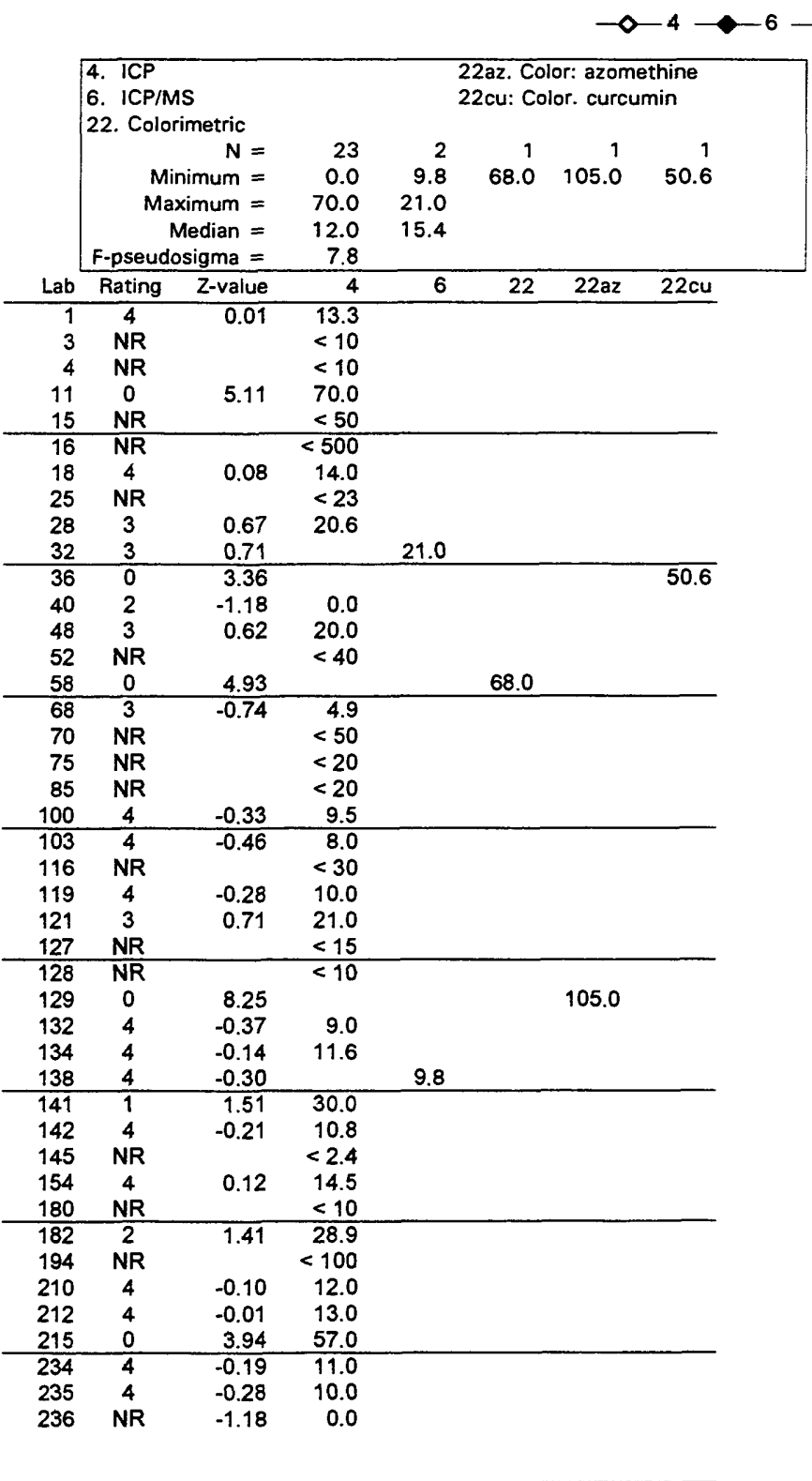


Table 12. Statistical summary of reported data for standard reference water sample T-135 (trace constituents)--Continued $\mathrm{Ba}$ (Barium) $\mu \mathrm{g} / \mathrm{l}$

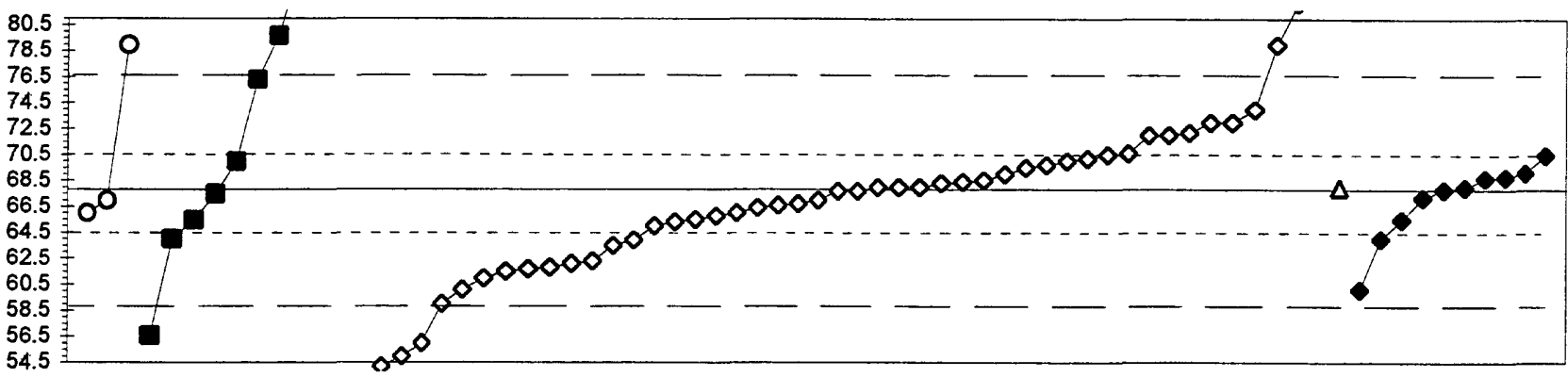

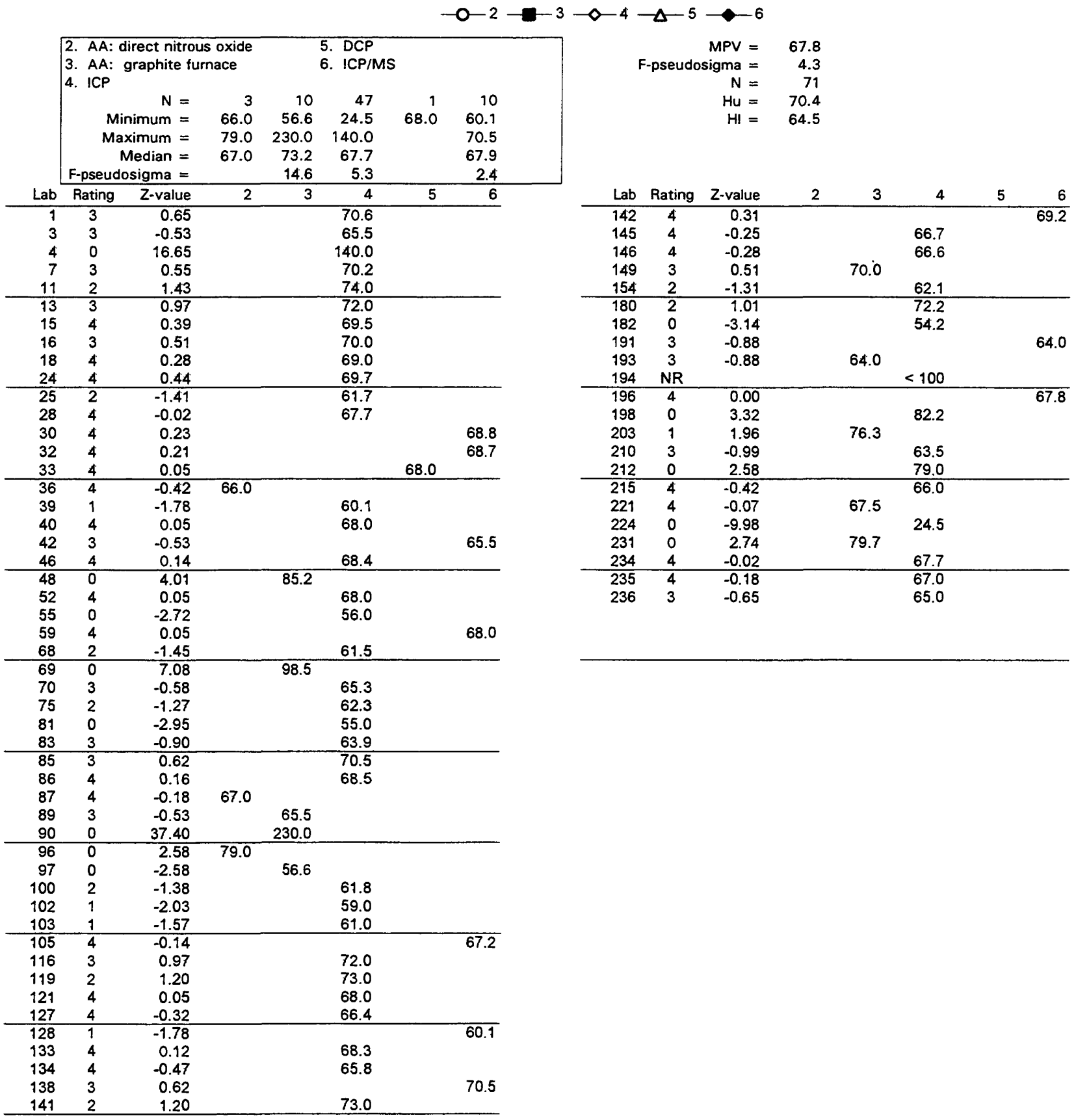


Table 12. Statistical summary of reported data for standard reference water sample T-135 (trace constituents)--Continued $\mathrm{Be}$ (Berylium) $\mu \mathrm{g} / \mathrm{l}$

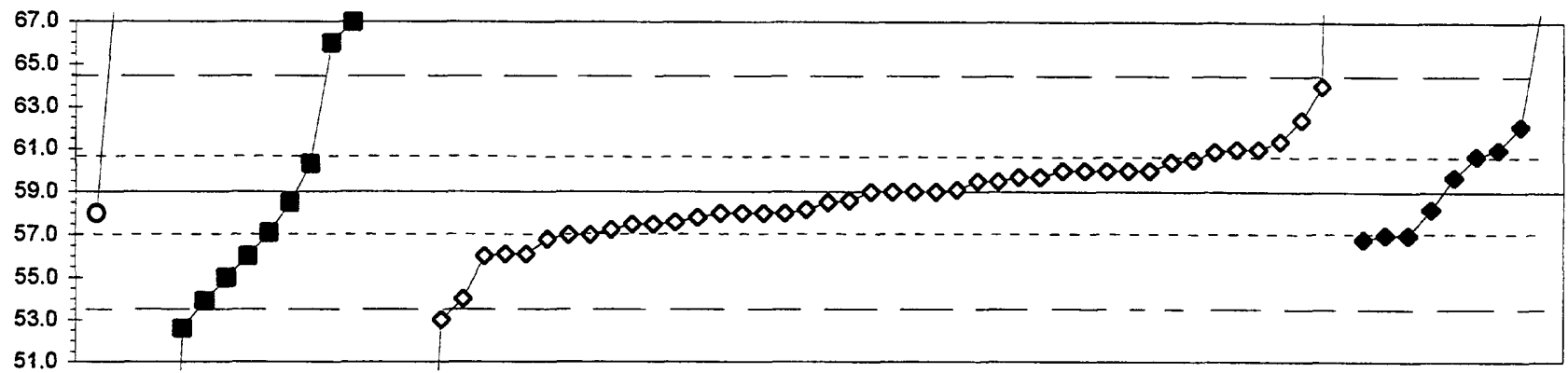

$-0-2 \rightarrow-3-4 \multimap 6$

\begin{tabular}{|c|c|c|c|c|c|c|}
\hline & \multicolumn{3}{|c|}{$\begin{array}{l}\text { 2. AA: direct nitrous oxide } \\
\text { 3. AA: graphite furnace } \\
\text { 4. ICP }\end{array}$} & \multicolumn{3}{|c|}{ 6. ICP/MS } \\
\hline & \multirow{2}{*}{\multicolumn{2}{|c|}{$\begin{aligned} N & = \\
\text { Minimum } & =\end{aligned}$}} & 3 & 12 & 44 & 9 \\
\hline & & & 58.0 & 22.0 & 31.0 & 56.8 \\
\hline & \multicolumn{2}{|c|}{ Maximum $=$} & 78.8 & 675.0 & 119.0 & 68.0 \\
\hline & & ledian $=$ & 70.4 & 57.8 & 59.0 & 59.7 \\
\hline & \multicolumn{2}{|c|}{ F-pseudosigma $=$} & & 8.9 & 1.9 & 3.0 \\
\hline Lab & Rating & $Z$-value & 2 & 3 & 4 & 6 \\
\hline 1 & 3 & -0.67 & & & 57.2 & \\
\hline 3 & 3 & -0.76 & & & 57.0 & \\
\hline 4 & 0 & 22.80 & & & 119.0 & \\
\hline 7 & 3 & -0.57 & & & 57.5 & \\
\hline 11 & 4 & 0.38 & & & 60.0 & \\
\hline 13 & 4 & 0.38 & & & 60.0 & \\
\hline 15 & 4 & 0.27 & & & 59.7 & \\
\hline 16 & 4 & -0.38 & & & 58.0 & \\
\hline 18 & 4 & 0.00 & & & 59.0 & \\
\hline 24 & 0 & 2.66 & & 66.0 & & \\
\hline 25 & 4 & 0.19 & & & 59.5 & \\
\hline 28 & 0 & 4.33 & 70.4 & & & \\
\hline 30 & 2 & 1.18 & & & & 62.1 \\
\hline 32 & 0 & 3.42 & & & & 68.0 \\
\hline 36 & 0 & 7.52 & 78.8 & & & \\
\hline 39 & 4 & -0.46 & & & 57.8 & \\
\hline 40 & 4 & 0.38 & & & 60.0 & \\
\hline 42 & 4 & 0.27 & & & & 59.7 \\
\hline 46 & 3 & 0.91 & & & 61.4 & \\
\hline 48 & 4 & 0.38 & & & 60.0 & \\
\hline$\frac{70}{50}$ & $\frac{7}{1}$ & -1.52 & & 55.0 & & \\
\hline 52 & 4 & -0.38 & & & 58.0 & \\
\hline 55 & 3 & 0.53 & & & 60.4 & \\
\hline 58 & 2 & -1.14 & & 56.0 & & \\
\hline 59 & 3 & 0.76 & & & & 61.0 \\
\hline 60 & 3 & -0.72 & & 57.1 & & \\
\hline 68 & 0 & -2.28 & & & 53.0 & \\
\hline 69 & 4 & 0.49 & & 60.3 & & \\
\hline 70 & 4 & -0.38 & & & 58.0 & \\
\hline 75 & 3 & -0.84 & & & 56.8 & \\
\hline 76 & 3 & 0.65 & & & & 60.7 \\
\hline 81 & 1 & -1.90 & & & 54.0 & \\
\hline 83 & 2 & -1.10 & & & 56.1 & \\
\hline 85 & 4 & 0.00 & & & 59.0 & \\
\hline 86 & 4 & -0.15 & & & 58.6 & \\
\hline 89 & 0 & 4.10 & & 69.8 & & \\
\hline 97 & 0 & -2.43 & & 52.6 & & \\
\hline 100 & 3 & 0.57 & & & 60.5 & \\
\hline 102 & 4 & 0.00 & & & 59.0 & \\
\hline 103 & 3 & -0.76 & & & 57.0 & \\
\hline 105 & 3 & -0.76 & & & & 57.0 \\
\hline 114 & 4 & -0.38 & 58.0 & & & \\
\hline 119 & 1 & -1.94 & & 53.9 & & \\
\hline 127 & 3 & -0.57 & & & 57.5 & \\
\hline 128 & 4 & -0.30 & & & & 58.2 \\
\hline 133 & 1 & 1.90 & & & 64.0 & \\
\hline 134 & 3 & -0.54 & & & 57.6 & \\
\hline 138 & 4 & 0.00 & & & 59.0 & \\
\hline 141 & 3 & 0.76 & & & 61.0 & \\
\hline 142 & 4 & -0.32 & & & 58.2 & \\
\hline
\end{tabular}

\begin{tabular}{|c|c|c|c|c|c|}
\hline Lab & Rating & $Z$-value & 2 & 4 & 6 \\
\hline 145 & 4 & 0.04 & & 59.1 & \\
\hline 146 & 4 & -0.19 & & 58.5 & \\
\hline 154 & 4 & 0.38 & & 60.0 & \\
\hline 180 & 3 & 0.72 & & 60.9 & \\
\hline 182 & 0 & -10.63 & & 31.0 & \\
\hline 194 & 0 & 234.08 & 675.0 & & \\
\hline 196 & 3 & -0.84 & & & 56.8 \\
\hline 198 & 2 & 1.29 & & 62.4 & \\
\hline 210 & 4 & 0.19 & & 59.5 & \\
\hline 212 & 3 & 0.76 & & 61.0 & \\
\hline 213 & 0 & 3.04 & 67.0 & & \\
\hline 215 & 2 & -1.14 & & 56.0 & \\
\hline 219 & 3 & -0.76 & & & 57.0 \\
\hline 224 & 2 & -1.10 & & 56.1 & \\
\hline 234 & 4 & 0.27 & & 59.7 & \\
\hline 235 & 4 & -0.19 & 58.5 & & \\
\hline 236 & 4 & -0.38 & & 58.0 & \\
\hline 241 & 0 & -14.06 & 22.0 & & \\
\hline
\end{tabular}


Table 12. Statistical summary of reported data for standard reference water sample T-135 (trace constituents)--Continued Ca (Calcium) $\mathrm{mg} / \mathrm{l}$

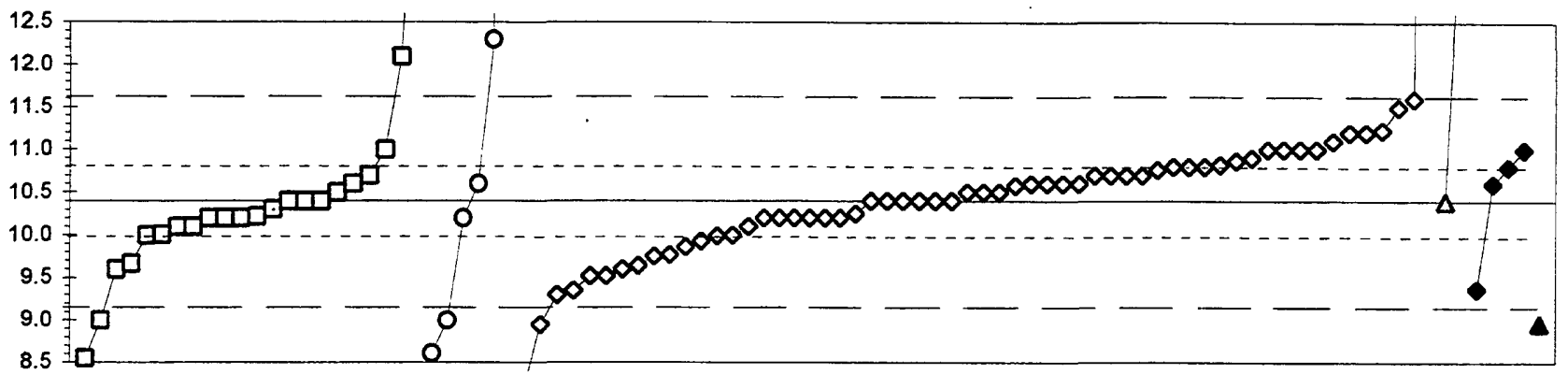

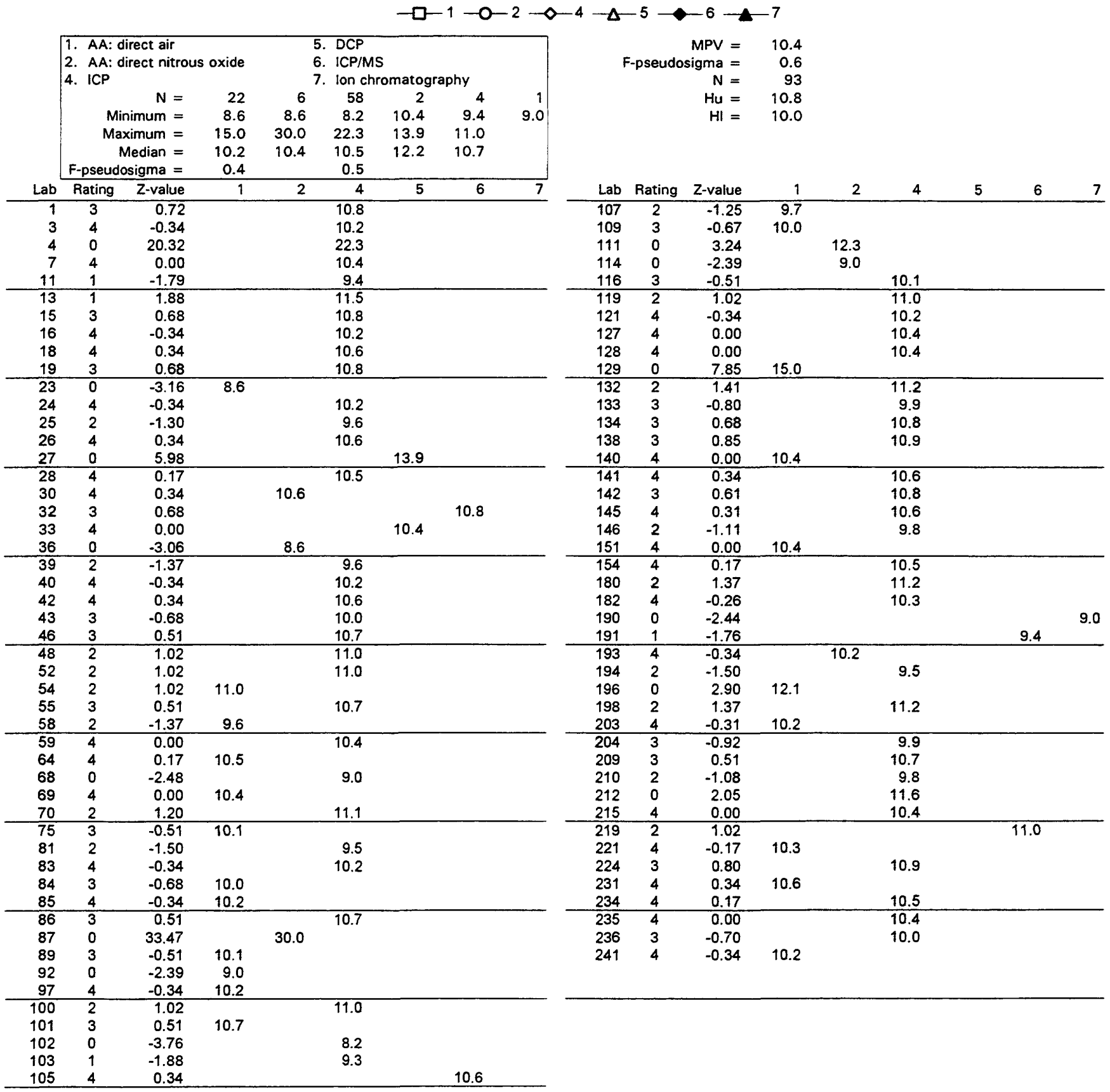


Table 12. Statistical summary of reported data for standard reference water sample T-135 (trace constituents)--Continued Cd (Cadmium) $\mu \mathrm{g} / \mathrm{l}$

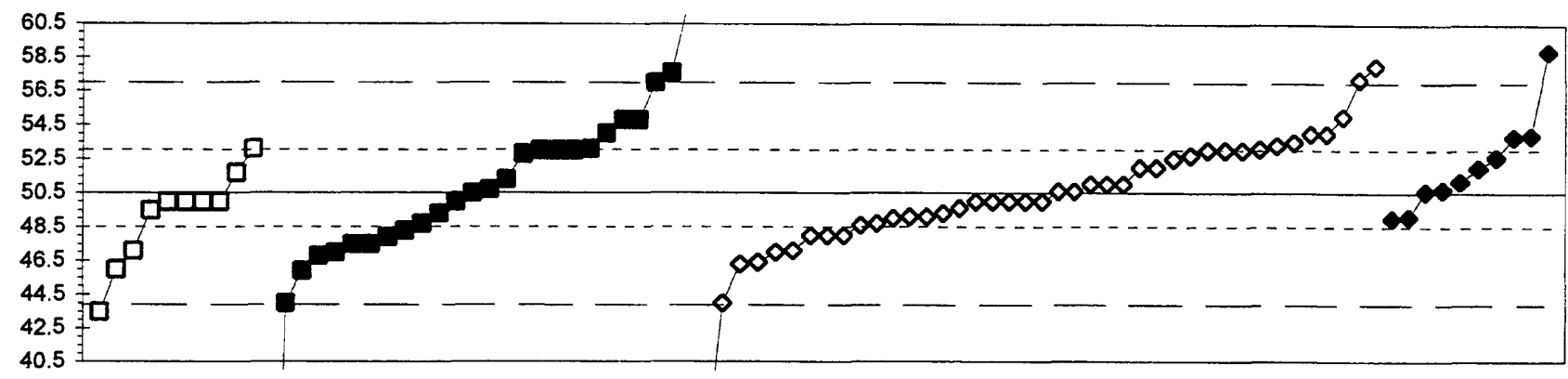

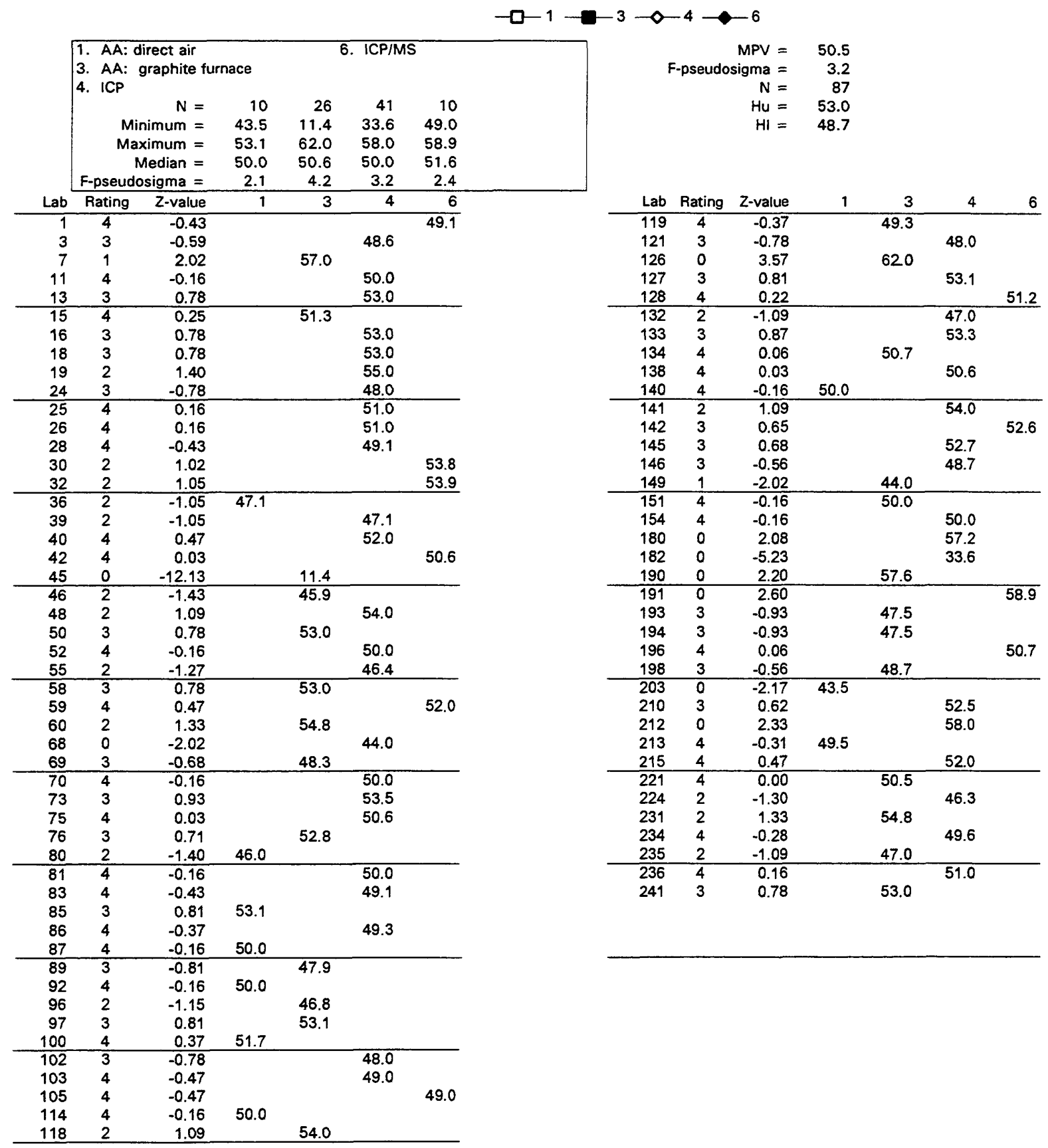


Table 12. Statistical summary of reported data for standard reference water sample T-135 (trace constituents)--Continued Co (Cobalt) $\mu \mathrm{g} / \mathrm{l}$
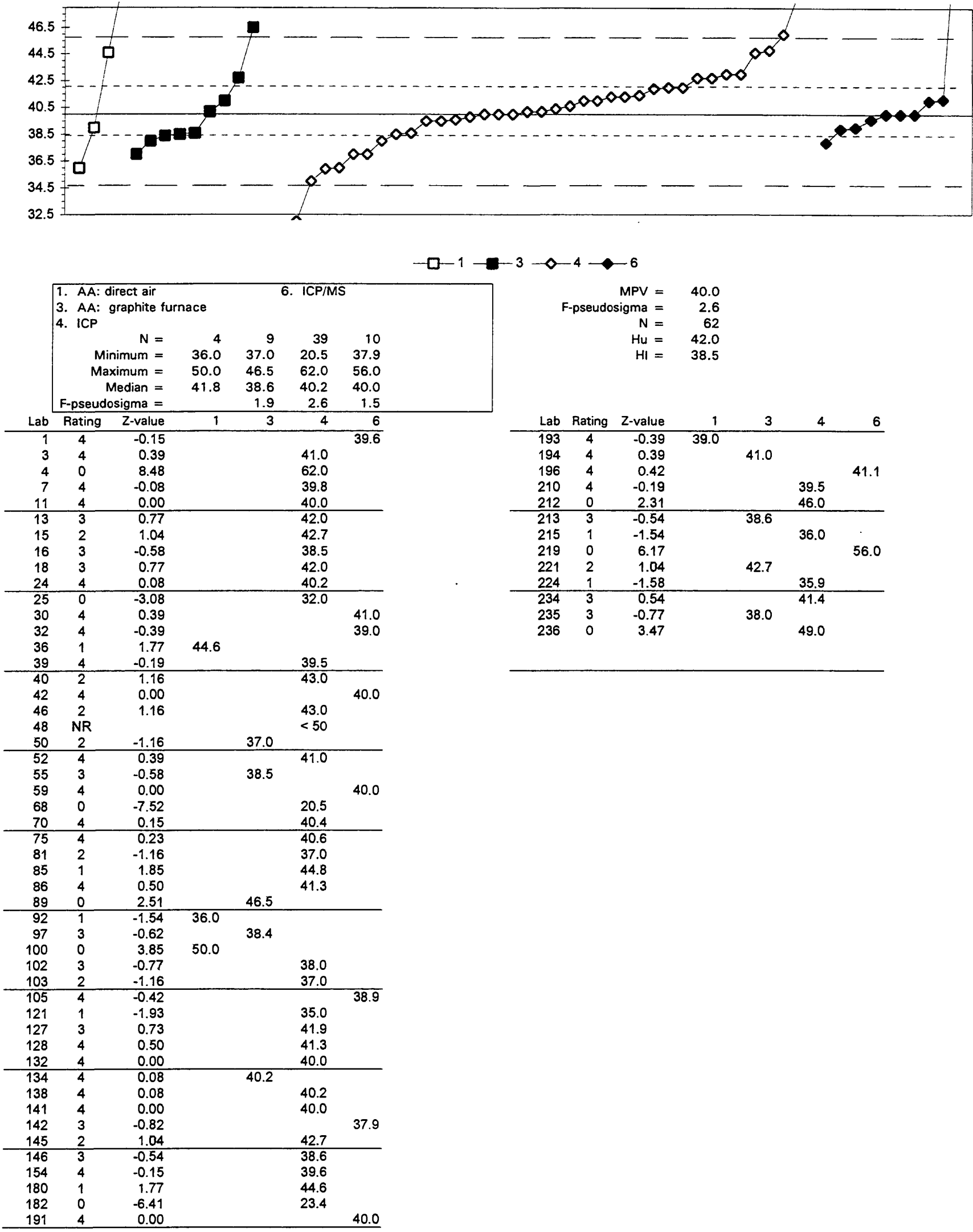
Table 12. Statistical summary of reported data for standard reference water sample T-135 (trace constituents)--Continued $\mathrm{Cr}$ (Chromium) $\mu \mathrm{g} / \mathrm{l}$

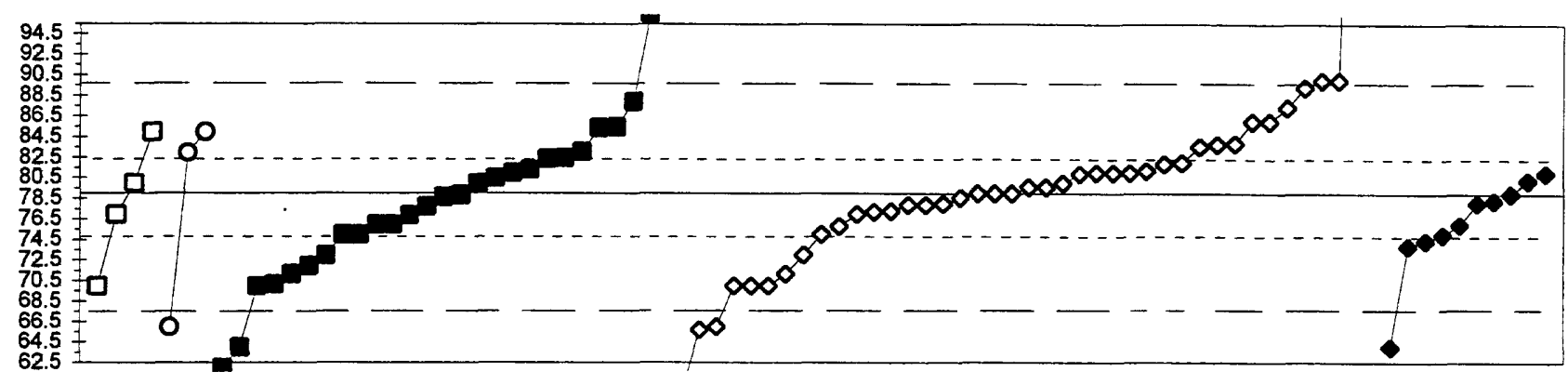

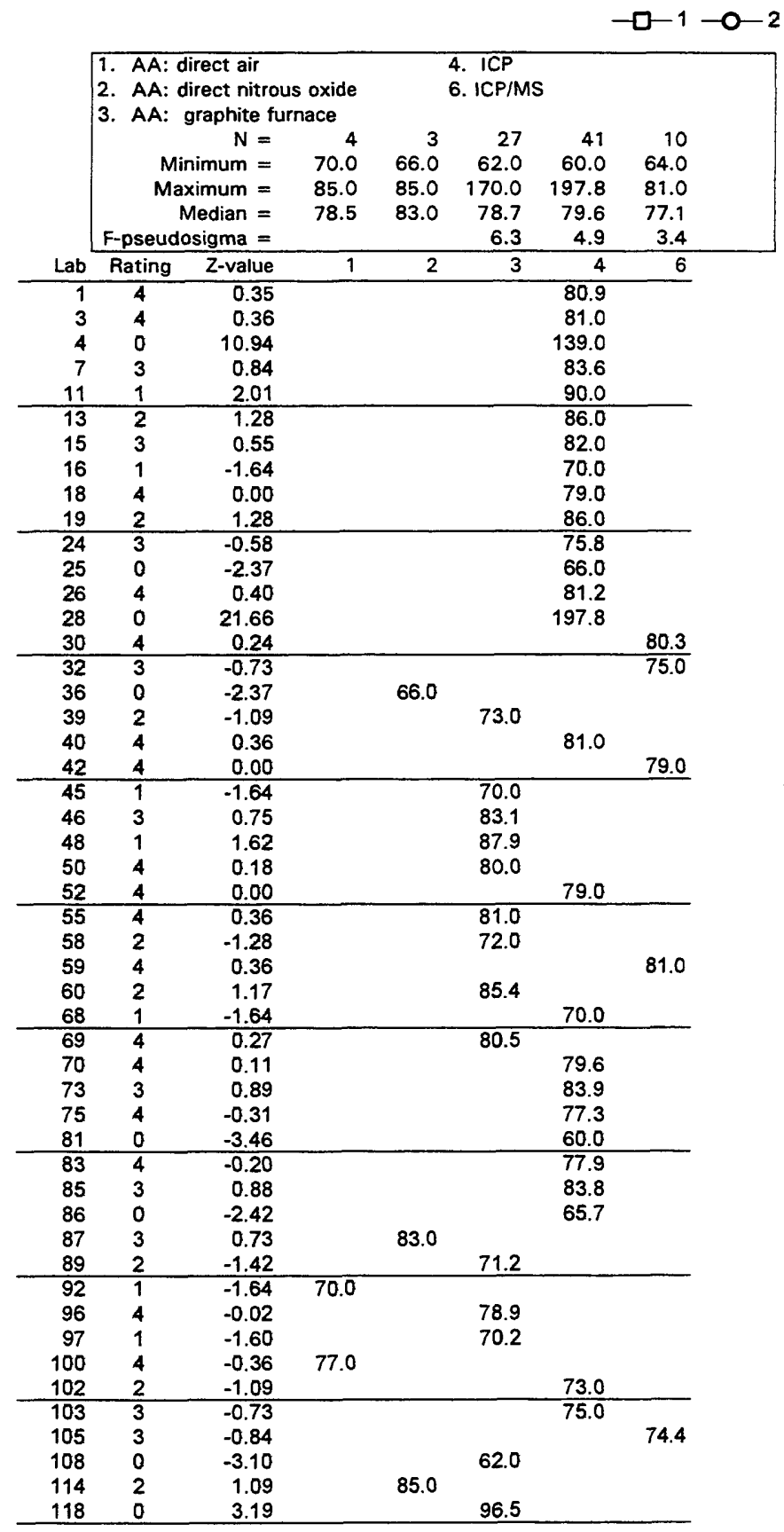

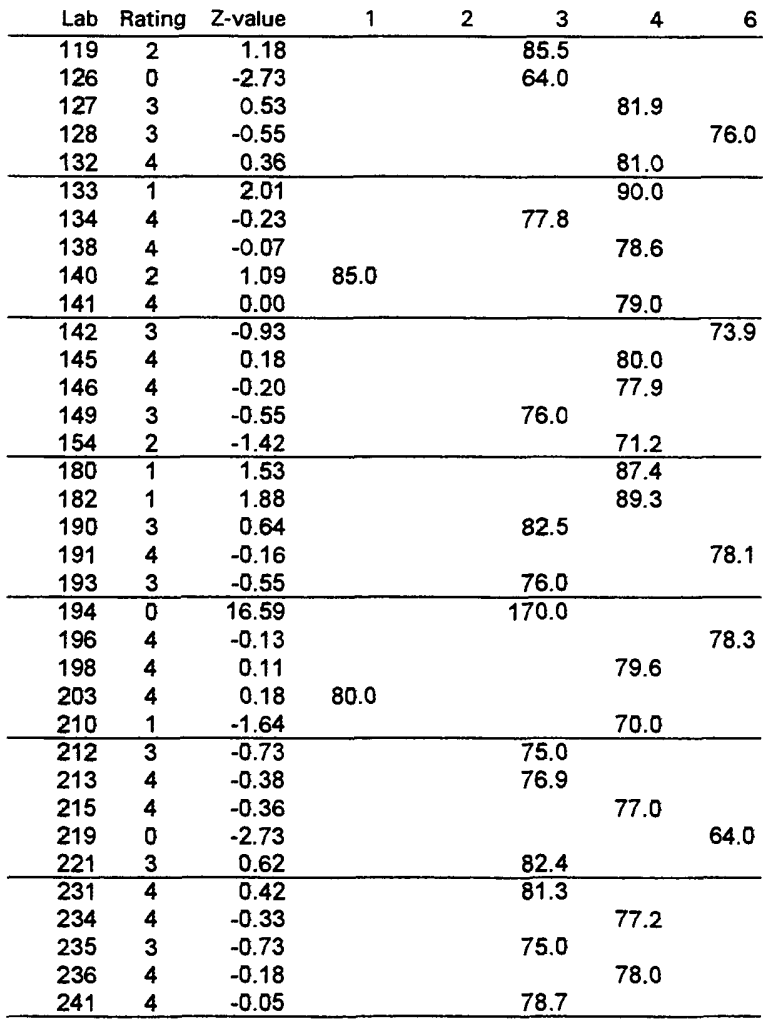


Table 12. Statistical summary of reported data for standard reference water sample T-135 (trace constituents)--Continued $\mathrm{Cu}$ (Copper) $\mu \mathrm{g} / \mathrm{l}$

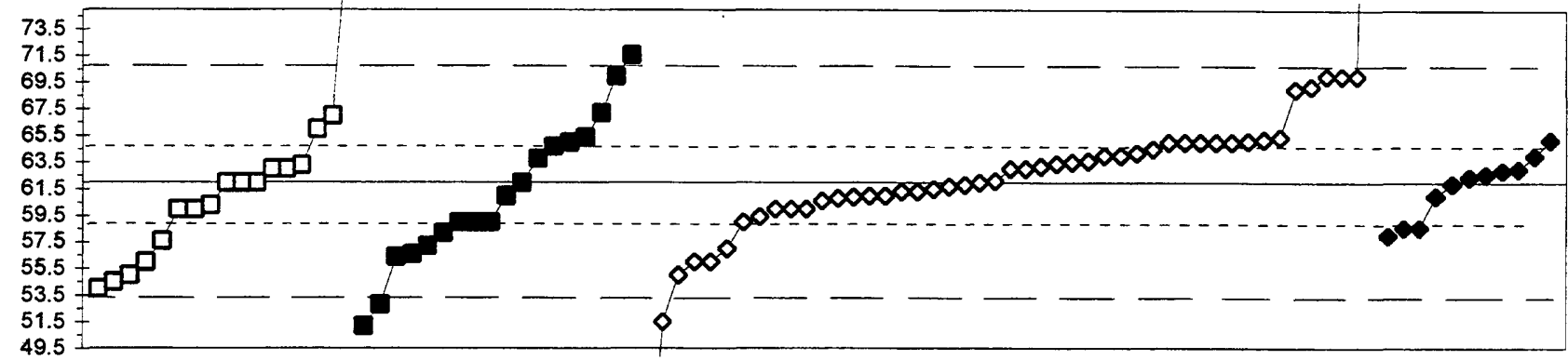

$-\square-1-3-0-4 \multimap 6$

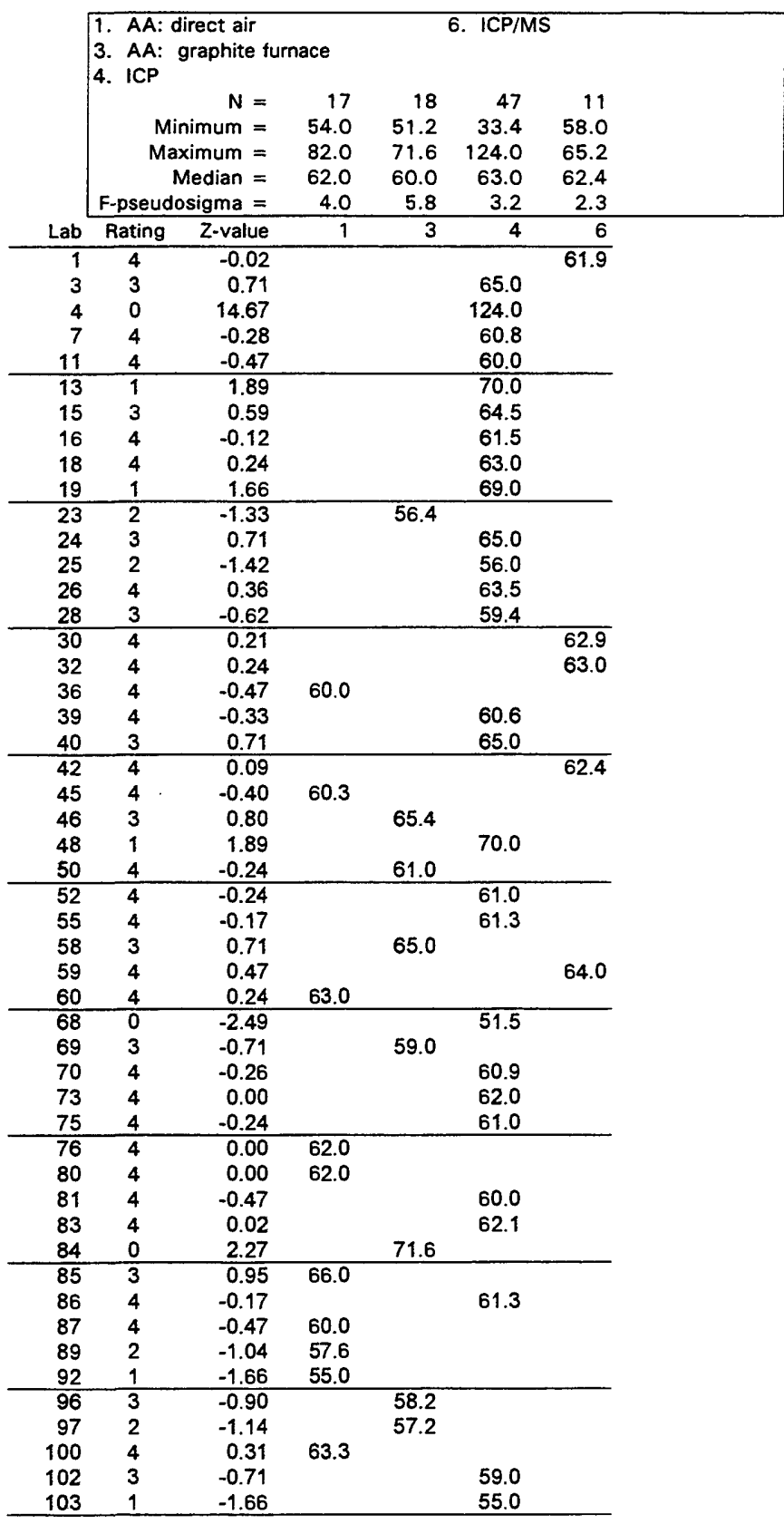

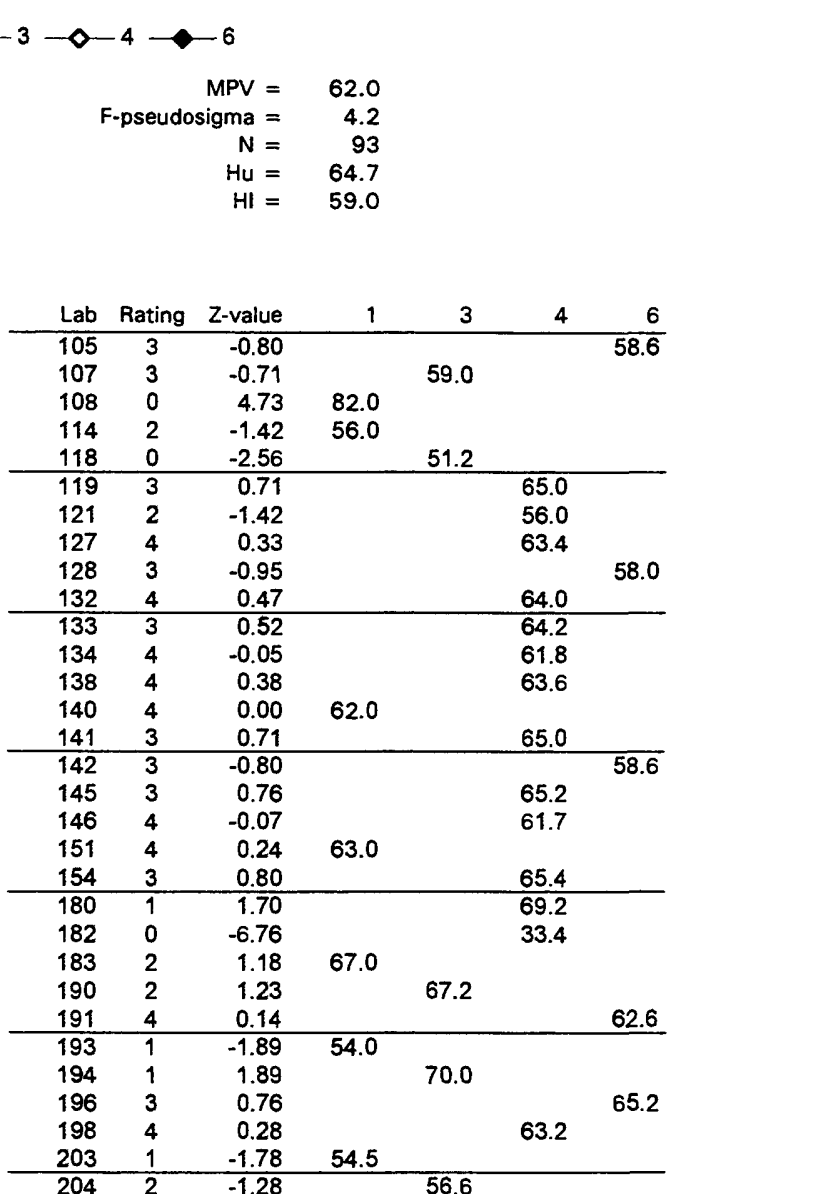

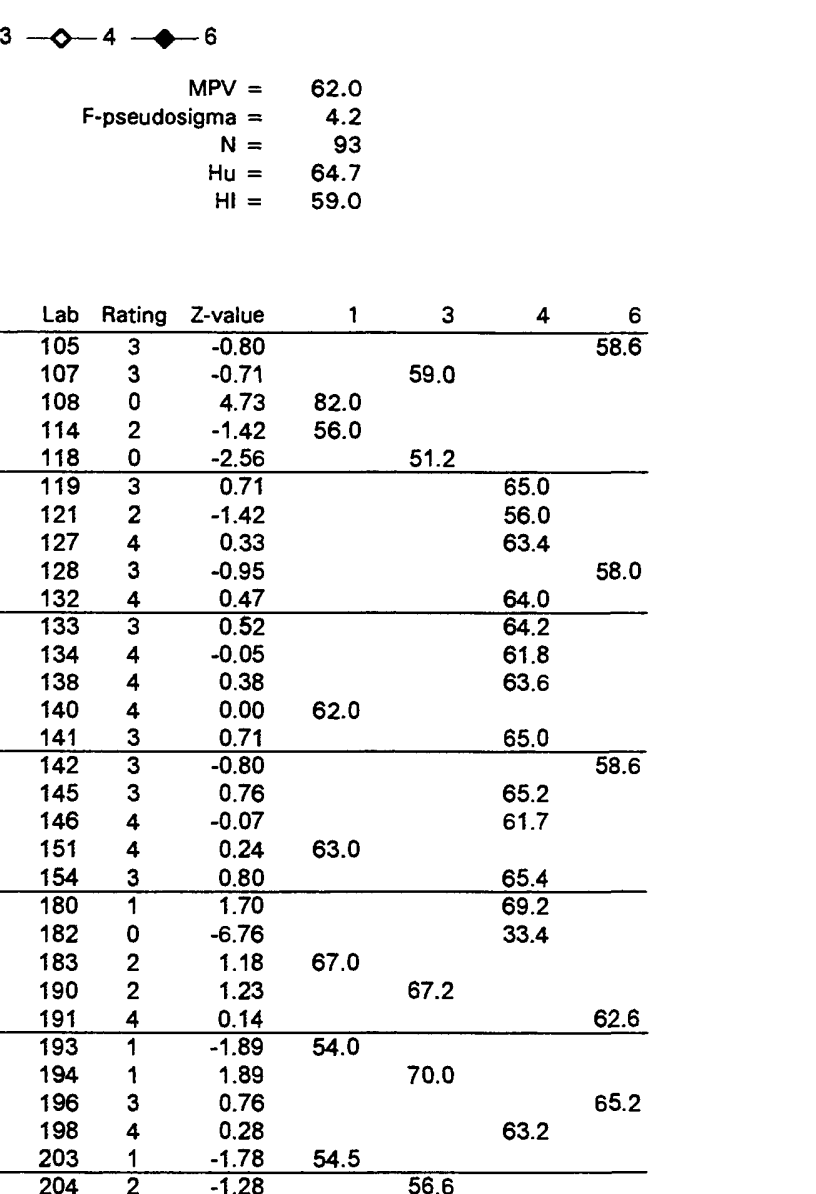

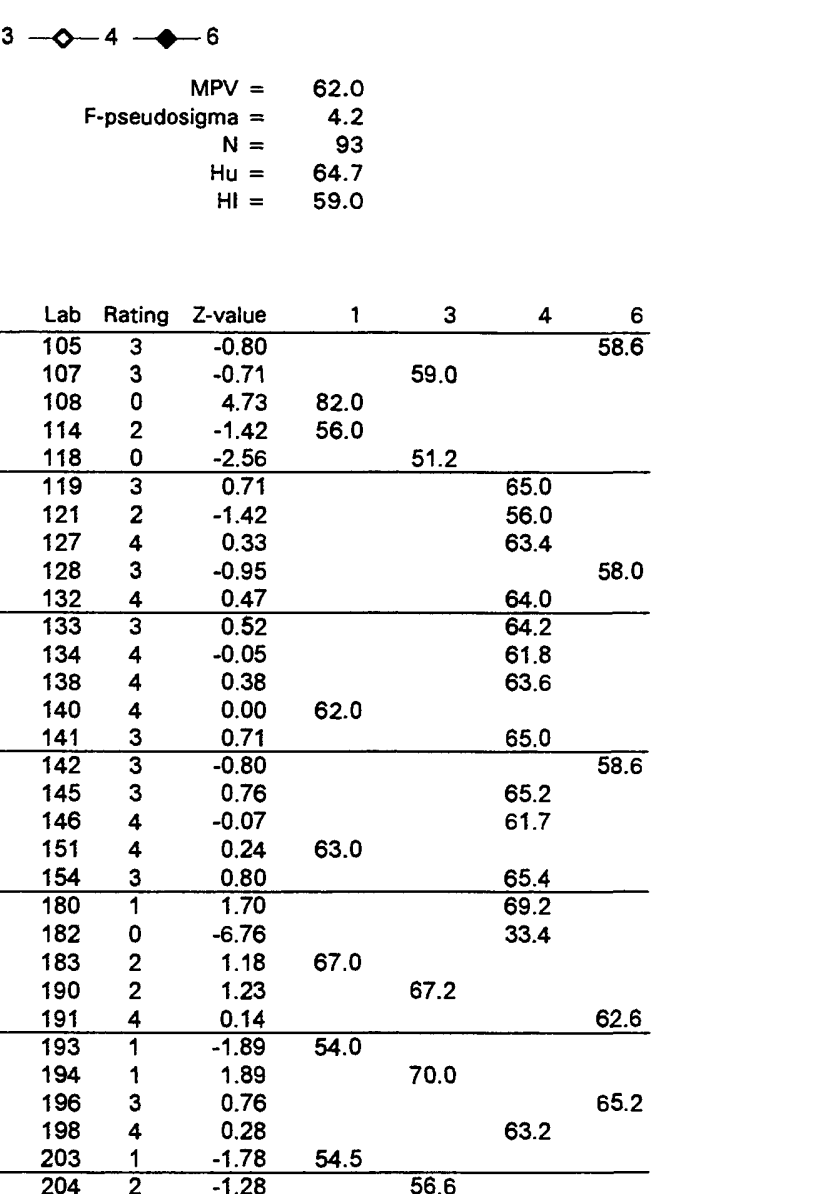

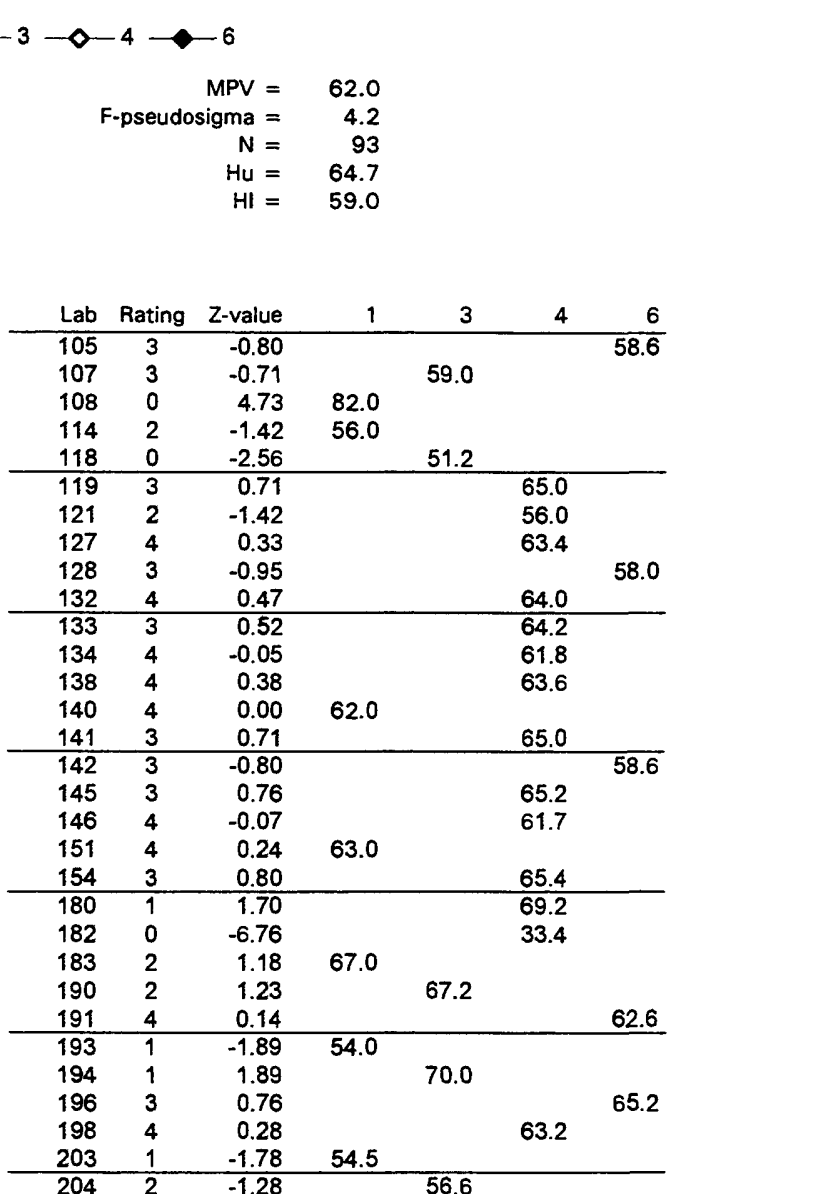
1832

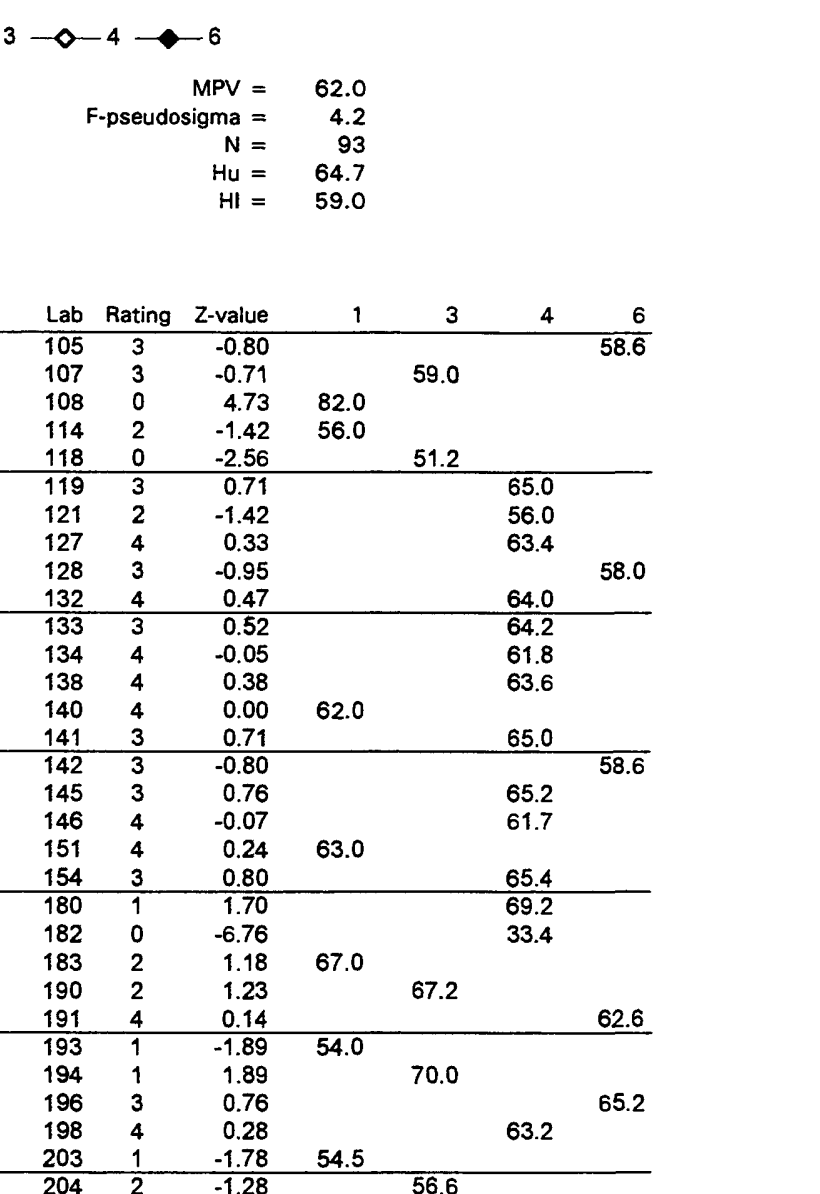

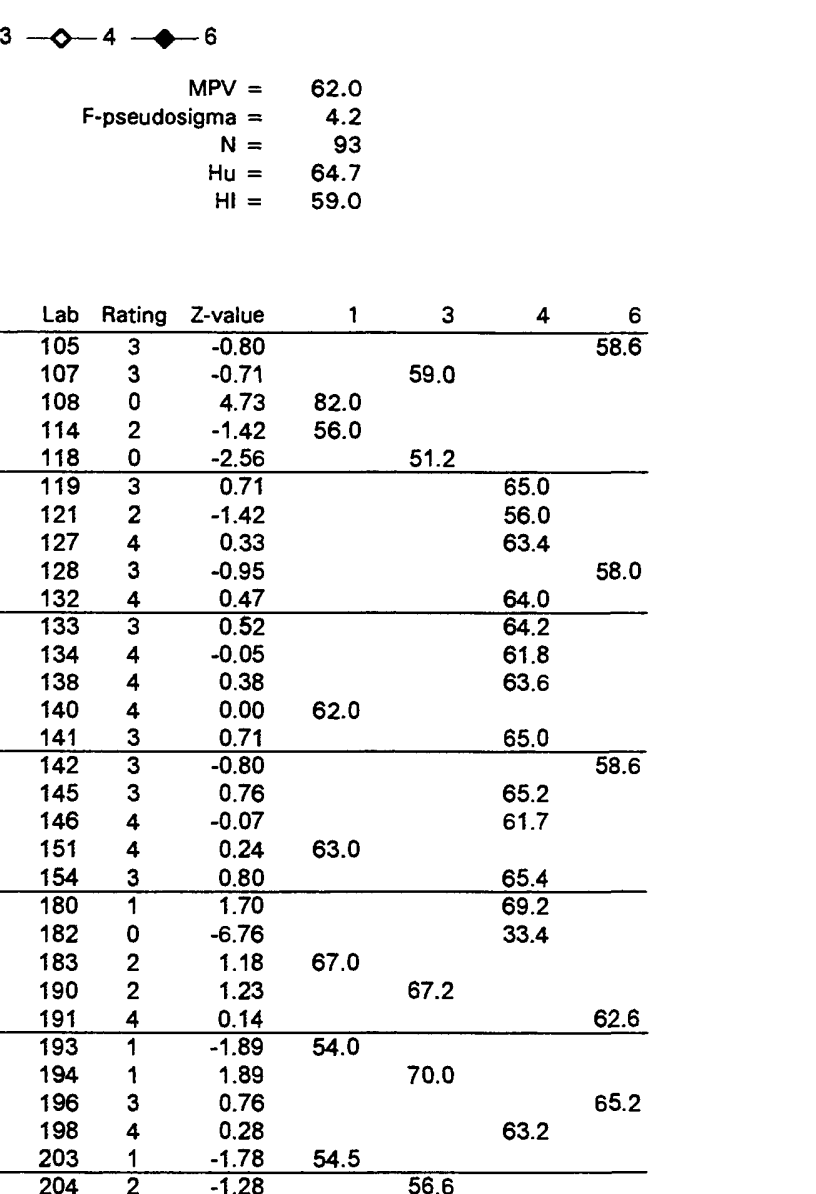

$\begin{array}{lll}198 & 4 & 0.28\end{array}$

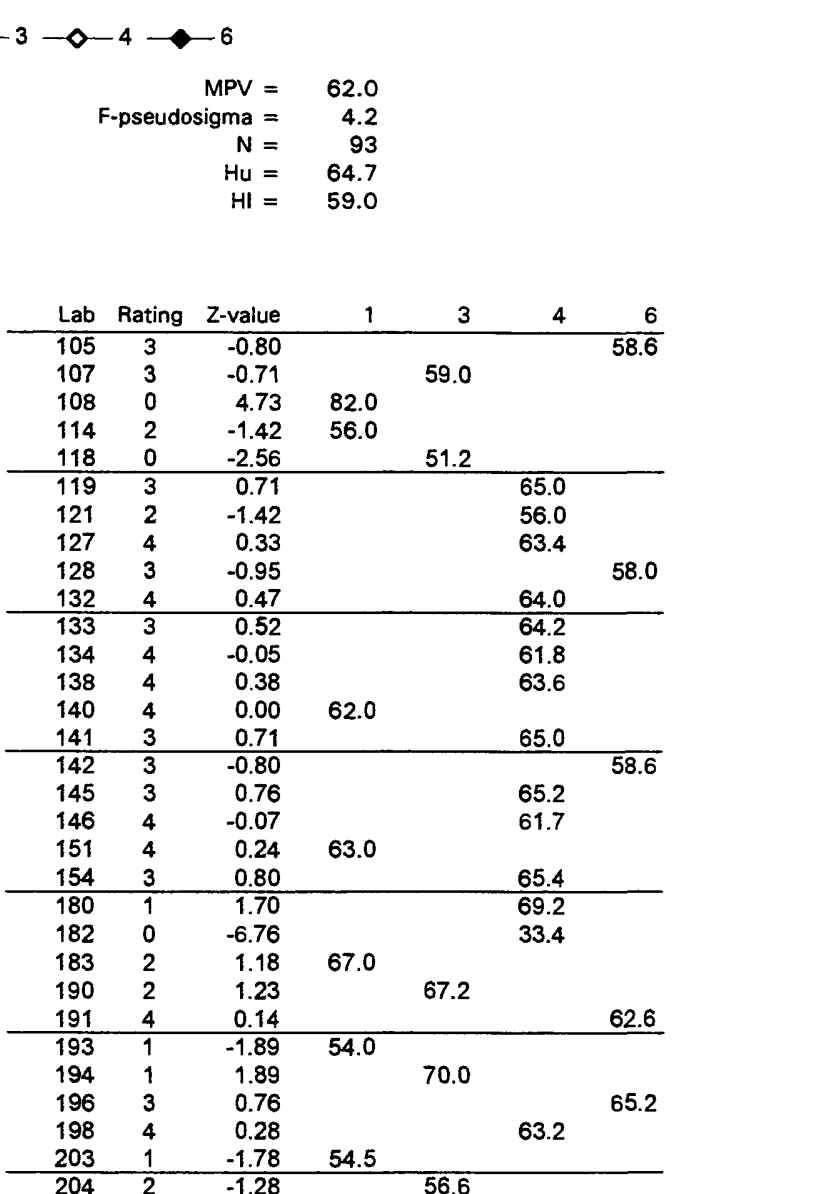

\begin{tabular}{rrrrrr}
204 & 2 & -1.28 & 56.6 & & \\
210 & 4 & -0.47 & & 60.0 & \\
212 & 1 & 1.89 & & 70.0 & \\
213 & 3 & 0.64 & 64.7 & & \\
215 & 4 & 0.47 & & 64.0 & \\
\hline 219 & 4 & -0.24 & & & 61.0 \\
221 & 4 & 0.00 & 62.0 & & \\
224 & 2 & -1.18 & & 57.0 & \\
231 & 0 & -2.18 & 52.8 & & \\
234 & 3 & 0.73 & & 65.1 & \\
\hline 235 & 3 & -0.71 & 59.0 & & \\
236 & 4 & 0.24 & & 63.0 & \\
241 & 4 & 0.43 & 63.8 & &
\end{tabular}


Table 12. Statistical summary of reported data for standard reference water sample T-135 (trace constituents)--Continued Fe (Iron) $\mu \mathrm{g} / \mathrm{l}$

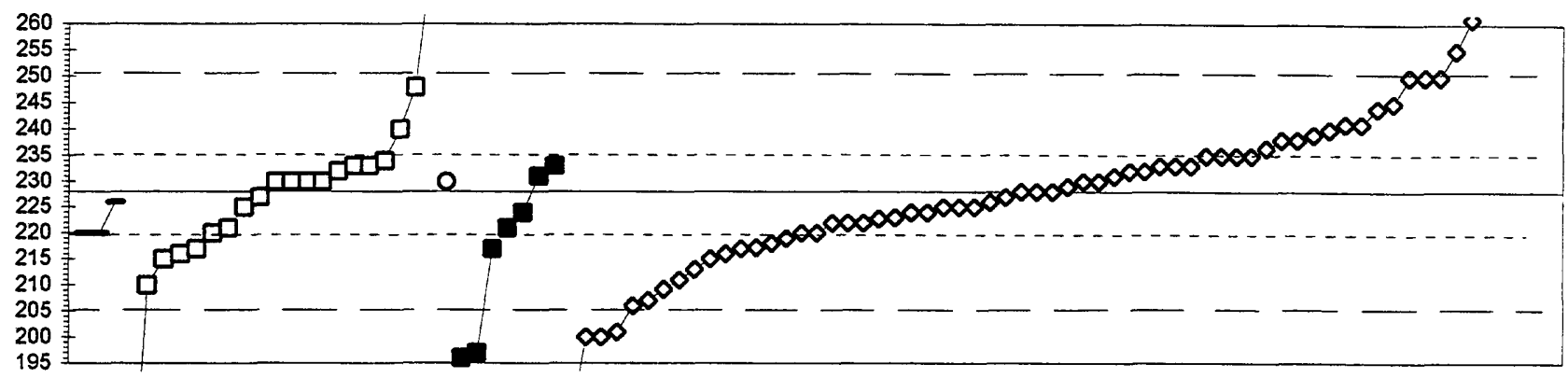

$$
-0 \rightarrow \square-1-0-2 \rightarrow-3-0-4 \rightarrow-6
$$

\begin{tabular}{|c|c|c|c|c|c|c|c|c|}
\hline \multirow[b]{7}{*}{ Lab } & \multicolumn{3}{|c|}{$\begin{array}{l}\text { 0. Other } \\
\text { 1. AA: direct air }\end{array}$} & \multicolumn{4}{|c|}{$\begin{array}{l}\text { 3. AA: graphite furnace } \\
\text { 4. ICP } \\
\text { 6. ICP/MS }\end{array}$} & \multirow{6}{*}{$\begin{array}{r}3 \\
265 \\
300 \\
270\end{array}$} \\
\hline & & $N=$ & 3 & 20 & 1 & 7 & 61 & \\
\hline & & imum = & 220 & 160 & 230 & 196 & 182 & \\
\hline & Ma & imum = & 226 & 274 & & 233 & 465 & \\
\hline & & edian $=$ & 220 & 230 & & 221 & 228 & \\
\hline & \multicolumn{2}{|c|}{ F-pseudosigma $=$} & & 11 & & 15 & 12 & \\
\hline & Rating & Z-value & 0 & 1 & 2 & 3 & 4 & 6 \\
\hline 1 & 3 & 0.63 & & & & & 235 & \\
\hline 3 & 1 & 1.98 & & & & & 250 & \\
\hline 4 & 0 & 21.31 & & & & & 465 & \\
\hline 7 & 3 & -0.72 & & & & & 220 & \\
\hline 10 & 4 & 0.18 & & 230 & & & & \\
\hline 11 & 1 & 1.98 & & & & & 250 & \\
\hline 13 & 3 & 0.90 & & & & & 238 & \\
\hline 15 & 3 & -0.54 & & & & & 222 & \\
\hline 16 & 1 & -1.53 & & & & & 211 & \\
\hline 18 & 4 & 0.00 & & & & & 228 & \\
\hline 19 & 2 & 1.17 & & & & & 241 & \\
\hline 21 & 3 & -0.72 & 220 & & & & & \\
\hline 24 & 3 & -0.90 & & & & & 218 & \\
\hline 25 & 0 & -2.43 & & & & & 201 & \\
\hline 26 & 4 & 0.00 & & & & & 228 & \\
\hline 28 & 1 & -1.69 & & & & & 209 & \\
\hline 32 & 0 & 3.78 & & & & & & 270 \\
\hline 33 & 3 & -0.72 & 220 & & & & & \\
\hline 35 & 4 & -0.18 & 226 & & & & & \\
\hline 36 & 2 & -1.08 & & 216 & & & & \\
\hline 39 & 2 & -1.35 & & & & & 213 & \\
\hline 40 & 4 & 0.27 & & & & & 231 & \\
\hline 42 & 3 & 0.63 & & & & & 235 & \\
\hline 43 & 4 & 0.18 & & & & & 230 & \\
\hline 46 & 4 & 0.09 & & & & & 229 & \\
\hline 48 & 0 & -2.52 & & & & & 200 & \\
\hline 50 & 0 & -2.88 & & & & 196 & & \\
\hline 52 & 3 & -0.72 & & & & & 220 & \\
\hline 54 & 4 & 0.18 & & 230 & & & & \\
\hline 55 & 4 & -0.45 & & & & & 223 & \\
\hline 58 & 0 & -6.12 & & 160 & & & & \\
\hline 59 & 4 & -0.18 & & & & & 226 & \\
\hline 68 & 0 & -2.52 & & & & & 200 & \\
\hline 69 & 4 & 0.36 & & 232 & & & & \\
\hline 70 & 4 & -0.27 & & & & & 225 & \\
\hline 73 & 3 & 0.76 & & & & & 237 & \\
\hline 75 & 3 & -0.81 & & & & & 219 & \\
\hline 76 & 4 & 0.45 & & 233 & & & & \\
\hline 80 & 2 & 1.08 & & 240 & & & & \\
\hline 81 & 0 & -4.14 & & & & & 182 & \\
\hline 83 & 3 & -0.54 & & & & & 222 & \\
\hline 85 & 4 & 0.36 & & & & & 232 & \\
\hline 86 & 4 & 0.36 & & & & & 232 & \\
\hline 87 & 4 & 0.18 & & & 230 & & & \\
\hline 89 & 4 & 0.27 & & & & 231 & & \\
\hline 90 & 4 & 0.45 & & 233 & & & & \\
\hline 91 & 4 & -0.09 & & & & & 227 & \\
\hline 92 & 2 & -1.17 & & 215 & & & & \\
\hline 96 & 3 & -0.72 & & 220 & & & & \\
\hline 97 & 3 & -0.99 & & & & 217 & & \\
\hline
\end{tabular}

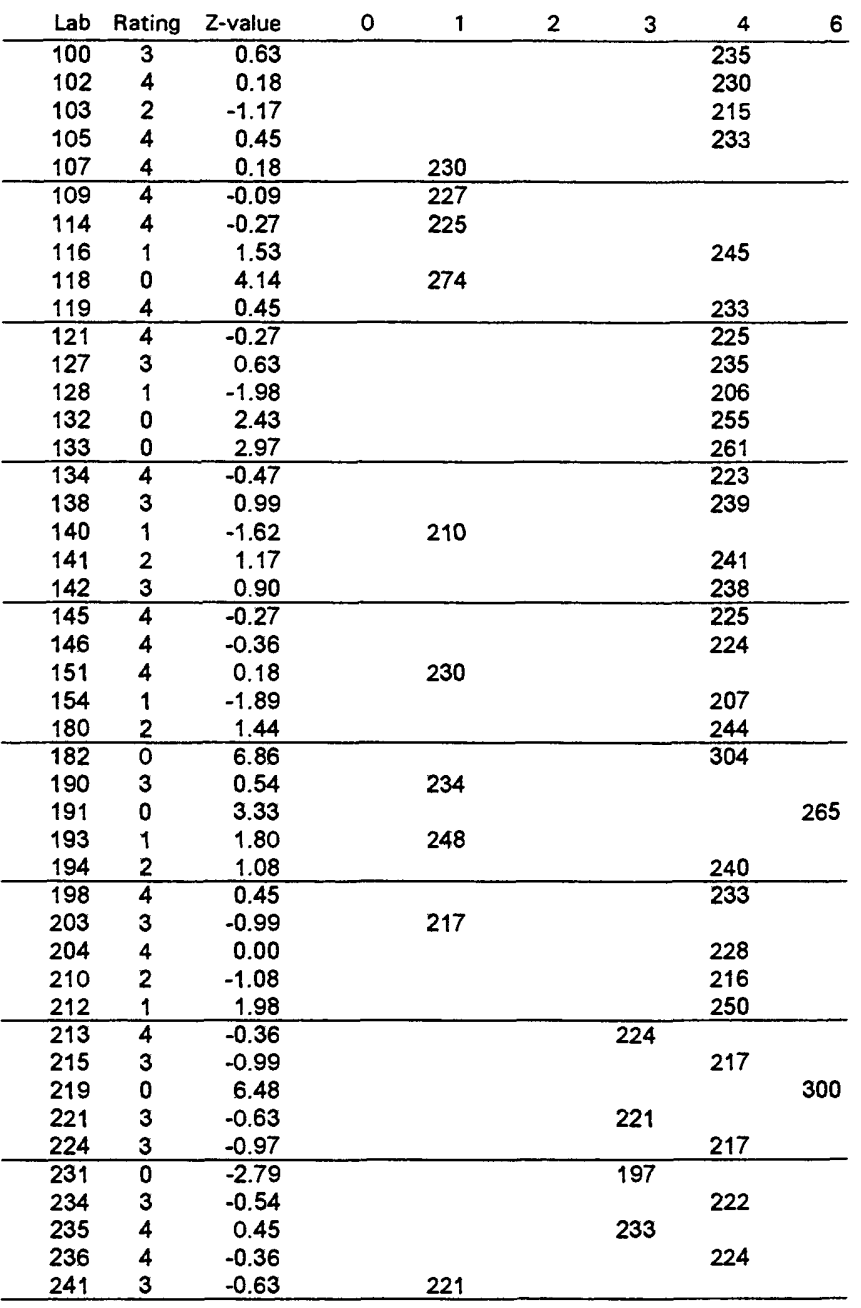


Table 12. Statistical summary of reported data for standard reference water sample T-135 (trace constituents)--Continued K (Potassium) $\mathrm{mg} / \mathrm{l}$

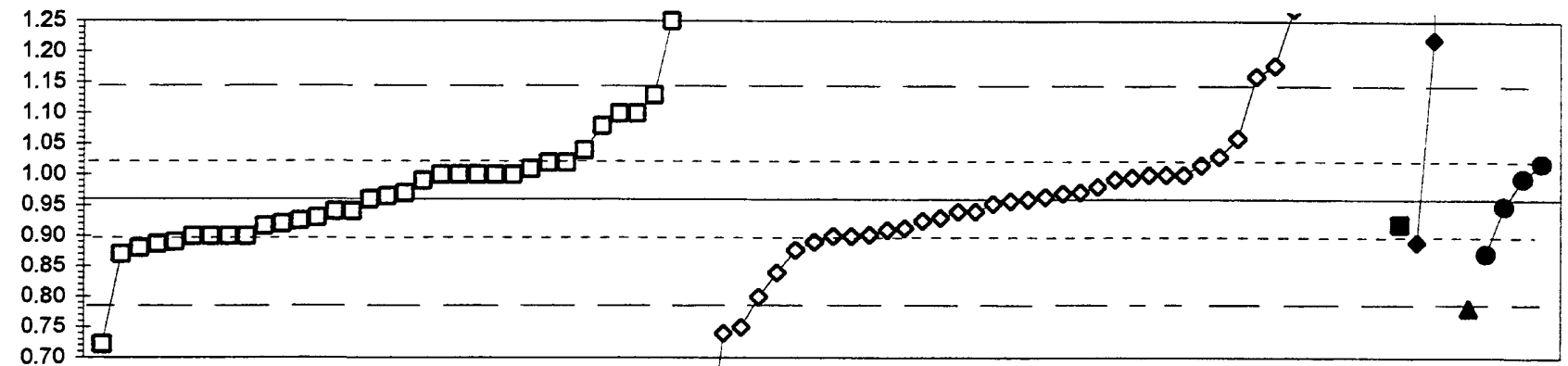

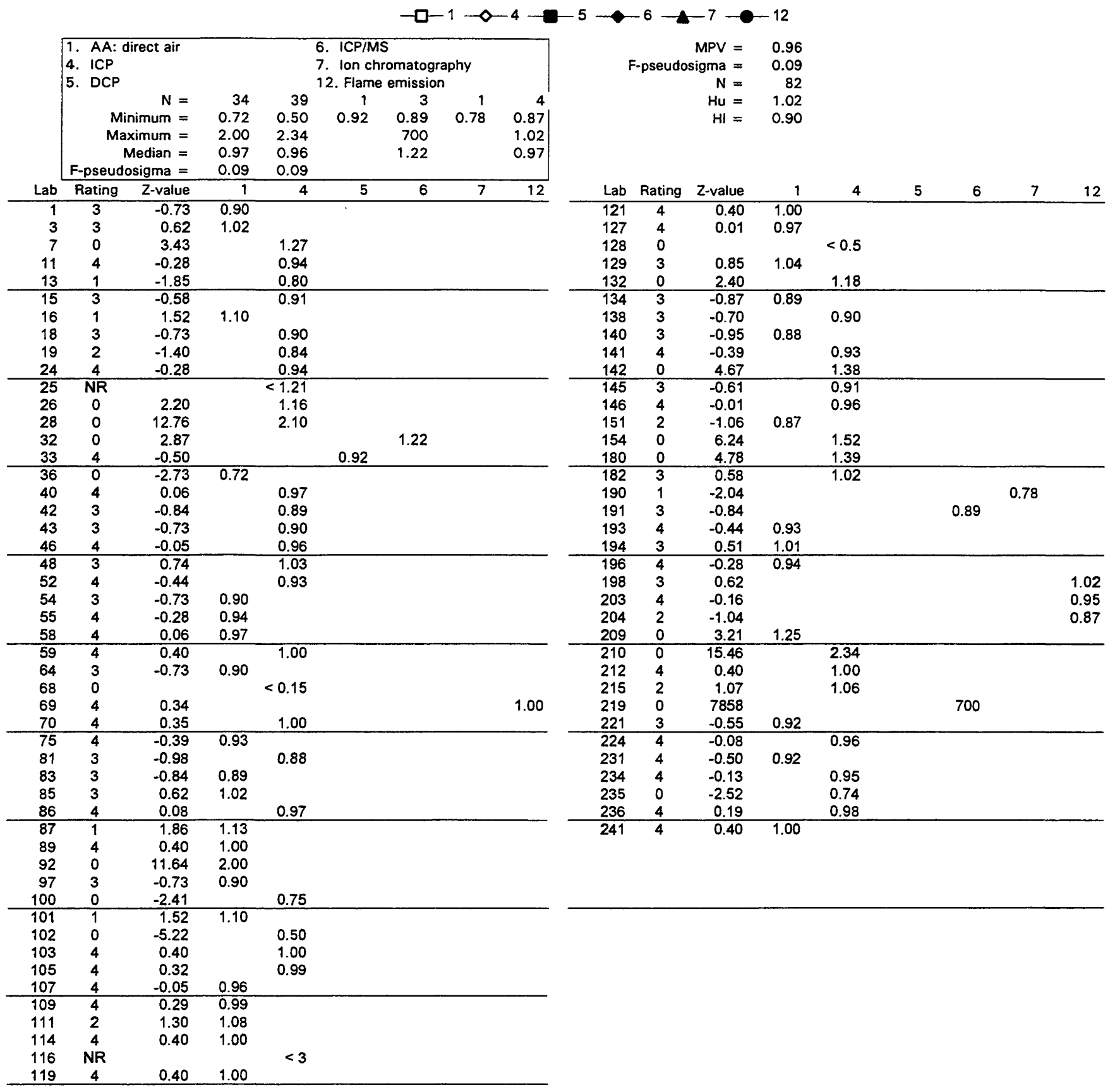


Table 12. Statistical summary of reported data for standard reference water sample T-135 (trace constituents)--Continued Li (Lithium) $\mu g / l$

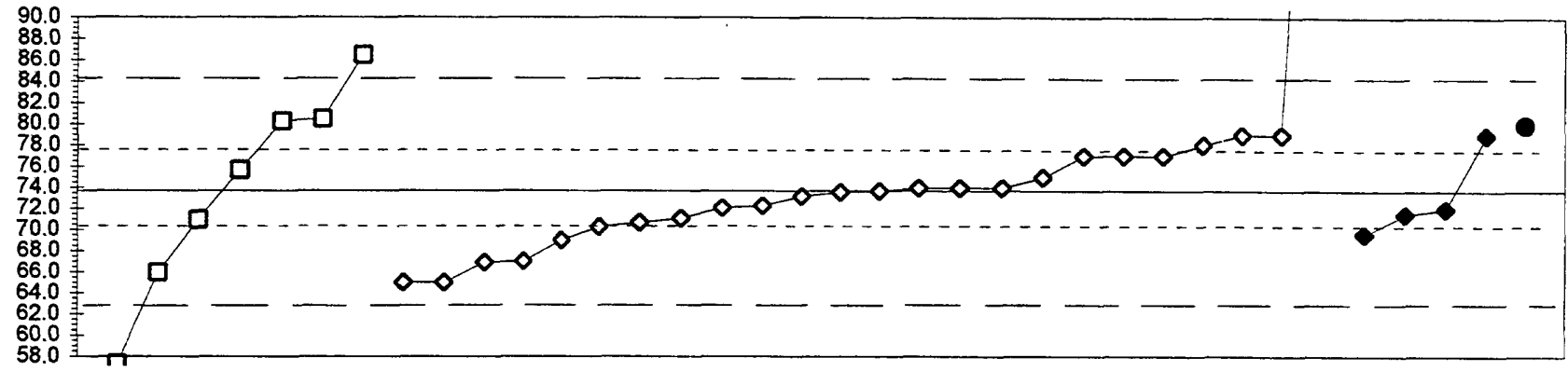

$-\square-1 \multimap 4 \multimap 6 \multimap-12$

\begin{tabular}{|c|c|c|c|c|c|c|}
\hline & \multicolumn{2}{|c|}{$\begin{array}{ll}\text { 1. } & \text { AA: direct air } \\
\text { 4. } & \text { ICP } \\
\text { 6. } & \text { ICP/MS }\end{array}$} & \multicolumn{4}{|c|}{ 12. Flame emission } \\
\hline & \multirow{2}{*}{\multicolumn{2}{|c|}{$\begin{aligned} N & = \\
\text { Minimum } & =\end{aligned}$}} & 7 & 24 & & 1 \\
\hline & & & 57.3 & 65.0 & 69.6 & 80.0 \\
\hline & \multicolumn{2}{|c|}{ Maximum $=$} & 86.5 & 142.0 & 79.0 & \\
\hline & \multirow{2}{*}{\multicolumn{2}{|c|}{$\begin{aligned} \text { Median } & = \\
\text { F-pseudosigma } & =\end{aligned}$}} & 75.7 & 73.7 & 71.8 & \\
\hline & & & 8.8 & 4.8 & & \\
\hline Lab & Rating & Z-value & 1 & 4 & 6 & 12 \\
\hline 1 & 4 & -0.01 & & 73.6 & & \\
\hline 3 & 3 & 0.64 & & 77.0 & & \\
\hline 4 & 0 & 13.08 & & 142.0 & & \\
\hline 7 & 4 & -0.26 & & 72.3 & & \\
\hline 15 & 3 & 0.85 & & 78.1 & & \\
\hline 16 & 3 & 0.64 & & 77.0 & & \\
\hline 24 & 4 & 0.07 & & 74.0 & & \\
\hline 25 & 2 & -1.27 & & 67.0 & & \\
\hline 26 & 4 & 0.07 & & 74.0 & & \\
\hline 28 & 0 & 2.46 & 86.5 & & & \\
\hline 30 & 3 & -0.77 & & & 69.6 & \\
\hline 32 & 2 & 1.02 & & & 79.0 & \\
\hline 39 & 3 & -0.56 & & 70.7 & & \\
\hline 40 & 4 & 0.26 & & 75.0 & & \\
\hline 42 & 4 & -0.09 & & 73.2 & & \\
\hline 55 & 2 & -1.46 & 66.0 & & & \\
\hline 64 & 2 & 1.22 & & & & 80.0 \\
\hline 68 & 1 & -1.66 & & 65.0 & & \\
\hline 69 & 2 & 1.31 & 80.5 & & & \\
\hline 75 & 3 & -0.64 & & 70.3 & & \\
\hline 85 & 4 & 0.39 & 75.7 & & & \\
\hline 100 & 2 & 1.27 & 80.3 & & & \\
\hline 103 & 3 & -0.89 & & 69.0 & & \\
\hline 105 & 2 & 1.02 & & 79.0 & & \\
\hline 109 & 0 & -3.12 & 57.3 & & & \\
\hline 116 & NR & & & $<80$ & & \\
\hline 127 & 2 & -1.29 & & 66.9 & & \\
\hline 134 & 2 & 1.02 & & 79.0 & & \\
\hline 142 & 4 & 0.01 & & 73.7 & & \\
\hline 145 & 4 & -0.49 & & 71.1 & & \\
\hline 151 & 3 & -0.51 & 71.0 & & & \\
\hline 182 & 1 & -1.66 & & 65.0 & & \\
\hline 196 & 4 & -0.41 & & & 71.5 & \\
\hline 212 & 3 & 0.64 & & 77.0 & & \\
\hline 219 & 4 & -0.32 & & & 72.0 & \\
\hline 234 & 4 & -0.30 & & 72.1 & & \\
\hline 236 & 4 & 0.07 & & 74.0 & & \\
\hline
\end{tabular}


Table 12. Statistical summary of reported data for standard reference water sample T-135 (trace constituents)--Continued Mg (Magnesium) $\mathrm{mg} / \mathrm{l}$

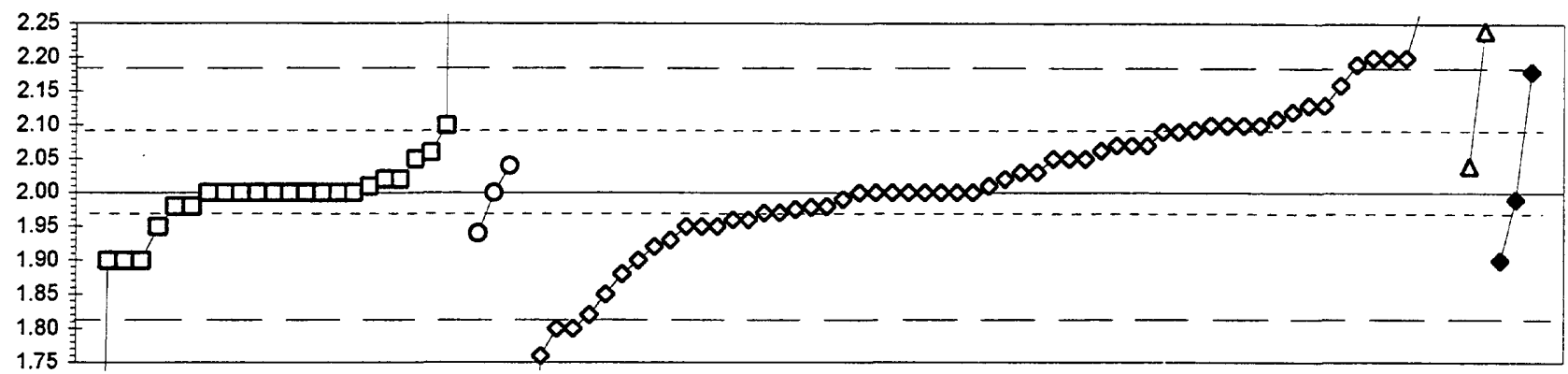

\begin{tabular}{|c|c|c|c|c|c|c|c|c|c|c|c|c|c|c|c|c|c|}
\hline \multirow[b]{2}{*}{ Lab } & 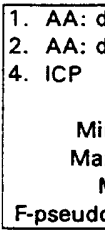 & $\begin{array}{r}\text { rect air } \\
N= \\
\text { imum }= \\
\text { imum }= \\
\text { edian }= \\
\text { sigma }=\end{array}$ & $\begin{array}{r}\text { s oxide } \\
\\
24 \\
0.31 \\
25.00 \\
2.00 \\
0.03 \\
\end{array}$ & $\begin{array}{r}3 \\
1.94 \\
2.04 \\
2.00\end{array}$ & $\begin{array}{c}D C P \\
\text { ICP/N } \\
\text { Ion } \mathrm{cl} \\
59 \\
1.05 \\
4.23 \\
2.01 \\
0.10 \\
\end{array}$ & $\begin{array}{c} \\
\\
\text { omatog } \\
2 \\
2.04 \\
2.24 \\
2.14\end{array}$ & $\begin{array}{r}\text { phy } \\
3 \\
1.90 \\
2.18 \\
1.99\end{array}$ & $\begin{array}{r}1 \\
2.29\end{array}$ & \multicolumn{3}{|c|}{$\begin{aligned} M P V & = \\
\text { F-pseudosigma } & = \\
N & = \\
H u= & \\
H I & =\end{aligned}$} & $\begin{array}{r}2.00 \\
0.09 \\
92 \\
2.09 \\
1.97\end{array}$ & \multirow[b]{2}{*}{2} & \multirow[b]{2}{*}{4} & \multirow[b]{2}{*}{5} & \multirow[b]{2}{*}{6} & \multirow[b]{2}{*}{7} \\
\hline & Rating & Z-value & 1 & 2 & 4 & 5 & 6 & 7 & Lab & Rating & Z-value & 1 & & & & & \\
\hline 1 & 4 & 0.22 & 2.02 & & & & & & 109 & 4 & 0.00 & 2.00 & & & & & \\
\hline 3 & 4 & 0.00 & & & 2.00 & & & & 111 & 4 & 0.45 & & 2.04 & & & & \\
\hline 4 & 0 & 25.07 & & & 4.23 & & & & 114 & 4 & 0.00 & & 2.00 & & & & \\
\hline 7 & 4 & 0.00 & & & 2.00 & & & & 116 & 4 & -0.45 & & & 1.96 & & & \\
\hline 11 & 1 & -2.02 & & & 1.82 & & & & 119 & 0 & 3.37 & & & 2.30 & & & \\
\hline 13 & 2 & 1.12 & & & 2.10 & & & & 121 & 4 & 0.34 & & & 2.03 & & & \\
\hline 15 & 2 & 1.35 & & & 2.12 & & & & 127 & 3 & 0.79 & & & 2.07 & & & \\
\hline 16 & 4 & 0.00 & & & 2.00 & & & & 128 & 1 & -1.69 & & & 1.85 & & & \\
\hline 18 & 4 & 0.00 & & & 2.00 & & & & 129 & 0 & -19.05 & 0.31 & & & & & \\
\hline 19 & 0 & 3.15 & & & 2.28 & & & & 132 & 3 & 0.70 & & & 2.06 & & & \\
\hline 24 & 4 & 0.00 & & & 2.00 & & & & 133 & 3 & -0.79 & & & 1.93 & & & \\
\hline 25 & 3 & -0.90 & & & 1.92 & & & & 134 & 3 & 0.56 & & & 2.05 & & & \\
\hline 26 & 3 & 0.79 & & & 2.07 & & & & 138 & 2 & 1.01 & & & 2.09 & & & \\
\hline 27 & 0 & 2.70 & & & & 2.24 & & & 140 & 4 & 0.00 & 2.00 & & & & & \\
\hline 28 & 2 & 1.12 & & & 2.10 & & & & 141 & 2 & 1.46 & & & 2.13 & & & \\
\hline 30 & 3 & -0.67 & & 1.94 & & & & & 142 & 4 & 0.34 & & & 2.03 & & & \\
\hline 32 & 1 & 2.02 & & & & & 2.18 & & 145 & 4 & 0.22 & & & 2.02 & & & \\
\hline 33 & 4 & 0.45 & & & & 2.04 & & & 146 & 4 & -0.45 & & & 1.96 & & & \\
\hline 36 & 3 & 0.67 & 2.06 & & & & & & 151 & 4 & 0.00 & 2.00 & & & & & \\
\hline 39 & 4 & -0.34 & & & 1.97 & & & & 154 & 0 & -2.25 & & & 1.80 & & & \\
\hline 40 & 4 & 0.00 & & & 2.00 & & & & 180 & 0 & 2.25 & & & 2.20 & & & \\
\hline 42 & 2 & 1.12 & & & 2.10 & & & & 182 & 4 & -0.28 & & & 1.98 & & & \\
\hline 43 & 4 & 0.00 & & & 2.00 & & & & 190 & 0 & 3.26 & & & & & & 2.29 \\
\hline 46 & 2 & 1.01 & & & 2.09 & & & & 191 & 4 & -0.11 & & & & & 1.99 & \\
\hline 48 & 3 & 0.56 & & & 2.05 & & & & 193 & 4 & -0.22 & 1.98 & & & & & \\
\hline 52 & 4 & 0.00 & & & 2.00 & & & & 194 & 2 & -1.35 & & & 1.88 & & & \\
\hline 54 & 4 & 0.00 & 2.00 & & & & & & 196 & 2 & -1.12 & 1.90 & & & & & \\
\hline 55 & 0 & 2.14 & & & 2.19 & & & & 198 & 1 & 1.80 & & & 2.16 & & & \\
\hline 58 & 0 & 258.56 & 25.00 & & & & & & 203 & 4 & 0.22 & 2.02 & & & & & \\
\hline 59 & 0 & 2.25 & & & 2.20 & & & & 204 & 4 & 0.11 & & & 2.01 & & & \\
\hline 64 & 3 & -0.56 & & & 1.95 & & & & 209 & 2 & 1.24 & & & 2.11 & & & \\
\hline 68 & 0 & -10.68 & & & 1.05 & & & & 210 & 0 & -2.70 & & & 1.76 & & & \\
\hline 69 & 2 & 1.12 & 2.10 & & & & & & 212 & 0 & 2.25 & & & 2.20 & & & \\
\hline 70 & 2 & 1.46 & & & 2.13 & & & & 215 & 4 & -0.22 & & & 1.98 & & & \\
\hline 75 & 4 & 0.00 & 2.00 & & & & & & 219 & 2 & -1.12 & & & & & 1.90 & \\
\hline 81 & 3 & -0.56 & & & 1.95 & & & & 221 & 3 & -0.56 & 1.95 & & & & & \\
\hline 83 & 4 & -0.11 & & & 1.99 & & & & 224 & 2 & 1.05 & & & 2.09 & & & \\
\hline 84 & 4 & 0.00 & 2.00 & & & & & & 231 & 3 & 0.56 & 2.05 & & & & & \\
\hline 85 & 4 & -0.22 & 1.98 & & & & & & 234 & 3 & -0.56 & & & 1.95 & & & \\
\hline 86 & 3 & 0.79 & & & 2.07 & & & & 235 & 4 & -0.34 & & & 1.97 & & & \\
\hline 87 & 2 & -1.12 & 1.90 & & & & & & 236 & 4 & -0.24 & & & 1.98 & & & \\
\hline 89 & 4 & 0.00 & 2.00 & & & & & & 241 & 2 & -1.12 & 1.90 & & & & & \\
\hline 92 & 4 & 0.00 & 2.00 & & & & & & & & & & & & & & \\
\hline 97 & 4 & 0.00 & 2.00 & & & & & & & & & & & & & & \\
\hline 100 & 2 & 1.12 & & & 2.10 & & & & & & & & & & & & \\
\hline 101 & 4 & 0.00 & 2.00 & & & & & & & & & & & & & & \\
\hline 102 & 0 & -2.25 & & & 1.80 & & & & & & & & & & & & \\
\hline 103 & 2 & -1.12 & & & 1.90 & & & & & & & & & & & & \\
\hline 105 & 3 & 0.56 & & & 2.05 & & & & & & & & & & & & \\
\hline 107 & 4 & 0.11 & 2.01 & & & & & & & & & & & & & & \\
\hline
\end{tabular}


Table 12. Statistical summary of reported data for standard reference water sample T-135 (trace constituents)--Continued Mn (Manganese) $\mu \mathrm{g} / \mathrm{I}$

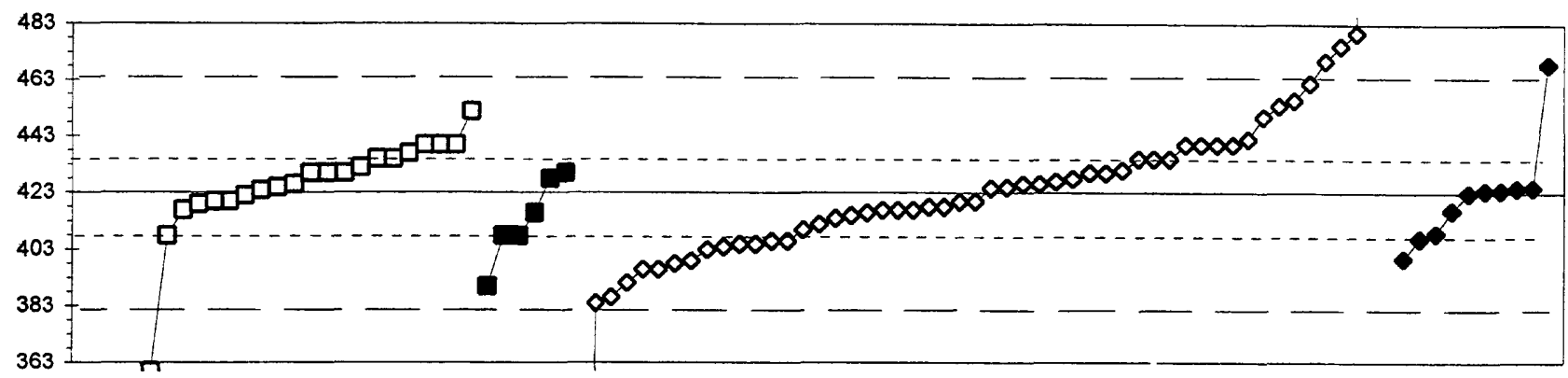

\begin{tabular}{|c|c|c|c|c|c|c|c|c|c|c|c|c|c|c|c|}
\hline \multirow[b]{2}{*}{ Lab } & $\begin{array}{l}\text { 1. AA: } \\
\text { 3. AA: } \\
\text { 4. ICP } \\
\\
\\
\\
\text { F-pseud } \\
\end{array}$ & 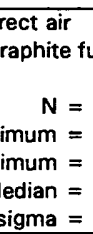 & $\begin{array}{r}\text { ace } \\
25 \\
42 \\
452 \\
425 \\
13\end{array}$ & $\begin{array}{r}6 \\
390 \\
430 \\
412\end{array}$ & $\begin{array}{r}51 \\
36 \\
825 \\
420 \\
23\end{array}$ & $\begin{array}{r}1 \\
220\end{array}$ & $\begin{array}{r}10 \\
400 \\
469 \\
424 \\
12\end{array}$ & \multirow[b]{2}{*}{ Lab } & \multicolumn{2}{|c|}{$\begin{aligned} \text { MPV } & = \\
\text { F-pseudosigma } & = \\
N & = \\
H U & = \\
H I & =\end{aligned}$} & $\begin{array}{r}423 \\
20 \\
93 \\
435 \\
408\end{array}$ & \multirow[b]{2}{*}{3} & \multirow[b]{2}{*}{4} & \multirow[b]{2}{*}{5} & \multirow[b]{2}{*}{6} \\
\hline & Rating & Z-value & 1 & 3 & 4 & 5 & 6 & & Rating & Z-value & 1 & & & & \\
\hline 1 & 4 & 0.45 & 432 & & & & & 102 & 3 & -0.90 & & & 405 & & \\
\hline 3 & 3 & -0.90 & & & 405 & & & 103 & 4 & 0.15 & & & 426 & & \\
\hline 4 & 0 & 20.09 & & & 825 & & & 105 & 4 & 0.05 & & & & & 424 \\
\hline 7 & 4 & -0.25 & & & 418 & & & 107 & 4 & 0.35 & 430 & & & & \\
\hline 10 & 4 & 0.10 & 425 & & & & & 109 & 4 & -0.30 & 417 & & & & \\
\hline 11 & 0 & 2.35 & & & 470 & & & 114 & 4 & -0.15 & 420 & & & & \\
\hline 13 & 3 & 0.85 & & & 440 & & & 116 & 4 & 0.10 & & & 425 & & \\
\hline 15 & 3 & 0.85 & & & 440 & & & 118 & 0 & -4.30 & 337 & & & & \\
\hline 16 & 2 & -1.25 & & & 398 & & & 119 & 3 & 0.95 & & & 442 & & \\
\hline 18 & 4 & 0.20 & & & 427 & & & 121 & 3 & -0.65 & & & 410 & & \\
\hline 19 & 1 & 1.55 & & & 454 & & & 127 & 4 & -0.30 & & & 417 & & \\
\hline 23 & 1 & -1.65 & & 390 & & & & 128 & 3 & -0.80 & & & & & 407 \\
\hline 24 & 3 & 0.60 & & & 435 & & & 129 & 4 & -0.15 & 420 & & & & \\
\hline 25 & 1 & -1.95 & & & 384 & & & 132 & 3 & -1.00 & & & 403 & & \\
\hline 26 & 3 & 0.85 & & & 440 & & & 134 & 4 & 0.35 & & & 430 & & \\
\hline 28 & 1 & -1.59 & & & 391 & & & 138 & 4 & -0.35 & & & 416 & & \\
\hline 30 & 4 & 0.00 & & & & & 423 & 140 & 4 & 0.35 & 430 & & & & \\
\hline 32 & 3 & -0.70 & & & & & 409 & 141 & 4 & 0.25 & & & 428 & & \\
\hline 33 & 0 & -10.14 & & & & 220 & & 142 & 4 & 0.09 & & & & & 425 \\
\hline 36 & 0 & -3.65 & 350 & & & & & 145 & 4 & 0.08 & & & 425 & & \\
\hline 39 & 1 & -1.85 & & & 386 & & & 146 & 4 & -0.25 & & & 418 & & \\
\hline 40 & 3 & 0.60 & & & 435 & & & 151 & 3 & 0.60 & 435 & & & & \\
\hline 42 & 4 & 0.05 & & & & & 424 & 154 & 2 & -1.35 & & & 396 & & \\
\hline 43 & 4 & 0.35 & & & 430 & & & 180 & 1 & 1.95 & & & 462 & & \\
\hline 46 & 4 & -0.16 & & & 420 & & & 182 & 0 & 2.62 & & & 475 & & \\
\hline 48 & 3 & 0.85 & & & 440 & & & 190 & 4 & -0.35 & & 416 & & & \\
\hline 50 & 4 & 0.25 & & 428 & & & & 191 & 4 & -0.30 & & & & & 417 \\
\hline 52 & 4 & -0.15 & & & 420 & & & 194 & 2 & 1.35 & & & 450 & & \\
\hline 54 & 4 & 0.35 & 430 & & & & & 196 & $\overrightarrow{0}$ & 2.30 & & & & & 469 \\
\hline 55 & 4 & 0.40 & & & 431 & & & 198 & 1 & 1.65 & & & 456 & & \\
\hline 58 & 3 & 0.85 & 440 & & & & & 203 & 0 & -3.15 & 360 & & & & \\
\hline 59 & 4 & 0.10 & & & & & 425 & 204 & 4 & -0.30 & & & 417 & & \\
\hline 68 & 0 & -19.36 & & & 36 & & & 210 & 3 & -0.85 & & & 406 & & \\
\hline 69 & 4 & 0.15 & 426 & & & & & 212 & 0 & 2.85 & & & 480 & & \\
\hline 70 & 4 & -0.40 & & & 415 & & & 215 & 3 & -0.85 & & & 406 & & \\
\hline 75 & 4 & -0.45 & & & 414 & & & 219 & 2 & -1.15 & & & & & 400 \\
\hline 76 & 3 & 0.60 & 435 & & & & & 221 & 3 & 0.85 & 440 & & & & \\
\hline 80 & 2 & 1.45 & 452 & & & & & 224 & 2 & -1.35 & & & 396 & & \\
\hline 81 & 2 & -1.20 & & & 399 & & & 231 & 3 & -0.75 & & 408 & & & \\
\hline 83 & 3 & -0.55 & & & 412 & & & 234 & 3 & -0.95 & & & 404 & & \\
\hline 85 & 4 & -0.05 & 422 & & & & & 235 & 4 & 0.35 & & 430 & & & \\
\hline 86 & 4 & 0.15 & & & 426 & & & 236 & 4 & -0.30 & & & 417 & & \\
\hline 87 & 3 & -0.75 & 408 & & & & & 241 & 4 & -0.20 & 419 & & & & \\
\hline 89 & 4 & 0.05 & 424 & & & & & & & & & & & & \\
\hline 90 & 0 & -3.55 & 352 & & & & & & & & & & & & \\
\hline 91 & 3 & 0.60 & & & 435 & & & & & & & & & & \\
\hline 92 & 0 & -19.04 & 42 & & & & & & & & & & & & \\
\hline 96 & 3 & 0.85 & 440 & & & & & & & & & & & & \\
\hline 97 & 3 & -0.75 & & 408 & & & & & & & & & & & \\
\hline 100 & 3 & 0.70 & 437 & & & & & & & & & & & & \\
\hline
\end{tabular}


Table 12. Statistical summary of reported data for standard reference water sample T-135 (trace constituents)--Continued Mo (Molybdenum) $\mu \mathrm{g} / \mathrm{l}$
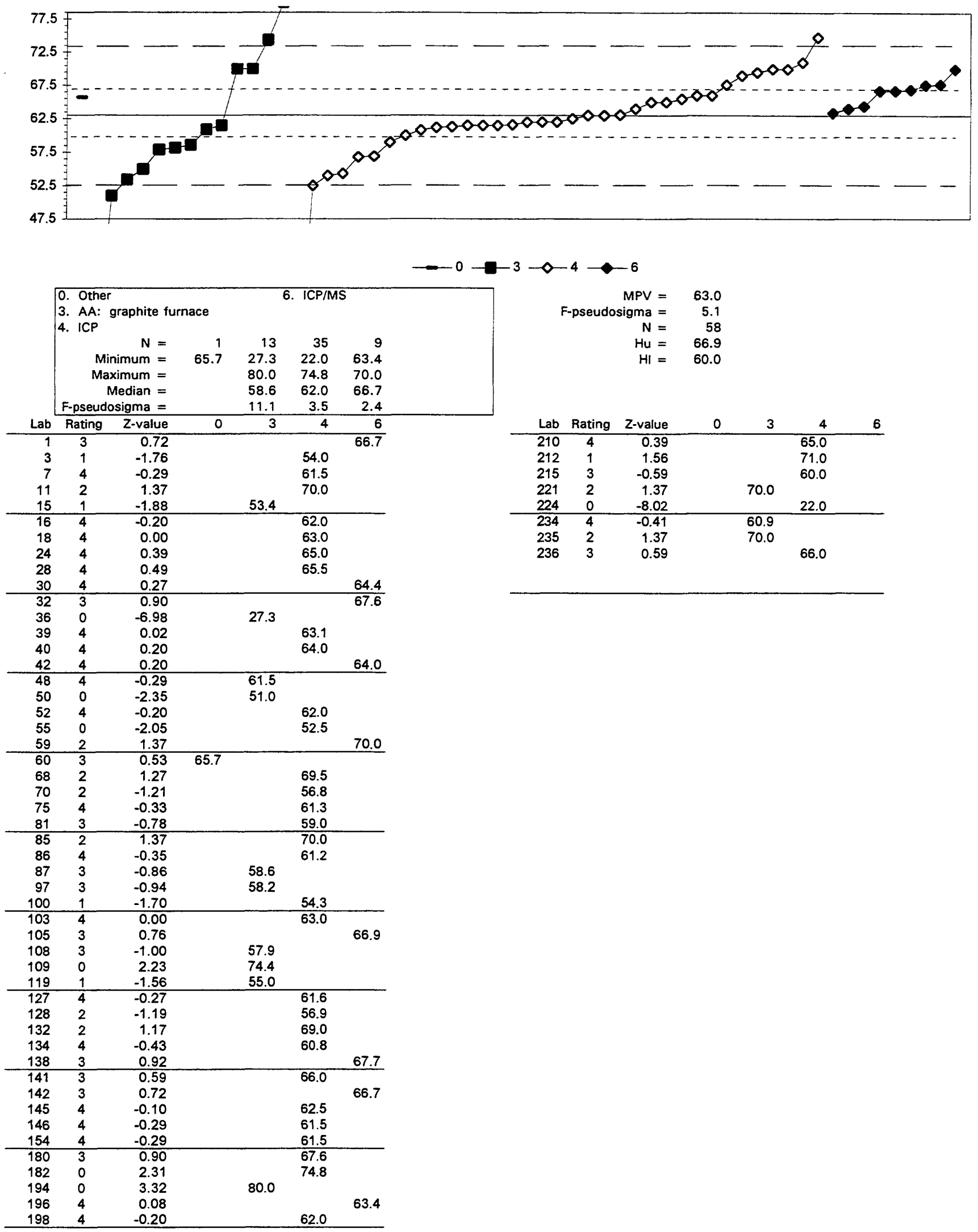
Table 12. Statistical summary of reported data for standard reference water sample T-135 (trace constituents)--Continued $\mathrm{Na}$ (Sodium) $\mathrm{mg} / \mathrm{l}$

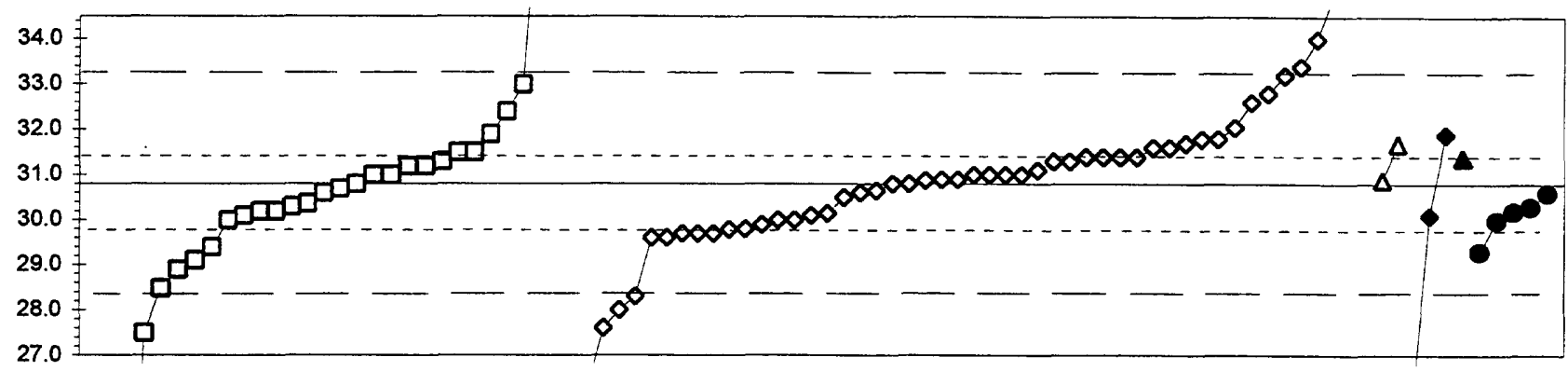

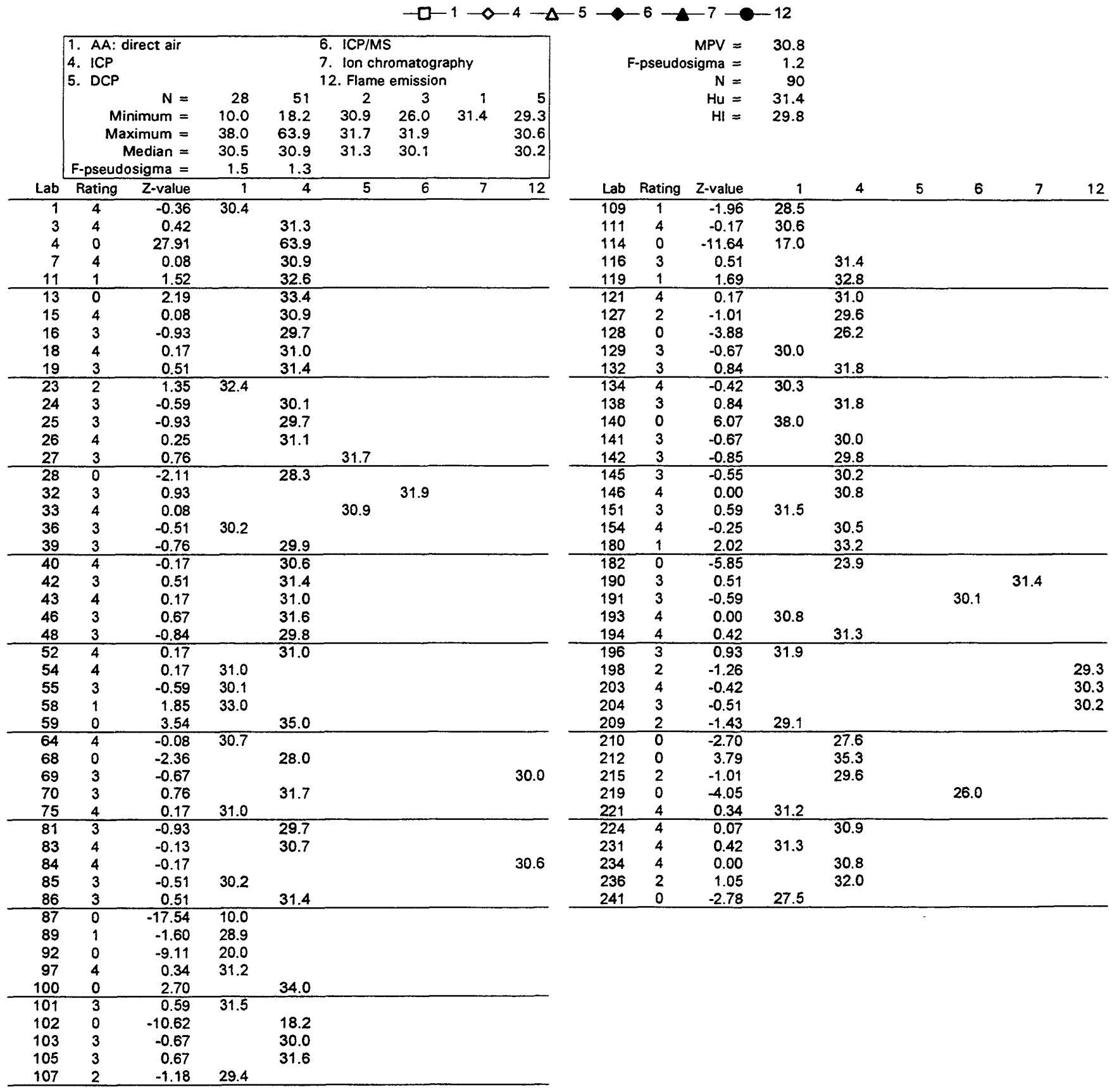


Table 12. Statistical summary of reported data for standard reference water sample T-135 (trace constituents)--Continued $\mathrm{Ni}$ (Nickel) $\mu \mathrm{g} / \mathrm{l}$

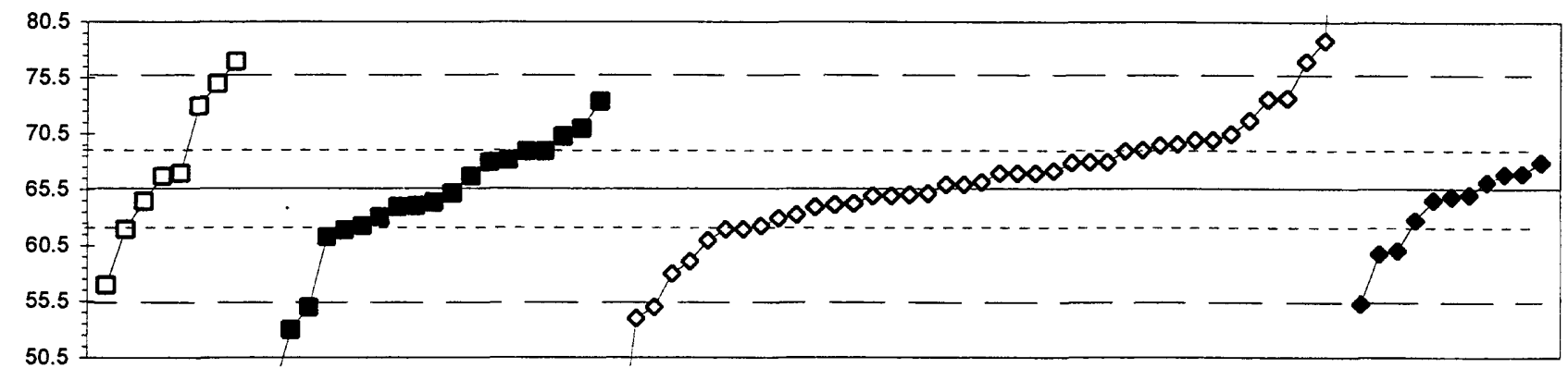

$-\square-1-3-0-4-6$

\begin{tabular}{|c|c|c|c|c|c|c|}
\hline \multirow[b]{7}{*}{ Lab } & \multicolumn{3}{|c|}{$\begin{array}{l}\text { 1. AA: direct air } \\
\text { 3. AA: graphite furnace }\end{array}$} & \multicolumn{3}{|c|}{ 6. ICP/MS } \\
\hline & & $N=$ & 8 & 20 & 41 & 11 \\
\hline & & imum = & 57.0 & 34.2 & 40.0 & 55.3 \\
\hline & & imum = & 77.0 & 73.4 & 119.0 & 68.0 \\
\hline & & ledian = & 66.9 & 64.3 & 66.2 & 64.9 \\
\hline & F-pseudo & sigma $=$ & 8.0 & 5.2 & 4.6 & 3.8 \\
\hline & Rating & Z-value & 1 & 3 & 4 & 6 \\
\hline 1 & 4 & -0.10 & & & & 65.1 \\
\hline 3 & 4 & 0.28 & & & 67.0 & \\
\hline 4 & 0 & 10.75 & & & 119.0 & \\
\hline 7 & 3 & -0.66 & & & 62.3 & \\
\hline 11 & 3 & 0.89 & & & 70.0 & \\
\hline 13 & 1 & -1.53 & & & 58.0 & \\
\hline 15 & 4 & 0.46 & & & 67.9 & \\
\hline 16 & 4 & -0.32 & & & 64.0 & \\
\hline 18 & 4 & 0.08 & & & 66.0 & \\
\hline 24 & 4 & 0.12 & & & 66.2 & \\
\hline 25 & 0 & & & & $<49$ & \\
\hline 26 & 4 & 0.22 & & 66.7 & & \\
\hline 28 & 3 & 0.68 & & & 69.0 & \\
\hline 30 & 4 & 0.26 & & & & 66.9 \\
\hline 32 & 4 & -0.20 & & & & 64.6 \\
\hline 36 & 0 & -3.72 & & 47.1 & & \\
\hline 39 & 2 & 1.23 & & & 71.7 & \\
\hline 40 & 4 & 0.28 & & & 67.0 & \\
\hline 42 & 3 & -0.56 & & & & 62.8 \\
\hline 46 & 1 & 1.61 & & & 73.6 & \\
\hline 48 & 4 & -0.08 & & 65.2 & & \\
\hline 50 & 2 & 1.09 & & 71.0 & & \\
\hline 52 & 4 & -0.12 & & & 65.0 & \\
\hline 55 & 3 & -0.66 & & 62.3 & & \\
\hline 58 & 3 & 0.68 & & 69.0 & & \\
\hline 59 & $\overline{4}$ & 0.28 & & & & 67.0 \\
\hline 60 & 0 & 2.30 & 77.0 & & & \\
\hline 68 & 0 & -2.13 & & & 55.0 & \\
\hline 69 & 4 & -0.22 & 64.5 & & & \\
\hline 70 & 3 & 0.70 & & & 69.1 & \\
\hline 73 & 4 & 0.32 & & & 67.2 & \\
\hline 75 & 4 & -0.10 & & & 65.1 & \\
\hline 76 & 4 & -0.50 & & 63.1 & & \\
\hline 81 & 0 & -5.15 & & & 40.0 & \\
\hline 83 & 3 & 0.79 & & & 69.5 & \\
\hline 85 & $\frac{4}{4}$ & 0.08 & & & 66.0 & \\
\hline 86 & 4 & -0.08 & & & 65.2 & \\
\hline 87 & 2 & 1.49 & 73.0 & & & \\
\hline 89 & 3 & -0.74 & & 61.9 & & \\
\hline 97 & 3 & -0.87 & & 61.3 & & \\
\hline 100 & 4 & 0.22 & 66.7 & & & \\
\hline 102 & 3 & -0.72 & & & 62.0 & \\
\hline 103 & 0 & -2.34 & & & 54.0 & \\
\hline 105 & 2 & -1.11 & & & & 60.1 \\
\hline 108 & 0 & -2.13 & & 55.0 & & \\
\hline 114 & 4 & 0.28 & 67.0 & & & \\
\hline 118 & 1 & 1.57 & & 73.4 & & \\
\hline 119 & 4 & 0.48 & & 68.0 & & \\
\hline 121 & 3 & -0.72 & & & 62.0 & \\
\hline 126 & 1 & 1.89 & 75.0 & & & \\
\hline
\end{tabular}

\begin{tabular}{|c|c|c|c|c|c|c|}
\hline Lab & Rating & Z-value & 1 & 3 & 4 & 6 \\
\hline 127 & 4 & -0.46 & & & 63.3 & \\
\hline 128 & 0 & -2.07 & & & & 55.3 \\
\hline 132 & 3 & 0.89 & & & 70.0 & \\
\hline 133 & 4 & 0.48 & & & 68.0 & \\
\hline 134 & 3 & 0.52 & & 68.2 & & \\
\hline 138 & 4 & -0.28 & & & 64.2 & \\
\hline 140 & 3 & -0.72 & 62.0 & & & \\
\hline 141 & 4 & 0.28 & & & 67.0 & \\
\hline 142 & 2 & -1.17 & & & & 59.8 \\
\hline 145 & 4 & 0.48 & & & 68.0 & \\
\hline 146 & 4 & -0.26 & & & 64.3 & \\
\hline 154 & 3 & -0.93 & & & 61.0 & \\
\hline 180 & 1 & 1.63 & & & 73.7 & \\
\hline 182 & 0 & 2.66 & & & 78.8 & \\
\hline 190 & 4 & -0.30 & & 64.1 & & \\
\hline 191 & 4 & 0.12 & & & & 66.2 \\
\hline 194 & NR & & & & $<100$ & \\
\hline 196 & 4 & -0.14 & & & & 64.9 \\
\hline 198 & 3 & 0.81 & & & 69.6 & \\
\hline 203 & 1 & -1.73 & 57.0 & & & \\
\hline 210 & 3 & 0.99 & & & 70.5 & \\
\hline 212 & 0 & 2.30 & & & 77.0 & \\
\hline 213 & 3 & 0.68 & & 69.0 & & \\
\hline 215 & 4 & -0.12 & & & 65.0 & \\
\hline 219 & 4 & 0.48 & & & & 68.0 \\
\hline 221 & 3 & 0.95 & & 70.3 & & \\
\hline 224 & 2 & -1.31 & & & 59.1 & \\
\hline 231 & 0 & -2.54 & & 53.0 & & \\
\hline 234 & 4 & -0.24 & & 64.4 & & \\
\hline 235 & 4 & -0.32 & & 64.0 & & \\
\hline 236 & 3 & -0.52 & & & 63.0 & \\
\hline 241 & 0 & -6.32 & & 34.2 & & \\
\hline
\end{tabular}

$\begin{aligned} \text { MPV } & =65.6 \\ \text { F-pseudosigma } & =5.0 \\ N= & 80 \\ H u= & 69.0 \\ H I= & 62.3\end{aligned}$


Table 12. Statistical summary of reported data for standard reference water sample T-135 (trace constituents)--Continued $\mathrm{Pb} \quad$ (Lead) $\mu \mathrm{g} / \mathrm{l}$

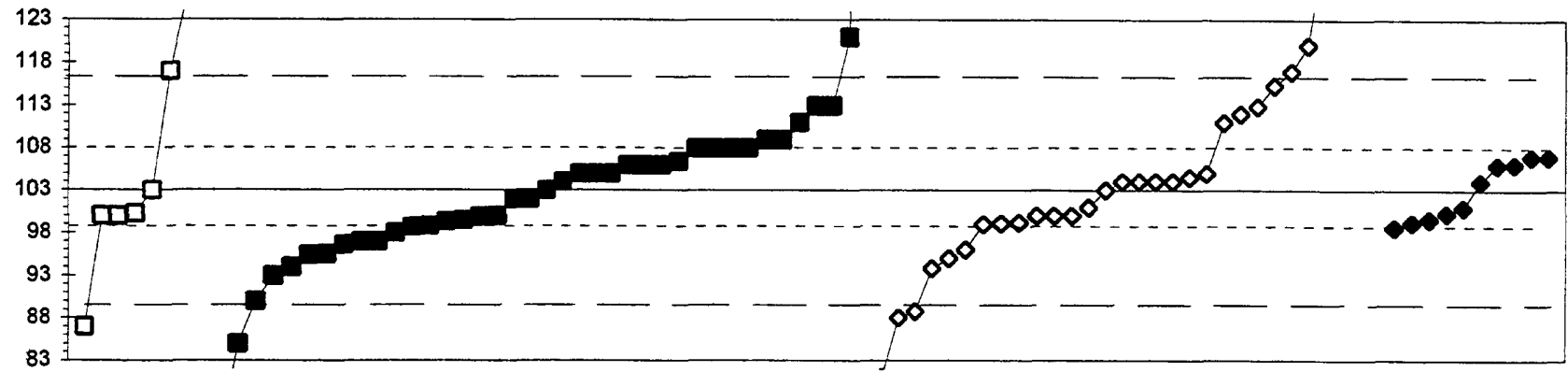

$-0-1-2-0-4-6$

\begin{tabular}{|c|c|c|c|c|c|c|}
\hline \multirow[b]{7}{*}{ Lab } & \multicolumn{3}{|c|}{ 1. AA: direct air } & \multicolumn{3}{|c|}{ 6. ICP/MS } \\
\hline & & $\mathbf{N}=$ & 8 & 39 & 30 & 10 \\
\hline & & imum = & 87 & 74 & 81 & 99 \\
\hline & & imum = & 160 & 187 & 153 & 107 \\
\hline & & edian = & 102 & 103 & 104 & 103 \\
\hline & F-pseud & sigma $=$ & 16 & 8 & 10 & 5 \\
\hline & Rating & $\mathrm{Z}$-value & 1 & 3 & $\overline{4}$ & 6 \\
\hline 1 & 3 & -0.51 & & & & 100 \\
\hline 3 & 4 & 0.30 & & & 105 & \\
\hline 4 & 0 & 4.97 & & & 136 & \\
\hline 7 & 2 & -1.36 & & 94 & & \\
\hline 11 & 0 & 2.56 & & & 120 & \\
\hline 13 & 0 & 2.71 & & 121 & & \\
\hline 15 & 4 & 0.15 & & & 104 & \\
\hline 16 & 0 & -3.32 & & & 81 & \\
\hline 18 & 2 & 1.21 & & 111 & & \\
\hline 19 & 4 & -0.45 & & & 100 & \\
\hline 23 & 4 & 0.30 & & 105 & & \\
\hline 24 & 3 & -0.57 & & & 99 & \\
\hline 25 & 0 & 12.66 & & 187 & & \\
\hline 26 & 3 & 0.75 & & 108 & & \\
\hline 28 & 0 & 7.55 & & & 153 & \\
\hline 30 & 4 & -0.30 & & & & 101 \\
\hline 32 & 3 & -0.57 & & & & 99 \\
\hline 36 & 0 & -2.71 & & 85 & & \\
\hline 39 & 0 & 4.37 & & & 132 & \\
\hline 40 & 0 & 2.11 & & & 117 & \\
\hline 42 & 4 & 0.45 & & & & 106 \\
\hline 46 & 3 & -0.53 & & 100 & & \\
\hline 48 & 0 & 5.58 & & & 140 & \\
\hline 50 & 4 & 0.45 & & 106 & & \\
\hline 52 & 4 & -0.45 & & & 100 & \\
\hline 55 & 3 & 0.90 & & 109 & & \\
\hline 58 & 3 & -0.90 & & 97 & & \\
\hline 59 & 3 & 0.60 & & & & 107 \\
\hline 60 & 4 & -0.41 & 100 & & & \\
\hline 68 & 4 & 0.15 & & 104 & & \\
\hline 69 & 3 & 0.90 & & 109 & & \\
\hline 70 & 4 & -0.47 & & 100 & & \\
\hline 73 & 1 & 1.85 & & & 115 & \\
\hline 75 & 4 & 0.00 & & & 103 & \\
\hline 76 & 4 & 0.00 & & 103 & & \\
\hline 80 & 4 & 0.00 & 103 & & & \\
\hline 81 & 4 & 0.15 & & & 104 & \\
\hline 83 & 4 & -0.15 & & 102 & & \\
\hline 84 & 3 & 0.51 & & 106 & & \\
\hline 85 & 0 & 2.11 & 117 & & & \\
\hline 86 & 3 & -0.59 & & & 99 & \\
\hline 87 & 0 & 3.47 & 126 & & & \\
\hline 89 & 4 & 0.30 & & 105 & & \\
\hline 92 & 4 & -0.45 & 100 & & & \\
\hline 96 & 3 & -0.63 & & 99 & & \\
\hline 97 & 3 & -0.65 & & 99 & & \\
\hline 100 & 1 & 1.51 & & 113 & & \\
\hline 102 & 2 & -1.21 & & & 95 & \\
\hline 103 & 0 & & & & $<30$ & \\
\hline 105 & 4 & 0.15 & & & & 104 \\
\hline
\end{tabular}

\begin{tabular}{|c|c|c|c|c|c|c|}
\hline Lab & Rating & $Z$-value & 1 & 3 & 4 & 6 \\
\hline 107 & 4 & -0.45 & & 100 & & \\
\hline 109 & 0 & -4.41 & & 74 & & \\
\hline 114 & 0 & 8.59 & 160 & & & \\
\hline 118 & 4 & 0.30 & & 105 & & \\
\hline 119 & 4 & 0.45 & & 106 & & \\
\hline 126 & 3 & -0.75 & & 98 & & \\
\hline 127 & 3 & -0.96 & & 97 & & \\
\hline 128 & 4 & -0.41 & & & & 100 \\
\hline 132 & 2 & -1.06 & & & 96 & \\
\hline 133 & 2 & 1.21 & & & 111 & \\
\hline 134 & 4 & 0.23 & & & 105 & \\
\hline 138 & 3 & -0.66 & & & & 99 \\
\hline 140 & 4 & -0.45 & 100 & & & \\
\hline 141 & 0 & -2.26 & & & 88 & \\
\hline 142 & 4 & 0.47 & & & & 106 \\
\hline 145 & 2 & 1.49 & & & 113 & \\
\hline 146 & 4 & -0.30 & & & 101 & \\
\hline 149 & 4 & -0.15 & & 102 & & \\
\hline 154 & 4 & 0.15 & & & 104 & \\
\hline 180 & 2 & 1.36 & & & 112 & \\
\hline 182 & 0 & -2.15 & & & 89 & \\
\hline 190 & 2 & -1.15 & & 95 & & \\
\hline 193 & 1 & 1.51 & & 113 & & \\
\hline 194 & 4 & 0.45 & & 106 & & \\
\hline 196 & 3 & 0.60 & & & & 107 \\
\hline 198 & 3 & 0.75 & & 108 & & \\
\hline 203 & 0 & -2.41 & 87 & & & \\
\hline 210 & 4 & -0.45 & & & 100 & \\
\hline 212 & 1 & -1.51 & & 93 & & \\
\hline 213 & 3 & -0.56 & & 99 & & \\
\hline 215 & 4 & 0.15 & & & 104 & \\
\hline 221 & 3 & 0.75 & & 108 & & \\
\hline 224 & 2 & -1.39 & & & 94 & \\
\hline 231 & 3 & 0.75 & & 108 & & \\
\hline 234 & 2 & -1.13 & & 96 & & \\
\hline 235 & 3 & -0.90 & & 97 & & \\
\hline 236 & 3 & -0.60 & & & 99 & \\
\hline 241 & 1 & -1.96 & & 90 & & \\
\hline
\end{tabular}

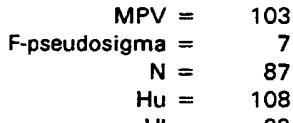

$\mathrm{HI}=99$

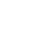


Table 12. Statistical summary of reported data for standard reference water sample T-135 (trace constituents)--Continued $\mathrm{Sb}$ (Antimony) $\mu \mathrm{g} / \mathrm{l}$

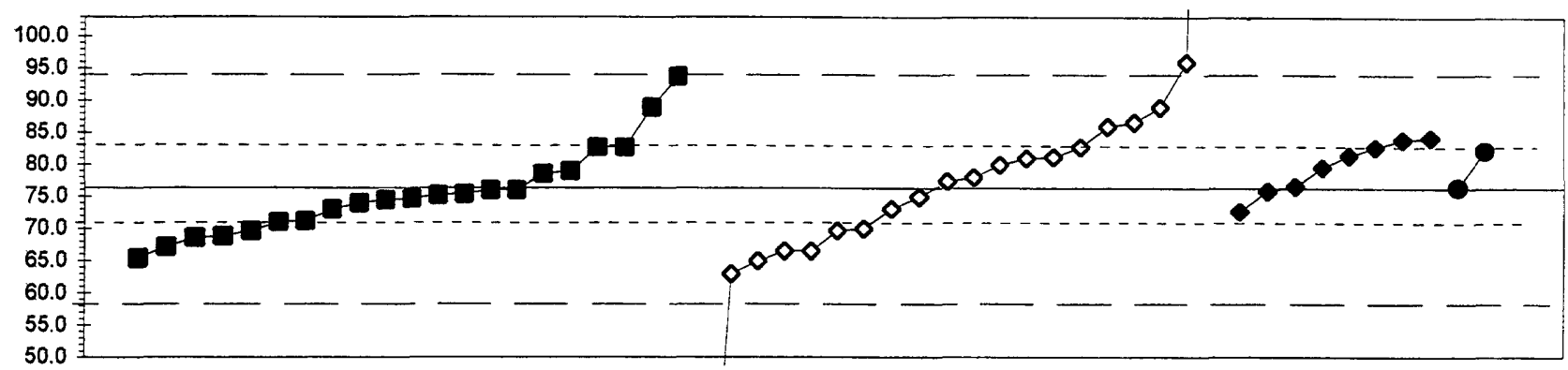

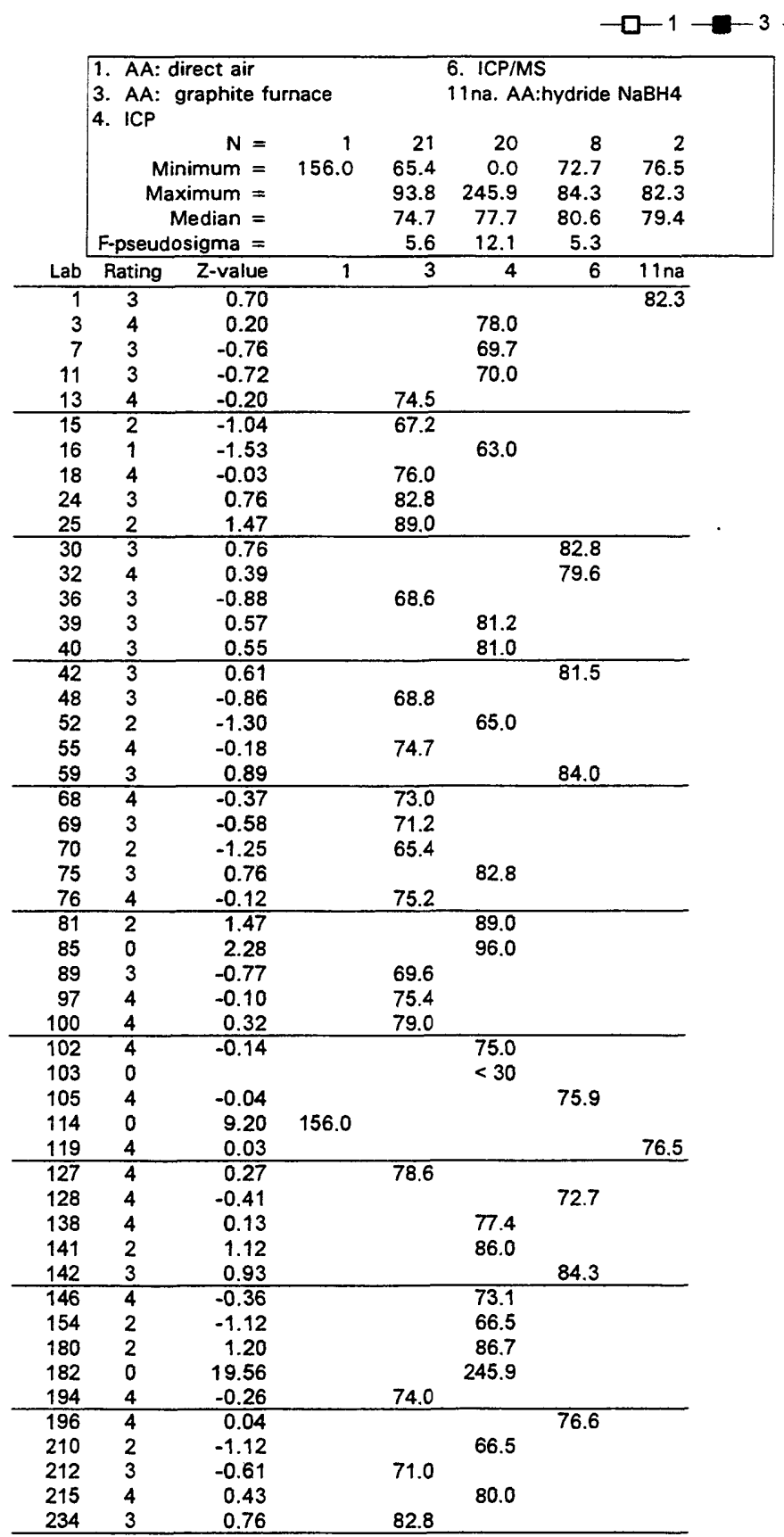

\begin{tabular}{rcrrrrrr} 
Lab & Rating & Z-value & 1 & 3 & 4 & 6 & 11na \\
\hline 235 & 4 & -0.03 & & 76.0 & & & \\
236 & 0 & -8.79 & & & 0.0 & & \\
241 & 1 & 2.02 & & 93.8 & & &
\end{tabular}


Table 12. Statistical summary of reported data for standard reference water sample T-135 (trace constituents)--Continued Se (Selenium)

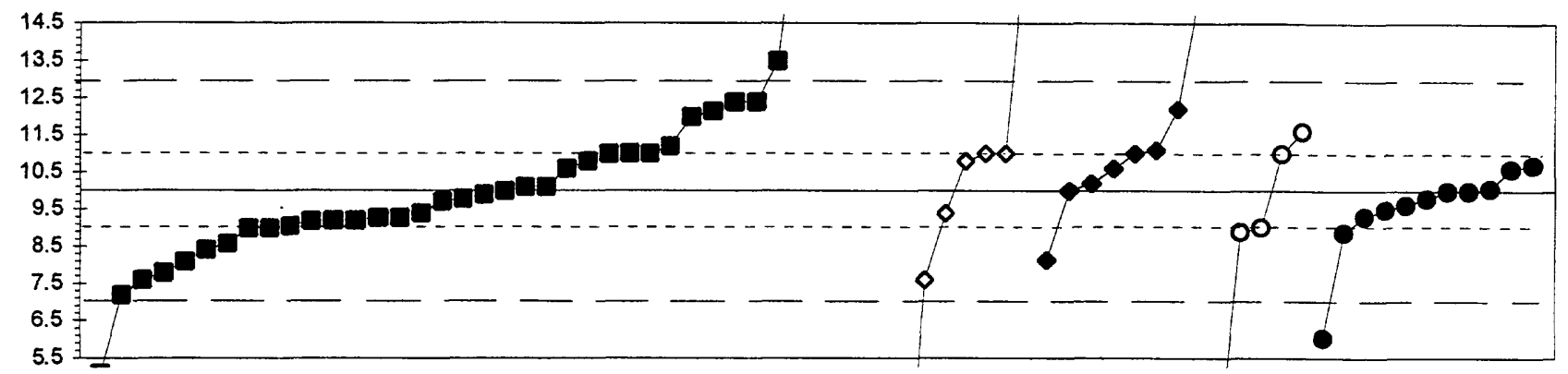

\begin{tabular}{|c|c|c|c|c|c|c|c|c|c|c|c|c|c|c|c|}
\hline \multicolumn{16}{|c|}{$-1-3-0-4-6-0-11-0-11 n a$} \\
\hline & \multicolumn{3}{|c|}{$\begin{array}{l}\text { 3. AA: graphite furnace } \\
\text { 4. ICP } \\
\text { 6. ICP/MS }\end{array}$} & \multicolumn{4}{|c|}{$\begin{array}{l}\text { 11. AA: hydride } \\
\text { 11 na. AA:hydride } \mathrm{NaBH} 4\end{array}$} & & \multirow{6}{*}{\multicolumn{2}{|c|}{$\begin{array}{r}\text { MPV }= \\
\text { F-pseudosigma }= \\
N= \\
H u= \\
H I=\end{array}$}} & \multirow{6}{*}{$\begin{array}{r}10.0 \\
1.4 \\
69 \\
11.0 \\
9.1\end{array}$} & \multirow[b]{7}{*}{4} & \multirow[b]{7}{*}{6} & \multirow[b]{7}{*}{11} & \multirow[b]{7}{*}{$11 \mathrm{na}$} \\
\hline & D. & $\mathbf{N}=$ & 38 & 7 & 8 & 5 & 11 & & & & & & & & \\
\hline & & imum = & 5.1 & 0.0 & 8.1 & 3.0 & 6.0 & & & & & & & & \\
\hline & & imum = & 42.6 & 17.0 & 15.3 & 11.6 & 10.7 & & & & & & & & \\
\hline & & edian = & 10.0 & 10.8 & 10.8 & 9.0 & 9.8 & & & & & & & & \\
\hline & F-pseud & sigma $=$ & 2.2 & 1.9 & 1.1 & & 0.5 & & & & & & & & \\
\hline Lab & Rating & Z-value & 3 & 4 & 6 & 11 & $11 \mathrm{na}$ & Lab & Rating & $Z$-value & 3 & & & & \\
\hline 1 & 4 & 0.07 & 10.1 & & & & & 134 & 4 & 0.42 & & & & & 10.6 \\
\hline 3 & 4 & -0.42 & & 9.4 & & & & 138 & 3 & -0.76 & & & & 8.9 & \\
\hline 7 & 0 & 10.43 & 25.0 & & & & & 141 & 4 & 0.49 & & & & & 10.7 \\
\hline 13 & 1 & -1.67 & 7.6 & & & & & 142 & 2 & -1.29 & & & 8.1 & & \\
\hline 15 & 3 & -0.78 & & & & & 8.9 & 146 & 3 & 0.56 & & 10.8 & & & \\
\hline 16 & NR & & $<70$ & & & & & 151 & 4 & -0.14 & & & & & 9.8 \\
\hline 18 & 2 & 1.11 & & & & 11.6 & & 154 & 3 & -0.70 & 9.0 & & & & \\
\hline 24 & 0 & 22.67 & 42.6 & & & & & 180 & NR & & & $<45.1$ & & & \\
\hline 25 & 3 & -0.56 & 9.2 & & & & & 182 & 4 & 0.05 & & & & & 10.1 \\
\hline 26 & 0 & -2.75 & & & & & 6.0 & 190 & 3 & 0.56 & 10.8 & & & & \\
\hline 28 & 3 & 0.70 & & 11.0 & & & & 191 & 0 & 3.69 & & & 15.3 & & \\
\hline 30 & 1 & 1.53 & & & 12.2 & & & 193 & 4 & -0.42 & 9.4 & & & & \\
\hline 34 & 4 & 0.07 & 10.1 & & & & & 194 & 3 & 0.70 & 11.0 & & & & \\
\hline 35 & 4 & -0.47 & & & & & 9.3 & 196 & 3 & 0.76 & & & 11.1 & & \\
\hline 36 & 3 & -0.65 & 9.1 & & & & & 198 & 3 & $\begin{array}{l}-0.99 \\
\end{array}$ & 8.6 & & & & \\
\hline 39 & 3 & 0.70 & & & & 11.0 & & 203 & 1 & -1.95 & 7.2 & & & & \\
\hline 42 & 3 & 0.70 & & & 11.0 & & & 210 & 3 & 0.70 & & 11.0 & & & \\
\hline 45 & 1 & 1.67 & 12.4 & & & & & 212 & 3 & 0.70 & 11.0 & & & & \\
\hline 46 & 0 & 8.82 & 22.7 & & & & & 215 & 0 & 5.56 & 18.0 & & & & \\
\hline 48 & 3 & -0.70 & 9.0 & & & & & 221 & 4 & .0 .15 & 9.8 & & & & \\
\hline 50 & 4 & 0.00 & & & & & 10.0 & 224 & 1 & -1.67 & & 7.6 & & & \\
\hline 52 & 0 & & & $<5$ & & & & 231 & 2 & -1.10 & 8.4 & & & & \\
\hline 55 & 4 & -0.07 & 9.9 & & & & & 234 & 3 & -0.56 & 9.2 & & & & \\
\hline 58 & 0 & -4.87 & & & & 3.0 & & 235 & 3 & -0.56 & 9.2 & & & & \\
\hline 59 & 4 & 0.00 & & & 10.0 & & & 236 & NR & -6.95 & & 0.0 & & & \\
\hline 60 & 3 & 0.83 & 11.2 & & & & & 241 & 0 & 2.43 & 13.5 & & & & \\
\hline 68 & 4 & -0.49 & 9.3 & & & & & & & & & & & & \\
\hline 69 & 4 & 0.42 & 10.6 & & & & & & & & & & & & \\
\hline 70 & 3 & 0.70 & 11.0 & & & & & & & & & & & & \\
\hline 75 & 4 & -0.26 & & & & & 9.6 & & & & & & & & \\
\hline 80 & 2 & 1.39 & 12.0 & & & & & & & & & & & & \\
\hline 81 & 4 & 0.00 & 10.0 & & & & & & & & & & & & \\
\hline 85 & 4 & -0.35 & & & & & 9.5 & & & & & & & & \\
\hline 86 & 3 & -0.67 & & & & 9.0 & & & & & & & & & \\
\hline 87 & 0 & & & & & $<2$ & & & & & & & & & \\
\hline 89 & 0 & & & & & & $\overline{<2}$ & & & & & & & & \\
\hline 96 & 4 & -0.20 & 9.7 & & & & & & & & & & & & \\
\hline 97 & 0 & 5.98 & 18.6 & & & & & & & & & & & & \\
\hline 100 & 1 & -1.53 & 7.8 & & & & & & & & & & & & \\
\hline 102 & 0 & 4.87 & & 17.0 & & & & & & & & & & & \\
\hline 103 & NR & & & $<30$ & & & & & & & & & & & \\
\hline 105 & 4 & 0.42 & & & 10.6 & & & & & & & & & & \\
\hline 108 & 2 & -1.32 & 8.1 & & & & & & & & & & & & \\
\hline 109 & 2 & 1.50 & 12.2 & & & & & & & & & & & & \\
\hline 118 & 0 & -3.41 & 5.1 & & & & & & & & & & & & \\
\hline 119 & 4 & 0.00 & & & & & 10.0 & & & & & & & & \\
\hline 126 & 0 & & & & & & $<1$ & & & & & & & & \\
\hline 127 & 4 & -0.50 & 9.3 & & & & & & & & & & & & \\
\hline 128 & 4 & 0.14 & & & 10.2 & & & & & & & & & & \\
\hline 133 & 1 & 1.67 & 12.4 & & & & & & & & & & & & \\
\hline
\end{tabular}


Table 12. Statistical summary of reported data for standard reference water sample T-135 (trace constituents)--Continued $\mathrm{SiO2}$ (Silica) $\mathrm{mg} / \mathrm{l}$

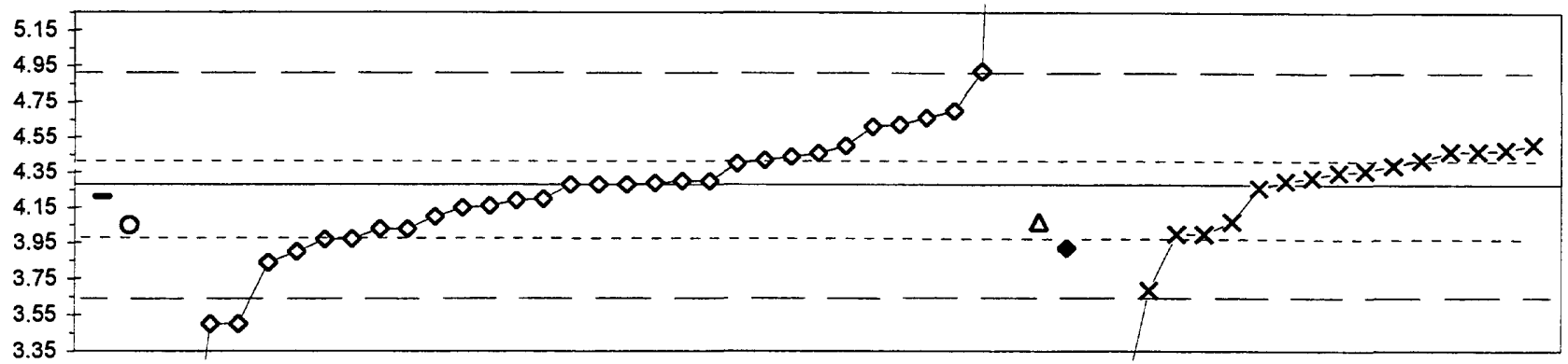

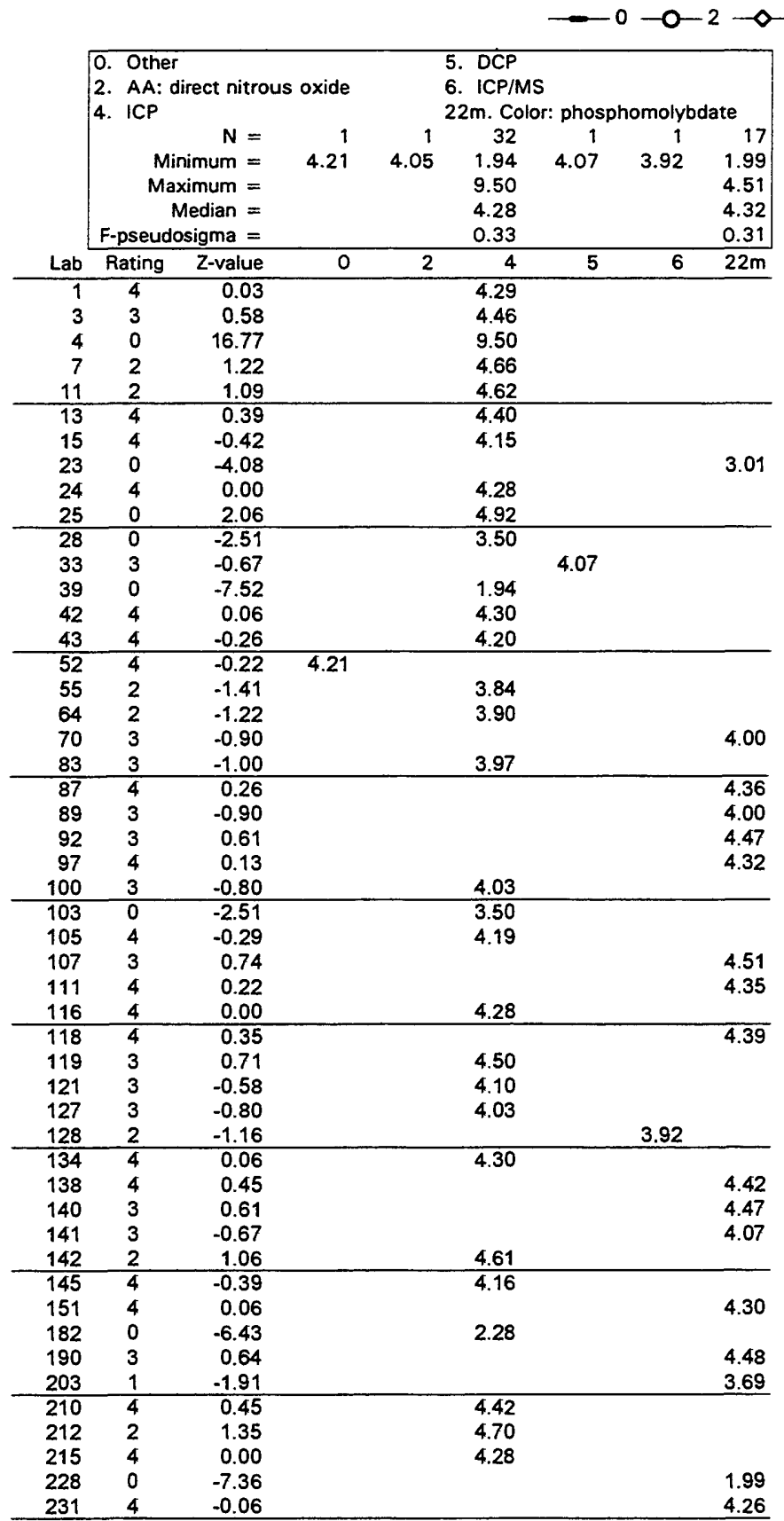


Table 12. Statistical summary of reported data for standard reference water sample T-135 (trace constituents)--Continued Sr (Strontium) $\mu \mathrm{g} / \mathrm{l}$

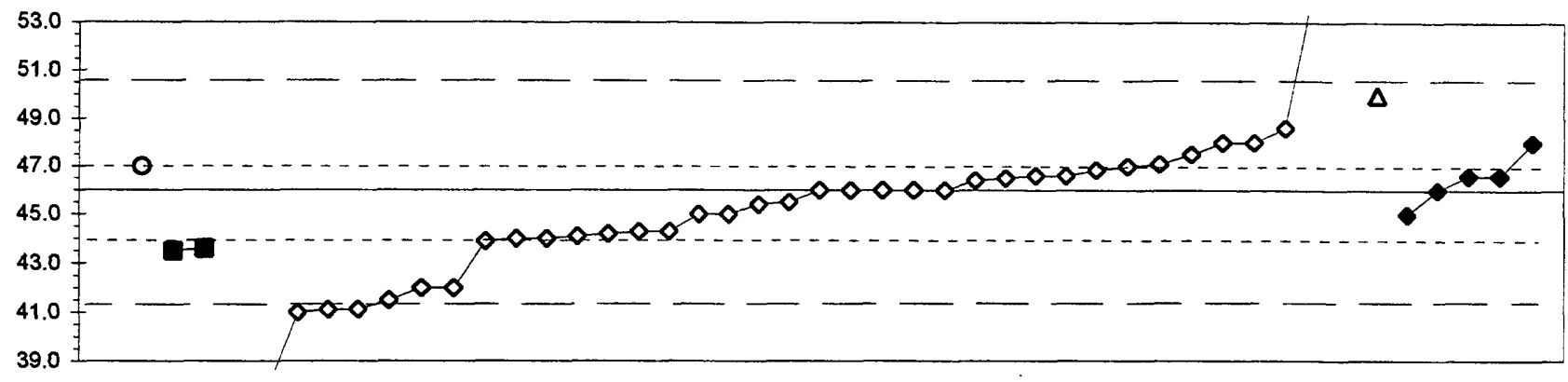

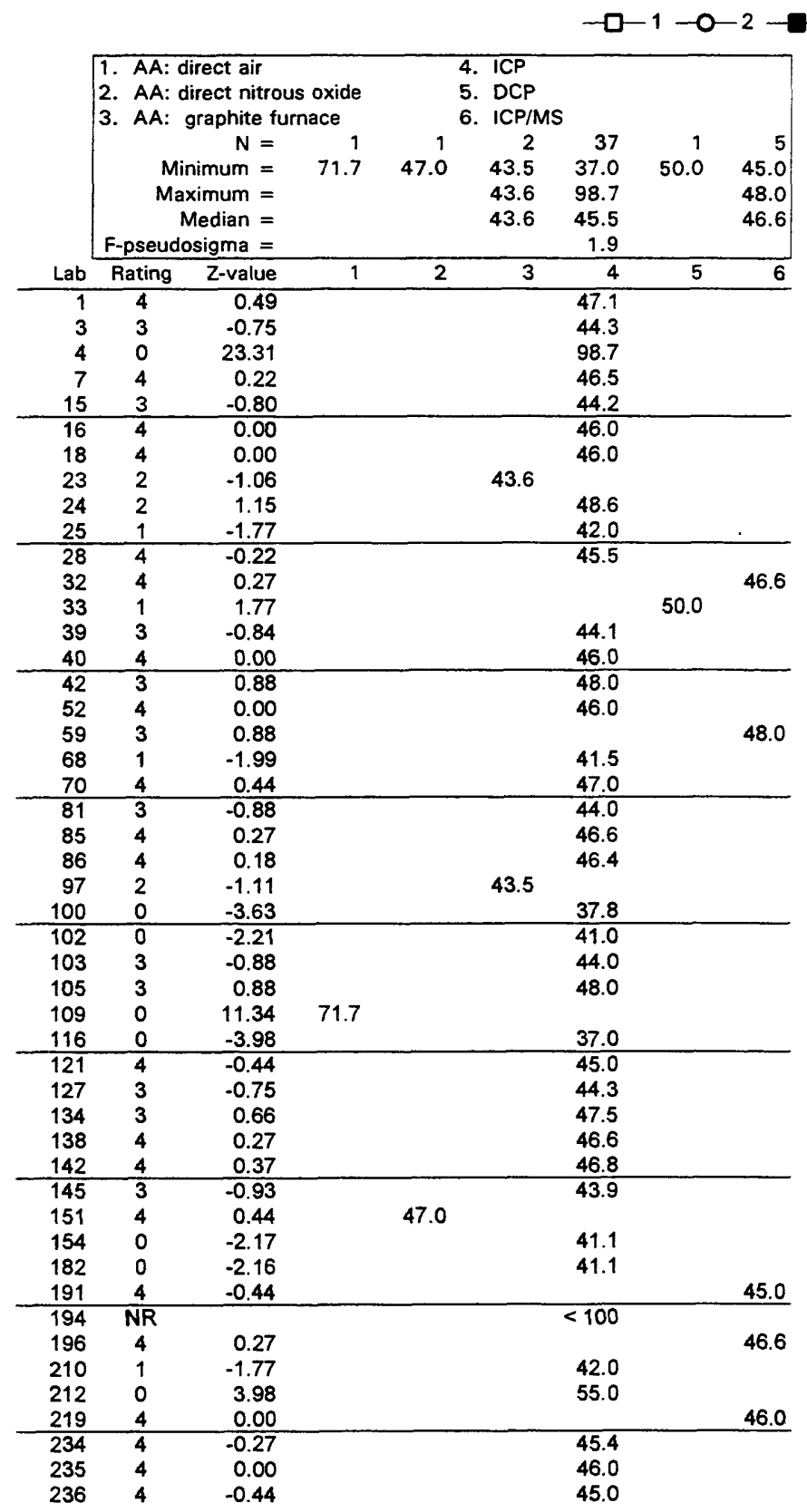


Table 12. Statistical summary of reported data for standard reference water sample T-135 (trace constituents)--Continued V (Vanadium) $\mu \mathrm{g} / \mathrm{l}$
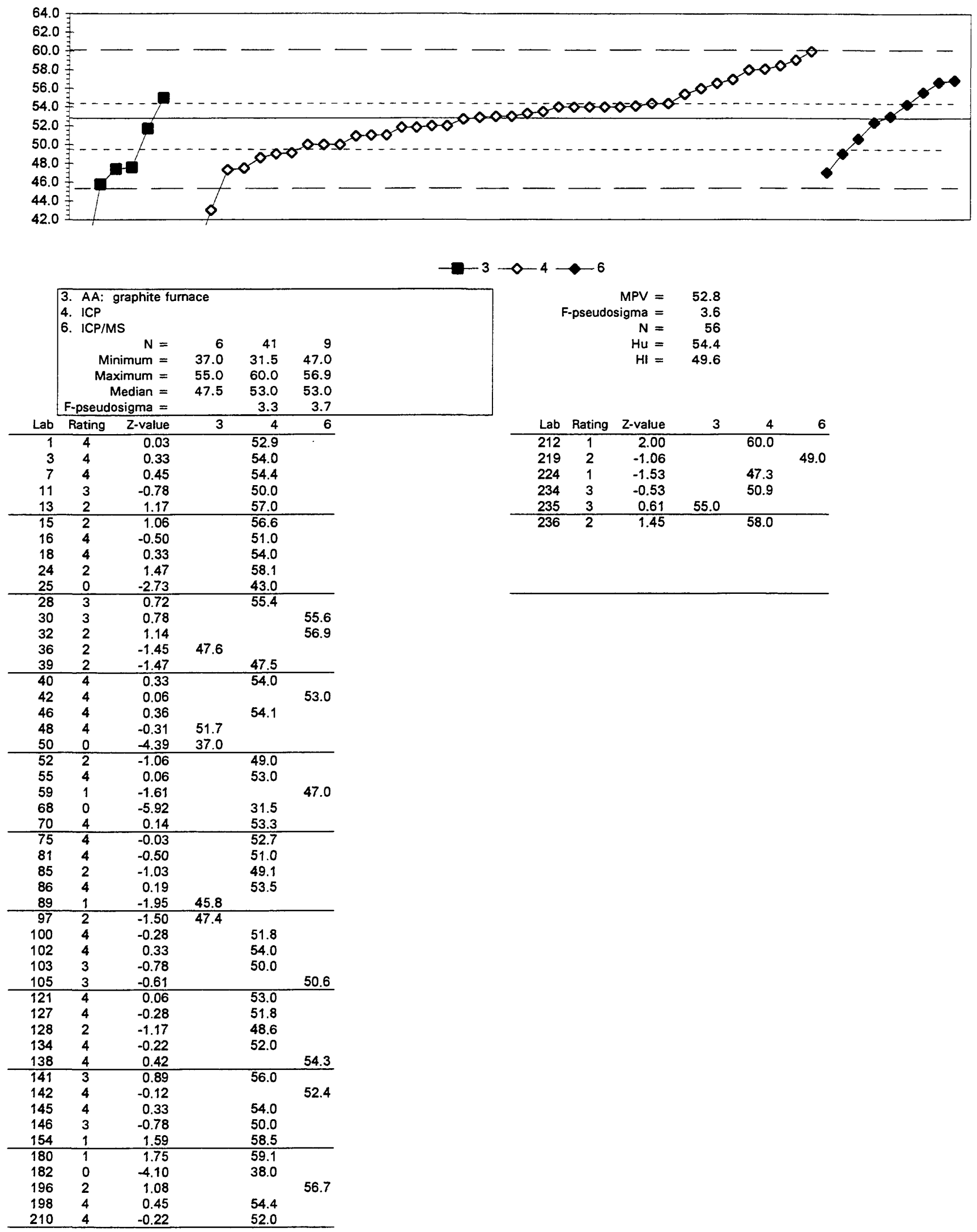
Table 12. Statistical summary of reported data for standard reference water sample T-135 (trace constituents)--Continued Zn (Zinc) $\mu \mathrm{g} / \mathrm{l}$

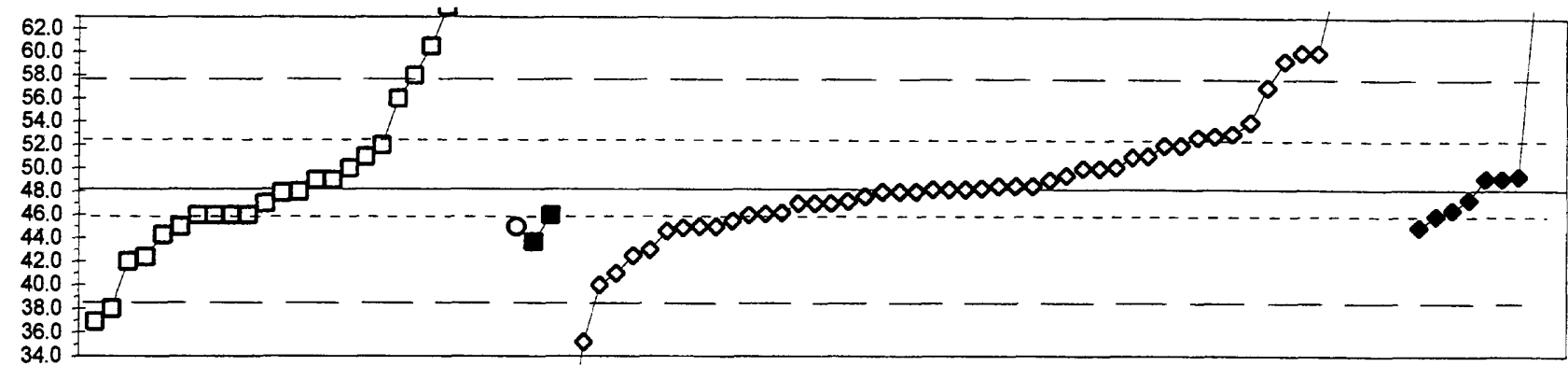

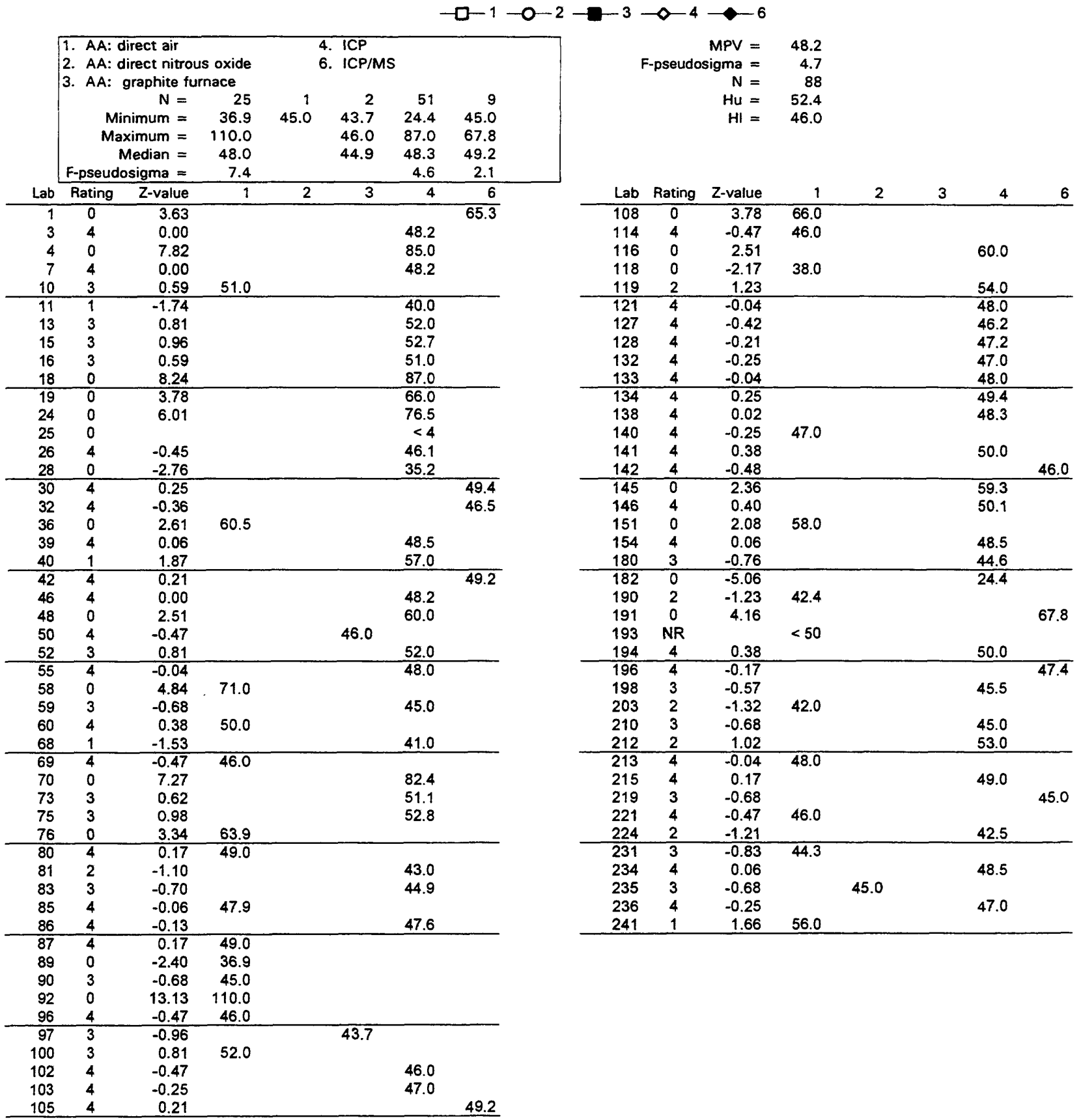


Table 13. Statistical summary of reported data for standard reference water sample M-134 (major constituents)

Definition of analytical methods, abbreviations, and symbols

\begin{tabular}{l} 
Analytical methods \\
\hline 0 Other/Not reported \\
1 AA: direct, air \\
2 AA: direct, N2O \\
3 AA: graphite fumace \\
4 ICP \\
5 DCP \\
6 ICP/MS \\
7 IC \\
12 Flame emission \\
20 Titrate: color \\
21 Titrate: electro \\
22 Color: \\
40 lon electrode \\
41 Electro \\
50 Gravimetric \\
51 Turbidimetric
\end{tabular}

atomic absorption: direct, air

= atomic absorption: direct, nitrous oxide

= atomic absorption: graphite fumace

= inductively coupled plasma

= direct current plasma

= mass spectrometry/inductively coupled plasma

$=$ ion chromatography

$=$ flame emission

$=$ titration: colorimetric [color reagent specified]

$=$ titration: electrometric

$=$ colorimetric [color reagent specified]

$=$ ion selective electrode

$=$ electrometric: [type meter specified]

= gravimetric: [precipitate specified]

51 Turbidimetric

$=$ turbidimetric: $[$ recipitate specified]

Abbreviations and symbols

\begin{tabular}{|c|c|}
\hline$N=$ & number of samples \\
\hline St dev $=$ & traditional standard deviation \\
\hline$M P V=$ & most probable value \\
\hline F-pseudosigma $=$ & nonparametric statistic deviation \\
\hline $\mathrm{Hu}=$ & upper hinge value \\
\hline $\mathrm{HI}=$ & lower hinge value \\
\hline$\mu g / L=$ & micrograms per liter \\
\hline $\mathrm{mg} / \mathrm{L}=$ & milligrams per liter \\
\hline$\mu \mathrm{S} / \mathrm{cm}=$ & microsiemens per centimeter at $25 \mathrm{C}$ \\
\hline$L a b=$ & laboratory code number \\
\hline $\begin{aligned} & N R= \\
&<=\end{aligned}$ & $\begin{array}{l}\text { not rated, less than value reported } \\
\text { less than }\end{array}$ \\
\hline
\end{tabular}

Constituent

Alk Alkalinity as $\mathrm{CaCO} 3$

page

B Boron

66

Ca Calcium

67

$\mathrm{Cl}$ Chloride

68

DSRD Dissolved solids $\quad 70$

69

F Fluoride $\quad 71$

K Potassium 72

$\mathrm{Mg}$ Magnesium $\quad 73$

$\mathrm{Na}$ Sodium $\quad 74$

total P Phosphorus $\quad 75$

$\mathrm{pH} \quad 76$

$\mathrm{SiO} 2$ Silica $\quad 77$

$\mathrm{SO} 4$ Sulfate 78

Sp Co Specific Conductance $\quad 79$

$\mathrm{Sr}$ Strontium $\quad 80$

$\checkmark$ Vanadium $\quad 81$ 
Table 13. Statistical summary of reported data for standard reference water sample M-134 (major constituents)--Continued Alk (Alkalinity as calcium carbonate) $\mathrm{mg} / \mathrm{l}$

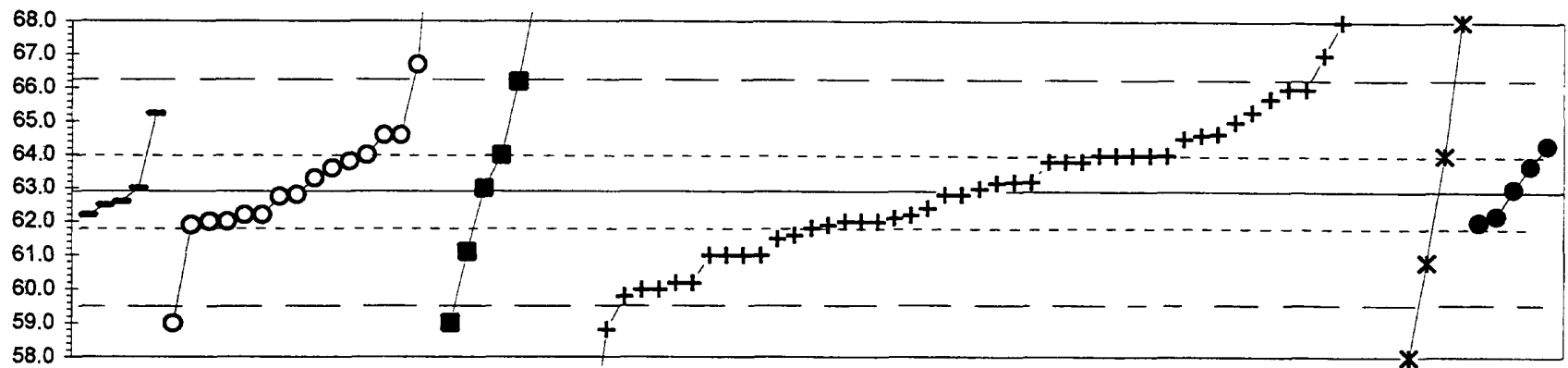

\begin{tabular}{|c|c|c|c|c|c|c|c|c|}
\hline \multirow[b]{6}{*}{ Lab } & \multicolumn{4}{|c|}{$\begin{array}{l}0 . \text { Other } \\
20 \text {. Titrate: colorimetric } \\
20 \text { br. Titrate: color brom cresol gre }\end{array}$} & \multicolumn{4}{|c|}{$\begin{array}{l}\text { 21. Titrate: electrometric } \\
\text { 22. Colorimetric } \\
\text { 41. Direct reading }\end{array}$} \\
\hline & & $N=$ & 5 & 16 & 6 & 49 & 5 & 5 \\
\hline & & imum = & 62.2 & 59.0 & 59.0 & 0.1 & 55.0 & 62.0 \\
\hline & $\mathrm{Ma}$ & imum = & 65.2 & 72.8 & 69.0 & 75.4 & 68.0 & 64.3 \\
\hline & F-pseud & $\begin{array}{l}\text { ledian }= \\
\text { sigma }=\end{array}$ & 62.6 & $\begin{array}{r}63.1 \\
1.6 \\
\end{array}$ & 63.5 & $\begin{array}{r}62.8 \\
2.2\end{array}$ & 60.8 & 63.0 \\
\hline & Rating & Z-value & 0 & 20 & $20 \mathrm{br}$ & 21 & 22 & 41 \\
\hline 1 & 2 & 1.07 & & & & 64.6 & & \\
\hline 3 & 0 & 6.07 & & 72.8 & & & & \\
\hline 9 & 2 & 1.04 & & & & 64.6 & & \\
\hline 10 & 2 & 1.04 & & 64.6 & & & & \\
\hline 11 & 4 & -0.43 & & & & & & 62.2 \\
\hline 13 & 1 & -1.66 & & & & 60.2 & & \\
\hline 15 & 0 & -2.39 & & 59.0 & & & & \\
\hline 16 & 4 & -0.09 & & 62.8 & & & & \\
\hline 18 & 2 & -1.29 & & & & & 60.8 & \\
\hline 19 & 1 & 1.72 & & & & 65.7 & & \\
\hline 23 & 3 & -0.61 & & & & 61.9 & & \\
\hline 24 & 1 & -1.90 & & & & 59.8 & & \\
\hline 25 & 0 & 2.51 & & & & 67.0 & & \\
\hline 26 & 3 & 0.98 & & & & 64.5 & & \\
\hline 28 & 0 & 2.33 & & 66.7 & & & & \\
\hline 32 & 4 & -0.43 & 62.2 & & & & & \\
\hline 33 & 4 & -0.49 & & & & 62.1 & & \\
\hline 36 & 0 & 3.13 & & & & 68.0 & & \\
\hline 39 & 0 & 4.35 & & & & 70.0 & & \\
\hline 40 & 3 & 0.55 & & 63.8 & & & & \\
\hline 42 & 3 & -0.67 & & & & 61.8 & & \\
\hline 43 & 3 & 0.67 & & & & 64.0 & & \\
\hline 46 & 4 & -0.06 & & & & 62.8 & & \\
\hline 48 & 0 & -3.00 & & & & & 58.0 & \\
\hline 50 & 2 & -1.17 & & & & 61.0 & & \\
\hline 52 & 4 & 0.06 & 63.0 & & & & & \\
\hline 54 & 1 & -1.78 & & & & 60.0 & & \\
\hline 55 & 1 & 1.90 & & & & 66.0 & & \\
\hline 56 & 2 & 1.43 & 65.2 & & & & & \\
\hline 57 & 3 & -0.55 & & 62.0 & & & & \\
\hline 58 & 2 & -1.17 & & & & 61.0 & & \\
\hline 59 & 3 & -0.55 & & & & 62.0 & & \\
\hline 68 & 0 & 3.13 & & & & & 68.0 & \\
\hline 69 & 3 & 0.67 & & & & & 64.0 & \\
\hline 70 & 3 & -0.55 & & & & & & 62.0 \\
\hline 75 & 4 & 0.49 & & & & & & 63.7 \\
\hline 76 & 2 & -1.10 & & & 61.1 & & & \\
\hline 80 & 3 & 0.67 & & 64.0 & & & & \\
\hline 81 & 4 & -0.43 & & 62.2 & & & & \\
\hline 83 & 4 & -0.25 & 62.5 & & & & & \\
\hline 84 & 0 & -5.09 & & & & 54.6 & & \\
\hline 85 & 3 & -0.86 & & & & 61.5 & & \\
\hline 89 & 4 & -0.06 & & 62.8 & & & & \\
\hline 90 & 4 & 0.06 & & & 63.0 & & & \\
\hline 92 & 4 & -0.06 & & & & 62.8 & & \\
\hline 96 & 2 & 1.29 & & & & 65.0 & & \\
\hline 97 & 3 & 0.55 & & & & 63.8 & & \\
\hline 100 & 2 & 1.04 & & 64.6 & & & & \\
\hline 105 & 4 & 0.06 & & & & 63.0 & & \\
\hline 107 & 1 & -1.66 & & & & 60.2 & & \\
\hline 109 & 0 & 7.69 & & & & 75.4 & & \\
\hline 111 & 4 & 0.25 & & 63.3 & & & & \\
\hline 114 & 3 & 0.67 & & & & 64.0 & & \\
\hline 118 & 3 & 0.67 & & & 64.0 & & & \\
\hline 119 & 3 & -0.55 & & 62.0 & & & & \\
\hline
\end{tabular}

$20 \mathrm{br}+21-x-22-41$

\begin{tabular}{rrrllllll} 
Lab & Rating & Z-value & 0 & 20 & 20 br & 21 & 22 & 41 \\
\hline 127 & 3 & 0.55 & & & & 63.8 & & \\
128 & 4 & 0.18 & & & & 63.2 & & \\
129 & 0 & 3.74 & & & 69.0 & & &
\end{tabular}

$+136$

$132 \quad 0 \quad-38.53$

\begin{tabular}{lllll}
134 & 3 & 0.87 & 65.3 & 64.3 \\
\hline 136 & 2 & 1.47 & 64.0
\end{tabular}

$\begin{array}{llll}138 & 3 & 0.67 & 65.3 \\ 141 & 3 & 0.55 & 64.0\end{array}$

$\begin{array}{llll}141 & 3 & 0.55 & 63.8\end{array}$

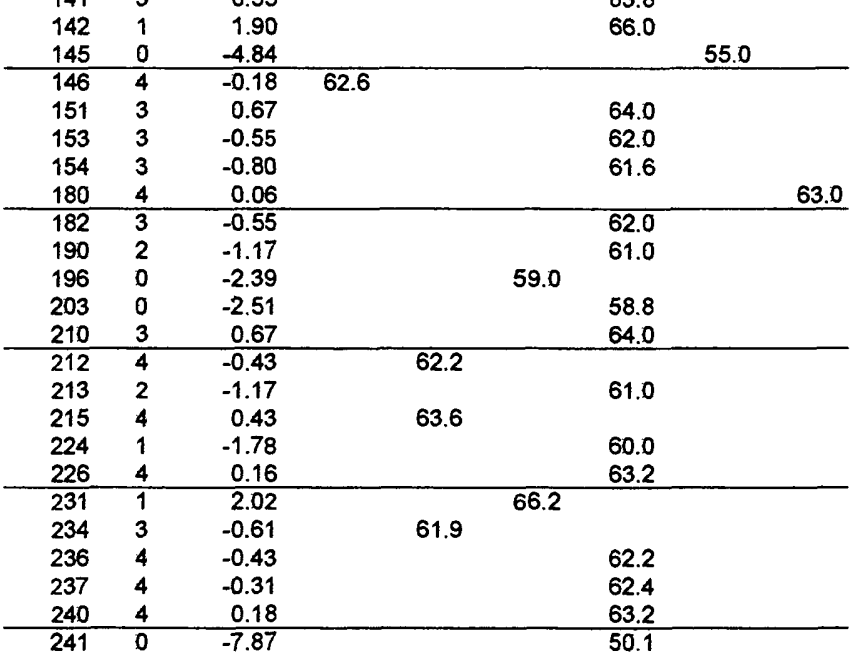
.0

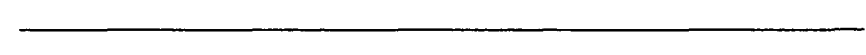


Table 13. Statistical summary of reported data for standard reference water sample $M$ - 134 (major constituents)--Continued B (Boron)

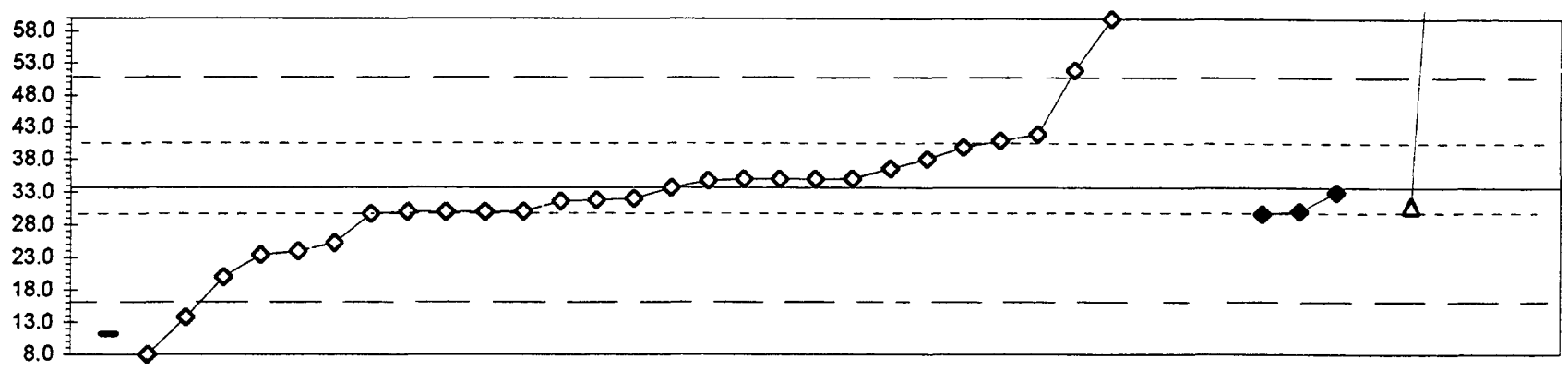

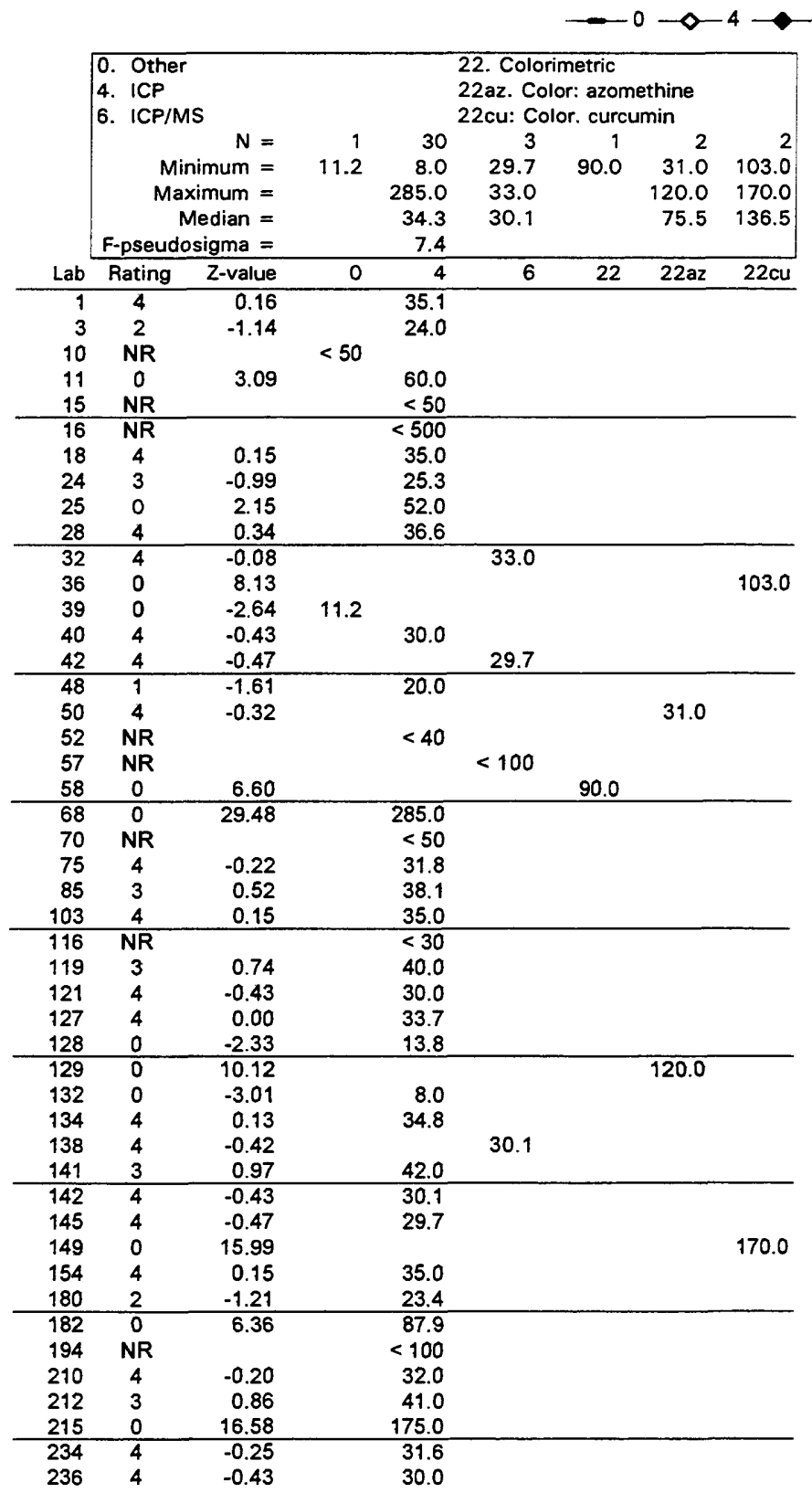


Table 13. Statistical summary of reported data for standard reference water sample $M-134$ (major constituents)--Continued $\mathrm{Ca}$ (Calcium) $\mathrm{mg} / \mathrm{l}$

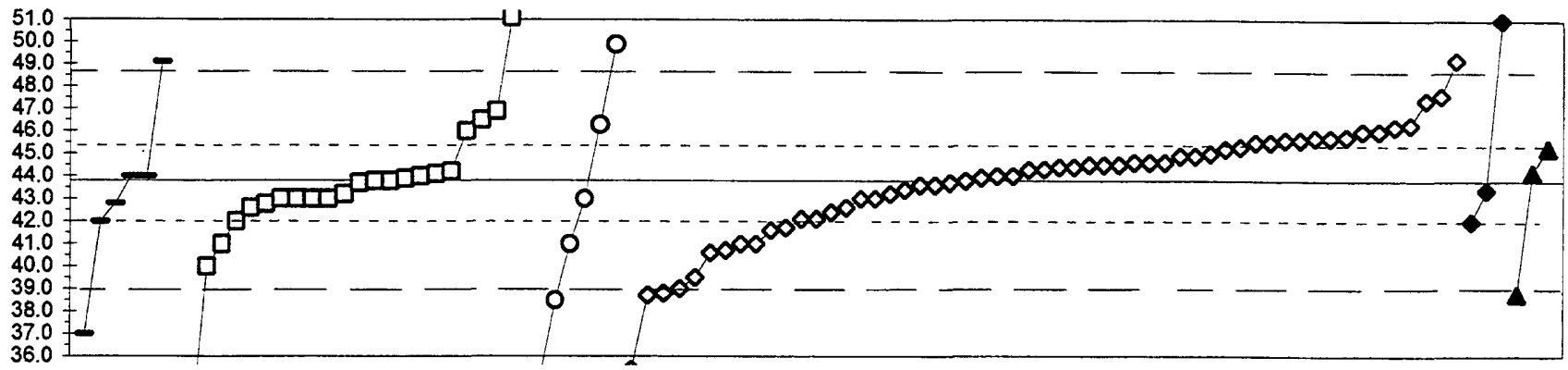

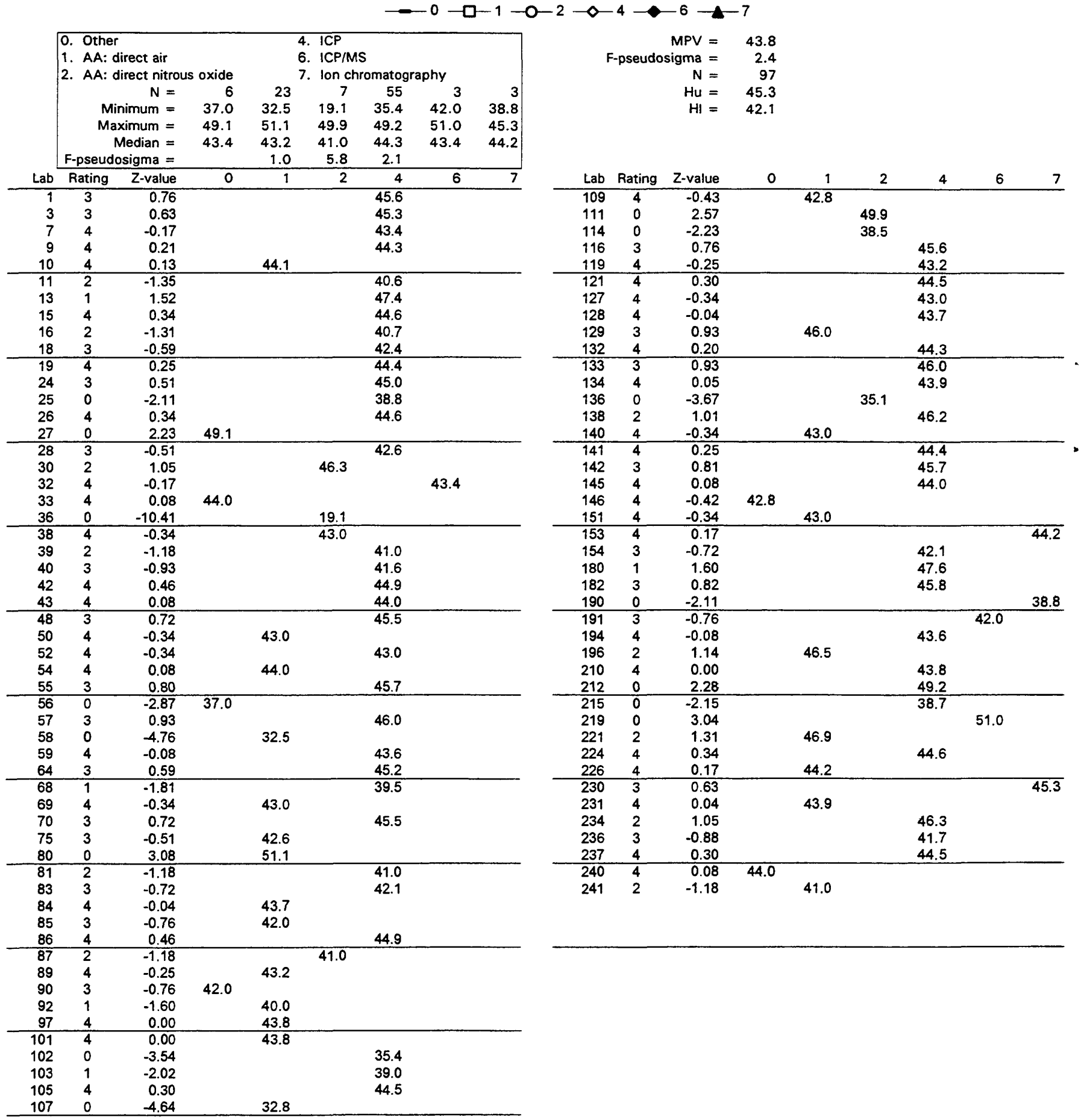


Table 13. Statistical summary of reported data for standard reference water sample M-134 (major constituents)--Continued Cl (Chloride)

$\mathrm{mg} / \mathrm{l}$

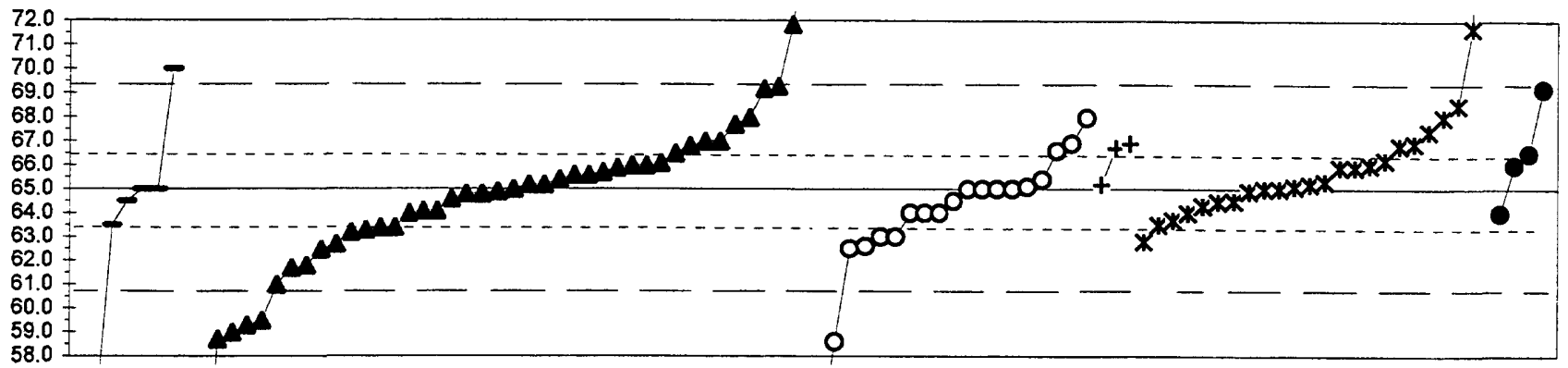

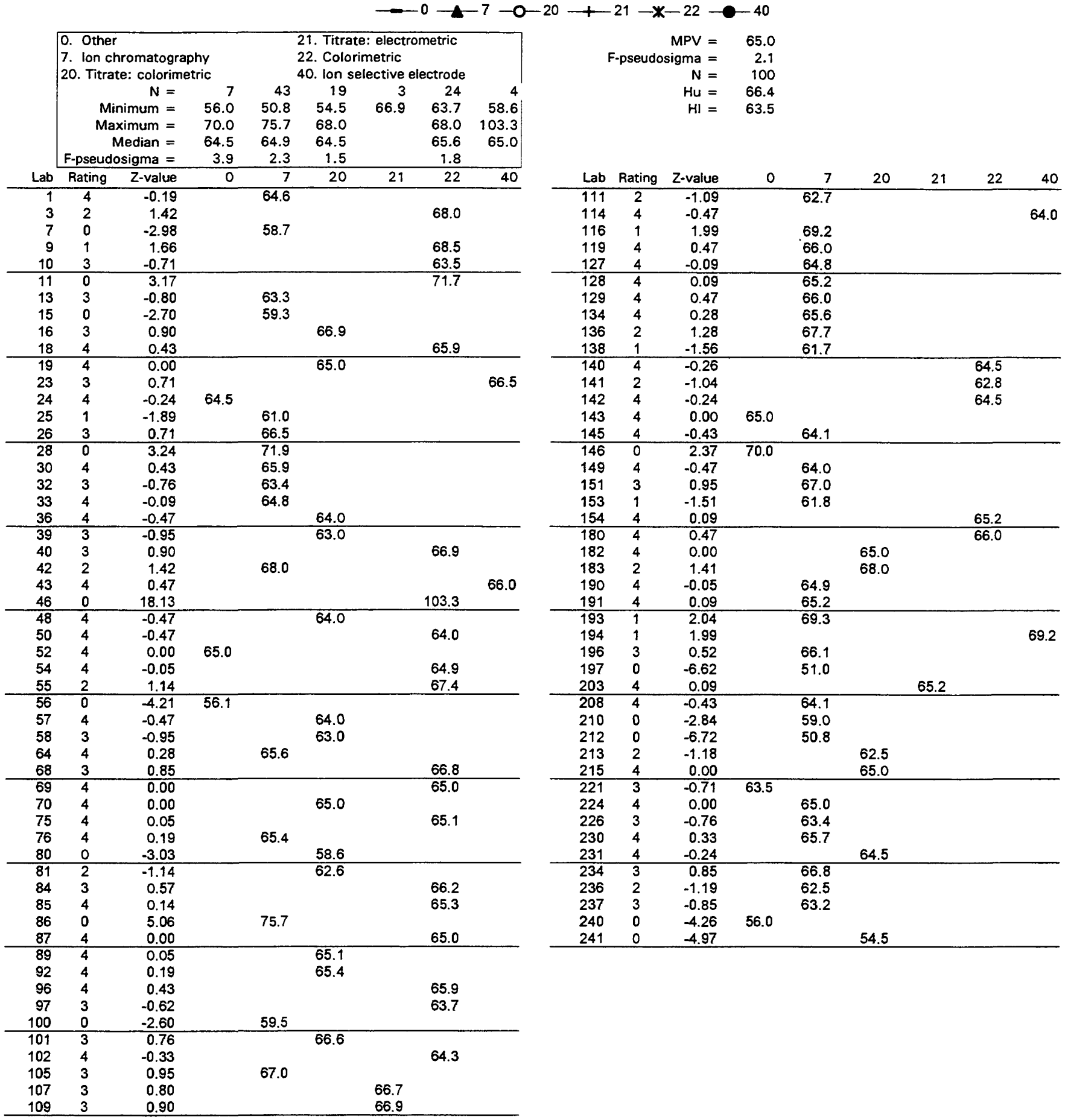


Table 13. Statistical summary of reported data for standard reference water sample M-134 (major constituents)--Continued DSRD (Dissolved solids) $\mathrm{mg} / \mathrm{l}$

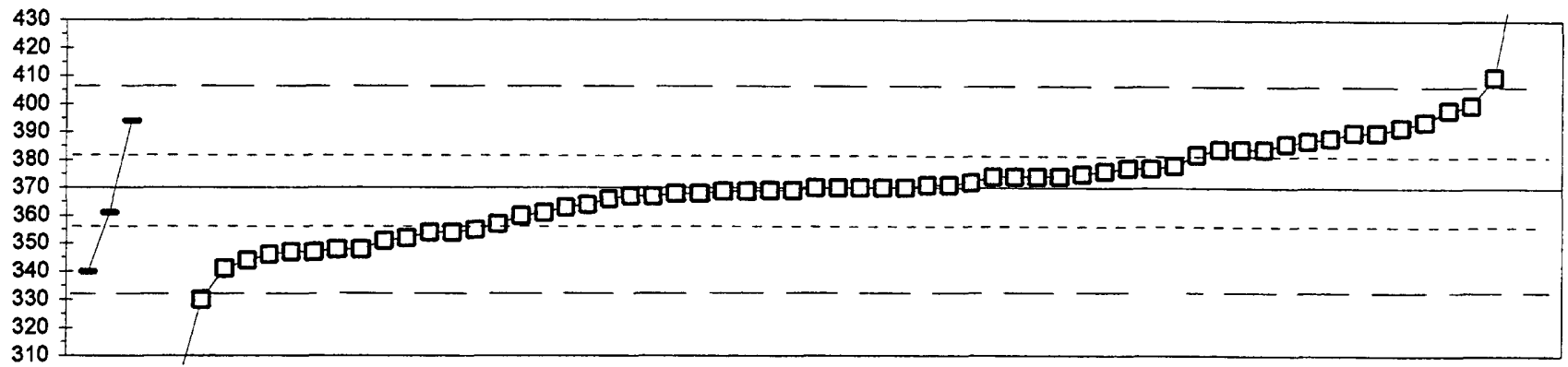

$-0 \rightarrow-50$

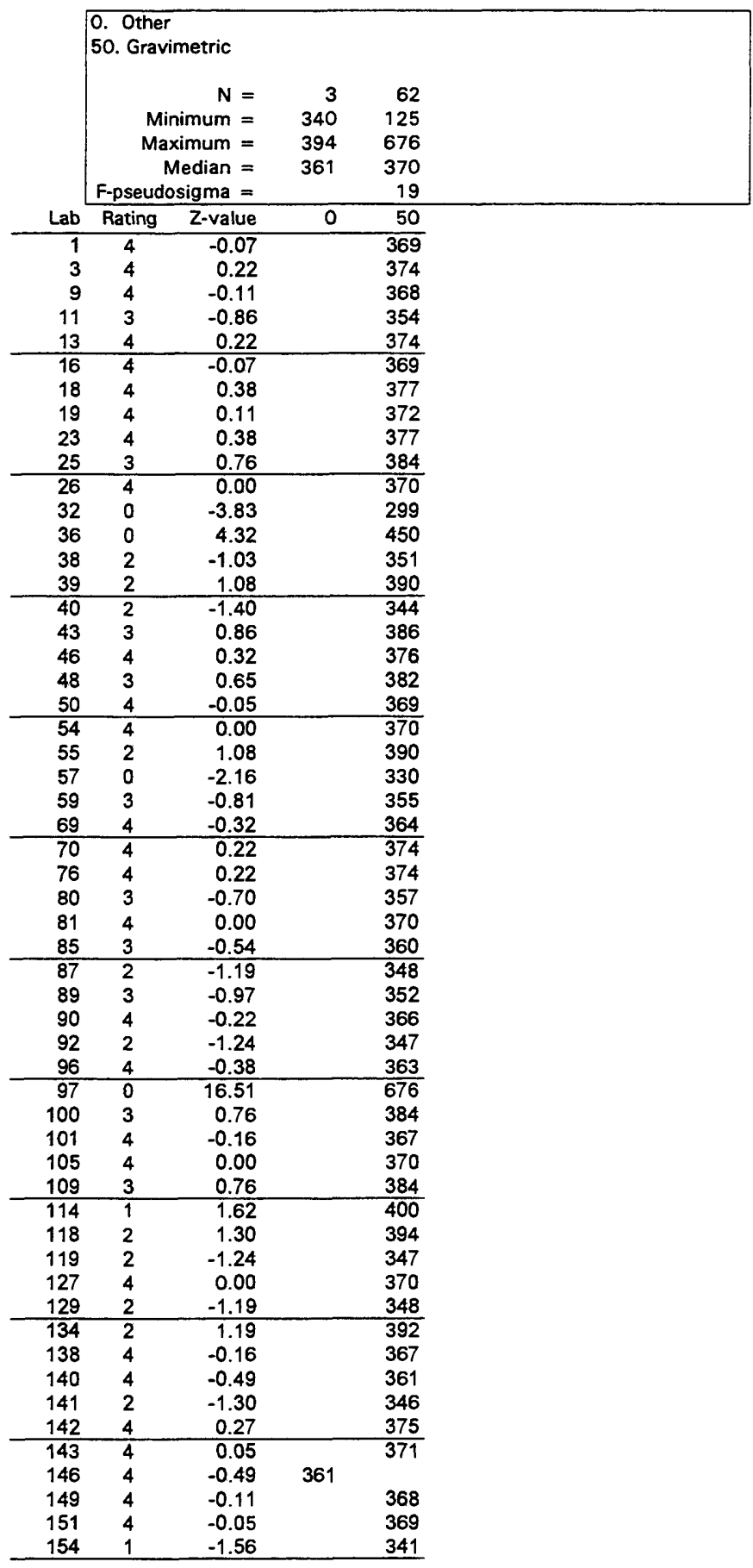

\begin{tabular}{rrrrr} 
Lab & Rating & Z-value & 0 & 50 \\
\hline 182 & 3 & 0.92 & & 387 \\
190 & 0 & 2.16 & & 410 \\
194 & 3 & -0.86 & & 354 \\
212 & 4 & 0.05 & & 371 \\
215 & 4 & 0.43 & & 378 \\
\hline 221 & 2 & 1.30 & 394 & \\
224 & 1 & 1.52 & & 398 \\
234 & 3 & 0.97 & & 388 \\
236 & 0 & -13.22 & & 125 \\
240 & 1 & -1.62 & 340 & \\
\hline
\end{tabular}


Table 13. Statistical summary of reported data for standard reference water sample M-134 (major constituents)--Continued $F$ (Fluoride)

$\mathrm{mg} / \mathrm{l}$

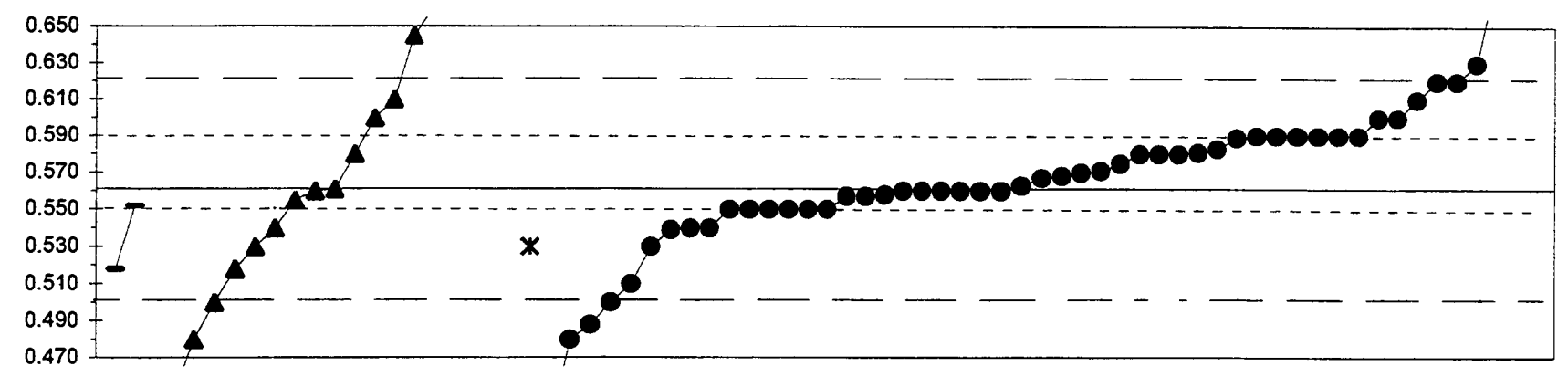

\begin{tabular}{|c|c|c|c|c|c|c|c|c|c|c|c|c|c|}
\hline \multirow[b]{2}{*}{ Lab } & $\begin{array}{l}\text { 0. Othe } \\
\text { 7. Ion } \\
\text { 22. Colc } \\
\text { F-pseud }\end{array}$ & $\begin{array}{r}\text { romatogr } \\
\text { imetric } \\
N= \\
\text { imum }= \\
\text { imum }= \\
\text { ledian }= \\
\text { sigma }=\end{array}$ & $\begin{array}{r}\text { phy } \\
2 \\
2 \\
0.518 \\
0.552 \\
0.535\end{array}$ & $\begin{array}{r}19 \\
0.270 \\
0.850 \\
0.561 \\
0.095 \\
\end{array}$ & $\begin{array}{r}1 \\
0.530\end{array}$ & $\begin{array}{r}51 \\
0.430 \\
0.980 \\
0.567 \\
0.030 \\
\end{array}$ & & F-pseudc & $\begin{aligned} M P V & = \\
\text { sigma } & = \\
N & = \\
H u & = \\
H I & =\end{aligned}$ & $\begin{array}{r}0.561 \\
0.030 \\
73 \\
0.590 \\
0.550\end{array}$ & \multirow[b]{2}{*}{7} & \multirow[b]{2}{*}{22} & \multirow[b]{2}{*}{40} \\
\hline & Rating & $\mathrm{Z}$-value & 0 & 7 & 22 & 40 & Lab & Rating & $Z$-value & 0 & & & \\
\hline 1 & 1 & 1.99 & & & & 0.620 & 142 & 3 & 0.98 & & & & 0.590 \\
\hline 3 & 0 & 4.38 & & & & 0.691 & 145 & 0 & 3.34 & & 0.660 & & \\
\hline 4 & 0 & 4.69 & & 0.700 & & & 146 & 4 & -0.30 & 0.552 & & & \\
\hline 7 & 0 & 9.75 & & 0.850 & & & 149 & 2 & 1.32 & & 0.600 & & \\
\hline 9 & 4 & -0.10 & & & & 0.558 & 153 & 0 & -2.73 & & 0.480 & & \\
\hline 10 & 3 & 0.98 & & & & 0.590 & 154 & 2 & -1.05 & & & 0.530 & \\
\hline 11 & 3 & 0.67 & & & & 0.581 & 180 & 4 & -0.37 & & & & 0.550 \\
\hline 13 & 0 & 2.33 & & & & 0.630 & 182 & 0 & -2.46 & & & & 0.488 \\
\hline 15 & 3 & 0.94 & & & & 0.589 & 190 & 4 & 0.20 & & & & 0.567 \\
\hline 16 & 4 & -0.37 & & & & 0.550 & 196 & 4 & 0.07 & & & & 0.563 \\
\hline 18 & 4 & -0.13 & & & & 0.557 & 208 & 0 & -9.81 & & 0.270 & & \\
\hline 24 & 3 & 0.98 & & & & 0.590 & 210 & 0 & -2.73 & & & & 0.480 \\
\hline 25 & 3 & -0.71 & & & & 0.540 & 212 & 4 & -0.03 & & & & 0.560 \\
\hline 26 & 0 & 3.68 & & 0.670 & & & 215 & 1 & 1.99 & & & & 0.620 \\
\hline 28 & 0 & -2.06 & & 0.500 & & & 224 & 0 & 2.83 & & 0.645 & & \\
\hline 32 & 4 & 0.47 & & & & 0.575 & 234 & 2 & -1.05 & & 0.530 & & \\
\hline 36 & 2 & 1.32 & & & & 0.600 & 236 & 0 & 4.35 & & 0.690 & & \\
\hline 39 & 4 & 0.30 & & & & 0.570 & 241 & 3 & 0.64 & & & & 0.580 \\
\hline 40 & 4 & -0.37 & & & & 0.550 & & & & & & & \\
\hline 42 & 4 & -0.03 & & & & 0.560 & & & & & & & \\
\hline 46 & 4 & 0.34 & & & & 0.571 & & & & & & & \\
\hline 50 & 4 & -0.37 & & & & 0.550 & & & & & & & \\
\hline 52 & 2 & -1.45 & 0.518 & & & & & & & & & & \\
\hline 54 & 4 & 0.24 & & & & 0.568 & & & & & & & \\
\hline 55 & 0 & -2.06 & & & & 0.500 & & & & & & & \\
\hline 57 & 3 & -0.71 & & & & 0.540 & & & & & & & \\
\hline 58 & 0 & -4.42 & & & & 0.430 & & & & & & & \\
\hline 59 & 4 & -0.37 & & & & 0.550 & & & & & & & \\
\hline 69 & 4 & -0.03 & & & & 0.560 & & & & & & & \\
\hline 70 & 4 & -0.03 & & & & 0.560 & & & & & & & \\
\hline 76 & 4 & 0.00 & & 0.561 & & & & & & & & & \\
\hline 80 & 0 & 4.01 & & & & 0.680 & & & & & & & \\
\hline 81 & 4 & -0.03 & & & & 0.560 & & & & & & & \\
\hline 83 & 1 & 1.65 & & & & 0.610 & & & & & & & \\
\hline 85 & 4 & -0.03 & & & & 0.560 & & & & & & & \\
\hline 86 & 0 & -3.74 & & 0.450 & & & & & & & & & \\
\hline 89 & 4 & -0.13 & & & & 0.557 & & & & & & & \\
\hline 93 & 0 & 14.13 & & & & 0.980 & & & & & & & \\
\hline 94 & 4 & -0.37 & & & & 0.550 & & & & & & & \\
\hline 96 & 3 & 0.64 & & & & 0.580 & & & & & & & \\
\hline 97 & 3 & 0.74 & & & & 0.583 & & & & & & & \\
\hline 100 & 2 & -1.05 & & & & 0.530 & & & & & & & \\
\hline 102 & 3 & 0.64 & & 0.580 & & & & & & & & & \\
\hline 105 & 3 & -0.71 & & 0.540 & & & & & & & & & \\
\hline 107 & 3 & 0.98 & & & & 0.590 & & & & & & & \\
\hline 109 & 3 & 0.64 & & & & 0.580 & & & & & & & \\
\hline 114 & 3 & 0.98 & & & & 0.590 & & & & & & & \\
\hline 119 & 3 & 0.98 & & & & 0.590 & & & & & & & \\
\hline 127 & 4 & -0.20 & & 0.555 & & & & & & & & & \\
\hline 128 & 4 & -0.03 & & 0.560 & & & & & & & & & \\
\hline 129 & 2 & -1.45 & & 0.518 & & & & & & & & & \\
\hline 134 & 3 & -0.74 & & & & 0.539 & & & & & & & \\
\hline 138 & 1 & 1.65 & & 0.610 & & & & & & & & & \\
\hline 140 & 2 & 1.32 & & & & 0.600 & & & & & & & \\
\hline 141 & 1 & -1.72 & & & & 0.510 & & & & & & & \\
\hline
\end{tabular}


13. Statistical summary of reported data for standard reference water sample M-134 (major constituents)--Continued K (Potassium)

$\mathrm{mg} / \mathrm{l}$

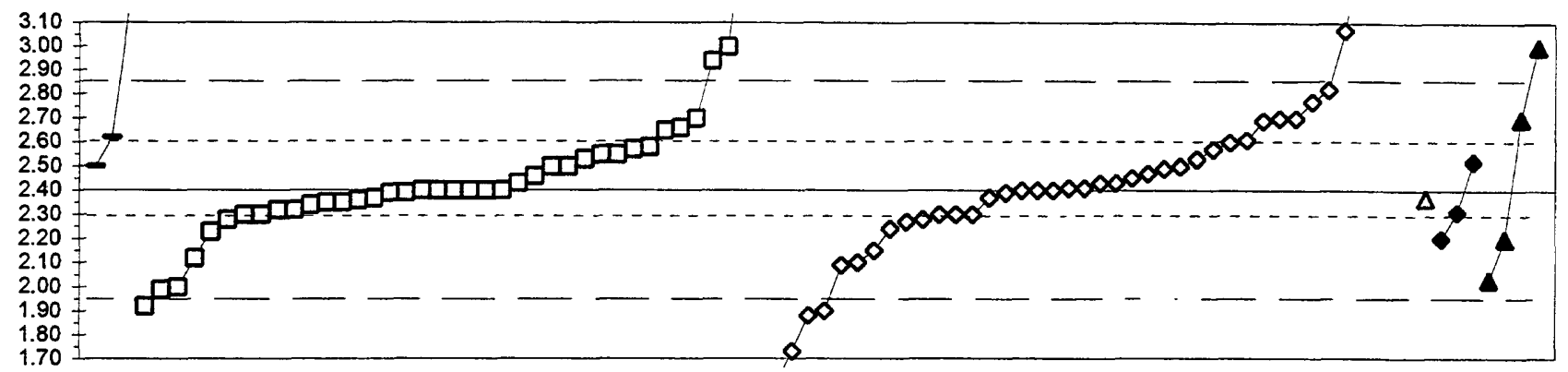

\begin{tabular}{|c|c|c|c|c|c|c|c|c|c|c|c|c|c|c|c|c|c|}
\hline \multirow[b]{2}{*}{ Lab } & \multicolumn{2}{|c|}{$\begin{array}{r}\text { 0. Other } \\
\text { 1. AA: direct air } \\
\text { 4. ICP } \\
\mathrm{N}= \\
\text { Minimum }= \\
\text { Maximum }= \\
\text { Median }= \\
\text { F-pseudosigma }=\end{array}$} & $\begin{array}{r}3 \\
2.50 \\
3.15 \\
2.62\end{array}$ & $\begin{array}{r}39 \\
1.92 \\
4.00 \\
2.40 \\
0.16 \\
\end{array}$ & $\begin{array}{l}\text { 5. DCP } \\
\text { 6. ICP/M } \\
\text { 7. Ion ch } \\
40 \\
1.60 \\
5.00 \\
2.42 \\
0.27 \\
\end{array}$ & $\begin{array}{c} \\
\text { matog } \\
1 \\
2.37\end{array}$ & $\begin{array}{r}\text { phy } \\
3 \\
2.20 \\
2.52 \\
2.31\end{array}$ & $\begin{array}{r}4 \\
2.03 \\
3.00 \\
2.45\end{array}$ & \multirow[t]{2}{*}{ Lab } & \multirow{2}{*}{\multicolumn{2}{|c|}{$\begin{aligned} M P V & = \\
\text { E-pseudosigma } & = \\
N & = \\
H U & = \\
H I & = \\
\text { Rating Z-value } & \end{aligned}$}} & \multirow[t]{2}{*}{$\begin{array}{r}2.40 \\
0.22 \\
90 \\
2.60 \\
2.30 \\
\\
\\
\\
0\end{array}$} & \multirow[b]{2}{*}{1} & \multirow[b]{2}{*}{4} & \multirow[b]{2}{*}{5} & \multirow[b]{2}{*}{6} & \multirow[b]{2}{*}{7} \\
\hline & Rating & Z-value & 0 & 1 & 4 & 5 & 6 & 7 & & & & & & & & & \\
\hline 1 & 4 & -0.04 & & 2.39 & & & & & 107 & 4 & 0.13 & & 2.43 & & & & \\
\hline 3 & 3 & 0.76 & & 2.57 & & & & & 109 & 2 & 1.12 & & 2.65 & & & & \\
\hline 7 & 1 & 1.66 & & & 2.77 & & & & 111 & 0 & 2.43 & & 2.94 & . & & & \\
\hline 9 & 2 & 1.30 & & & 2.69 & & & & 114 & 4 & 0.45 & & 2.50 & & & & \\
\hline 10 & 4 & -0.22 & & 2.35 & & & & & 116 & 0 & 11.69 & & & 5.00 & & & \\
\hline 11 & 4 & 0.40 & & & 2.49 & & & & 119 & 0 & -2.25 & & & 1.90 & & & \\
\hline 13 & 2 & -1.35 & & & 2.10 & & & & 121 & 4 & 0.00 & & 2.40 & & & & \\
\hline 15 & 4 & -0.04 & & & 2.39 & & & & 127 & 3 & 0.67 & & 2.55 & & & & \\
\hline 16 & 1 & -1.80 & & 2.00 & & & & & 128 & 0 & -3.01 & & & 1.73 & & & \\
\hline 18 & 4 & -0.45 & & & 2.30 & & & & 129 & 0 & 7.19 & & 4.00 & & & & \\
\hline 19 & 4 & 0.45 & & & 2.50 & & & & 132 & 4 & 0.23 & & & 2.45 & & & \\
\hline 24 & 4 & -0.45 & & & 2.30 & & & & 134 & 4 & -0.14 & & 2.37 & & & & \\
\hline 25 & 2 & -1.39 & & & 2.09 & & & & 136 & 3 & -0.76 & & 2.23 & & & & \\
\hline 26 & 4 & 0.31 & & & 2.47 & & & & 138 & 4 & -0.13 & & & 2.37 & & & \\
\hline 27 & 3 & 0.81 & & 2.58 & & & & & 140 & 2 & -1.26 & & 2.12 & & & & \\
\hline 28 & 0 & 9.89 & & & 4.60 & & & & 141 & 4 & 0.04 & & & 2.41 & & & \\
\hline 32 & 3 & 0.54 & & & & & 2.52 & & 142 & 1 & 1.89 & & & 2.82 & & & \\
\hline 33 & 4 & -0.13 & & & & 2.37 & & & 145 & 3 & -0.58 & & & 2.27 & & & \\
\hline 36 & 1 & -1.84 & & 1.99 & & & & & 146 & 0 & 3.37 & 3.15 & & & & & \\
\hline 38 & 4 & -0.04 & & 2.39 & & & & & 151 & 3 & -0.54 & & 2.28 & & & & \\
\hline 40 & 4 & 0.04 & & & 2.41 & & & & 153 & 3 & -0.90 & & & & & & 2.20 \\
\hline 42 & 4 & 0.00 & & & 2.40 & & & & 154 & 0 & 4.50 & & & 3.40 & & & \\
\hline 43 & 4 & 0.00 & & & 2.40 & & & & 180 & 0 & 3.01 & & & 3.07 & & & \\
\hline 48 & 2 & -1.12 & & & 2.15 & & & & 182 & 3 & 0.59 & & & 2.53 & & & \\
\hline 50 & 4 & 0.00 & & 2.40 & & & & & 190 & 1 & -1.66 & & & & & & 2.03 \\
\hline 52 & 4 & 0.00 & & & 2.40 & & & & 191 & 4 & -0.40 & & & & & 2.31 & \\
\hline 54 & 4 & 0.00 & & 2.40 & & & & & 194 & 3 & 0.58 & & 2.53 & & & & \\
\hline 55 & 4 & 0.27 & & 2.46 & & & & & 196 & 4 & -0.45 & & 2.30 & & & & \\
\hline 56 & 4 & 0.45 & 2.50 & & & & & & 210 & 0 & 8.09 & & & 4.20 & & & \\
\hline 57 & 0 & 2.70 & & 3.00 & & & & & 212 & 3 & 0.90 & & & 2.60 & & & \\
\hline 58 & 4 & 0.45 & & 2.50 & & & & & 215 & 0 & -2.34 & & & 1.88 & & & \\
\hline 59 & 2 & 1.35 & & & 2.70 & & & & 219 & 3 & -0.90 & & & & & 2.20 & \\
\hline 64 & 4 & -0.22 & & 2.35 & & & & & 221 & 3 & 0.67 & & 2.55 & & & & \\
\hline 68 & 0 & & & & $<0.15$ & & & & 224 & 4 & 0.13 & & & 2.43 & & & \\
\hline 69 & 3 & 0.99 & 2.62 & & & & & & 226 & 4 & 0.00 & & 2.40 & & & & \\
\hline 70 & 3 & -0.54 & & & 2.28 & & & & 230 & 2 & 1.35 & & & & & & 2.70 \\
\hline 75 & 4 & 0.00 & & 2.40 & & & & & 231 & 4 & -0.27 & & 2.34 & & & & \\
\hline 80 & 2 & 1.35 & & 2.70 & & & & & 234 & 3 & 0.94 & & & 2.61 & & & \\
\hline 81 & 4 & -0.45 & & & 2.30 & & & & 236 & 3 & -0.72 & & & 2.24 & & & \\
\hline 83 & 0 & -2.16 & & 1.92 & & & & & 237 & 0 & 2.70 & & & & & & 3.00 \\
\hline 85 & 2 & 1.17 & & 2.66 & & & & & 241 & 4 & -0.36 & & 2.32 & & & & \\
\hline 86 & 4 & 0.13 & & & 2.43 & & & & & & & & & & & & \\
\hline 87 & 4 & -0.36 & & 2.32 & & & & & & & & & & & & & \\
\hline 89 & 4 & -0.45 & & 2.30 & & & & & & & & & & & & & \\
\hline 92 & 0 & 4.95 & & 3.50 & & & & & & & & & & & & & \\
\hline 97 & 4 & -0.18 & & 2.36 & & & & & & & & & & & & & \\
\hline 101 & 4 & 0.00 & & 2.40 & & & & & & & & & & & & & \\
\hline 102 & 0 & -3.60 & & & 1.60 & & & & & & & & & & & & \\
\hline 103 & 2 & 1.35 & & & 2.70 & & & & & & & & & & & & \\
\hline 105 & 3 & 0.76 & & & 2.57 & & & & & & & & & & & & \\
\hline 107 & 4 & 0.13 & & 2.43 & & & & & & & & & & & & & \\
\hline 109 & 2 & 1.12 & & 2.65 & & & & & & & & & & & & & \\
\hline 111 & 0 & 2.43 & & 2.94 & & & & & & & & & & & & & \\
\hline 114 & 4 & 0.45 & & 2.50 & & & & & & & & & & & & & \\
\hline 116 & 0 & 11.69 & & & 5.00 & & & & & & & & & & & & \\
\hline
\end{tabular}


Table 13. Statistical summary of reported data for standard reference water sample M-134 (major constituents)--Continued Mg (Magnesium) $\mathrm{mg} / \mathrm{l}$

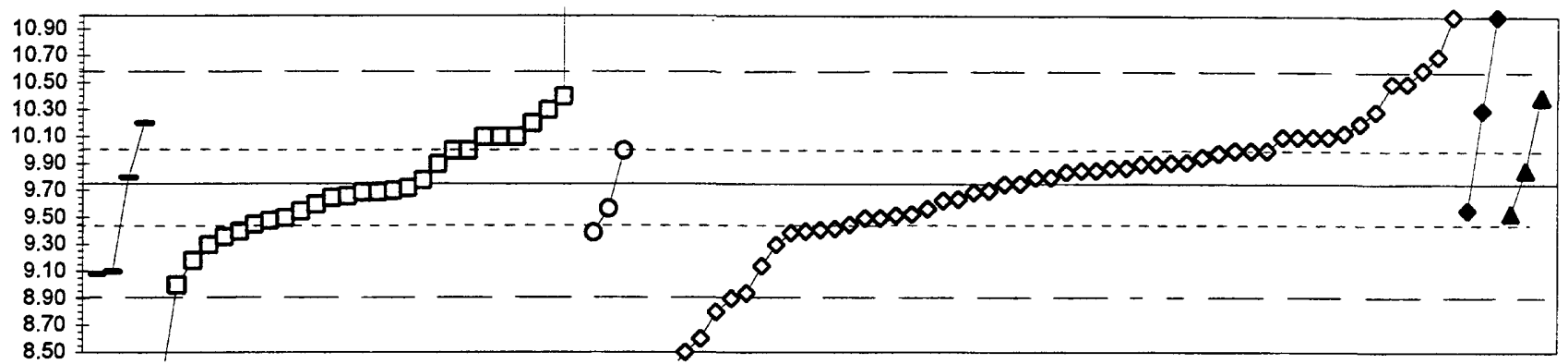

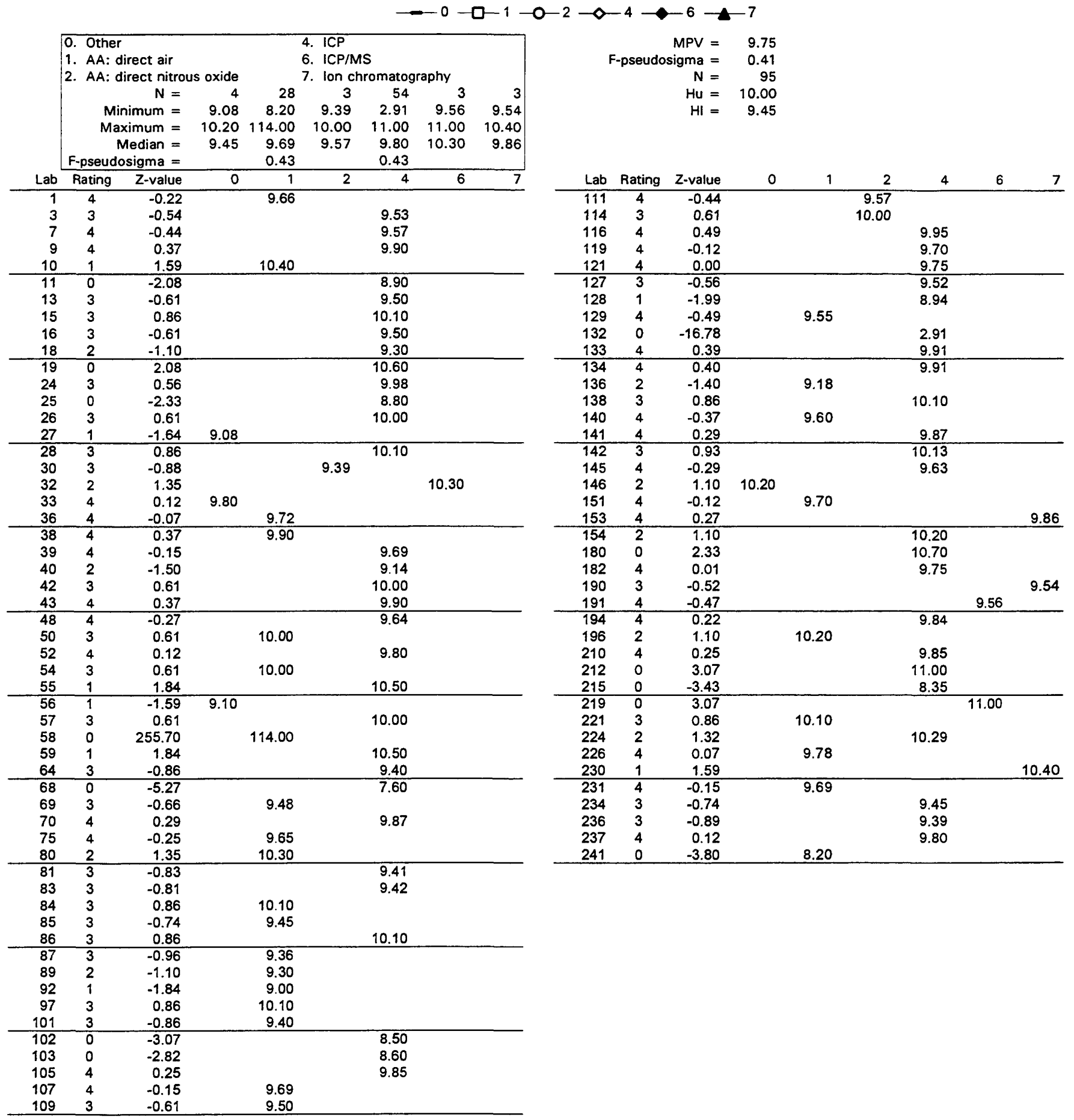


Table 13. Statistical summary of reported data for standard reference water sample M-134 (major constituents)--Continued $\mathrm{Na}$ (Sodium) $\mathrm{mg} / \mathrm{l}$

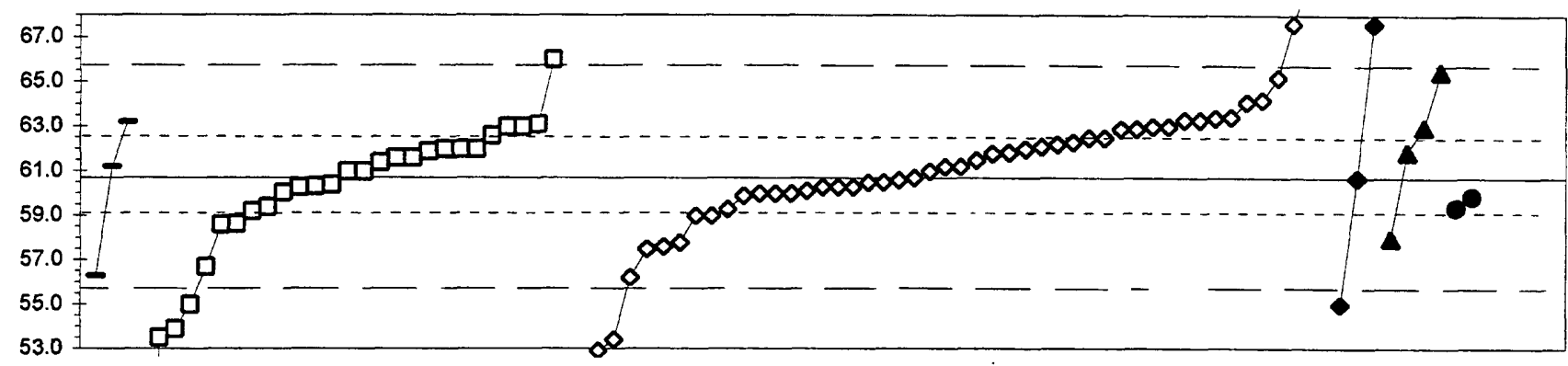

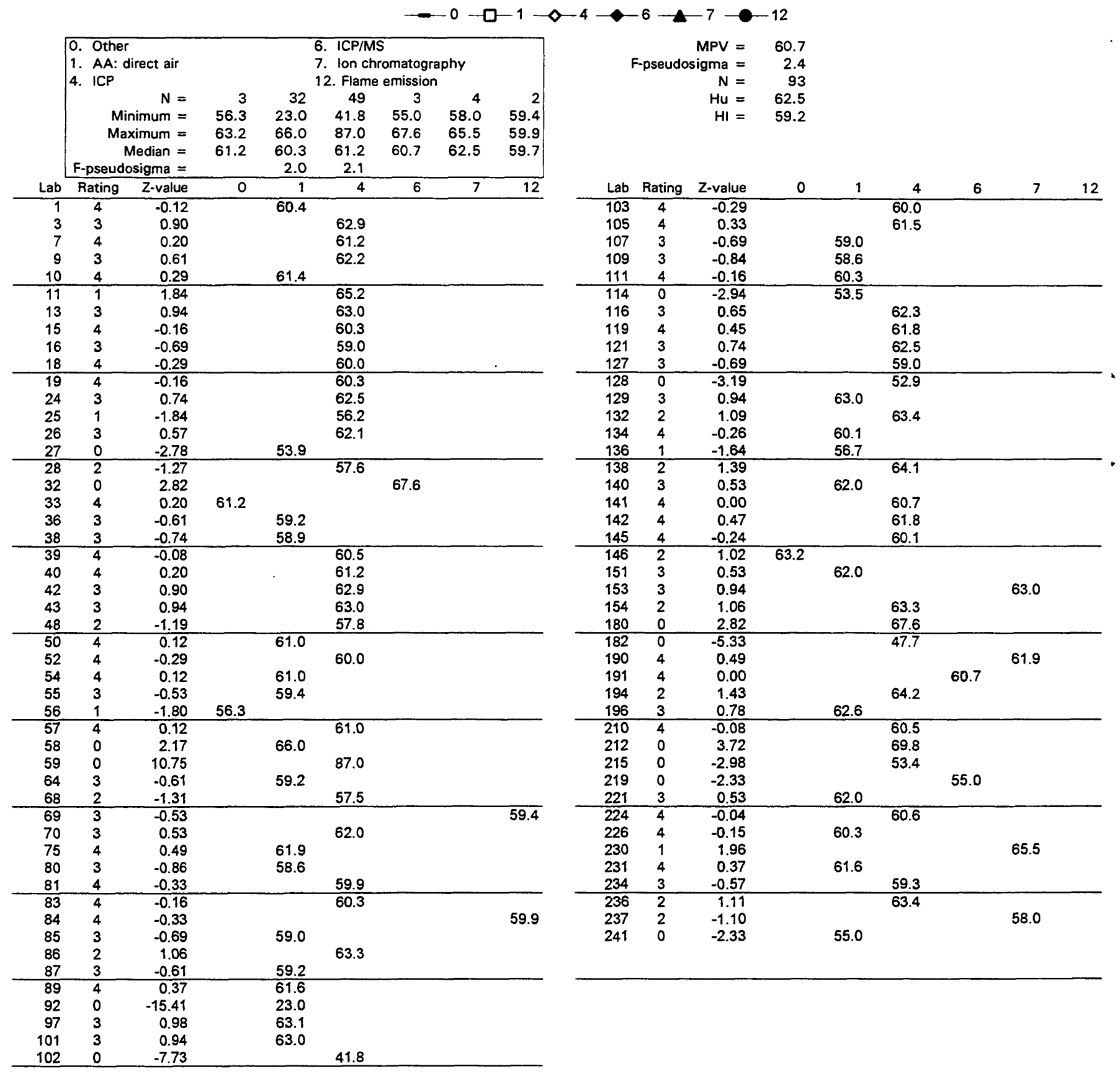


Table 13. Statistical summary of reported data for standard reference water sample M-134 (major constituents)--Continued total P Itotal Phosphorus $\mathbf{m g} / \mathbf{l}$

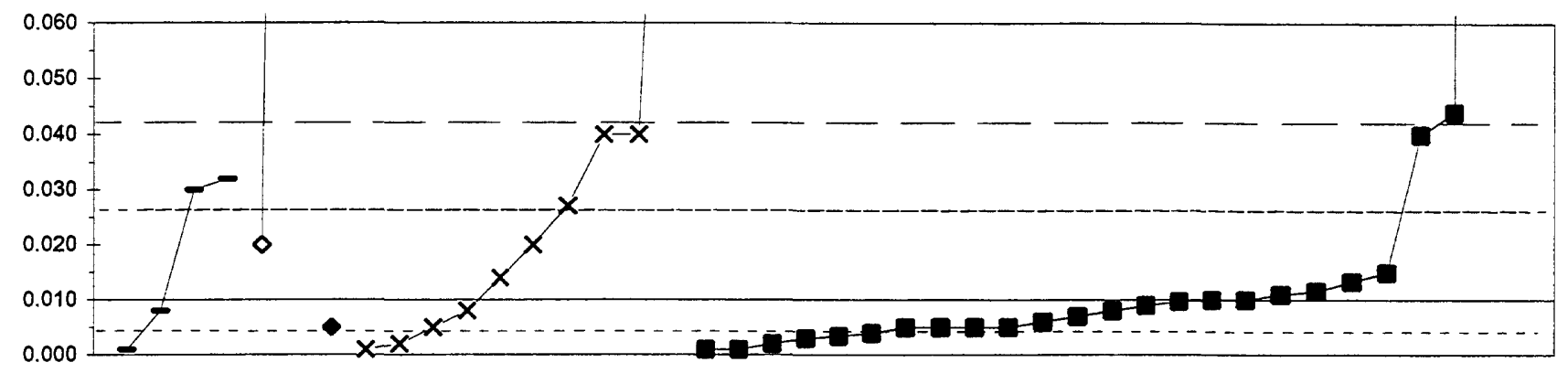

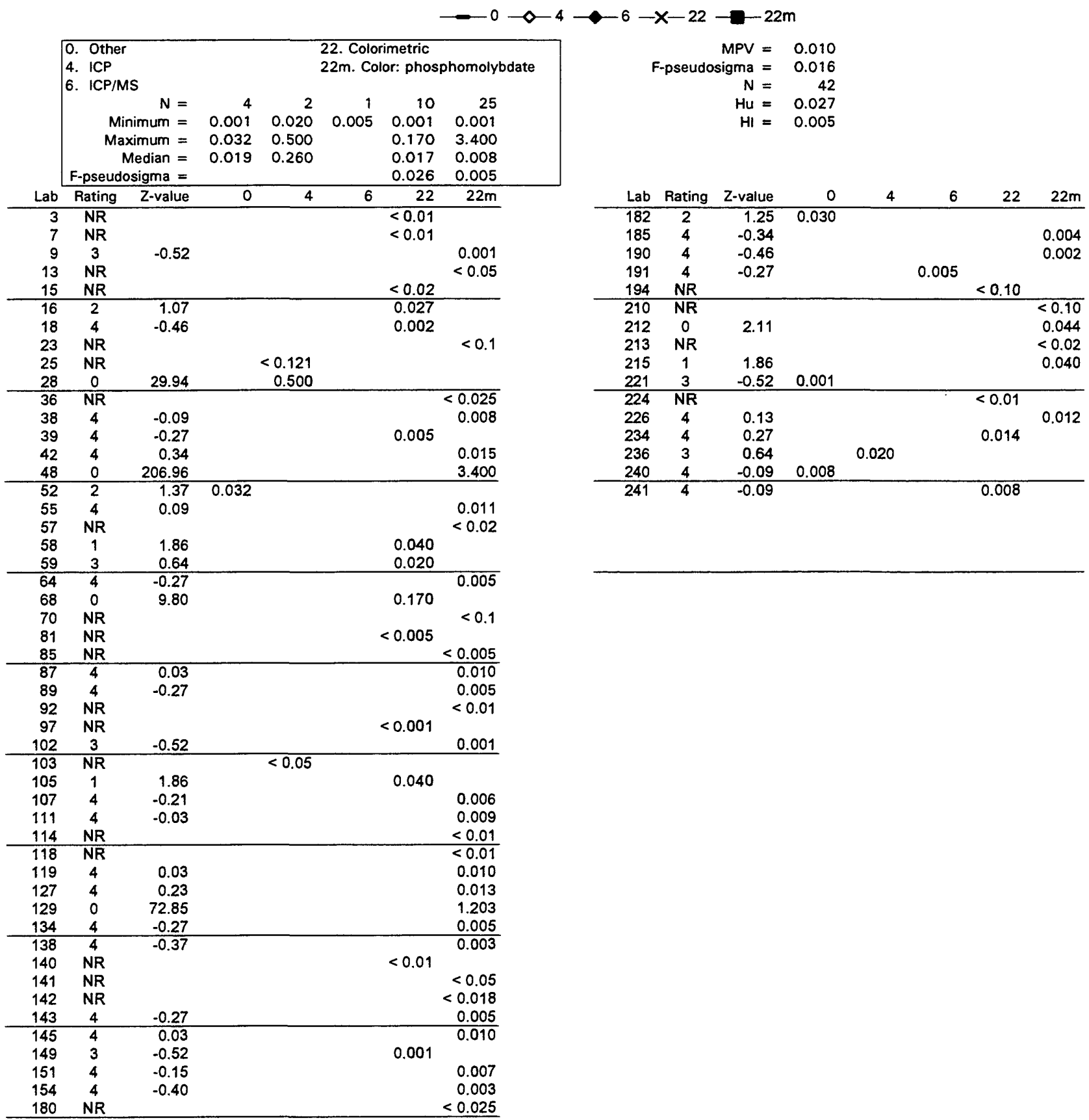


Table 13. Statistical summary of reported data for standard reference water sample M-134 (major constituents)--Continued pH

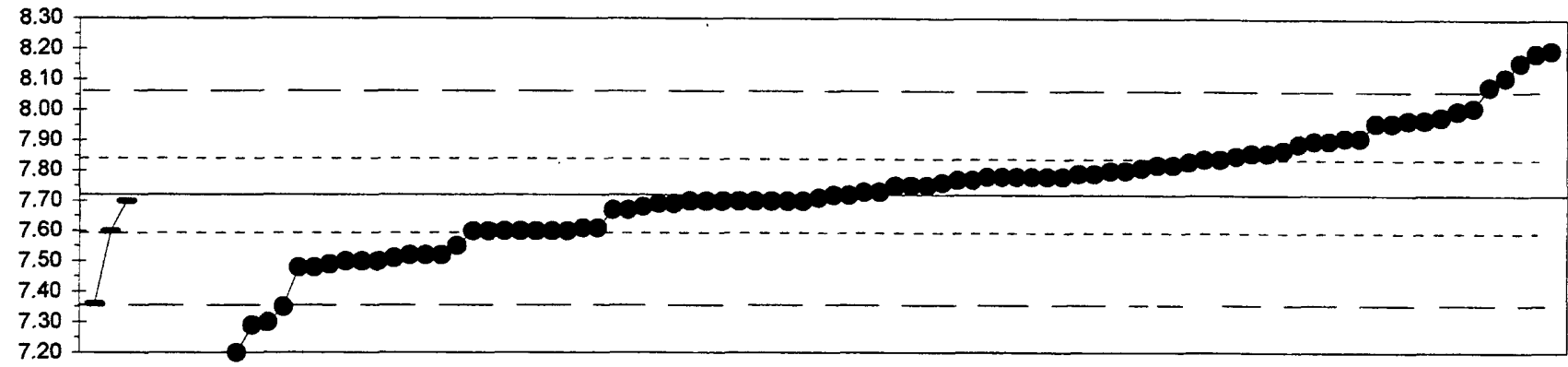

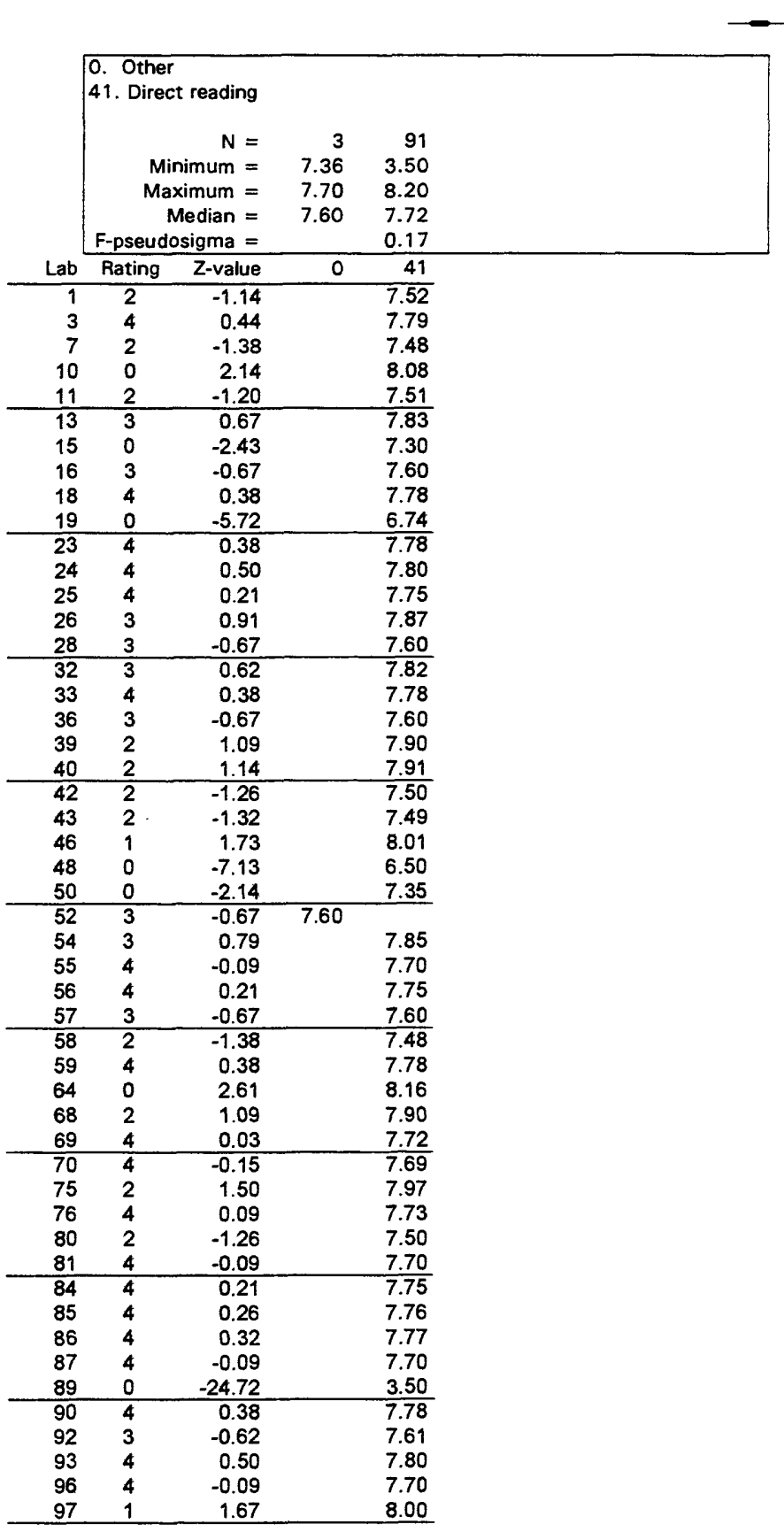

$0-41$

\begin{tabular}{|c|c|c|c|c|}
\hline Lab & Rating & $Z$-value & 0 & 41 \\
\hline 100 & 3 & -0.62 & & 7.61 \\
\hline 101 & 4 & -0.21 & & 7.68 \\
\hline 105 & 4 & 0.32 & & 7.77 \\
\hline 107 & 0 & -17.33 & & 4.76 \\
\hline 109 & 0 & -5.48 & & 6.78 \\
\hline 111 & 3 & 0.73 & & 7.84 \\
\hline 114 & 3 & -0.67 & & 7.60 \\
\hline 118 & 2 & -1.26 & & 7.50 \\
\hline 11.9 & 2 & 1.44 & & 7.96 \\
\hline 127 & 4 & -0.09 & & 7.70 \\
\hline 128 & 4 & -0.15 & & 7.69 \\
\hline 129 & 3 & 0.56 & & 7.81 \\
\hline 132 & 4 & 0.09 & & 7.73 \\
\hline 134 & 4 & -0.03 & & 7.71 \\
\hline 136 & 3 & 0.73 & & 7.84 \\
\hline 138 & 1 & 1.55 & & 7.98 \\
\hline 140 & 2 & -1.14 & & 7.52 \\
\hline 141 & 3 & 0.85 & & 7.86 \\
\hline 142 & 4 & 0.03 & & 7.72 \\
\hline 143 & 3 & 0.62 & & 7.82 \\
\hline 145 & 0 & -3.02 & & 7.20 \\
\hline 146 & 0 & -2.08 & 7.36 & \\
\hline 149 & 2 & 1.44 & & 7.96 \\
\hline 151 & 4 & -0.26 & & 7.67 \\
\hline 153 & 2 & -1.14 & & 7.52 \\
\hline 154 & 0 & 2.79 & & 8.19 \\
\hline 180 & 4 & -0.09 & & 7.70 \\
\hline 182 & 3 & -0.67 & & 7.60 \\
\hline 190 & 2 & 1.14 & & 7.91 \\
\hline 194 & 3 & -0.97 & & 7.55 \\
\hline 196 & 0 & -7.48 & & 6.44 \\
\hline 203 & 4 & -0.26 & & 7.67 \\
\hline 204 & 0 & -2.49 & & 7.29 \\
\hline 210 & 0 & 2.32 & & 8.11 \\
\hline 212 & 3 & -0.67 & & 7.60 \\
\hline 213 & 4 & 0.38 & & 7.78 \\
\hline 215 & 3 & 0.85 & & 7.86 \\
\hline 221 & 4 & -0.09 & 7.70 & \\
\hline 224 & 4 & -0.09 & & 7.70 \\
\hline 234 & 2 & 1.03 & & 7.89 \\
\hline 236 & 4 & -0.09 & & 7.70 \\
\hline 237 & 0 & 2.84 & & 8.20 \\
\hline 240 & 2 & 1.50 & & 7.97 \\
\hline 241 & 4 & 0.44 & & 7.79 \\
\hline
\end{tabular}


Table 13. Statistical summary of reported data for standard reference water sample M-134 (major constituents)--Continued SiO2 (Silica) $\mathrm{mg} / \mathrm{l}$

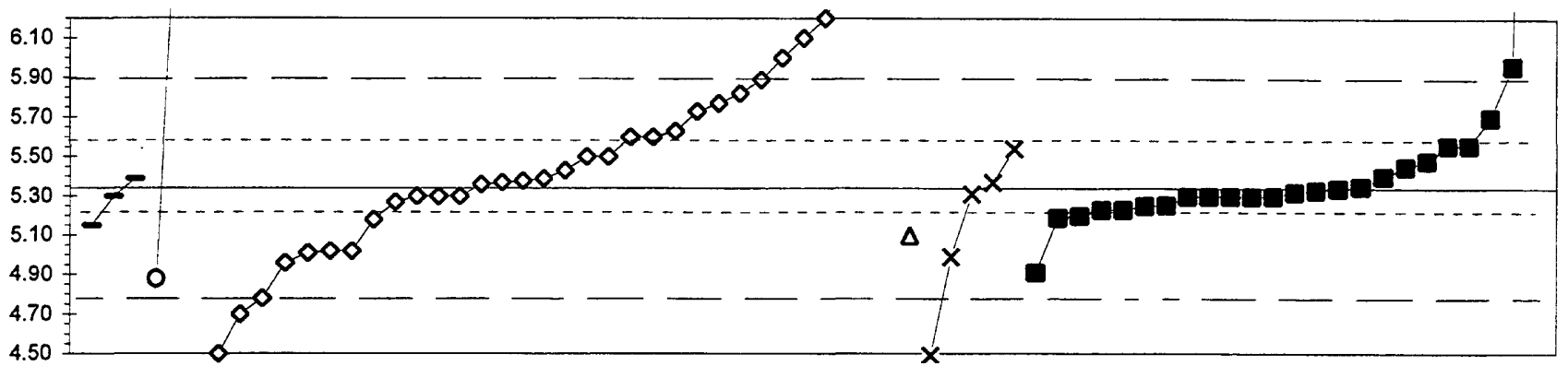

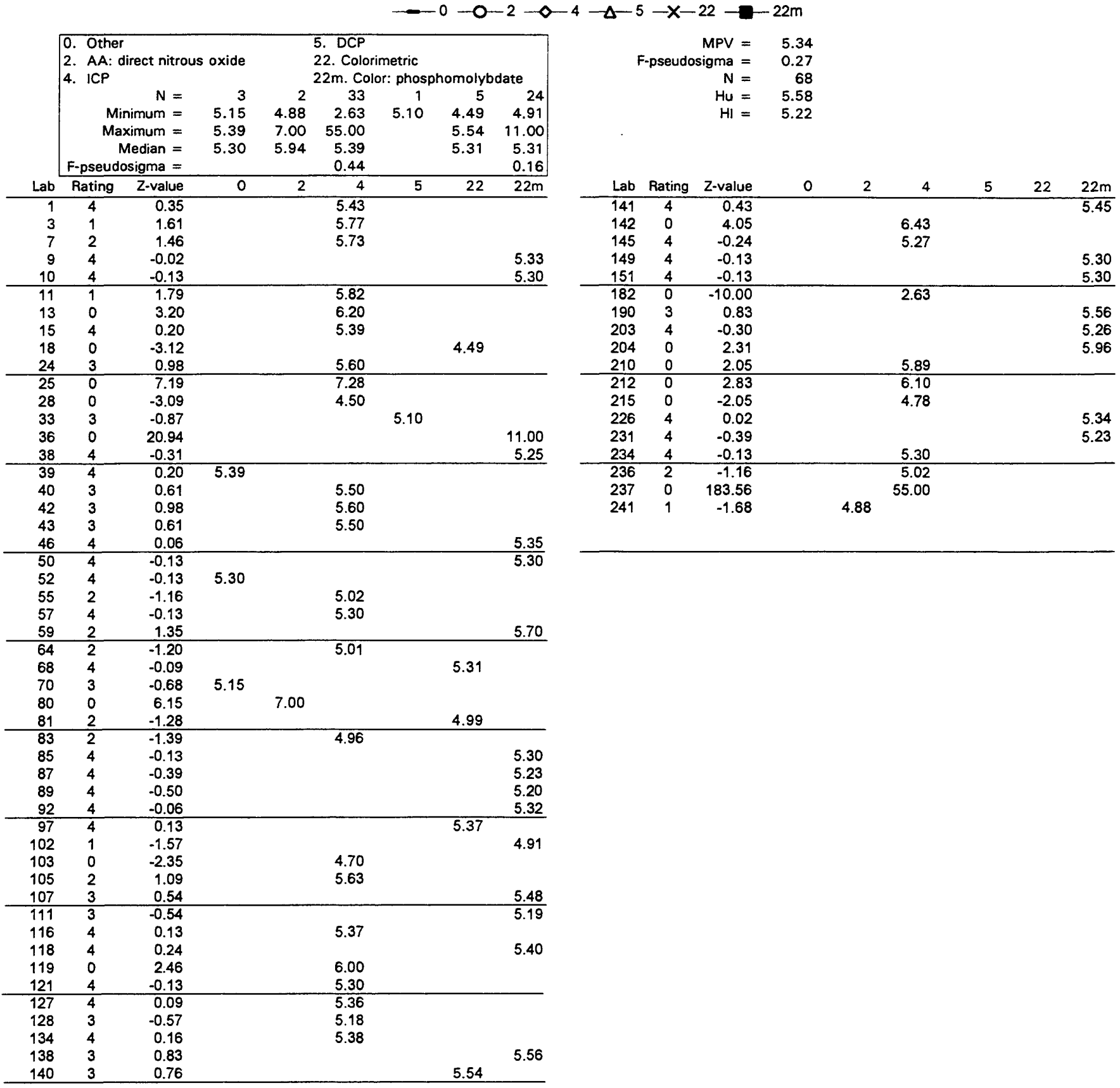


Table 13. Statistical summary of reported data for standard reference water sample $M-134$ (major constituents)--Continued SO4 (Sulfate) $\mathrm{mg} / \mathrm{l}$

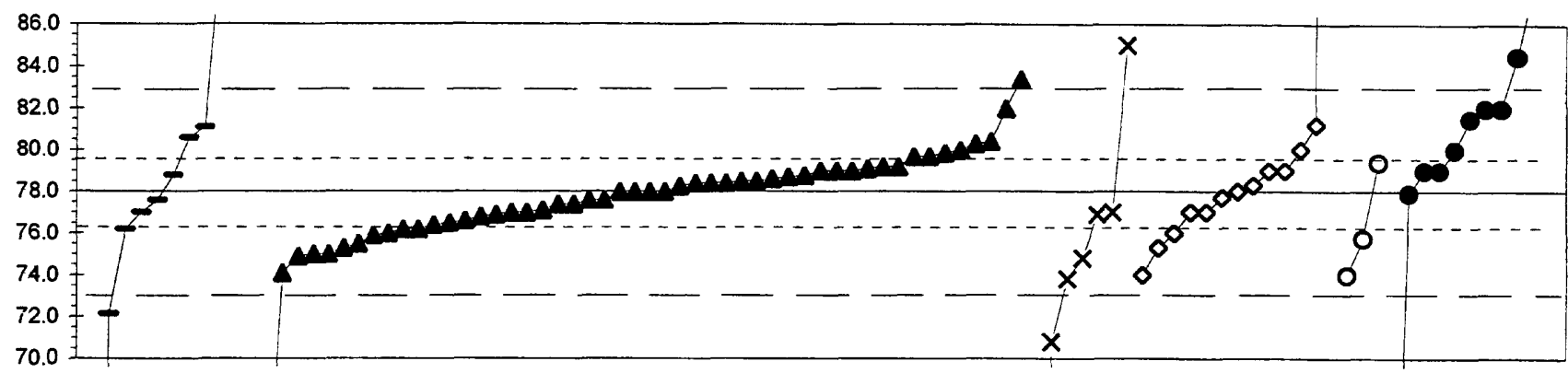

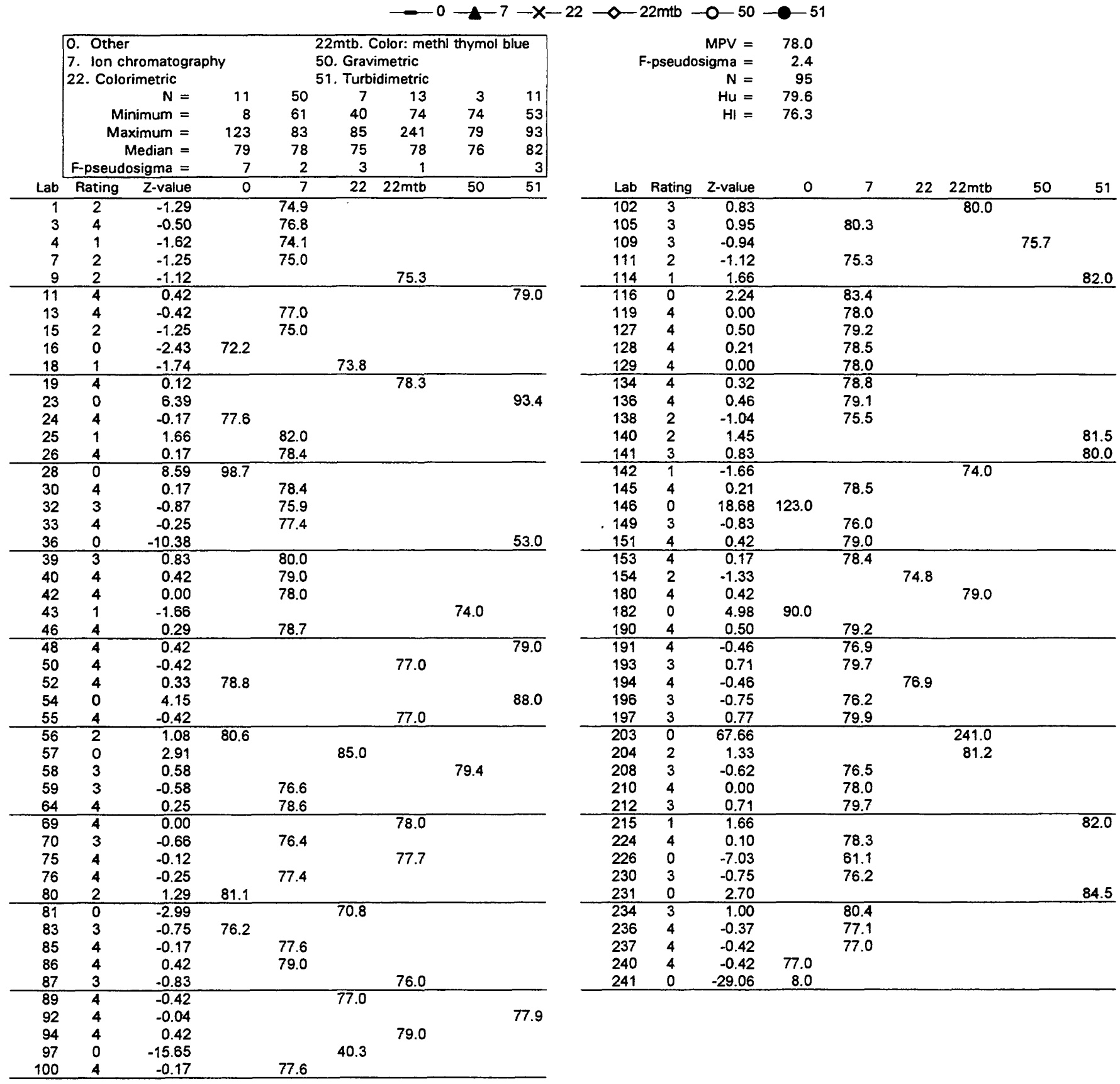


Table 13. Statistical summary of reported data for standard reference water sample M-134 (major constituents)--Continued Sp Cond (Specific Conductance) $\mu \mathrm{S} / \mathrm{cm}$

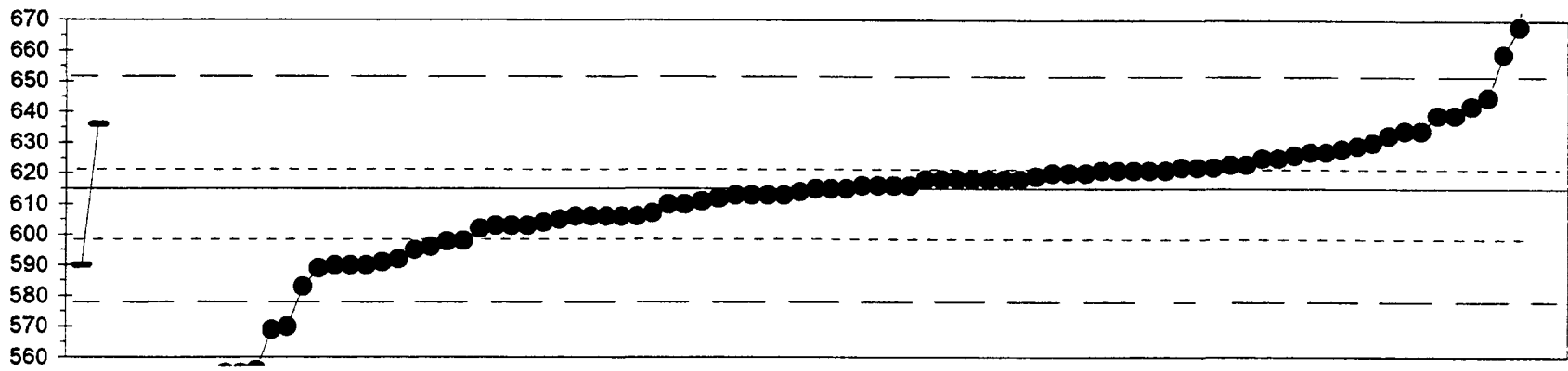

$-0$

$-41$

\begin{tabular}{|c|c|c|c|c|}
\hline \multirow[b]{2}{*}{ Lab } & Fa & $\begin{aligned} N & = \\
\text { imum } & = \\
\text { imum } & = \\
\text { ledian } & = \\
\text { sigma } & =\end{aligned}$ & $\begin{array}{r}2 \\
590 \\
636 \\
613\end{array}$ & $\begin{array}{r}91 \\
58 \\
746 \\
615 \\
16\end{array}$ \\
\hline & Rating & Z-value & 0 & 41 \\
\hline 1 & 3 & -0.51 & & $\overline{606}$ \\
\hline 3 & 4 & 0.28 & & 620 \\
\hline 7 & 3 & -0.56 & & 605 \\
\hline 9 & 0 & -2.59 & & 569 \\
\hline 10 & 4 & 0.00 & & 615 \\
\hline 11 & 3 & -0.51 & & 606 \\
\hline 13 & 4 & 0.45 & & 623 \\
\hline 15 & 1 & 1.69 & & 645 \\
\hline 16 & 3 & -0.97 & & 598 \\
\hline 18 & 3 & -0.73 & & 602 \\
\hline 19 & 2 & 1.07 & & 634 \\
\hline 23 & 2 & -1.29 & & 592 \\
\hline 24 & 4 & 0.17 & & 618 \\
\hline 25 & 3 & 0.84 & & 630 \\
\hline 26 & 3 & 0.67 & & 627 \\
\hline 27 & 4 & -0.11 & & 613 \\
\hline 28 & 0 & 2.98 & & 668 \\
\hline 32 & 4 & -0.22 & & 611 \\
\hline 33 & 4 & 0.06 & & 616 \\
\hline 36 & 1 & 1.52 & & 642 \\
\hline 38 & 3 & 0.98 & & 633 \\
\hline 39 & 0 & -7.53 & & 481 \\
\hline 40 & 4 & 0.17 & & 618 \\
\hline 42 & 3 & 0.79 & & 629 \\
\hline 43 & 4 & 0.06 & & 616 \\
\hline 46 & 0 & 7.36 & & 746 \\
\hline 48 & 4 & 0.17 & & 618 \\
\hline 50 & 4 & 0.00 & & 615 \\
\hline 52 & 2 & -1.41 & 590 & \\
\hline 54 & 4 & 0.34 & & 621 \\
\hline 55 & 4 & 0.28 & & 620 \\
\hline 56 & 4 & 0.06 & & 616 \\
\hline 57 & 2 & -1.41 & & 590 \\
\hline 58 & 3 & -0.67 & & 603 \\
\hline 59 & 2 & 1.07 & & 634 \\
\hline 64 & 4 & 0.17 & & 618 \\
\hline 68 & 4 & 0.34 & & 621 \\
\hline 70 & 3 & -0.67 & & 603 \\
\hline 75 & 4 & -0.11 & & 613 \\
\hline 76 & 3 & -0.51 & & 606 \\
\hline 80 & 4 & 0.17 & & 618 \\
\hline 81 & 4 & 0.39 & & 622 \\
\hline 84 & 4 & 0.34 & & 621 \\
\hline 85 & 4 & 0.22 & & 619 \\
\hline 86 & 4 & 0.17 & & 618 \\
\hline 87 & 0 & -3.37 & & 555 \\
\hline 89 & 3 & -0.96 & & 598 \\
\hline 90 & 3 & 0.73 & & 628 \\
\hline 93 & 0 & -4.75 & & 531 \\
\hline 96 & 0 & -29.94 & & 82 \\
\hline
\end{tabular}

\begin{tabular}{rcrrr} 
Lab & Rating & Z-value & 0 & 41 \\
\hline 97 & 4 & 0.39 & & 622 \\
100 & 2 & -1.12 & & 595 \\
101 & 4 & -0.11 & & 613 \\
102 & 3 & 0.56 & & 625 \\
105 & 4 & 0.17 & 618 \\
\hline 107 & 3 & 0.56 & 625 \\
109 & 2 & -1.07 & 596
\end{tabular}

$\begin{array}{llll}111 & 4 & -0.28 & 610 \\ 114 & 1 & -1.80 & 583\end{array}$

\begin{tabular}{llll}
114 & 1 & -1.80 & 583 \\
118 & 4 & -0.17 & 612 \\
\hline 119 & 3 & -0.67 & 603
\end{tabular}

$\begin{array}{rrrr}127 & 3 & -0.62 & 604 \\ 128 & 2 & 1.35 & 639\end{array}$

$\begin{array}{llll}129 & 2 & -1.35 & 591\end{array}$

\begin{tabular}{llll}
134 & 4 & 0.06 & 616 \\
\hline 136 & 4 & 0.34 & 621
\end{tabular}

$\begin{array}{llll}136 & 4 & 0.34 & 621 \\ 140 & 3 & 0.62 & 626\end{array}$

$\begin{array}{llll}141 & 4 & 0.39 & 622 \\ 142 & 3 & 0.67 & 627\end{array}$

\begin{tabular}{llll}
143 & 4 & -0.45 & 607 \\
\hline
\end{tabular}

$\begin{array}{lllll}145 & 2 & -1.46 & & 589\end{array}$

$\begin{array}{lllll}146 & 2 & 1.18 & 636 & \\ 151 & 4 & 0.00 & & 615\end{array}$

\begin{tabular}{llll}
153 & 3 & -0.51 & 606 \\
154 & 3 & -0.51 & 606 \\
\hline
\end{tabular}

\begin{tabular}{llll}
154 & 3 & -0.51 & 606 \\
\hline 180 & 0 & -7.59 & 480
\end{tabular}

$\begin{array}{llll}182 & 0 & -3.32 & 556\end{array}$

$\begin{array}{llll}183 & 0 & -3.37 & 555\end{array}$

\begin{tabular}{rrrr}
190 & 4 & 0.45 & 623 \\
193 & 4 & -0.06 & 614 \\
\hline
\end{tabular}

$\begin{array}{rrrr}194 & 0 & -2.53 & 570 \\ 196 & 0 & 2.47 & 659\end{array}$

$\begin{array}{rrrr}196 & 0 & 2.47 & 659 \\ 203 & 4 & -0.28 & 610\end{array}$

$\begin{array}{rrrr}204 & 0 & -31.31 & 58\end{array}$

\begin{tabular}{rrrr}
210 & 2 & -1.41 & 590 \\
\hline 212 & 4 & -0.11 & 613 \\
215 & 4 & 0.34 & 621 \\
224 & 2 & -1.41 & 590 \\
234 & 4 & 0.28 & 620 \\
236 & 0 & -3.65 & 550 \\
\hline 237 & 2 & 1.35 & 639 \\
240 & 0 & -3.65 & 550 \\
241 & 0 & 6.46 & 730
\end{tabular}

241

6.46

730 
Table 13. Statistical summary of reported data for standard reference water sample M-134 (major constituents)--Continued $\mathrm{Sr}$ (Strontium) $\mu \mathrm{g} / \mathrm{l}$

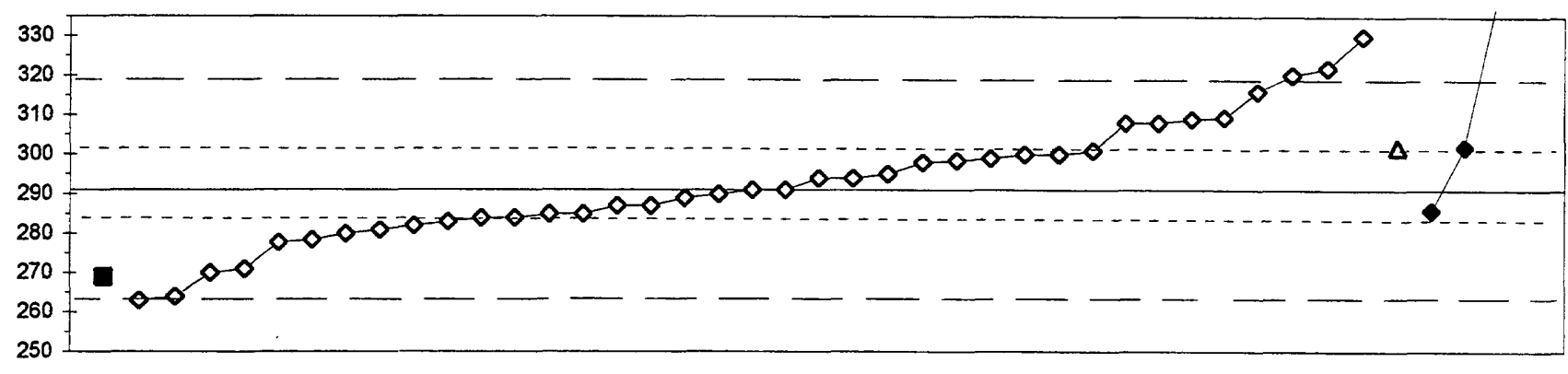

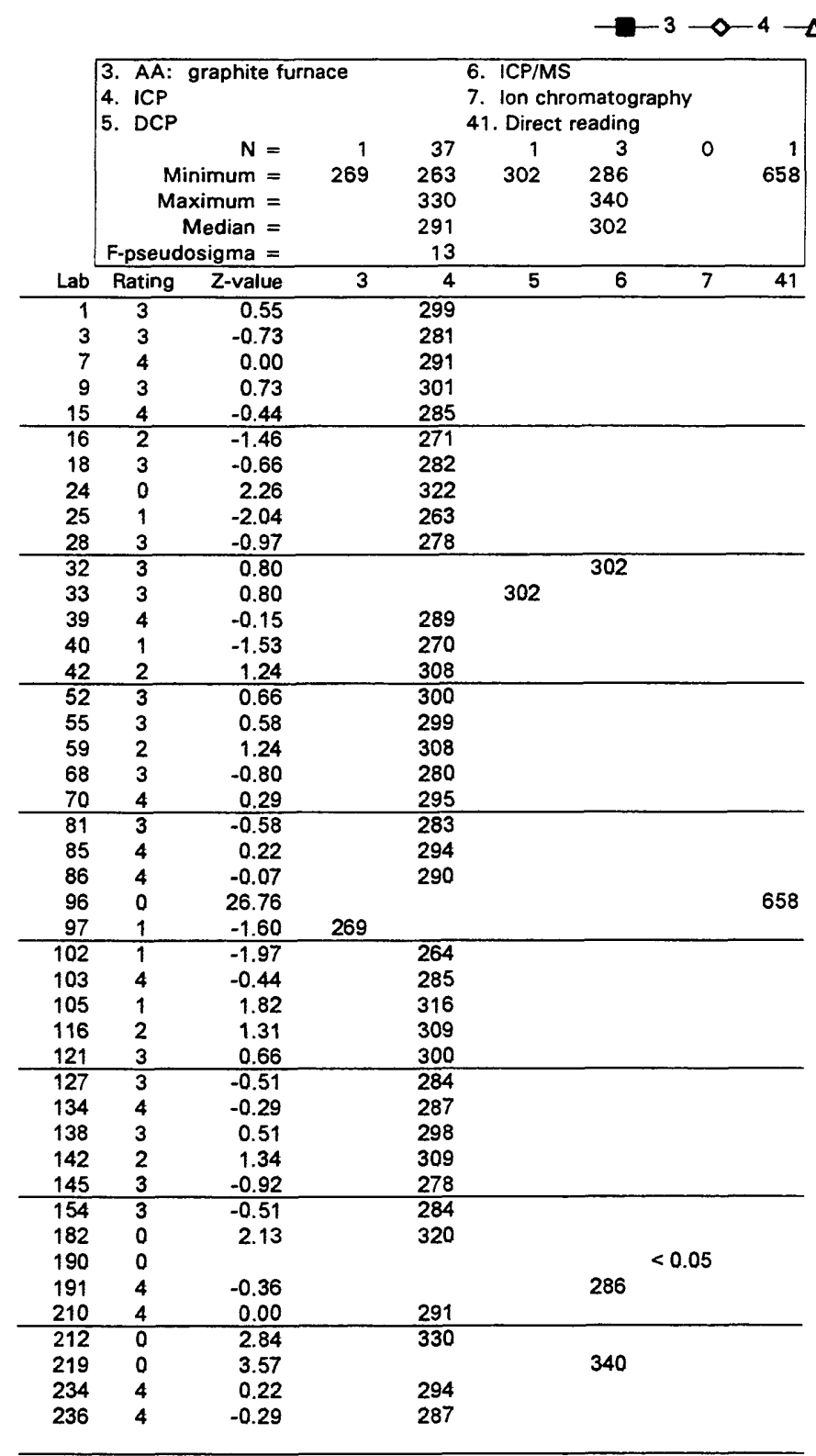


Table 13. Statistical summary of reported data for standard reference water sample M-134 (major constituents)--Continued V (Vanadium) $\mu \mathrm{g} / \mathrm{l}$

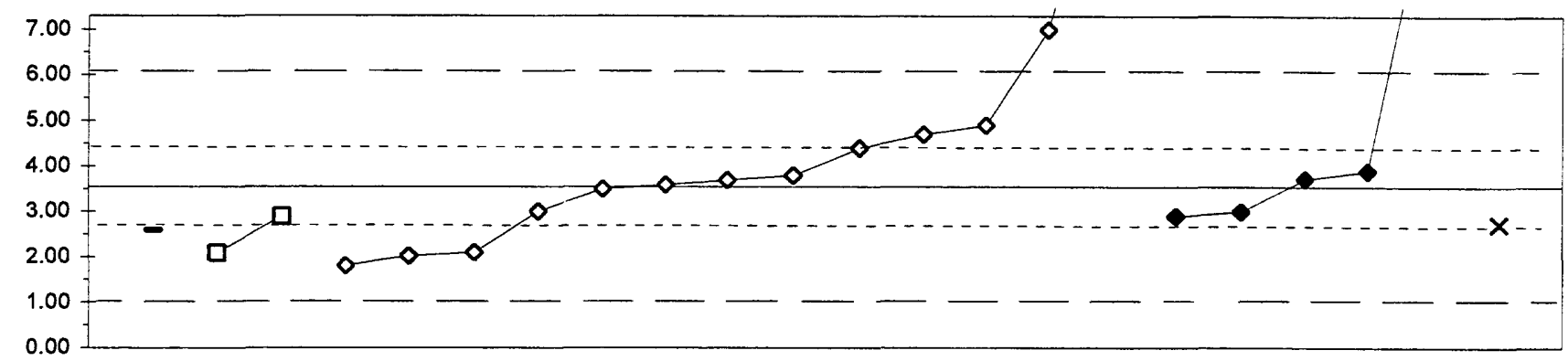

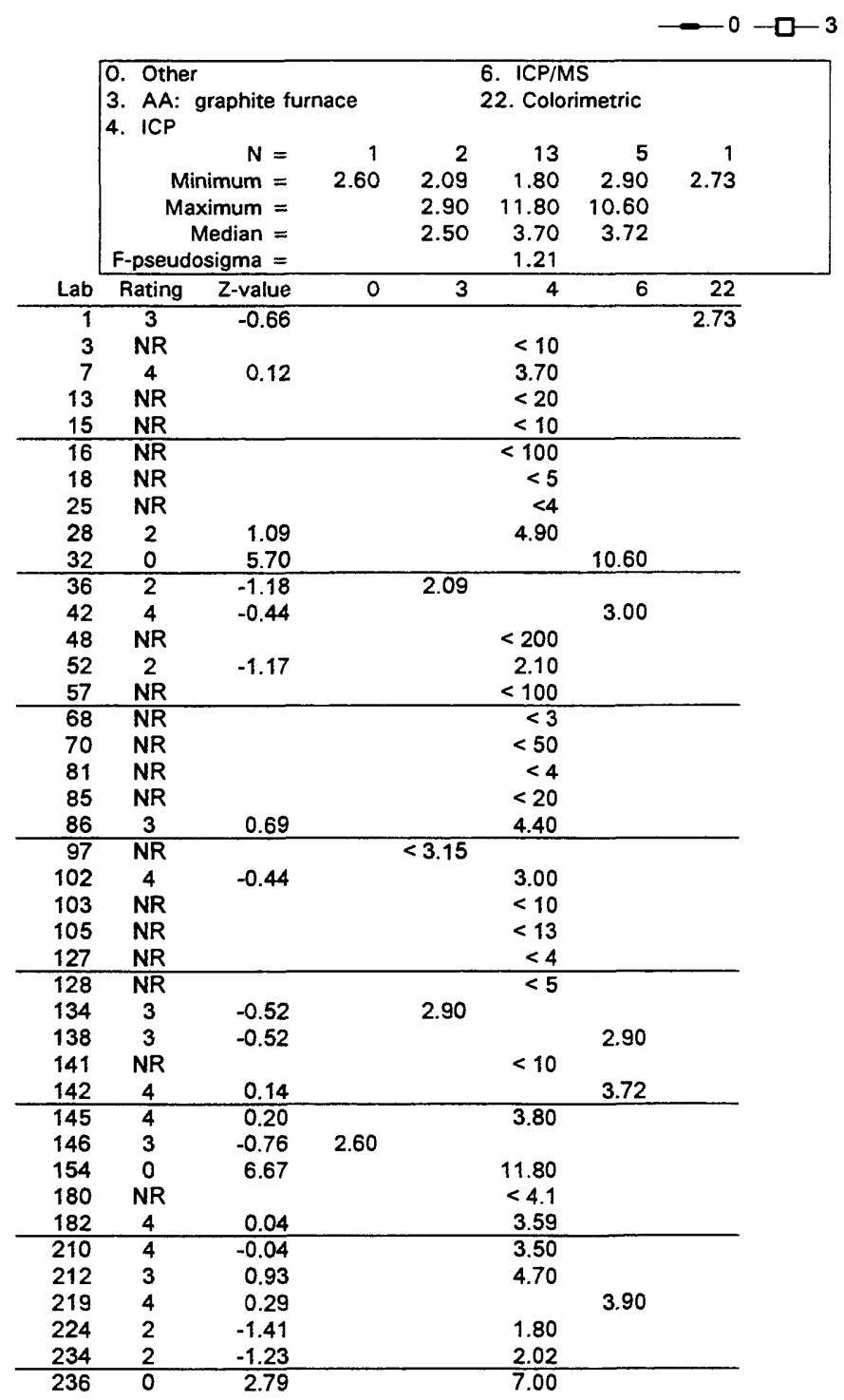


Table 14. Statistical summary of reported data for standard reference water sample N-45 (nutrients)

Definition of analytical methods, abbreviations, and symbols

Analytical methods

0. Other/Not reported

4. ICP

5. DCP

7. IC

$=$ inductively coupled plasma

= direct coupled plasma

20. Titrate: color

22. Color:

40. Ion electrode

$=$ ion chromatography

= titration: colonimetric (color reagent specified)

$=$ colorimetric [color reagent specified]

$=$ ion selective electrode

Abbreviations and symbols

$\begin{aligned} N= & \text { number of samples } \\ \text { St dev }= & \text { traditional standard deviation } \\ \text { MPV }= & \text { most probable value } \\ \text { F-pseudosigma }= & \text { nonparametric statistic deviation } \\ H u= & \text { upper hinge value } \\ H I= & \text { lower hinge value } \\ \mathrm{mg} / \mathrm{L}= & \text { milligrams per liter } \\ \text { Lab }= & \text { laboratory code number } \\ \mathrm{NR}= & \text { not rated, less than value reported } \\ <= & \text { less than }\end{aligned}$

Constituent

$\mathrm{NH} 3$ as $\mathrm{N}$

Ammonia as nitrogen

page

$\mathrm{NH} 3+$ Org $\mathrm{N}$ as $\mathrm{N}$

$\mathrm{NO} 3+\mathrm{NO} 2$ as $\mathrm{N}$

Ammonia plus organic nitrogen

B3

Total $\mathrm{P}$ as $\mathrm{P}$

Nitrate plus nitrite as nitrogen

84

$\mathrm{PO} 4$ as $\mathrm{P}$

Total Phosphorus as phosphorus

Orthophosphate as phosphorus 
Table 14. Statistical summary of reported data for standard reference water sample N-45 (nutrients)--Continued $\mathrm{NH3}$ as $\mathbf{N}$ (Ammonia) $\mathrm{mg} / \mathrm{l}$

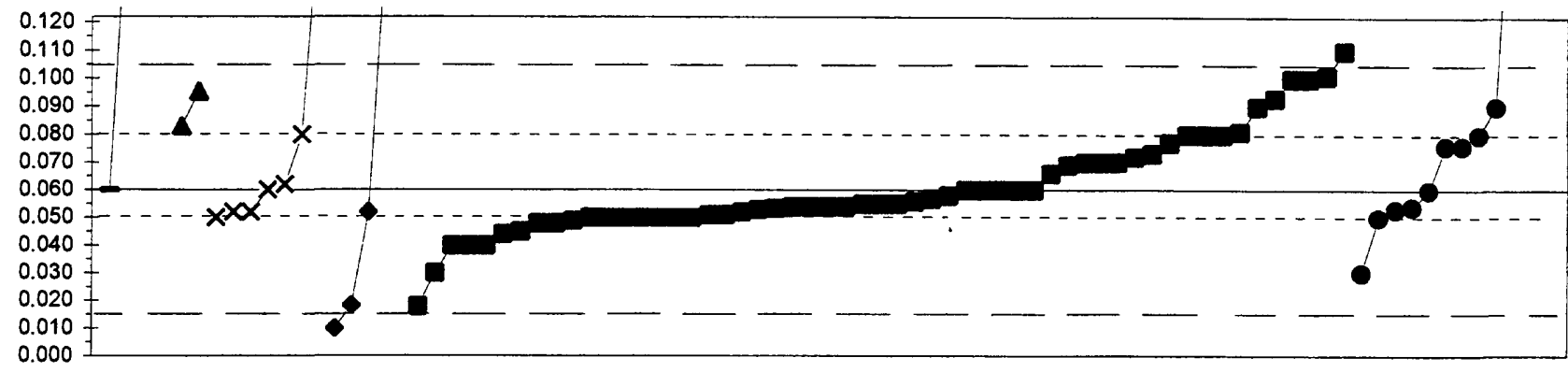

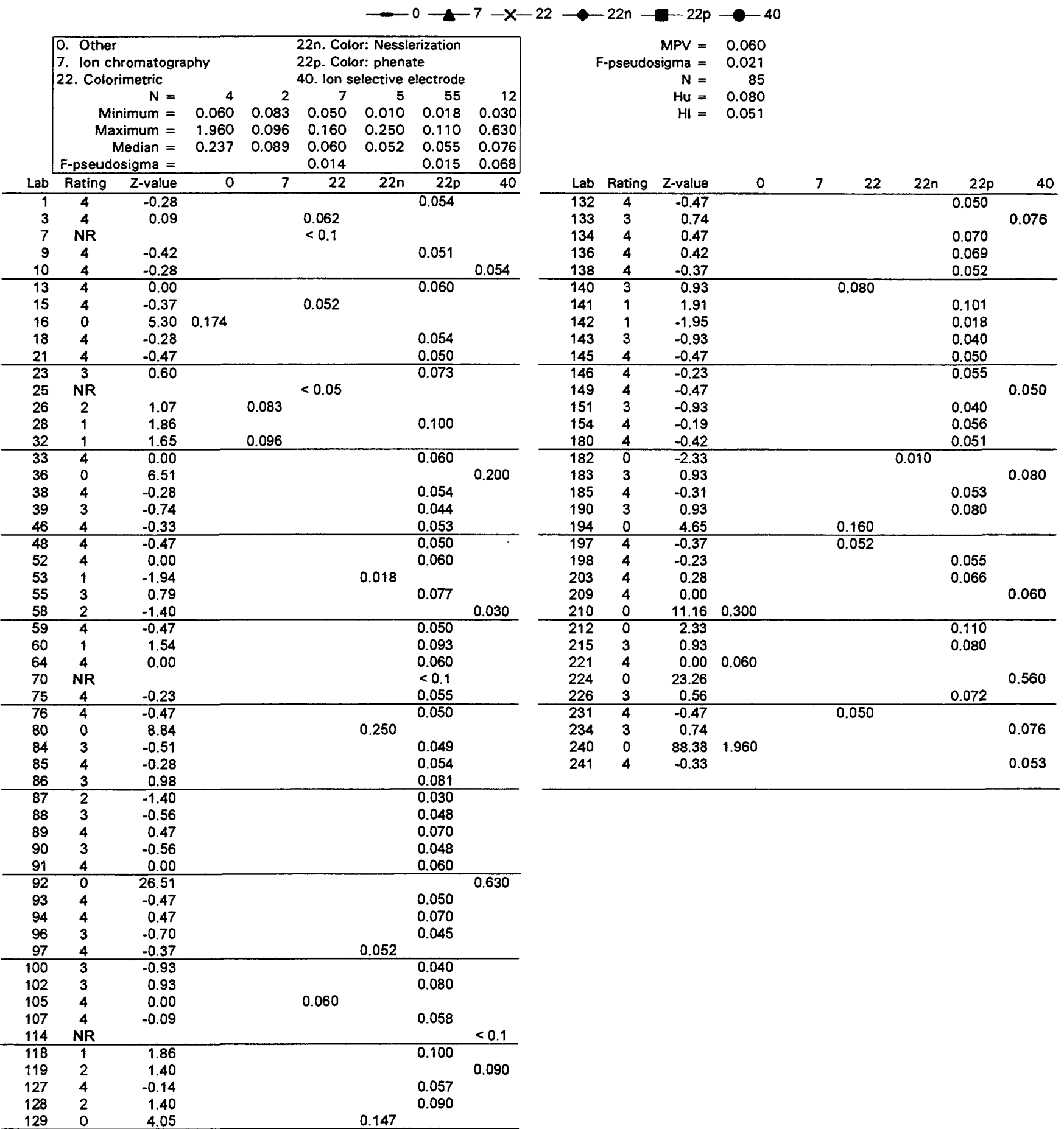


Table 14. Statistical summary of reported data for standard reference water sample N-45 (nutrients)--Continued NH3 + Org N as N (Ammonia + Organic N) $\mathrm{mg} / \mathrm{I}$

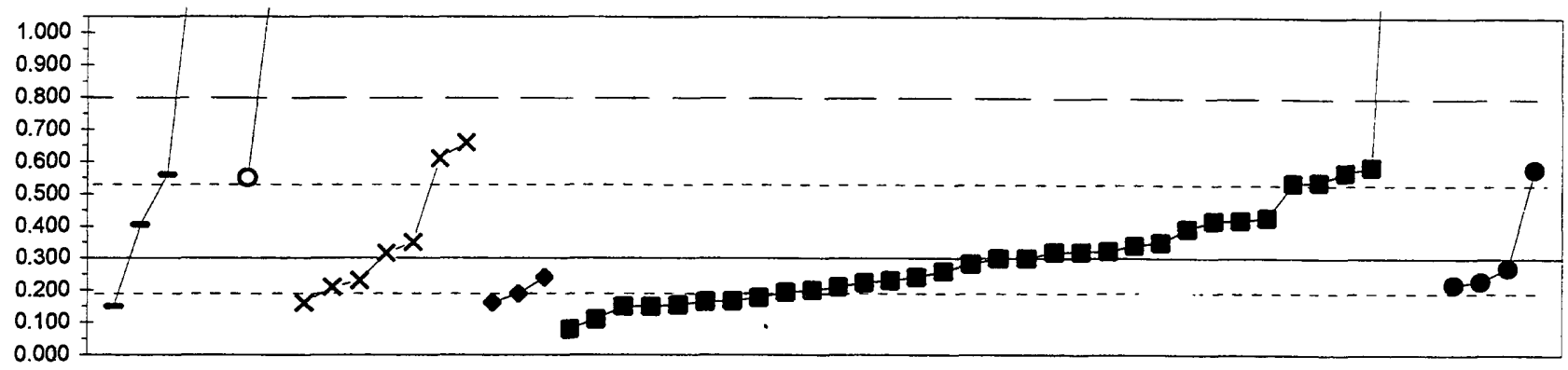

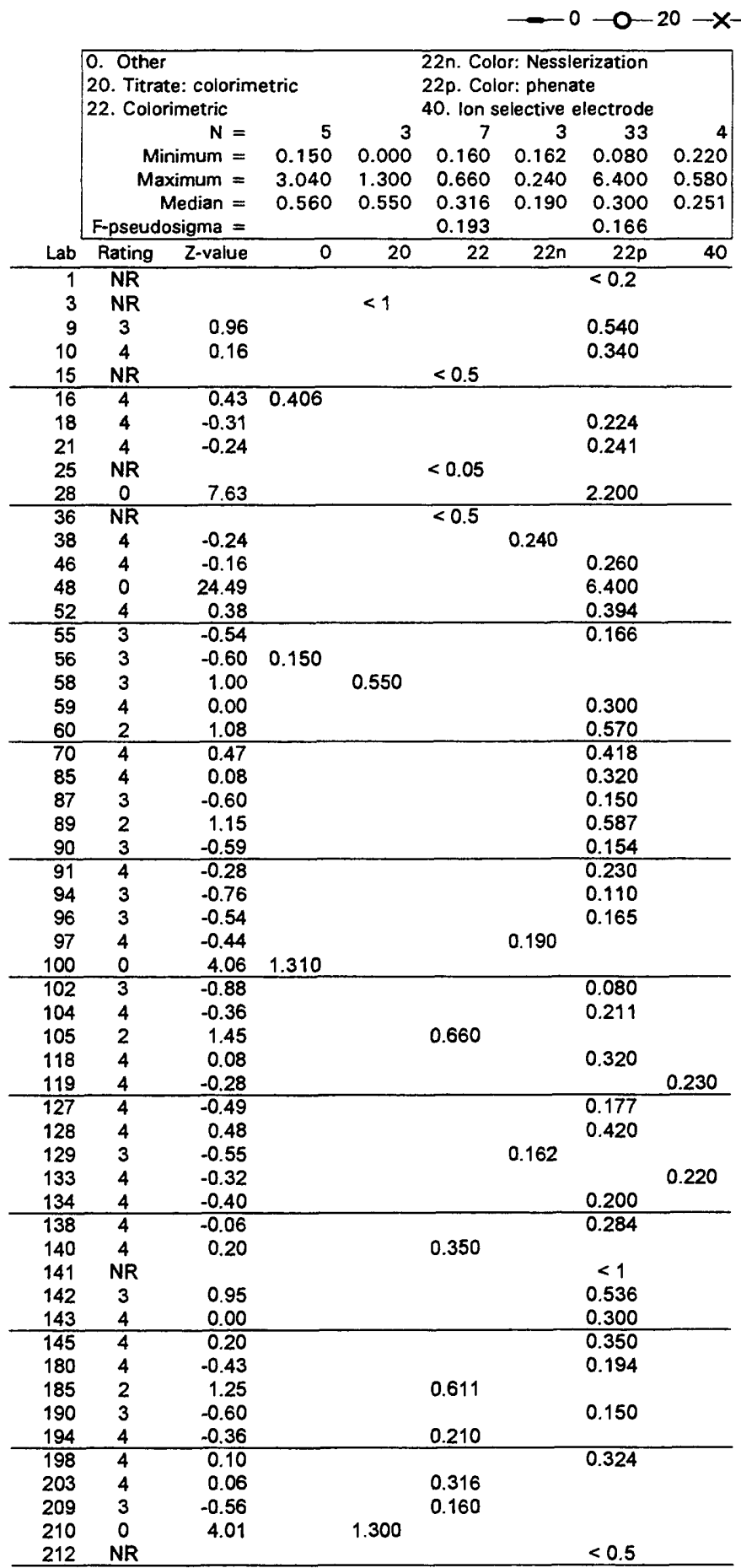


Table 14. Statistical summary of reported data for standard reference water sample N-45 (nutrients)--Continued $\mathrm{NO3}+\mathrm{NO2}$ as N (Nitrate + Nitrite) $\mathrm{mg} / \mathrm{l}$

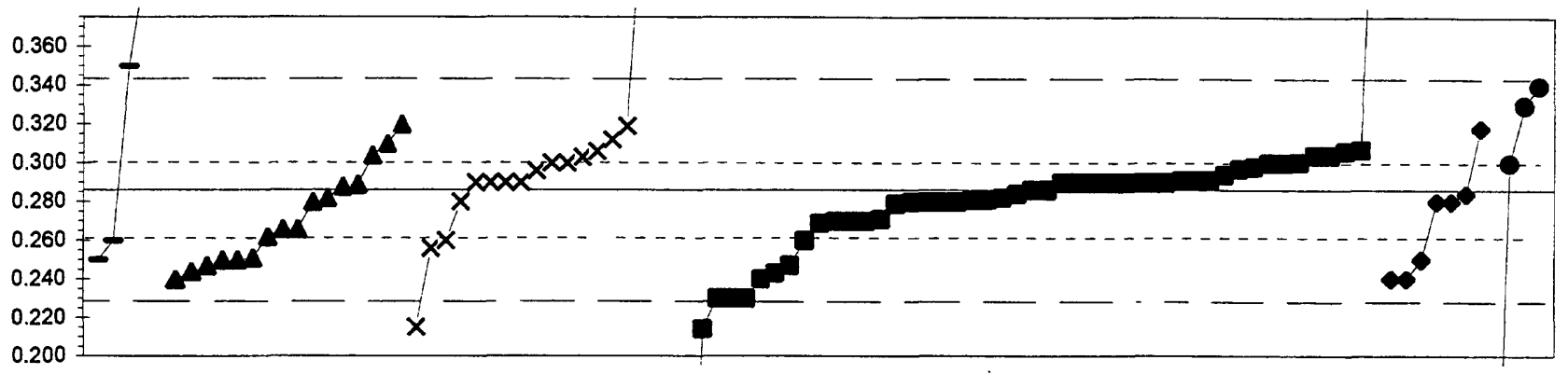

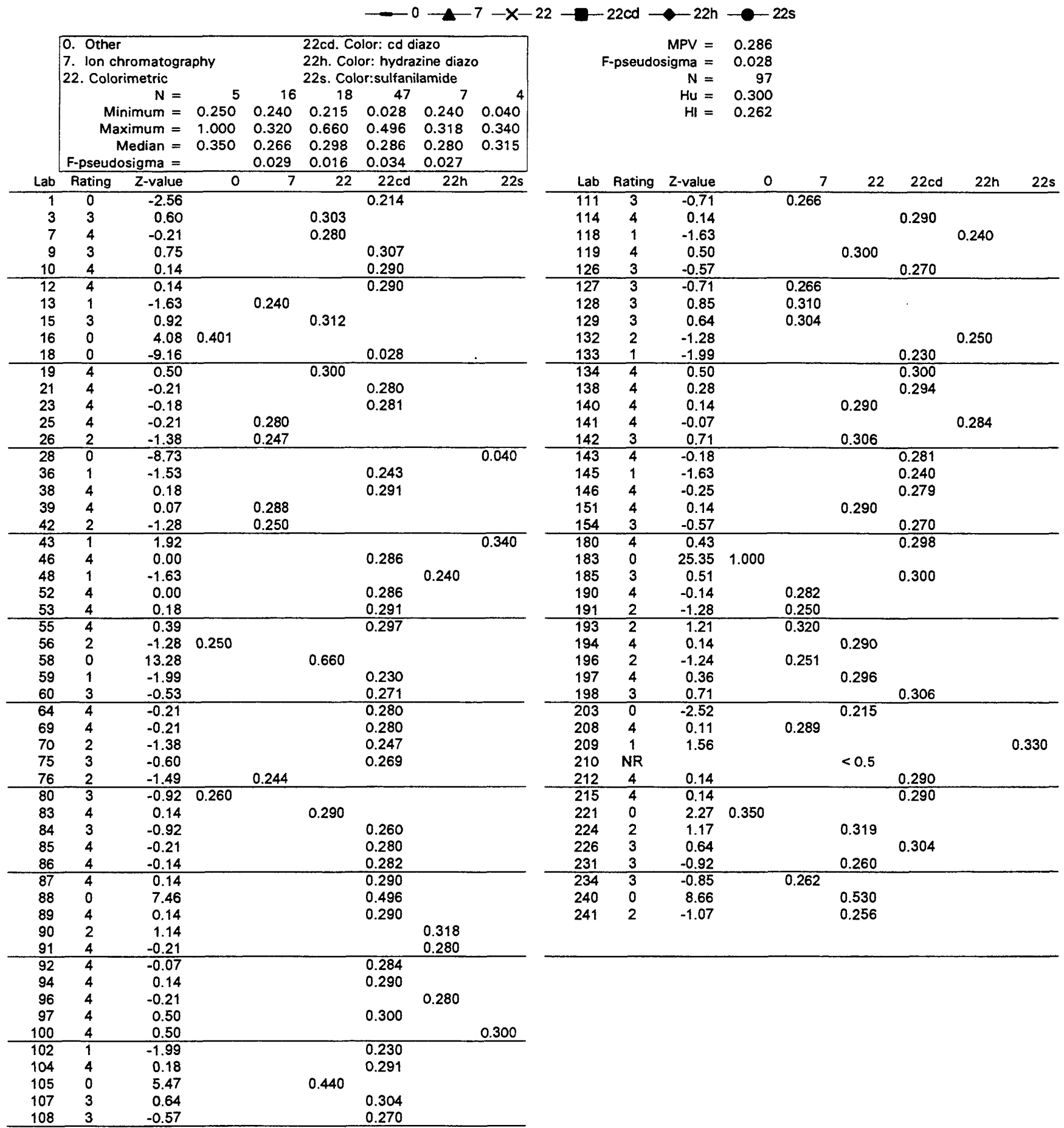


Table 14. Statistical summary of reported data for standard reference water sample $\mathrm{N}-45$ (nutrients)--Continued Total P as P (total Phosphorus) $\mathrm{mg} / \mathrm{l}$

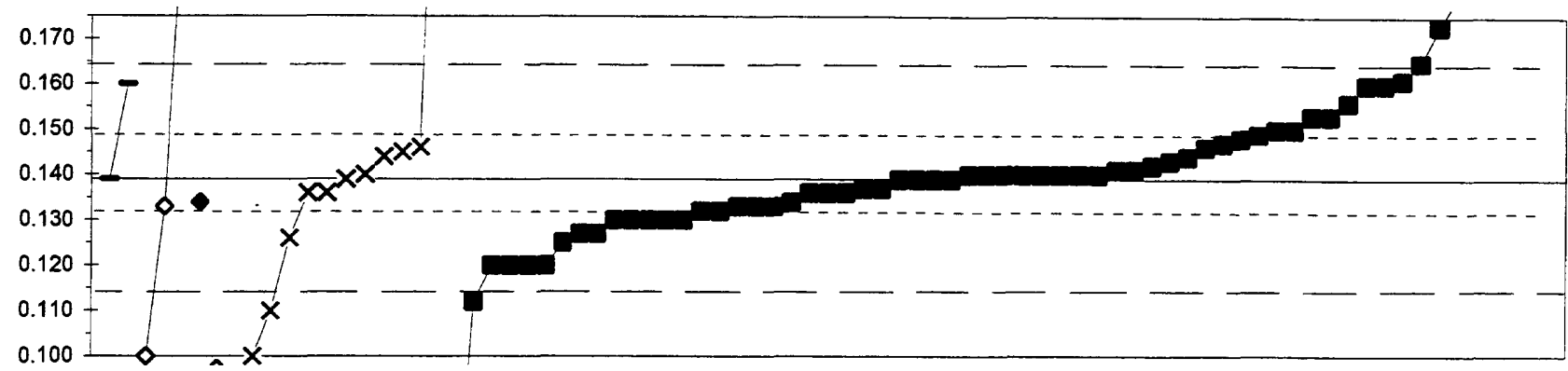

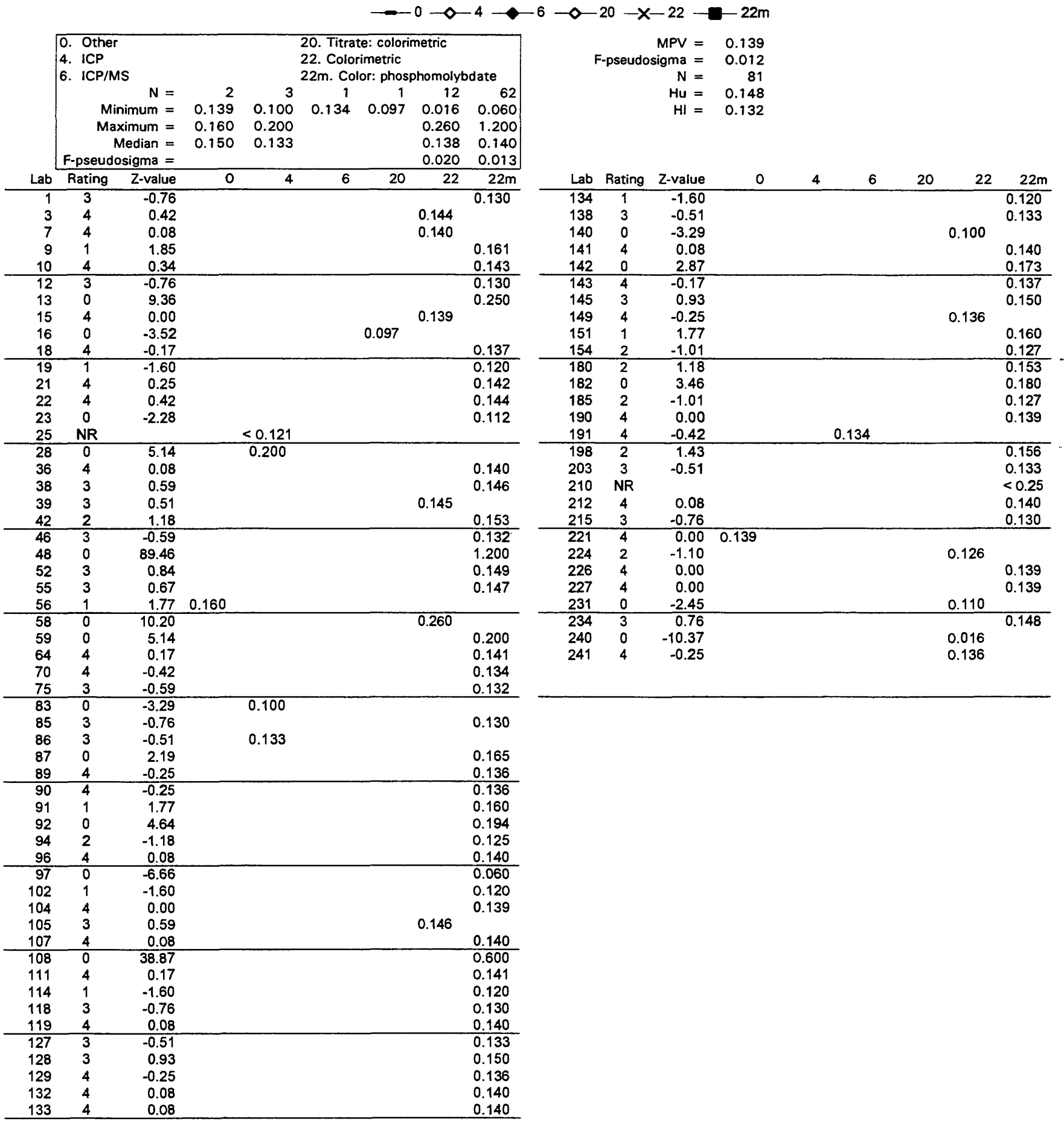


Table 14. Statistical summary of reported data for standard reference water sample N-45 (nutrients)--Continued PO4 as P (Orthophosphate) $\mathrm{mg} / \mathrm{l}$

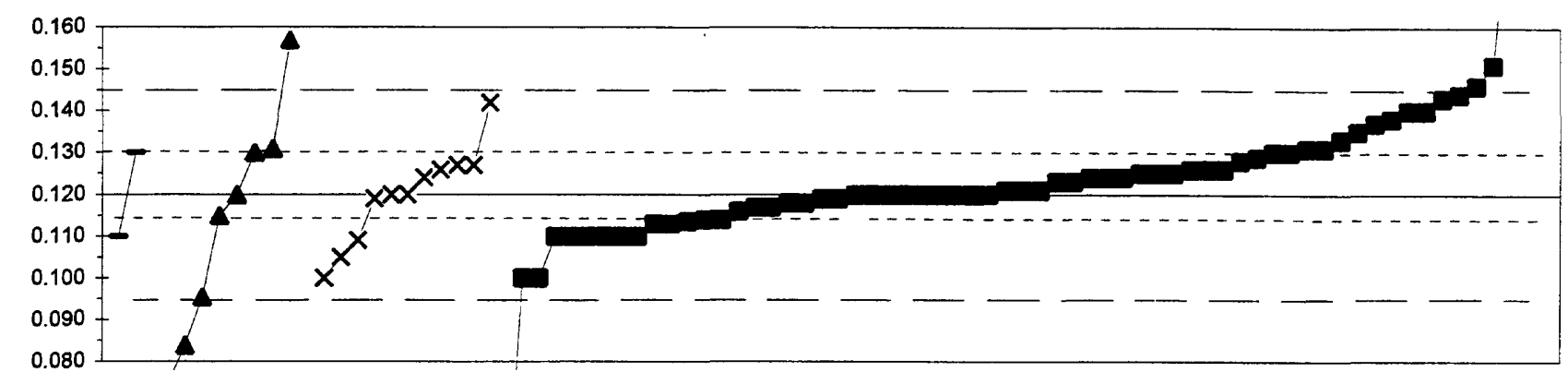

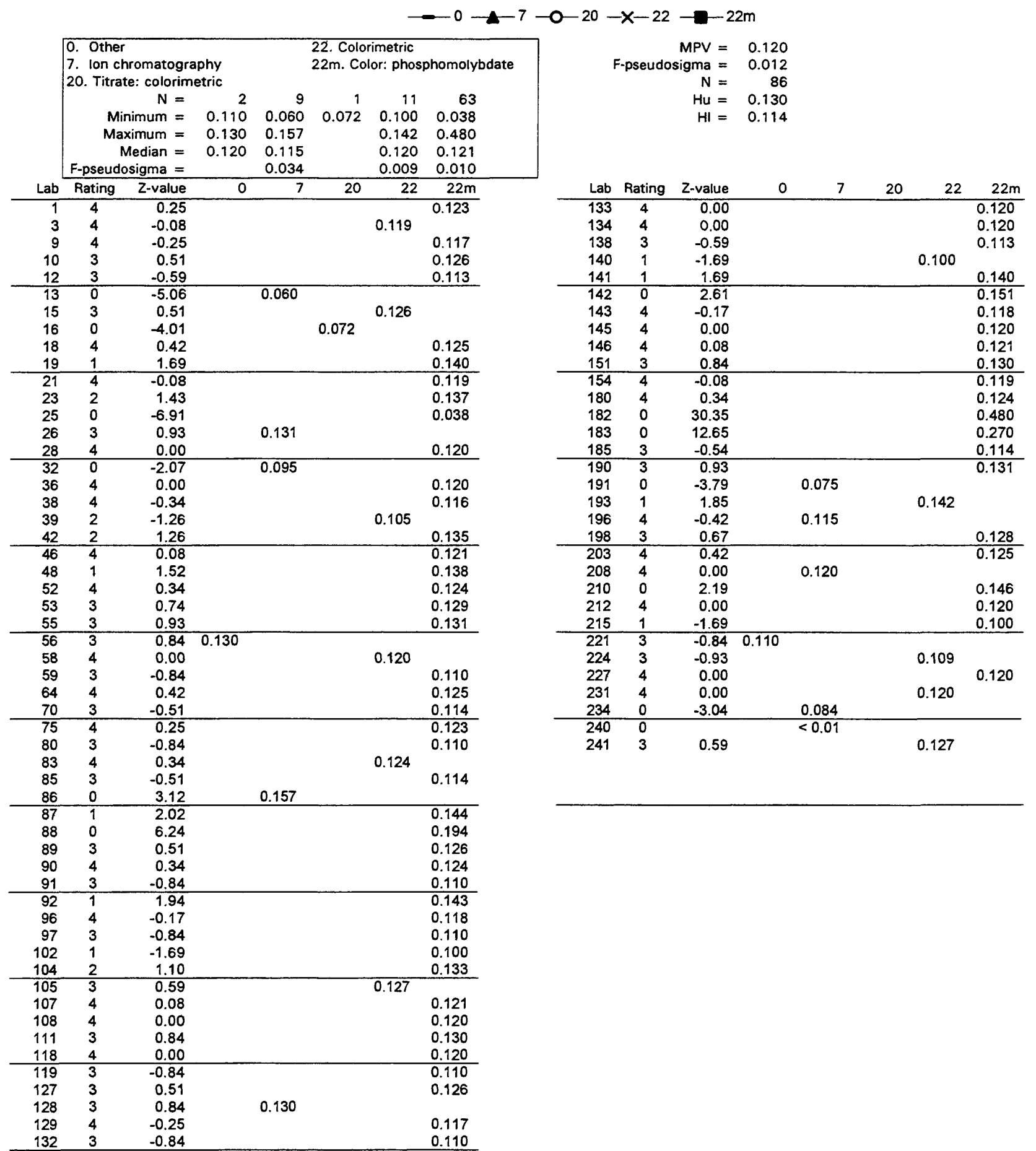


Table 15. Statistical summary of reported data for standard reference water sample $\mathrm{N}-46$ (nutrients)

Definition of analytical methods, abbreviations, and symbols

Analytical methods

0. Other/Not reported

4. ICP

5. DCP

7. IC

20. Titrate: color

22. Color:

40. Ion electrode

$=$ inductively coupled plasma

$=$ direct coupled plasma

$=$ ion chromatography

$=$ titration: colorimetric (color reagent specified)

$=$ colorimetric [color reagent specified]

$=$ ion selective electrode

Abbreviations and symbols

$\begin{aligned} N= & \text { number of samples } \\ \text { St dev }= & \text { traditional standard deviation } \\ \text { MPV }= & \text { most probable value } \\ \text { F-pseudosigma }= & \text { nonparametric statistic deviation } \\ H u= & \text { upper hinge value } \\ H I= & \text { lower hinge value } \\ \mathrm{mg} / \mathrm{L}= & \text { milligrams per liter } \\ \text { Lab }= & \text { laboratory code number } \\ \mathrm{NR}= & \text { not rated, less than value reported } \\ <= & \text { less than }\end{aligned}$

Constituent

$\mathrm{NH} 3$ as $\mathrm{N}$

Ammonia as nitrogen

$\mathrm{NH} 3+\operatorname{Org} \mathrm{N}$ as $\mathrm{N}$

Ammonia plus organic nitrogen

89

$\mathrm{NO} 3+\mathrm{NO} 2$ as $\mathrm{N}$

Nitrate plus nitrite as nitrogen

90

Total $P$ as $P$

Total Phosphorus as phosphorus

91

$\mathrm{PO} 4$ as $\mathrm{P}$

Orthophosphate as phosphorus 
Table 15. Statistical summary of reported data for standard reference watert sample N-46 (nutrients)--Continued NH3 as N (Ammonia) $\mathrm{mg} / \mathrm{l}$

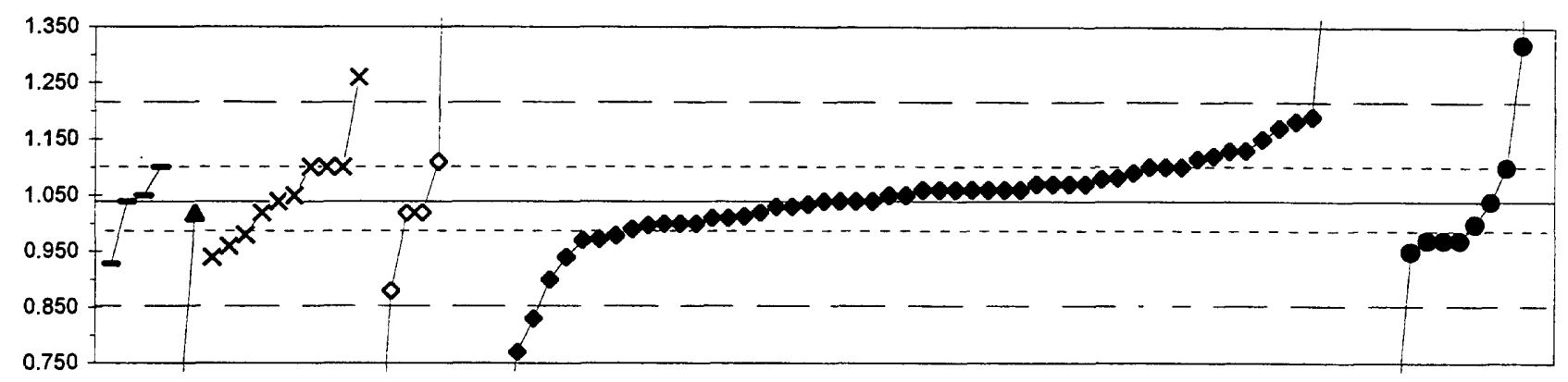

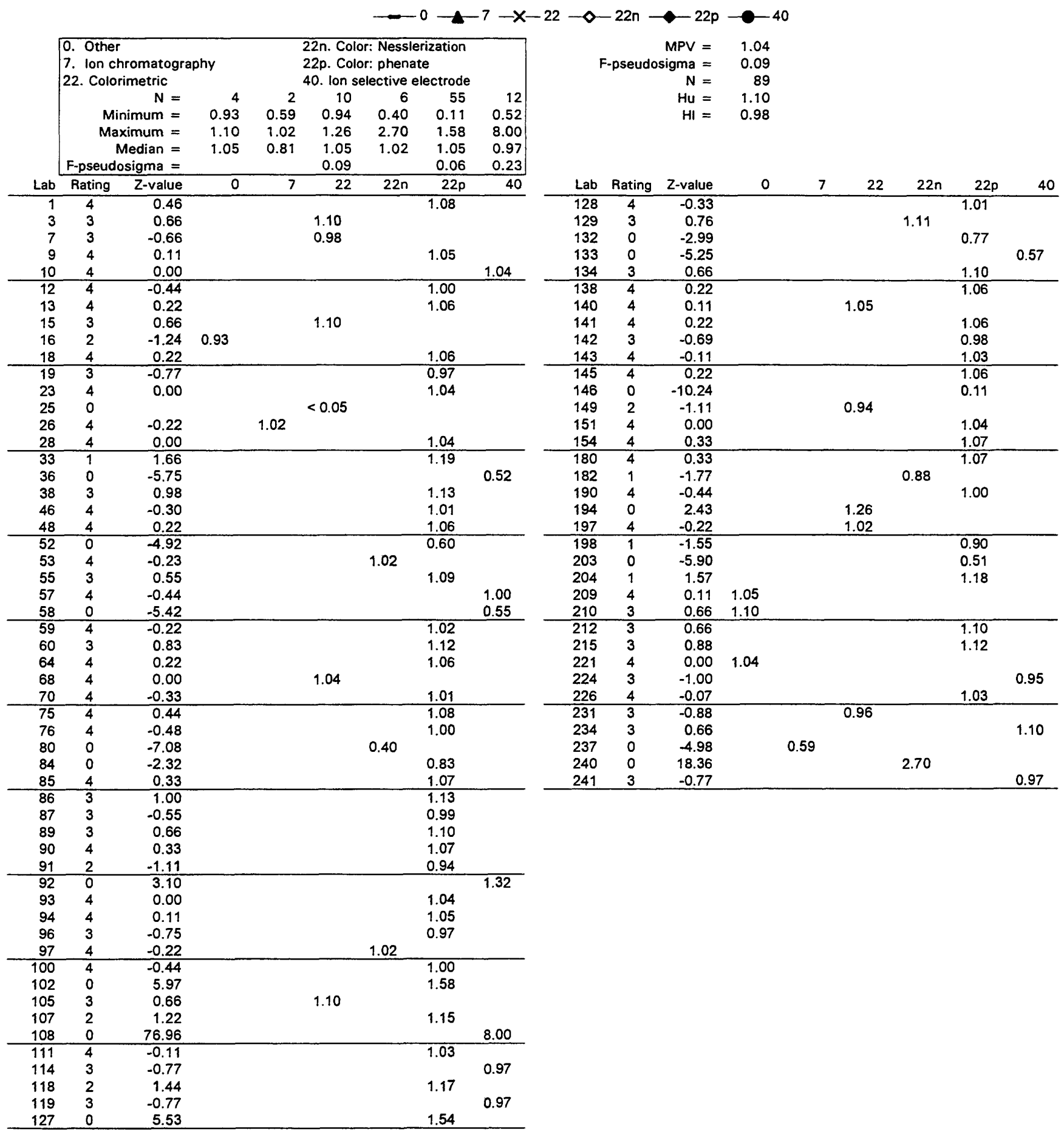


Table 15. Statistical summary of reported data for standard reference water sample N-46 (nutrients)--Continued $\mathbf{N H 3}+\operatorname{Org} \mathbf{N}$ as $\mathbf{N}$ (Ammonia + Organic N) $\mathrm{mg} / \mathrm{l}$

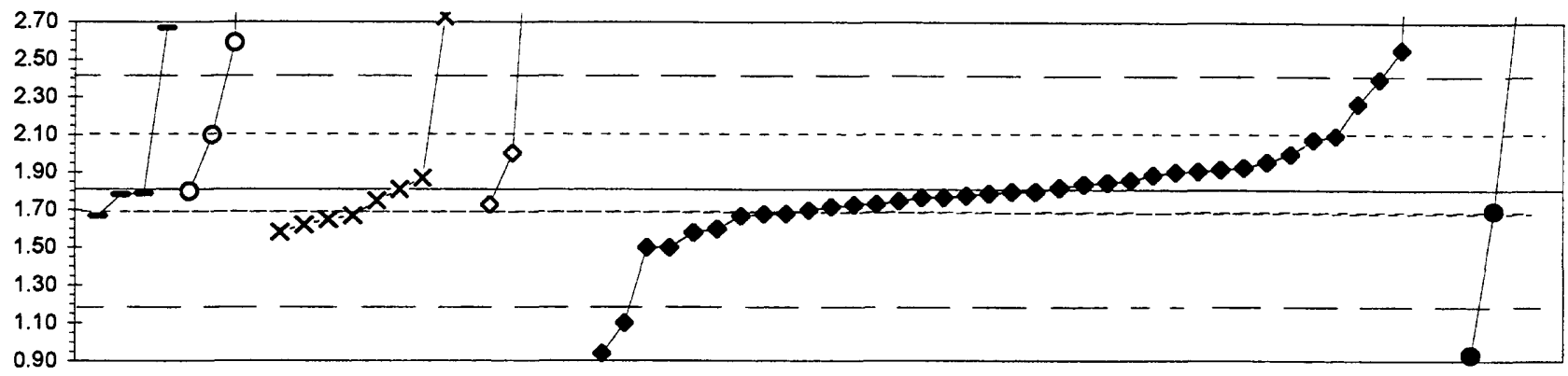

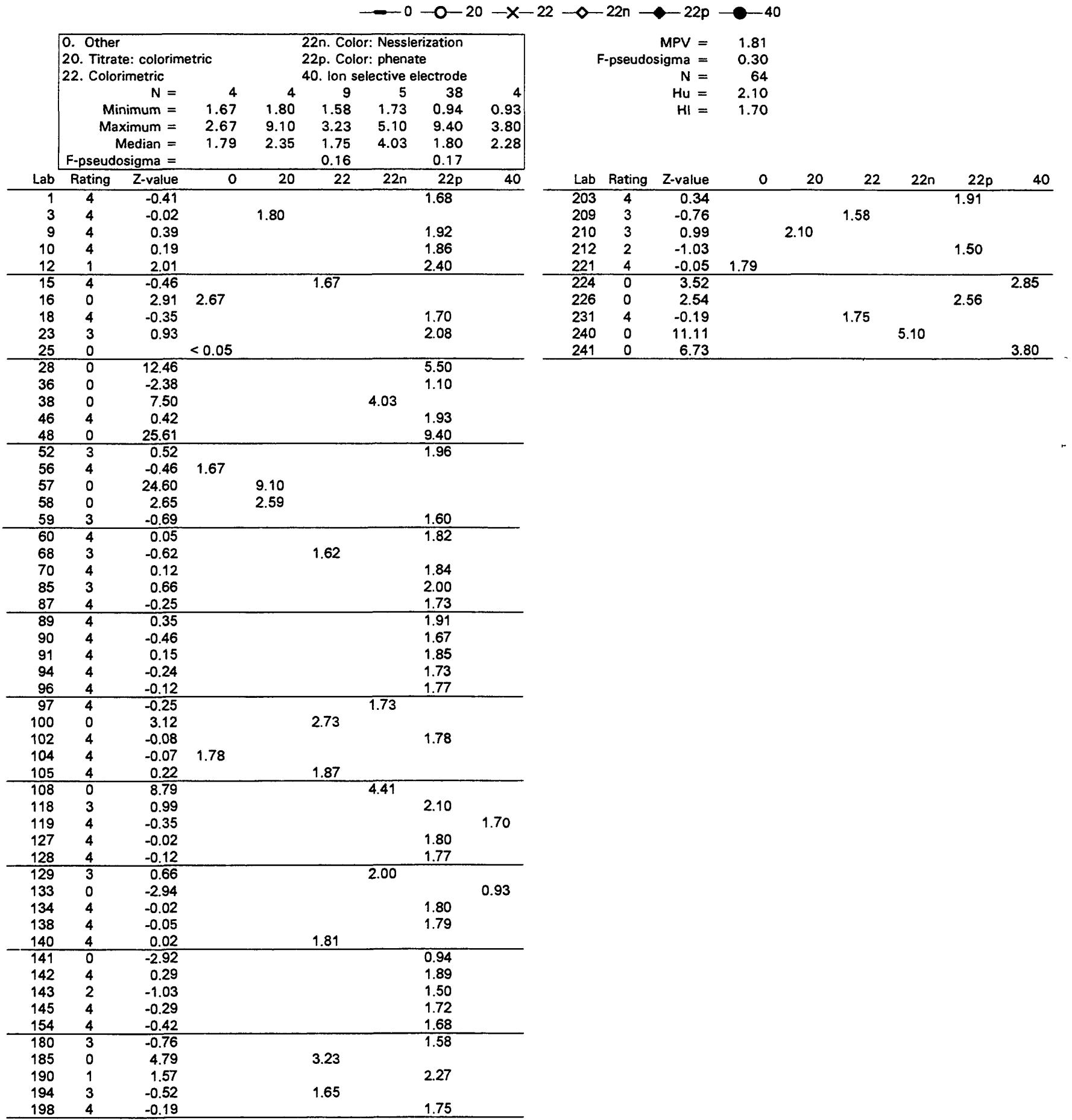


Table 15. Statistical summary of reported data for standard reference water sample $\mathrm{N}-46$ (nutrients)--Continued $\mathrm{NO} 3+$ NO2 as N (Nitrate + Nitrite) $\mathrm{mg} / \mathrm{l}$

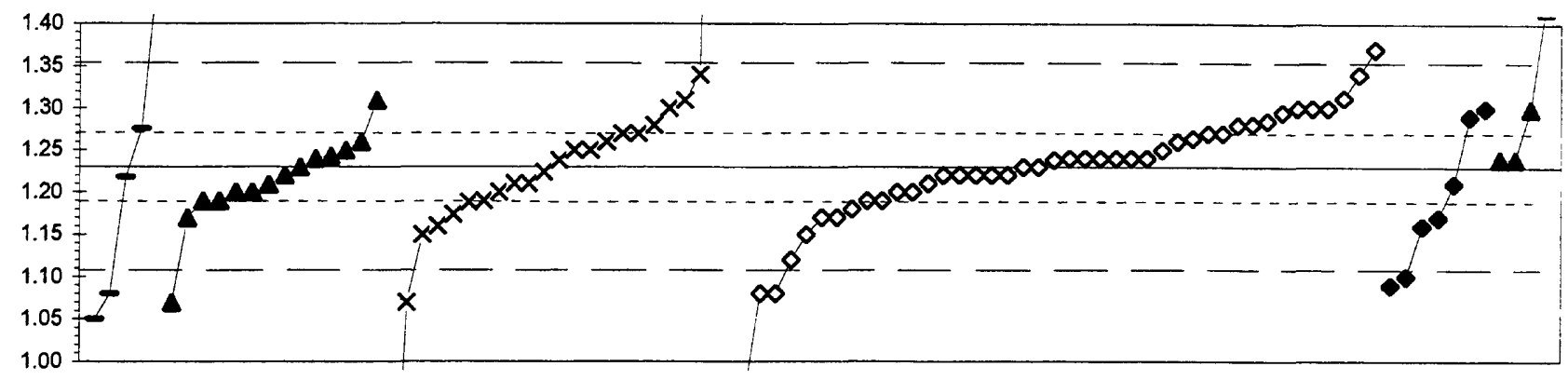

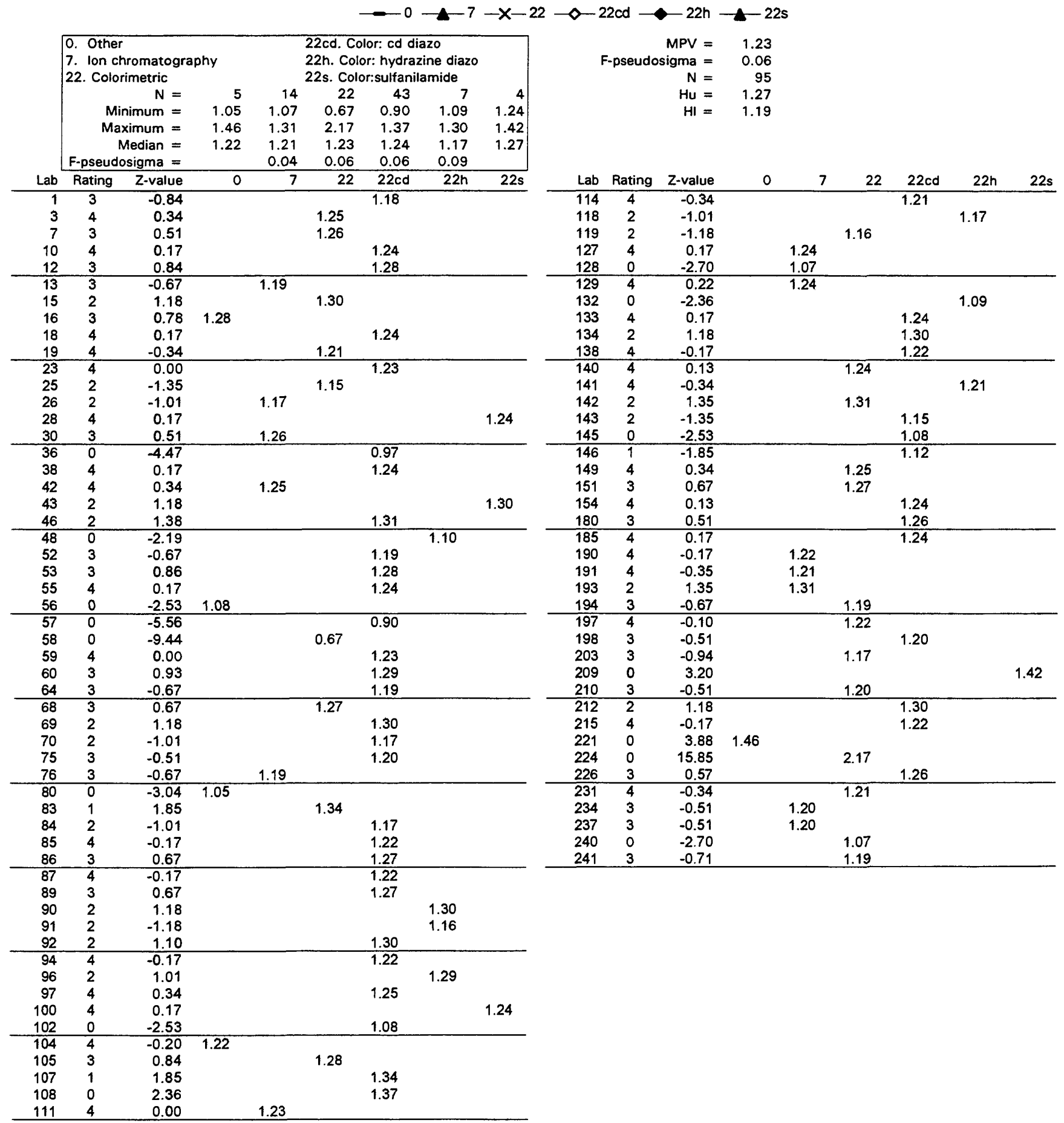


Table 15. Statistical summary of reported data for standard reference water sample N-46 (nutrients)--Continued

\section{Total P as P (total Phosphorus) $\mathrm{mg} / \mathrm{l}$}

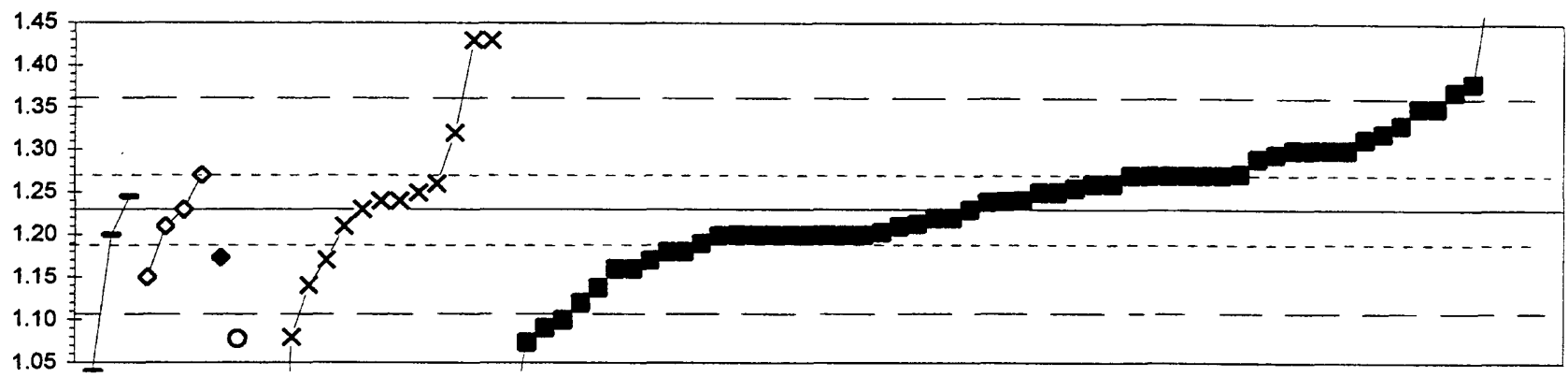

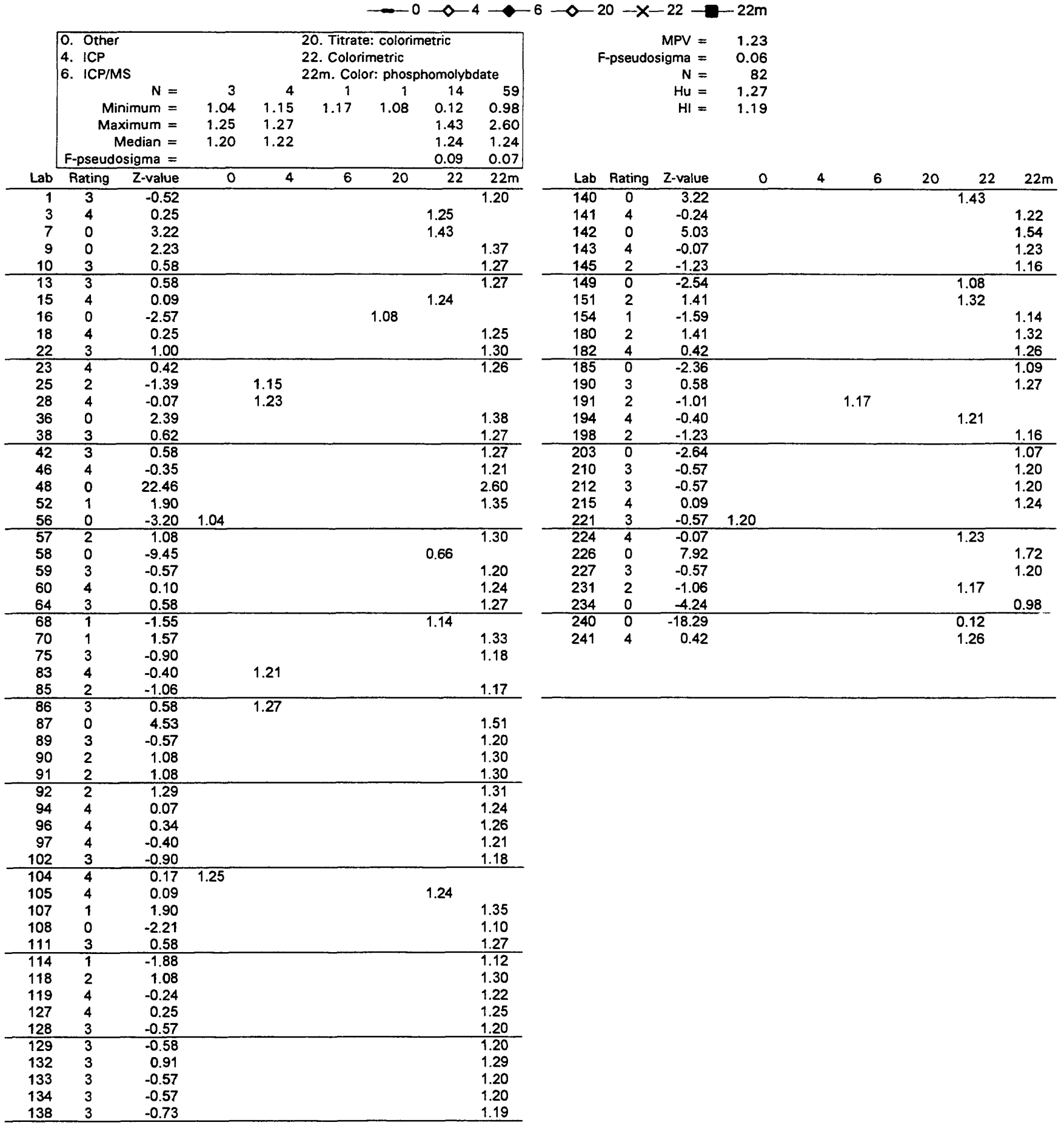


Table 15. Statistical summary of reported data for standard reference water sample N-46 (nutrients)--Continued PO4 as P (Orthophosphate) $\mathrm{mg} / \mathrm{l}$

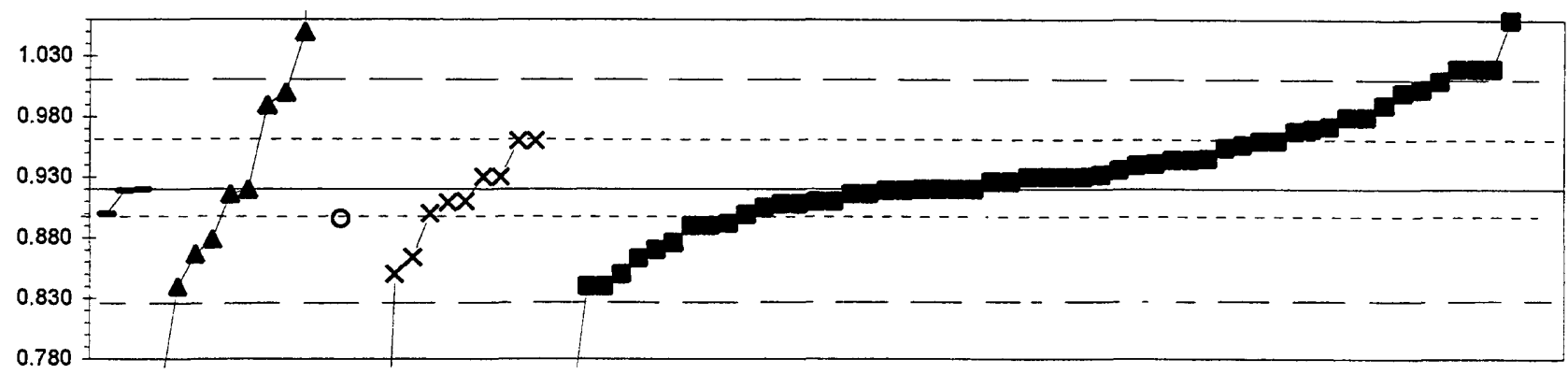

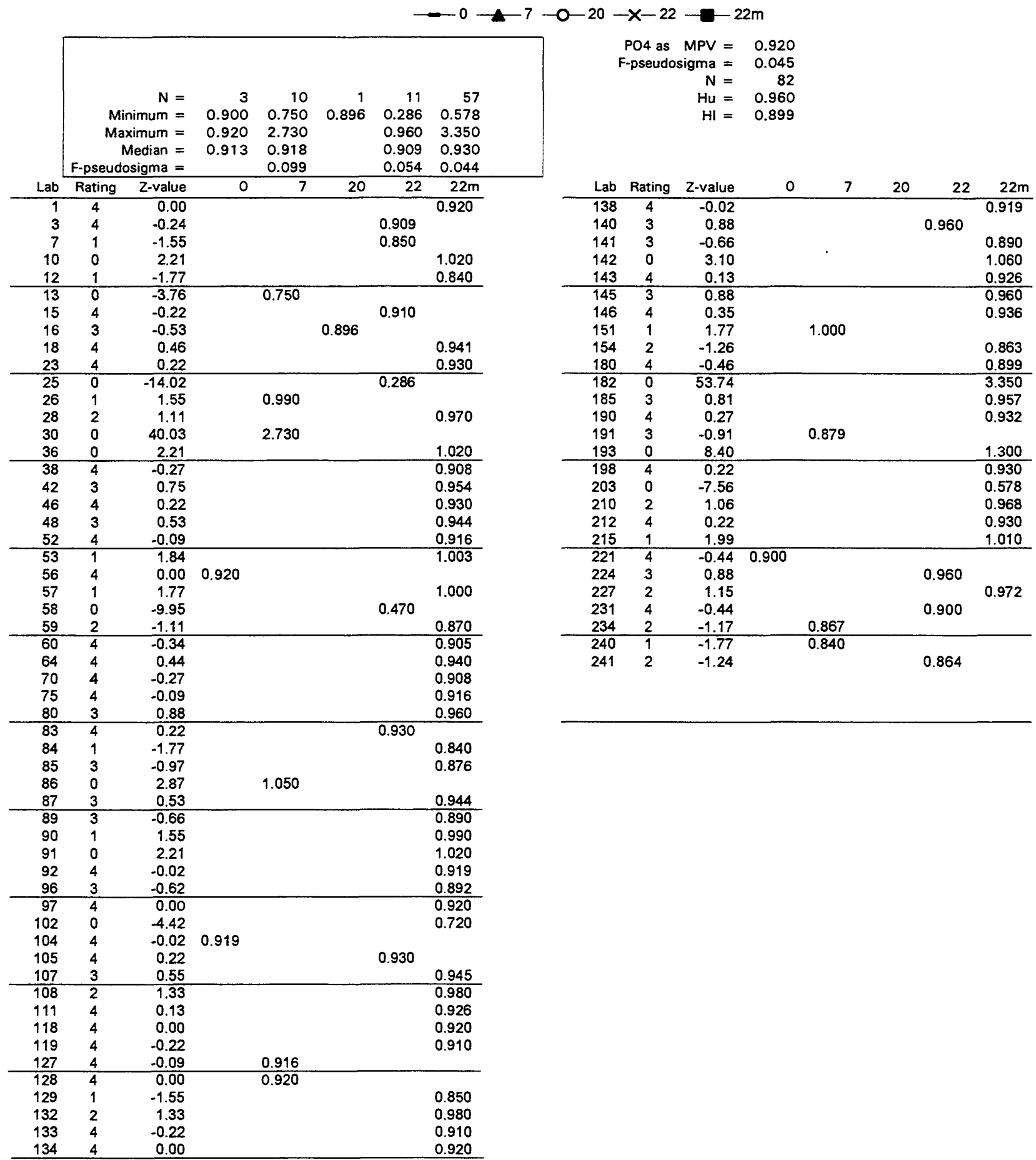


Table 16. Statistical summary of reported data for standard reference water sample P-24 (low ionic strength)

Definition of analytical methods, abbreviations, and symbols

Analytical methods

0 Other/Not reported

1 AA: direct, air

2 AA: direct, N2O

3 AA: graphite fumace

4 ICP

5 DCP

6 ICP/MS

7 IC

12 Flame emission

20 Titrate: color

21 Titrate: electro

22 Color.

40 lon electrode

41 Electro

50 Gravimetric

51 Turbidimetric

= atomic absorption: direct,air

$=$ atomic absorption: direct, nitrous oxide

= atomic absorption: graphite furnace

$=$ inductively coupled plasma

= direct current plasma

= mass spectrometry/inductively coupled plasma

$=$ ion chromatography

= flame emission

= titration: colonimetric [color reagent specified]

titration: electrometric

colorimetric [color reagent specified]

$=$ ion selective electrode

$=$ electrometric: [type meter specified]

= gravimetric: [precipitate specified]

$=$ turbidimetric: [precipitate specified]

Abbreviations and symbols

\begin{tabular}{|c|c|}
\hline$N=$ & number of samples \\
\hline St dev $=$ & traditional standard deviation \\
\hline MPV $=$ & most probable value \\
\hline pseudosigma $=$ & nonparametric statistic deviation \\
\hline $\mathrm{Hu}=$ & upper hinge value \\
\hline $\mathrm{HI}=$ & lower hinge value \\
\hline $\mathrm{mg} / \mathrm{L}=$ & milligrams per liter \\
\hline$\mu \mathrm{S} / \mathrm{cm}=$ & microsiemens per centimeter at $25 \mathrm{C}$ \\
\hline$L a b=$ & laboratory code number \\
\hline $\begin{array}{r}\mathrm{NR}= \\
<=\end{array}$ & $\begin{array}{l}\text { not rated, less than value reported } \\
\text { less than }\end{array}$ \\
\hline
\end{tabular}

Constituent

Acid Acidity as $\mathrm{CaCO} 3$

page

95

$\mathrm{Ca} \quad$ Calcium

96

Cl Chloride

97

$F \quad$ Fluoride

98

K Potassium

99

Mg Magnesium 100

$\mathrm{Na}$ Sodium 101

$\mathrm{pH} \quad 102$

PO4 as P Orthophosphate as Phosphorus 103

$\mathrm{SO} 4$ Sulfate 104

Sp Cond Specific Conductance 105 
Table 16. Statistical summary of reported data for standard reference sediment sample P-24 (low ionic strength)--Continued Acidity as $\mathrm{CaCO} 3$ $\mathrm{mg} / \mathrm{l}$

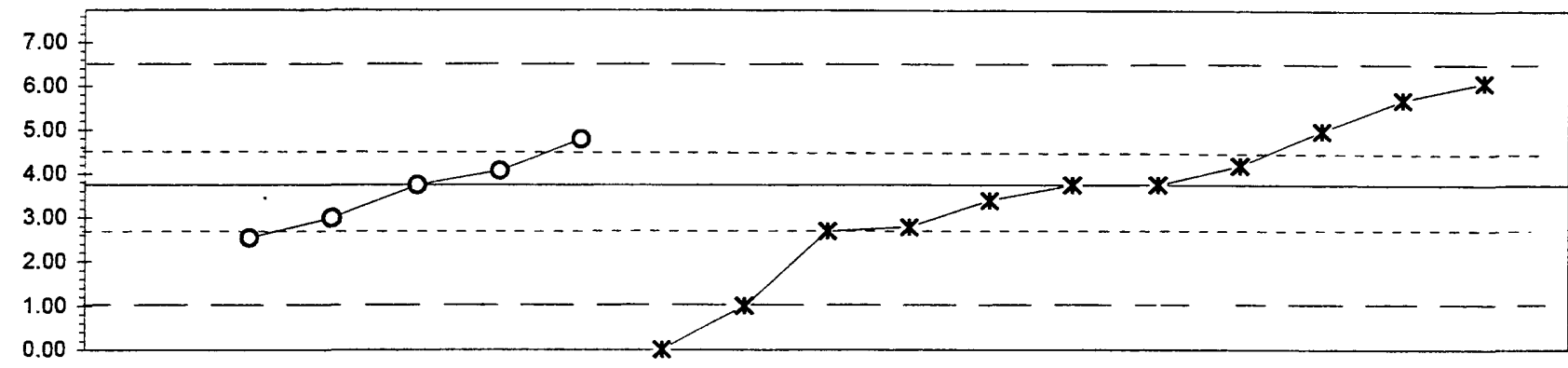

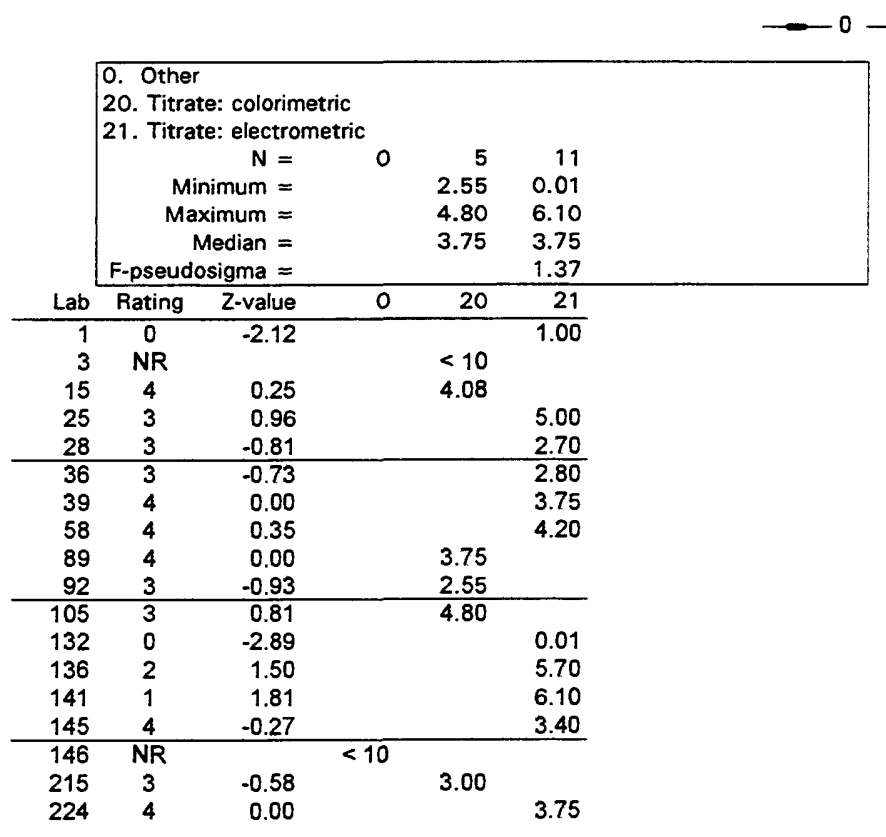


Table 16. Statistical summary of reported data for standard reference water sample P-24 (low ionic strength)--Continued Ca (Calcium) $\mathrm{mg} / \mathrm{l}$

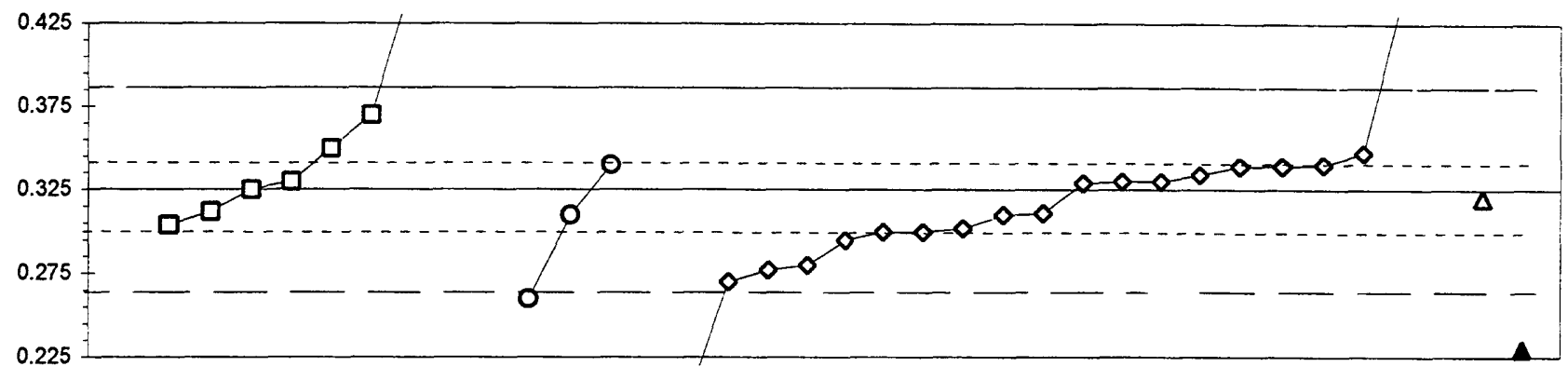

\begin{tabular}{|c|c|c|c|c|c|c|c|c|}
\hline \multirow[b]{7}{*}{ Lab } & \multicolumn{3}{|c|}{$\begin{array}{l}\text { 0. Other } \\
\text { 1. AA: direct air } \\
\text { 2. AA: direct nitrous oxide }\end{array}$} & \multicolumn{4}{|c|}{$\begin{array}{l}\text { 4. ICP } \\
\text { 5. DCP } \\
\text { 7. Ion chromatography }\end{array}$} & \multirow{6}{*}{$\begin{array}{r}1 \\
0.230\end{array}$} \\
\hline & \multirow{5}{*}{\multicolumn{2}{|c|}{$\begin{aligned} \text { Minimum } & = \\
\text { Maximum } & = \\
\text { Median } & = \\
\text { F-pseudosigma } & =\end{aligned}$}} & & \multirow{5}{*}{$\begin{array}{r}9 \\
0.304 \\
3.330 \\
0.350 \\
0.093 \\
\end{array}$} & \multirow{5}{*}{$\begin{array}{r}3 \\
0.260 \\
0.340 \\
0.310\end{array}$} & \multirow{5}{*}{$\begin{array}{l}21 \\
0.200 \\
0.580 \\
0.311 \\
0.033\end{array}$} & \multirow{5}{*}{$\begin{array}{r}{ }^{1} \\
0.320\end{array}$} & \\
\hline & & & & & & & & \\
\hline & & & & & & & & \\
\hline & & & & & & & & \\
\hline & & & & & & & & \\
\hline & Rating & $\mathrm{Z}$-value & 0 & 1 & 2 & 4 & 5 & 7 \\
\hline 1 & 4 & 0.17 & & 0.330 & & & & \\
\hline 3 & 4 & -0.47 & & & & 0.311 & & \\
\hline 11 & 3 & -0.51 & & & & 0.310 & & \\
\hline 15 & 3 & -0.78 & & & & 0.302 & & \\
\hline 23 & 0 & & & $<2$ & & & & \\
\hline 25 & 0 & -4.22 & & & & 0.200 & & \\
\hline 26 & 3 & 0.51 & & & & 0.340 & & \\
\hline 28 & 3 & -0.84 & & & & 0.300 & & \\
\hline 33 & 4 & -0.17 & & & & & 0.320 & \\
\hline 36 & NR & & & & $<0.5$ & & & \\
\hline 38 & 3 & 0.51 & & & 0.340 & & & \\
\hline 39 & 2 & -1.01 & & & & 0.295 & & \\
\hline 48 & 1 & -1.85 & & & & 0.270 & & \\
\hline 52 & 4 & 0.30 & & & & 0.334 & & \\
\hline 58 & 0 & 4.22 & & 0.450 & & & & \\
\hline 64 & 0 & 101.34 & & 3.330 & & & & \\
\hline 81 & 1 & -1.62 & & & & 0.277 & & \\
\hline 89 & 3 & -0.71 & & 0.304 & & & & \\
\hline 92 & 0 & 86.84 & & 2.900 & & & & \\
\hline 101 & 3 & 0.84 & & 0.350 & & & & \\
\hline 102 & 0 & -4.22 & & & & 0.200 & & \\
\hline 105 & 4 & 0.13 & & & & 0.329 & & \\
\hline 111 & 3 & -0.51 & & & 0.310 & & & \\
\hline 132 & 0 & 4.05 & & & & 0.445 & & \\
\hline 134 & 4 & 0.47 & & & & 0.339 & & \\
\hline 136 & 0 & -2.19 & & & 0.260 & & & \\
\hline 138 & 3 & 0.74 & & & & 0.347 & & \\
\hline 140 & 4 & 0.00 & & 0.325 & & & & \\
\hline 141 & 1 & -1.52 & & & & 0.280 & & \\
\hline 145 & 3 & -0.84 & & & & 0.300 & & \\
\hline 146 & NR & & $<0.5$ & & & & & \\
\hline 180 & 4 & 0.17 & & & & 0.330 & & \\
\hline 190 & 0 & -3.20 & & & & & & 0.230 \\
\hline 194 & NR & & & & & $<5$ & & \\
\hline 196 & 1 & 1.52 & & 0.370 & & & & \\
\hline 209 & 4 & & & & & 0.330 & & \\
\hline 215 & 0 & 8.60 & & & & 0.580 & & \\
\hline 221 & 4 & -0.44 & & 0.312 & & & & \\
\hline 224 & 4 & 0.48 & & & & 0.339 & & \\
\hline
\end{tabular}


Table 16. Statistical summary of reported data for standard reference water sample P-24 (low ionic strength)--Continued Cl (Chloride)

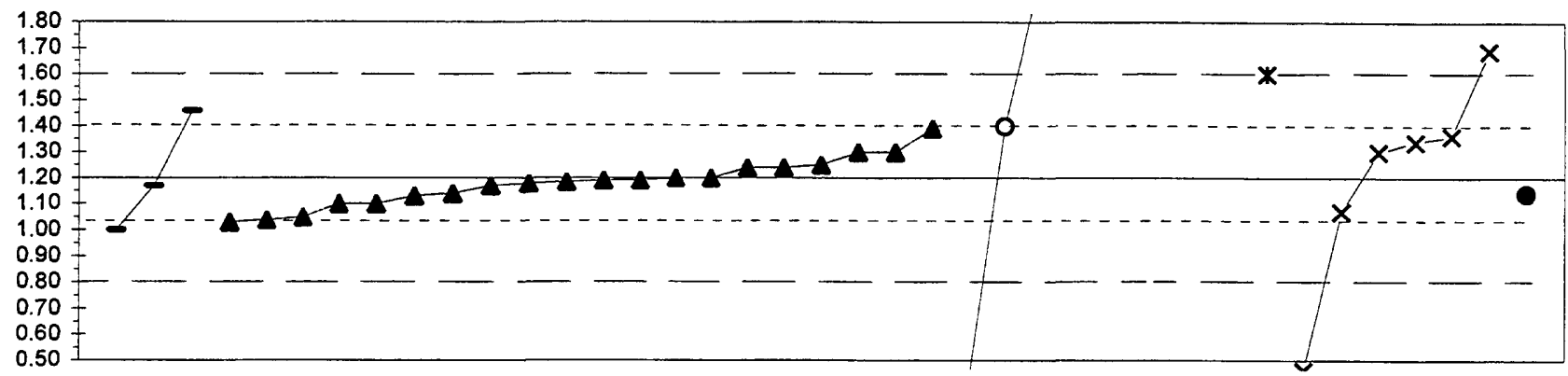

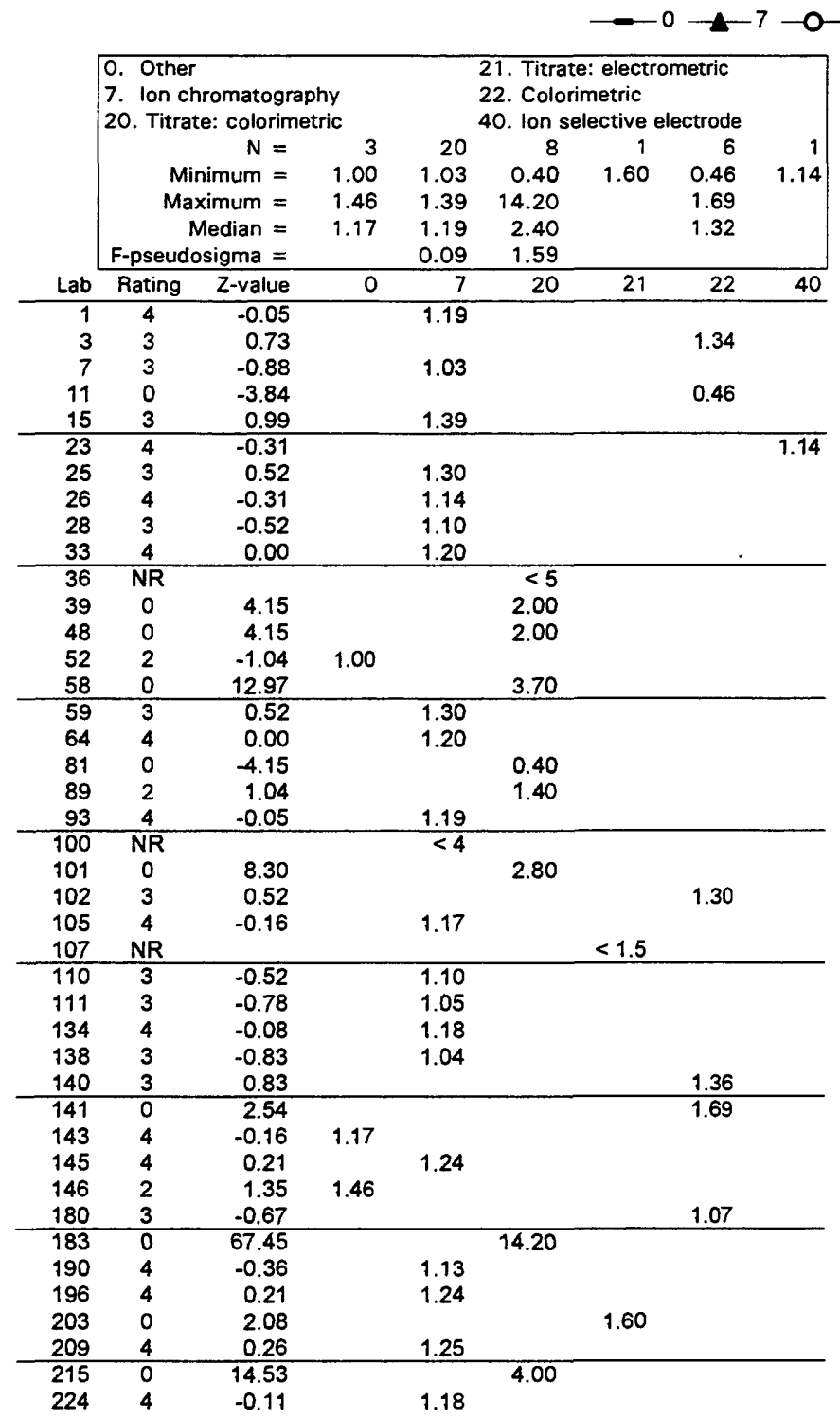


Table 16. Statistical summary of reported data for standard reference water sample P-24 (low ionic strength)--Continued $F$ (Fluoride) $\mathrm{mg} / \mathrm{l}$

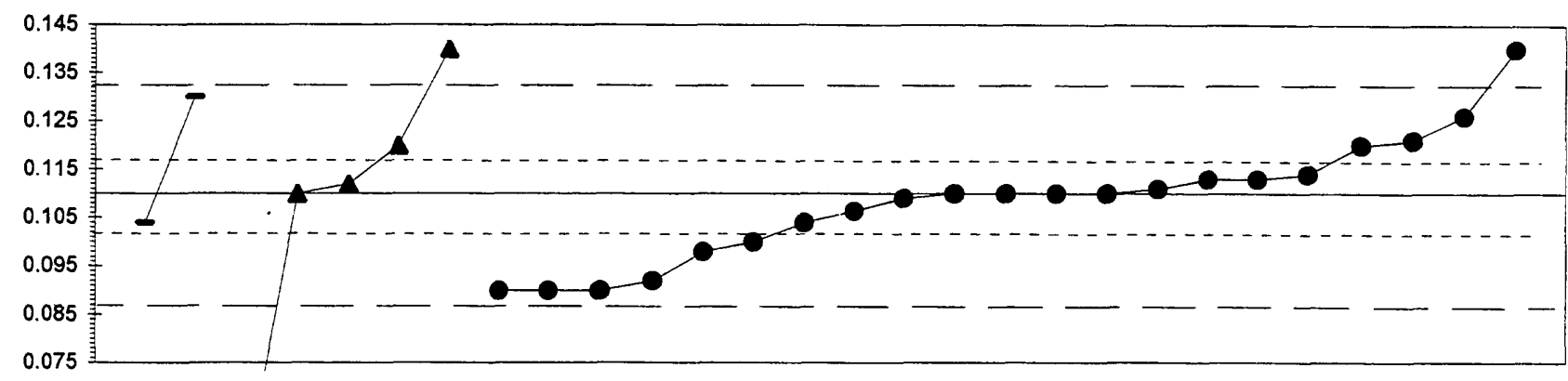

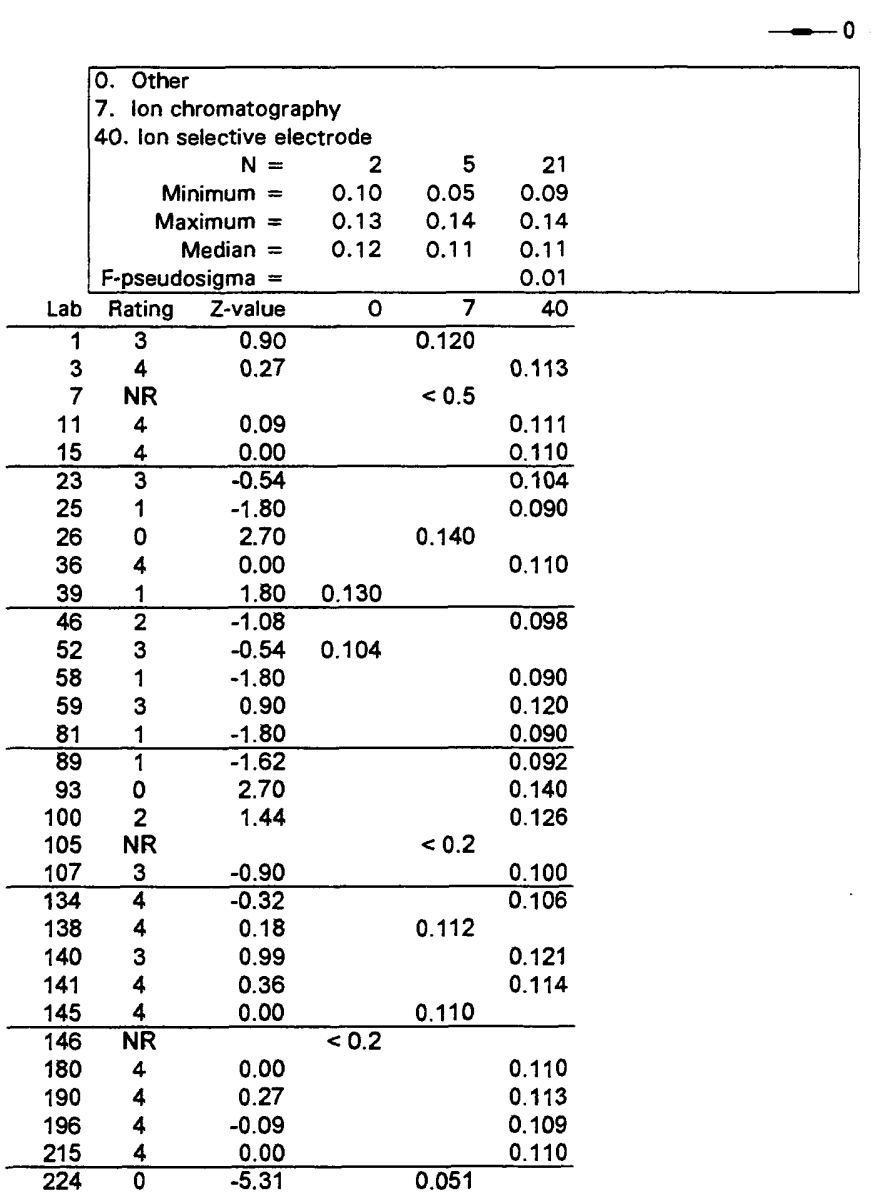


Table 16. Statistical summary of reported data for standard reference water sample P-24 (low ionic strength)--Continued K (Potassium) $\mathrm{mg} / \mathrm{l}$

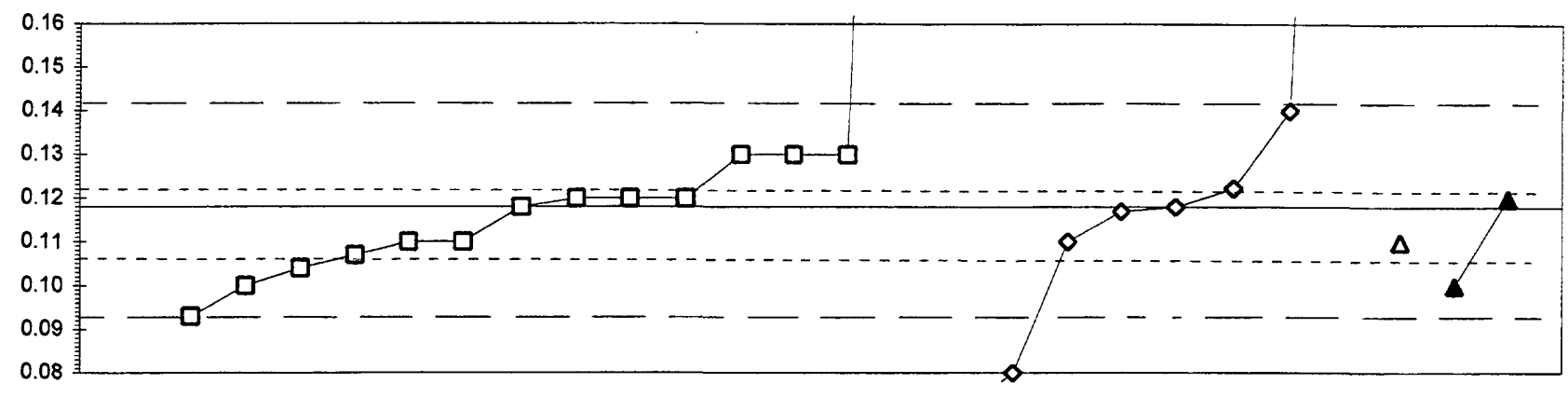

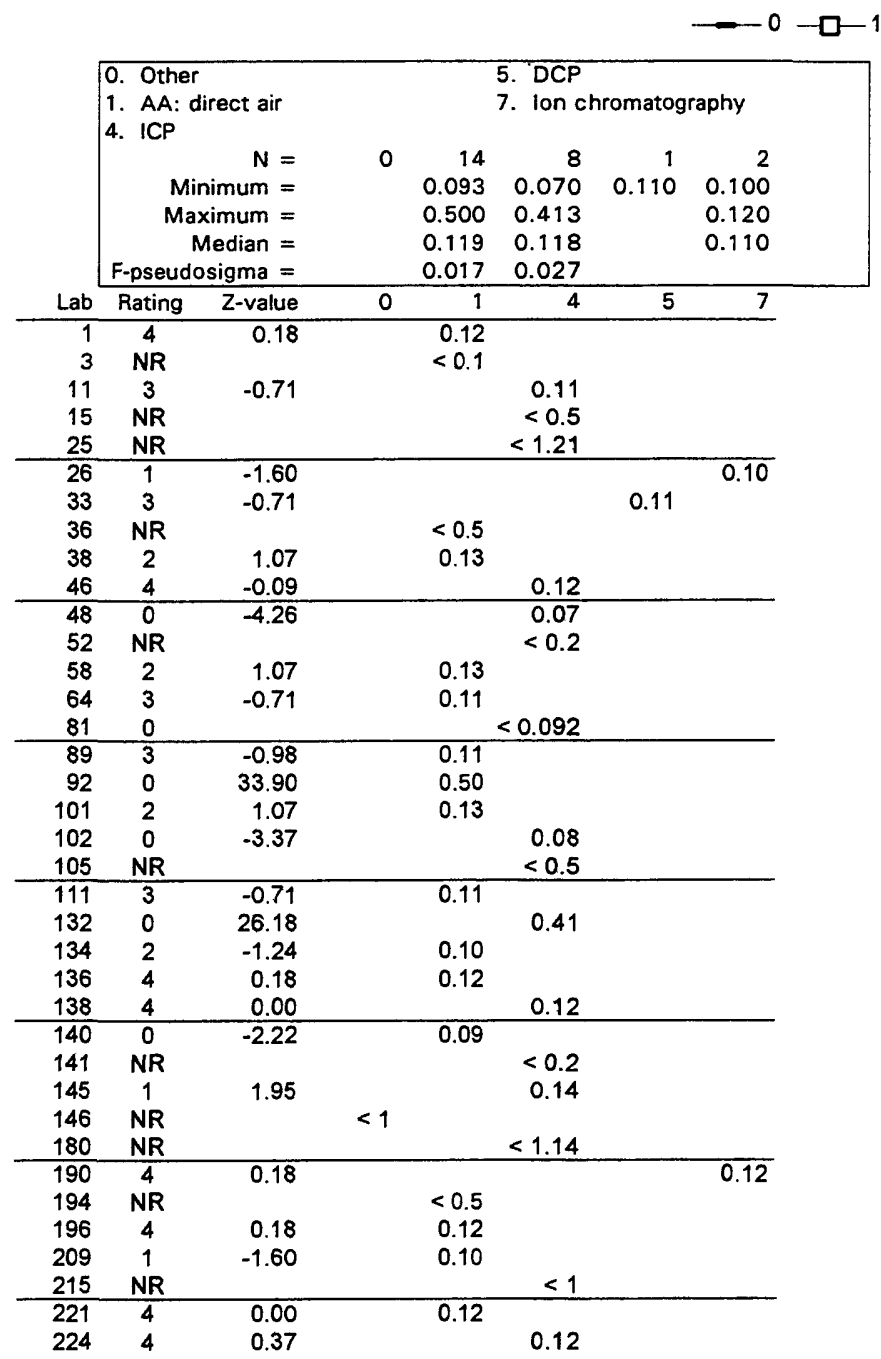


Table 16. Statistical summary of reported data for standard reference water sample P-24 (low ionic strength)--Continued Mg (Magnesium) $\mathrm{mg} / \mathrm{l}$

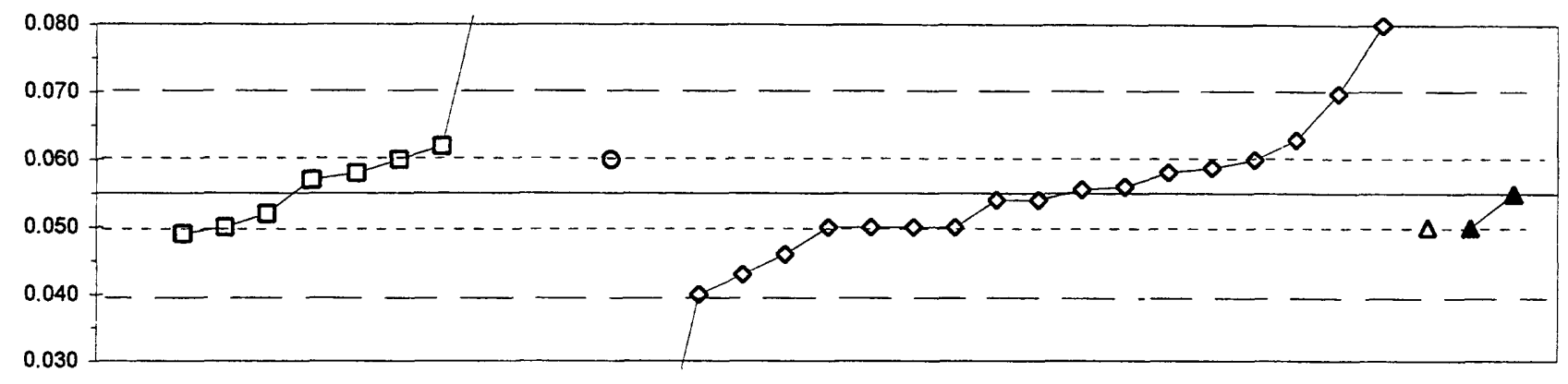

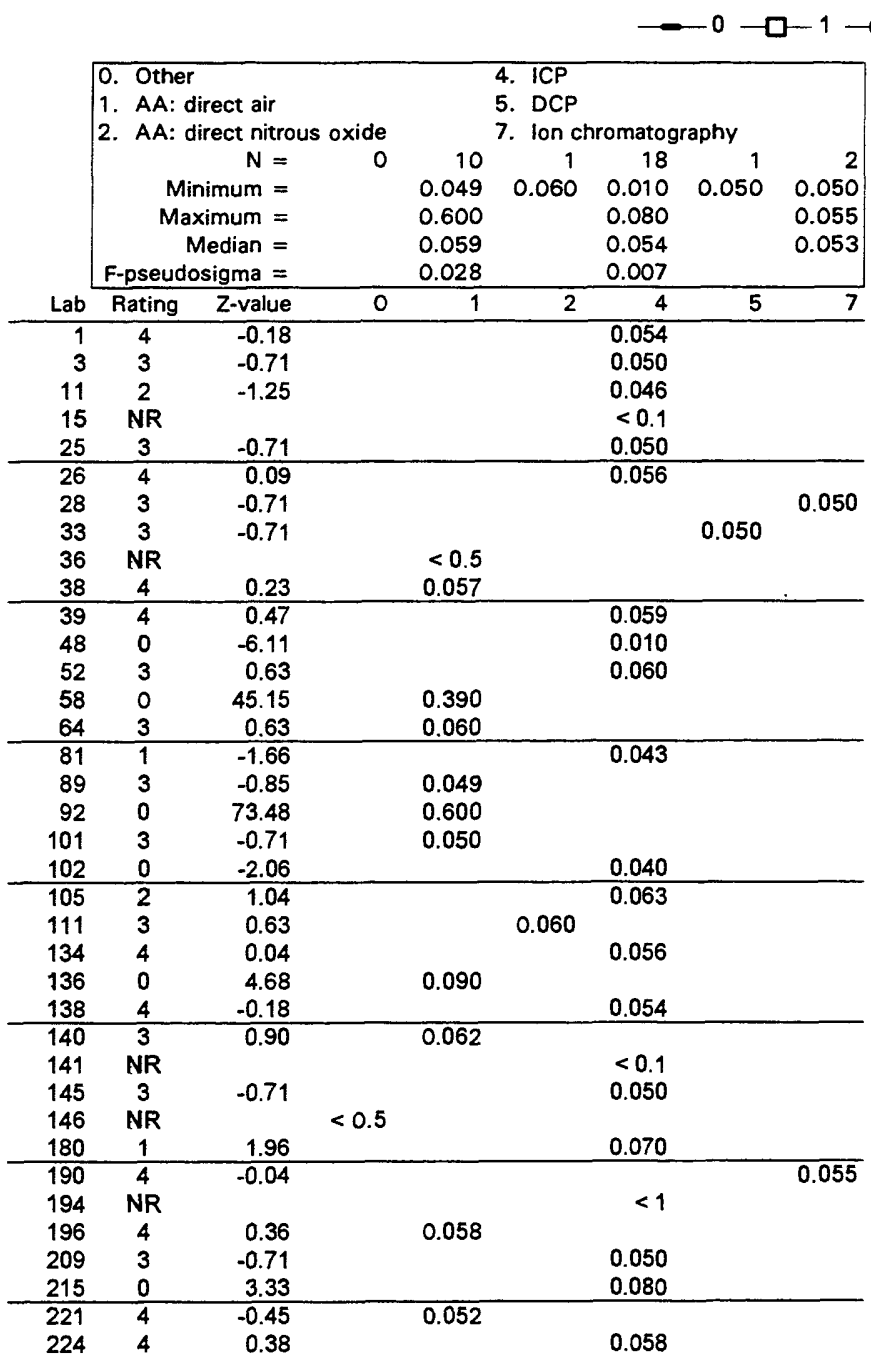


Table 16. Statistical summary of reported data for standard reference water sample P-24 (low ionic strength)--Continued $\mathrm{Na}$ (Sodium) $\mathrm{mg} / \mathrm{l}$

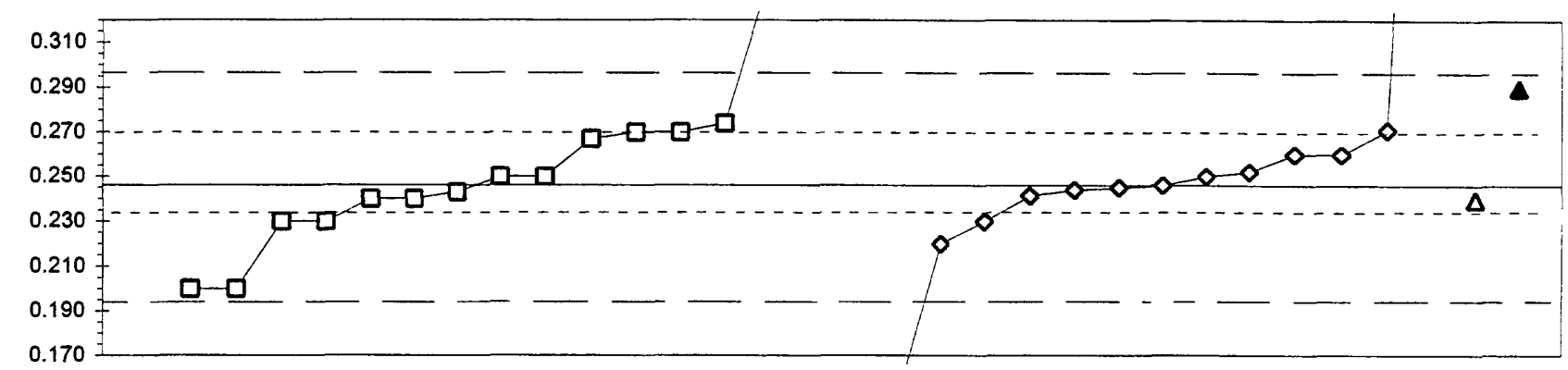

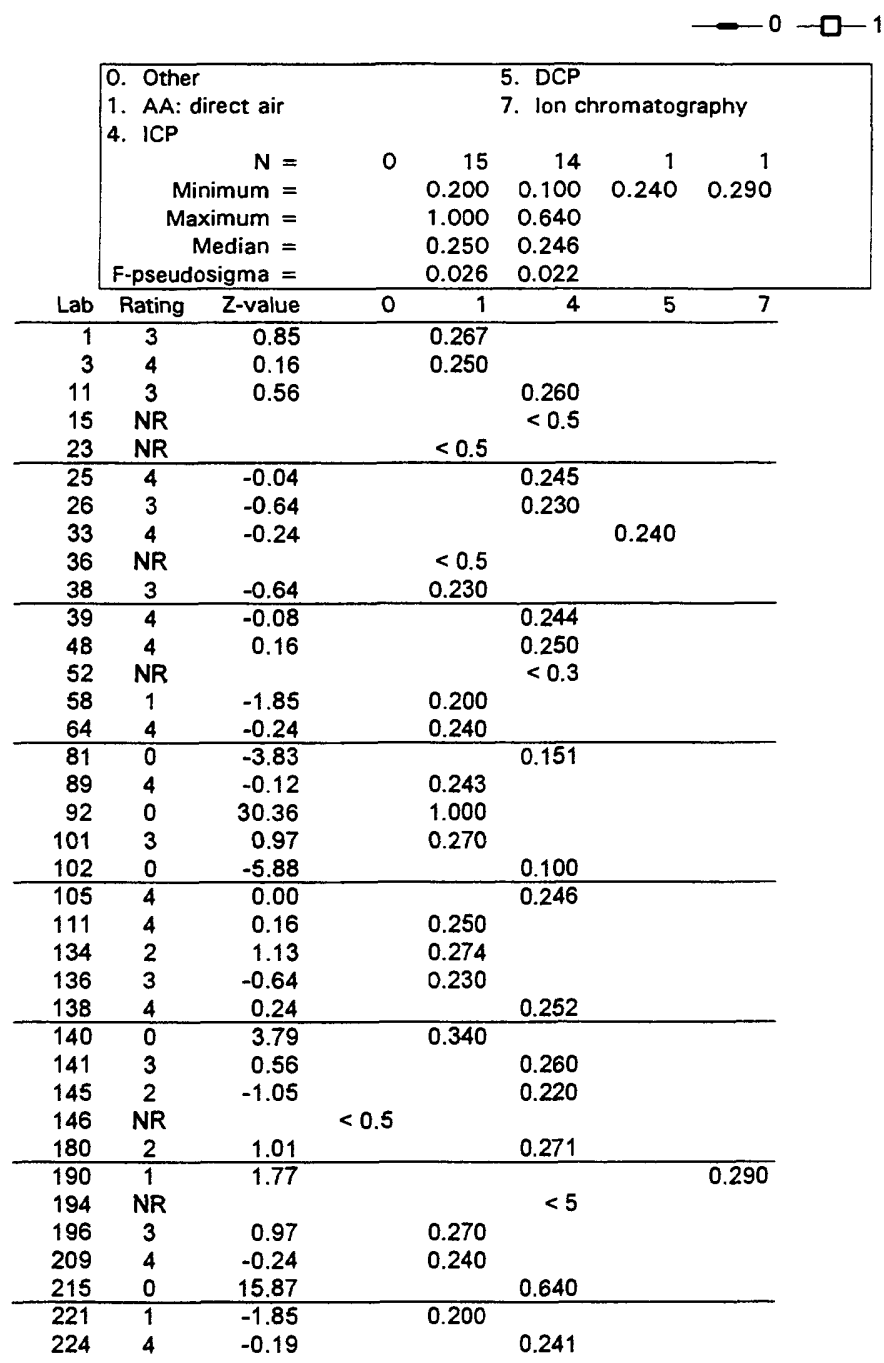


Table 16. Statistical summary of reported data for standard reference water sample P-24 (low ionic strength)--Continued pH

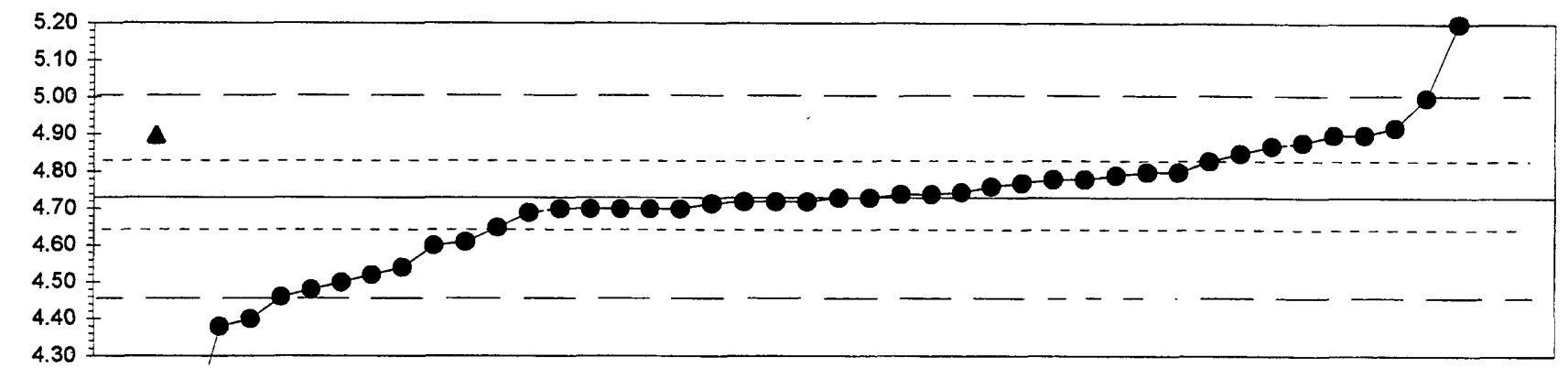

\begin{tabular}{|c|c|c|c|c|c|c|}
\hline & $\begin{array}{l}\text { 0. Other } \\
\text { 7. Ion ch } \\
\text { 41. Direc } \\
\text { F-pseudd }\end{array}$ & $\begin{array}{r}\text { romatogr } \\
\text { reading } \\
N= \\
\text { imum }= \\
\text { imum }= \\
\text { ledian }= \\
\text { sigma }=\end{array}$ & $\begin{array}{r}1 \\
3.54\end{array}$ & $4.90^{1}$ & $\begin{array}{r}44 \\
4.07 \\
7.81 \\
4.73 \\
0.11 \\
\end{array}$ & \\
\hline Lab & Rating & Z-value & 0 & 7 & 41 & \\
\hline 1 & 2 & 1.42 & & & 4.92 & \\
\hline 2 & 1 & -1.57 & & & 4.52 & \\
\hline 3 & 4 & -0.07 & & & 4.72 & \\
\hline 7 & 3 & 0.75 & & & 4.83 & \\
\hline 11 & 1 & -2.02 & & & 4.46 & \\
\hline 15 & 0 & 8.77 & & & 5.90 & \\
\hline 23 & 4 & 0.37 & & & 4.78 & \\
\hline 25 & 4 & 0.37 & & & 4.78 & \\
\hline 26 & 4 & 0.30 & & & 4.77 & \\
\hline 28 & 2 & 1.27 & & 4.90 & & \\
\hline 33 & 4 & -0.07 & & & 4.72 & \\
\hline 36 & 3 & -0.97 & & & 4.60 & \\
\hline 39 & 3 & 0.52 & & & 4.80 & \\
\hline 46 & 1 & 2.02 & & & 5.00 & \\
\hline 48 & 0 & 3.52 & & & 5.20 & \\
\hline 52 & 4 & -0.22 & & & 4.70 & \\
\hline 58 & 4 & -0.30 & & & 4.69 & \\
\hline 59 & 3 & -0.60 & & & 4.65 & \\
\hline 64 & 4 & 0.00 & & & 4.73 & \\
\hline 81 & 4 & -0.22 & & & 4.70 & \\
\hline 89 & 1 & -1.72 & & & 4.50 & \\
\hline 92 & 2 & 1.12 & & & 4.88 & \\
\hline 93 & 4 & -0.22 & & & 4.70 & \\
\hline 100 & 4 & 0.45 & & & 4.79 & \\
\hline 101 & 2 & -1.42 & & & 4.54 & \\
\hline 105 & 4 & 0.00 & & & 4.73 & \\
\hline 107 & 0 & 23.08 & & & 7.81 & \\
\hline 110 & 4 & -0.11 & & & 4.72 & \\
\hline 111 & 3 & 0.90 & & & 4.85 & \\
\hline 132 & 4 & 0.07 & & & 4.74 & \\
\hline 134 & 4 & 0.11 & & & 4.75 & \\
\hline 136 & 0 & -2.47 & & & 4.40 & \\
\hline 138 & 3 & -0.90 & & & 4.61 & \\
\hline 140 & 0 & -2.62 & & & 4.38 & \\
\hline 141 & 2 & 1.27 & & & 4.90 & \\
\hline 143 & 4 & -0.07 & & & 4.72 & \\
\hline 145 & 4 & -0.22 & & & 4.70 & \\
\hline 146 & 0 & -8.92 & 3.54 & & & \\
\hline 180 & 4 & -0.22 & & & 4.70 & \\
\hline 190 & 1 & -1.87 & & & 4.48 & \\
\hline 194 & 2 & 1.05 & & & 4.87 & \\
\hline 203 & 4 & 0.22 & & & 4.76 & \\
\hline 209 & 4 & 0.07 & & & 4.74 & \\
\hline 215 & 2 & 1.27 & & & 4.90 & \\
\hline 221 & 3 & 0.52 & & & 4.80 & \\
\hline 224 & 0 & -4.95 & & & 4.07 & \\
\hline
\end{tabular}


Table 16. Statistical summary of reported data for standard reference water sample P-24 (low ionic strength)--Continued PO4 as P (Orthoposphate) $\mathrm{mg} / \mathrm{l}$
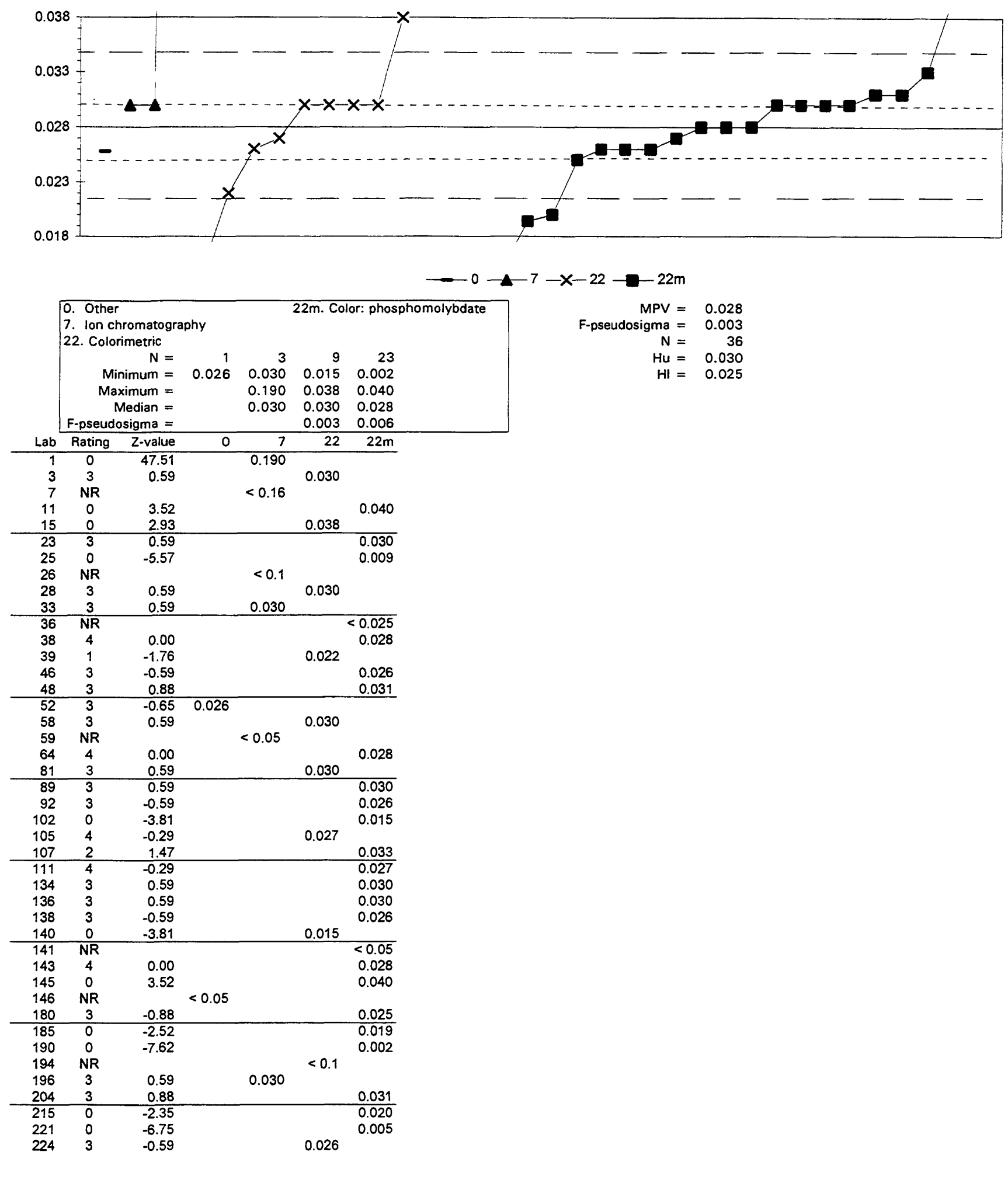
Table 16. Statistical summary of reported data for standard reference water sample P-24 (low ionic strength)--Continued SO4 (Sulfate) $\mathrm{mg} / \mathrm{l}$

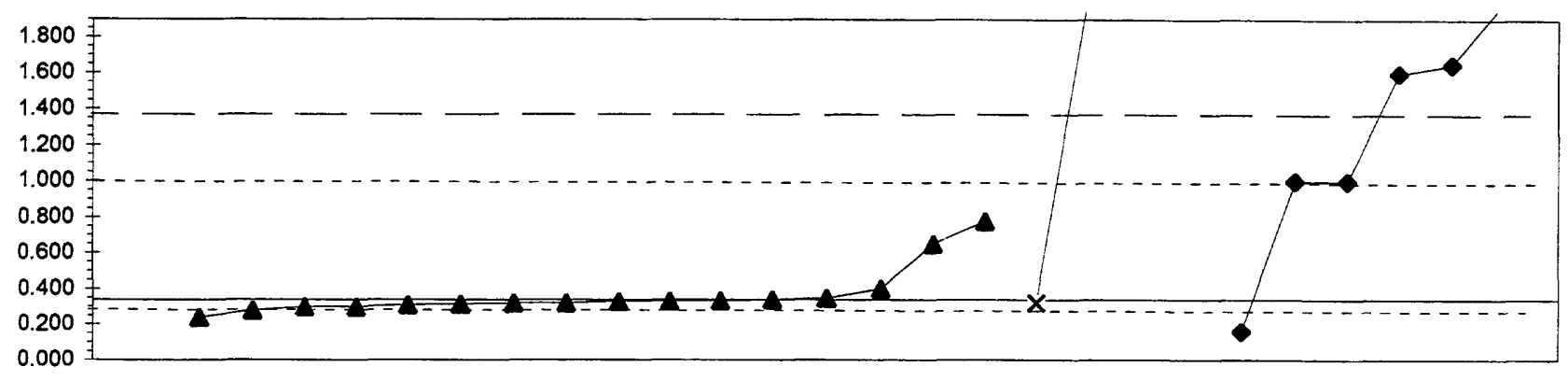

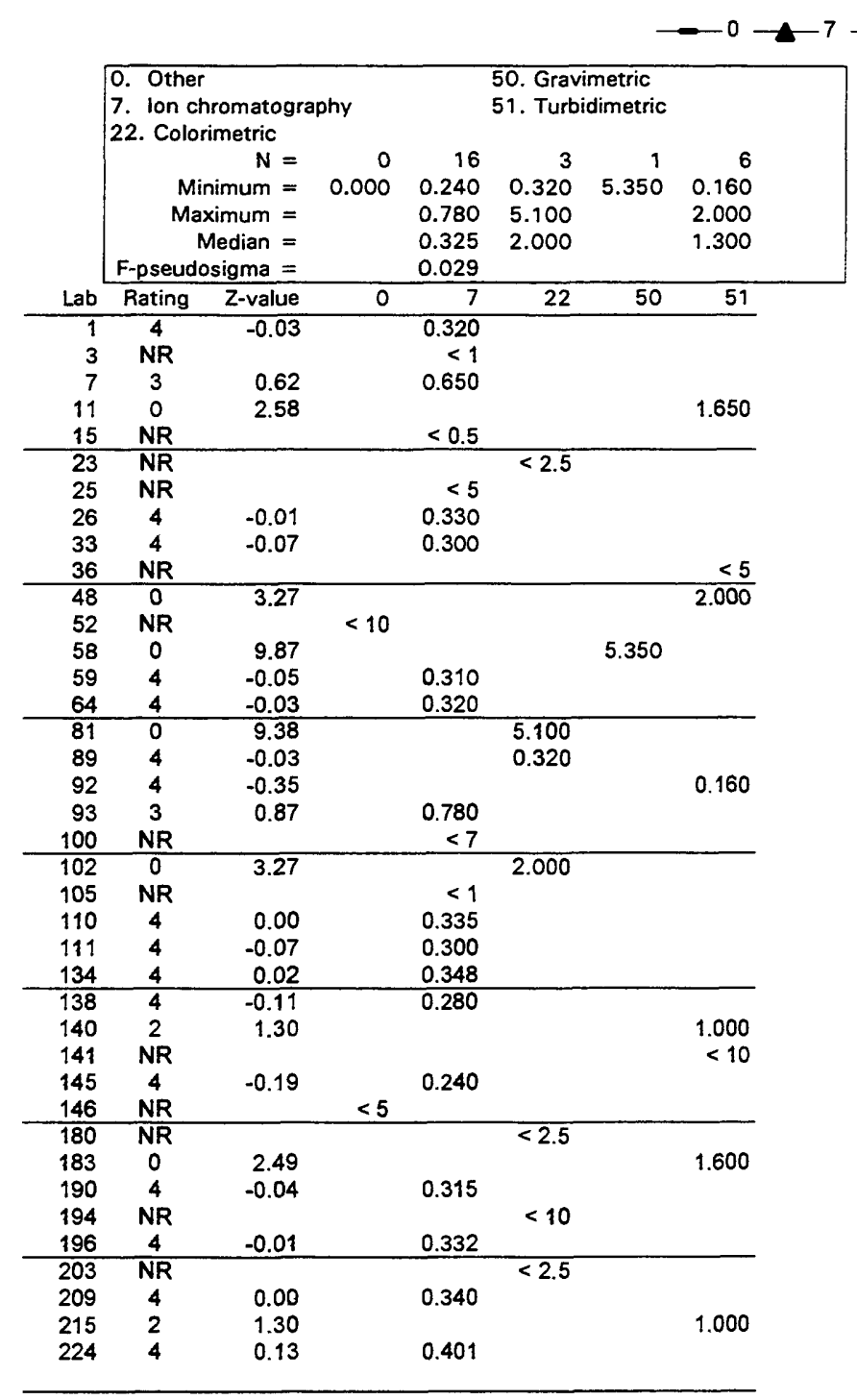


Table 16. Statistical summary of reported data for standard reference water sample P-24 (low ionic strength)--Continued Specific Conductance $\mu \mathrm{S} / \mathrm{cm}$

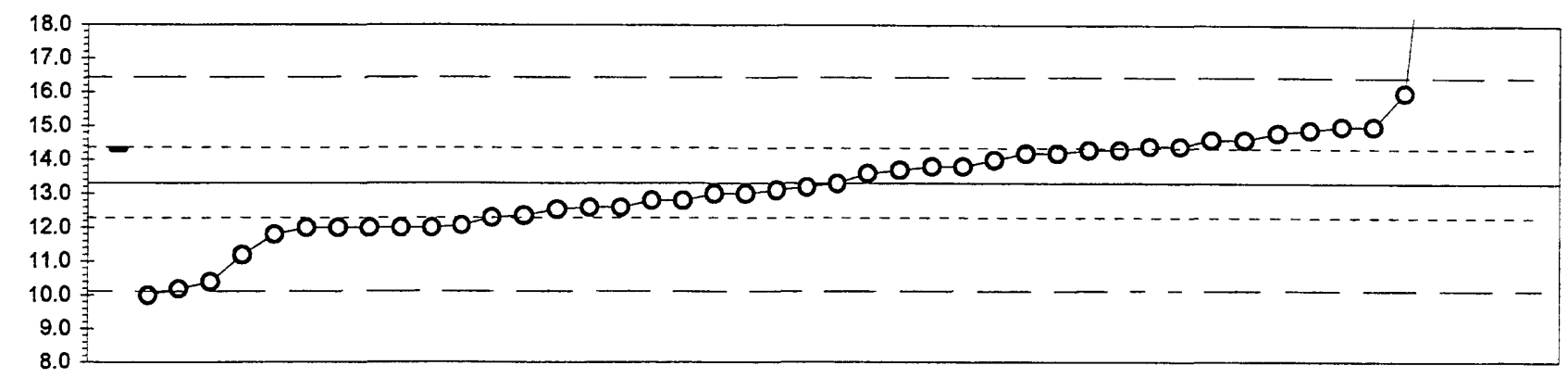

\begin{tabular}{|c|c|c|c|c|}
\hline \multirow[b]{2}{*}{ Lab } & $\begin{array}{l}\text { 0. Othe } \\
\text { 41. Direc }\end{array}$ & $\begin{array}{r}\text { reading } \\
\mathrm{N}= \\
\text { imum }= \\
\text { imum }= \\
\text { iedian }= \\
\text { sigma }=\end{array}$ & $\begin{array}{r}1 \\
14.3 \\
\end{array}$ & $\begin{array}{r}44 \\
10.0 \\
660.0 \\
13.3 \\
1.6 \\
\end{array}$ \\
\hline & Rating & $Z$-value & 0 & 41 \\
\hline 1 & 3 & -0.79 & & 12.1 \\
\hline 2 & 3 & -0.60 & & 12.4 \\
\hline 3 & 3 & 0.84 & & 14.6 \\
\hline 7 & 4 & 0.32 & & 13.8 \\
\hline 11 & 4 & -0.32 & & 12.8 \\
\hline 15 & 3 & 0.96 & & 14.8 \\
\hline 23 & 4 & -0.45 & & 12.6 \\
\hline 25 & 1 & 1.73 & & 16.0 \\
\hline 26 & 4 & 0.32 & & 13.8 \\
\hline 27 & 3 & 0.64 & & 14.3 \\
\hline 28 & 0 & 415.43 & & 660.0 \\
\hline 33 & 3 & 0.71 & & 14.4 \\
\hline 36 & 3 & -0.96 & & 11.8 \\
\hline 38 & 4 & 0.00 & & 13.3 \\
\hline 39 & 1 & -1.86 & & 10.4 \\
\hline 46 & 3 & 0.64 & & 14.3 \\
\hline 48 & 4 & $\begin{array}{r}-0.49 \\
\end{array}$ & & 12.5 \\
\hline 52 & 3 & -0.84 & & 12.0 \\
\hline 58 & 4 & 0.26 & & 13.7 \\
\hline 59 & 4 & -0.45 & & 12.6 \\
\hline 64 & 4 & -0.13 & & $\overline{13.1}$ \\
\hline 81 & 3 & -0.84 & & 12.0 \\
\hline 89 & 2 & -1.35 & & 11.2 \\
\hline 93 & 4 & -0.19 & & 13.0 \\
\hline 100 & 3 & -0.84 & & 12.0 \\
\hline 101 & 4 & -0.19 & & 13.0 \\
\hline 105 & 4 & -0.32 & & 12.8 \\
\hline 107 & 2 & 1.03 & & 14.9 \\
\hline 110 & 2 & 1.09 & & 15.0 \\
\hline 111 & 4 & 0.19 & & 13.6 \\
\hline 134 & 3 & 0.58 & & 14.2 \\
\hline 136 & 3 & 0.71 & & 14.4 \\
\hline 140 & 3 & 0.84 & & 14.6 \\
\hline 141 & 4 & -0.06 & & 13.2 \\
\hline 143 & 3 & -0.84 & & 12.0 \\
\hline 145 & 4 & 0.45 & & 14.0 \\
\hline 146 & 3 & 0.64 & 14.3 & \\
\hline 180 & 0 & -2.12 & & 10.0 \\
\hline 183 & 3 & -0.84 & & 12.0 \\
\hline 190 & 3 & 0.58 & & 14.2 \\
\hline 194 & 3 & -0.64 & & 12.3 \\
\hline 196 & 0 & 17.73 & & 40.9 \\
\hline 203 & 1 & -1.99 & & 10.2 \\
\hline 215 & 0 & 7.13 & & 24.4 \\
\hline 224 & 2 & 1.09 & & 15.0 \\
\hline
\end{tabular}


Table 17. Statistical summary of reported data for standard reference water sample Hg-2O (mercury)

Definition of analytical methods, abbreviations, and symbols

Analvtical methods

0. Other/Not reported

8. AA: cold vapor = atomic absorption: cold vapor

Abbreviations and symbols

$\mathbf{N}=\quad$ number of samples

St dev = $\quad$ traditional standard deviation

MPV $=\quad$ most probable vaiue

F-pseudosigma $=\quad$ nonparametric statistic deviation

$\mathrm{Hu}=\quad$ upper hinge value

$\mathrm{HI}=\quad$ lower hinge value

$\mu \mathrm{g} / \mathrm{L}=\quad$ micrograms per liter

$\mathrm{Lab}=\quad$ laboratory code number

NR $=\quad$ not rated, less than value reported

$<=\quad$ less than 
Table 17. Statistical summary of reported data for standard reference water sample Hg-20 (mercury)--Continued Hg (Mercury) $\mu \mathrm{g} / \mathrm{l}$

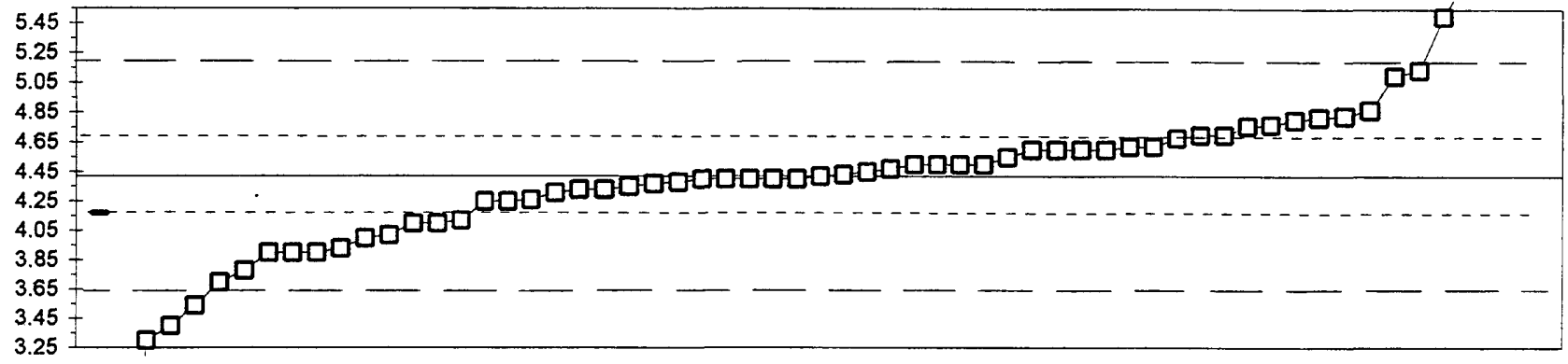

$0-\square-8$

\begin{tabular}{|c|c|c|c|c|c|c|c|c|c|}
\hline \multirow[b]{2}{*}{ Lab } & $\begin{array}{l}\text { 0. Othe } \\
\text { 8. AA: } c \\
\text { 0. Othe } \\
\text { F-pseud }\end{array}$ & $\begin{aligned} \text { Id vapor } & \\
\mathrm{N} & = \\
\text { imum } & = \\
\text { imum } & = \\
\text { ledian } & = \\
\text { sigma } & =\end{aligned}$ & $\begin{array}{r}1 \\
4.17\end{array}$ & $\begin{array}{r}60 \\
1.00 \\
6.35 \\
4.43 \\
0.37 \\
\end{array}$ & \multirow[t]{2}{*}{$\mathrm{F}$} & \multicolumn{2}{|c|}{$\begin{aligned} M P V & = \\
\text { F-pseudosigma } & = \\
N & = \\
H u & = \\
H I & =\end{aligned}$} & \multicolumn{2}{|l|}{$\begin{array}{r}4.42 \\
0.38 \\
61 \\
4.68 \\
4.17\end{array}$} \\
\hline & Rating & $Z$-value & 0 & 8 & & Rating & $Z$-value & 0 & 8 \\
\hline 1 & 3 & 0.53 & & 4.62 & 194 & 4 & 0.21 & & 4.50 \\
\hline 3 & 4 & -0.24 & & 4.33 & 198 & 1 & -1.90 & & 3.70 \\
\hline 11 & 4 & -0.05 & & 4.40 & 203 & 4 & -0.45 & & 4.25 \\
\hline 13 & 3 & 0.69 & & 4.68 & 213 & 4 & 0.48 & & 4.60 \\
\hline 15 & 4 & -0.05 & & 4.40 & 215 & 0 & 4.18 & & 6.00 \\
\hline 16 & 2 & -1.11 & & 4.00 & 219 & 2 & -1.38 & & 3.90 \\
\hline 18 & 4 & -0.05 & & 4.40 & 221 & 3 & -0.85 & & 4.10 \\
\hline 24 & 1 & 1.80 & & 5.10 & 231 & 4 & -0.05 & & 4.40 \\
\hline 32 & 3 & -0.66 & 4.17 & & 234 & 4 & 0.03 & & 4.43 \\
\hline 34 & 4 & 0.21 & & 4.50 & 235 & 0 & 4.18 & & 6.00 \\
\hline 36 & 1 & -1.69 & & 3.78 & 241 & 4 & -0.42 & & 4.26 \\
\hline 39 & 3 & -0.85 & & 4.10 & & & & & \\
\hline 42 & 2 & -1.30 & & 3.93 & & & & & \\
\hline 45 & 1 & 1.90 & & 5.14 & & & & & \\
\hline 46 & 4 & -0.29 & & 4.31 & & & & & \\
\hline
\end{tabular}


Table 18. Statistical summary of reported data for standard reference sediment sample SED-5 (bed material)

Definition of analytical methods, abbreviations, and symbols

Analytical methods

0. Other/Not reported

1. AA: direct, air

$=$ atomic absorption: direct,air

2. AA: direct, $\mathrm{N} 2 \mathrm{O}$

3. AA: graphite fumace

= atomic absorption: direct, nitrous oxide

4. ICP

5. DCP

6. ICPMS

10. AA: extraction

11. AA: hydride

12. Flame emission

22. Color:

= atomic absorption: graphite furnace

$=$ inductively coupled plasma

$=$ direct current plasma

$=\quad$ inductively coupled plasma/mass spectrometry

$=$ atomic absorption: extraction [chelating agent(s) specified]

$=$ atomic absorption: hydride [reducing agent specified]

= flame emission

$=$ colorimetric [color reagent specified]

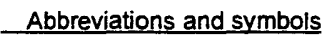

$\begin{array}{rlr}\mathrm{N} & = & \text { number of samples } \\ \text { St dev } & = & \text { traditional standard deviation } \\ \mathrm{MPV} & = & \text { most probable value } \\ \text { F-pseudosigma } & = & \text { nonparametric statistic deviation } \\ \mathrm{Hu} & = & \text { upper hinge value } \\ \mathrm{HI} & = & \text { lower hinge value } \\ \mu \mathrm{g} / \mathrm{g} & = & \text { micrograms per liter } \\ \mathrm{mg} / \mathrm{g} & = & \text { milligrams per liter } \\ \mathrm{Lab} & = & \text { laboratory code number } \\ \mathrm{NR} & = & \text { not rated, less than value reported } \\ < & = & \text { less than }\end{array}$

\begin{tabular}{|c|c|c|c|c|c|}
\hline \multicolumn{2}{|c|}{ Constituent } & \multirow{2}{*}{$\frac{\text { page }}{109}$} & \multicolumn{2}{|c|}{ Constituent } & \multirow{2}{*}{$\frac{\text { page }}{122}$} \\
\hline $\mathrm{Ag}$ & Silver & & $\mathrm{Li}$ & Lithium & \\
\hline $\mathrm{Al}$ & Aluminium & 110 & $\mathrm{Mg}$ & Magnesium & 123 \\
\hline As & Arsenic & 111 & $\mathrm{Mn}$ & Manganese & 124 \\
\hline B & Boron & 112 & Mo & Molybdenum & 125 \\
\hline $\mathrm{Ba}$ & Barium & 113 & $\mathrm{Na}$ & Sodium & 126 \\
\hline $\mathrm{Be}$ & Beryllium & 114 & $\mathrm{Ni}$ & Nickel & 127 \\
\hline $\mathrm{Ca}$ & Calcium & 115 & $\mathrm{~Pb}$ & Lead & 128 \\
\hline $\mathrm{Cd}$ & Cadmium & 116 & Sb & Antimony & 129 \\
\hline Co & Cobalt & 117 & Se & Selenium & 130 \\
\hline $\mathrm{Cr}$ & Chromium & 118 & $\mathrm{SiO} 2$ & Silica & 131 \\
\hline $\mathrm{Cu}$ & Copper & 119 & $\mathrm{Sr}$ & Strontium & 132 \\
\hline $\mathrm{Fe}$ & Iron & 120 & $v$ & Vanadium & 133 \\
\hline K & Potassium & 121 & $\mathrm{Zn}$ & Zinc & 134 \\
\hline
\end{tabular}


Table 18. Statistical summary of reported data for standard reference sediment sample SED-5 (bed material)--Continued Ag (Silver)

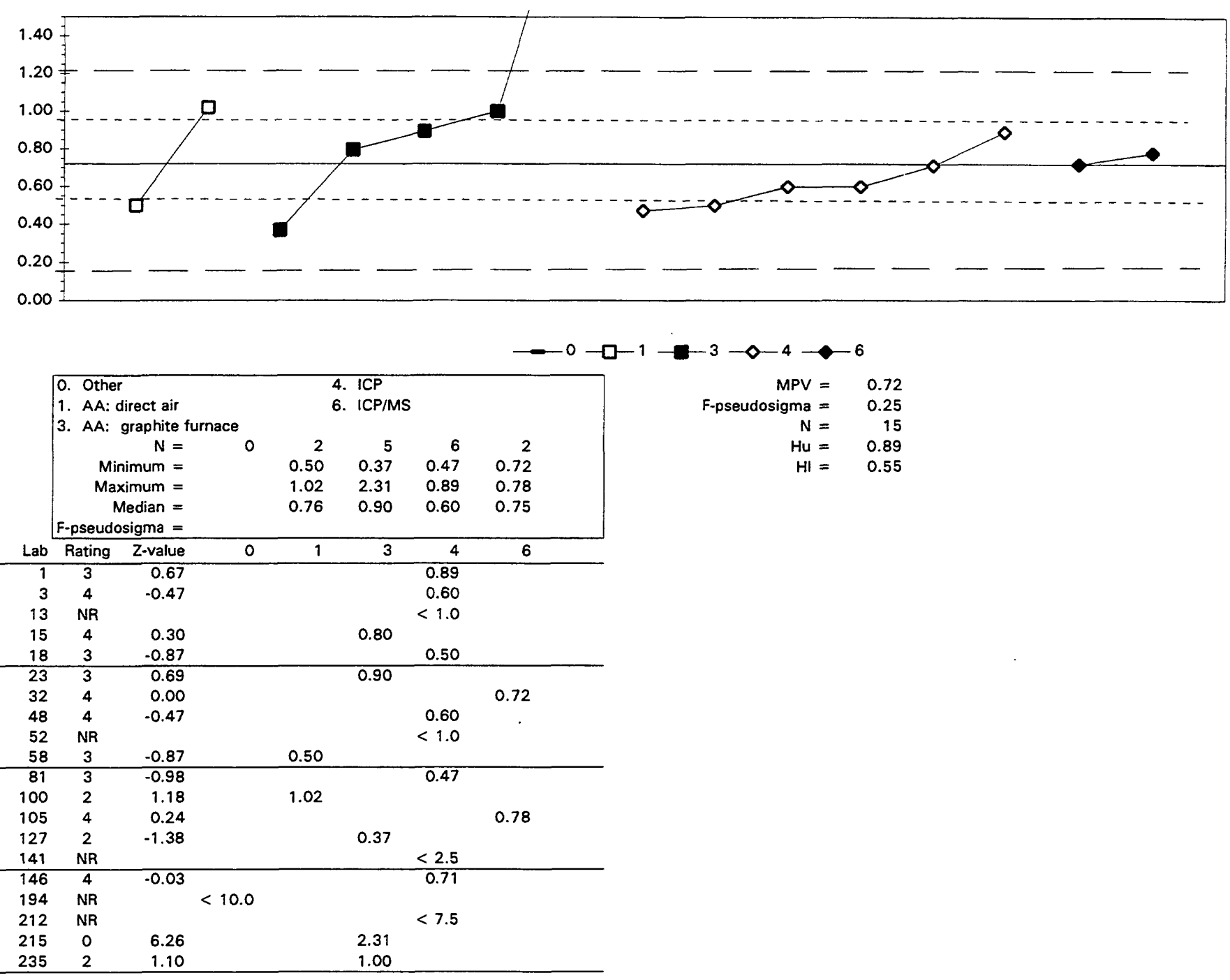


Table 18. Statistical summary of reported data for standard reference sediment sample SED-5 (bed material)--Continued Al (Aluminum) $\mu \mathrm{g} / \mathrm{g}$

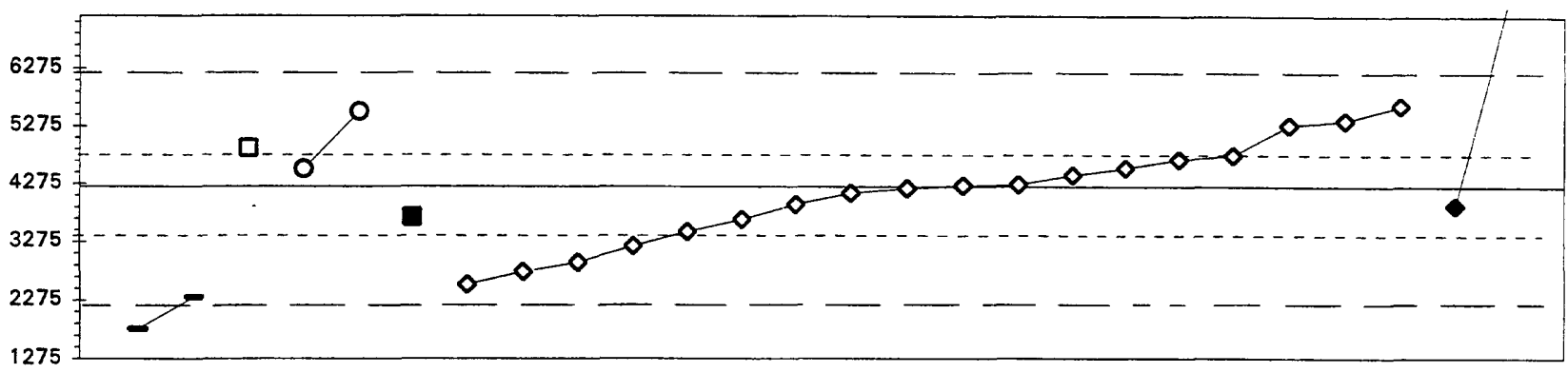

$$
-0-0-1-0-2 \rightarrow-3-0-4 \multimap 6
$$

\begin{tabular}{|c|c|c|c|c|c|c|c|c|}
\hline & \multirow{2}{*}{\multicolumn{3}{|c|}{$\begin{array}{l}\text { O. Other } \\
\text { 1. AA: direct air } \\
\text { 2. AA: direct nitrous oxide }\end{array}$}} & \multicolumn{4}{|c|}{$\begin{array}{l}\text { 3. AA: graphite furnace } \\
\text { 4. ICP } \\
\text { 6. ICP/MS }\end{array}$} & \multirow{6}{*}{$\begin{array}{r}2 \\
3900 \\
7590 \\
5745\end{array}$} \\
\hline & 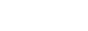 & & & 1 & & 1 & 18 & \\
\hline & Mir & mum $=$ & 1790 & 4900 & 4540 & 3710 & 2540 & \\
\hline & Mas & mum = & 2330 & 4900 & 5540 & 3710 & 5640 & \\
\hline & $=-$ sseudo & $\begin{array}{l}\text { edian }= \\
\text { iama }=\end{array}$ & 2060 & 4900 & 5040 & 3710 & 4227 & \\
\hline & \multicolumn{2}{|c|}{ F-pseudosigma $=$} & & & & & & \\
\hline Lab & Rating & Z-value & 0 & 1 & 2 & 3 & 4 & 6 \\
\hline 1 & 3 & 0.56 & & & & & 4771 & \\
\hline 3 & 2 & -1.50 & & & & & 2760 & \\
\hline 13 & 2 & 1.10 & & & & & 5300 & \\
\hline 15 & 4 & 0.21 & & & & & 4430 & \\
\hline 18 & 2 & -1.33 & & & & & 2920 & \\
\hline 23 & 2 & 1.34 & & & 5540 & & & \\
\hline 28 & 0 & -2.49 & 1790 & & & & & \\
\hline 32 & 0 & 3.43 & & & & & & 7590 \\
\hline 48 & 4 & 0.33 & & & & & 4552 & \\
\hline 52 & 2 & -1.05 & & & & & 3200 & \\
\hline 58 & 3 & 0.69 & & 4900 & & & & \\
\hline 68 & 4 & 0.02 & & & & & 4250 & \\
\hline 81 & 3 & -0.53 & & & & 3710 & & \\
\hline 100 & 4 & -0.11 & & & & & 4118 & \\
\hline 105 & 4 & -0.33 & & & & & & 3900 \\
\hline 121 & 2 & 1.18 & & & & & 5380 & \\
\hline 127 & 4 & -0.31 & & & & & 3920 & \\
\hline 132 & 4 & -0.02 & & & & & 4204 & \\
\hline 141 & 3 & -0.79 & & & & & 3450 & \\
\hline 146 & 4 & 0.04 & & & & & 4270 & \\
\hline 154 & 4 & 0.48 & & & & & 4700 & \\
\hline 194 & 1 & -1.94 & 2330 & & & & & \\
\hline 210 & 2 & 1.44 & & & & & 5640 & \\
\hline 212 & 1 & -1.72 & & & & & 2540 & \\
\hline 215 & 3 & -0.58 & & & & & 3660 & \\
\hline 235 & 4 & 0.32 & & & 4540 & & & \\
\hline
\end{tabular}


Table 18. Statistical summary of reported data for standard reference sediment sample SED-5 (bed material)--Continued As (Arsenic) $\mu \mathrm{g} / \mathrm{g}$

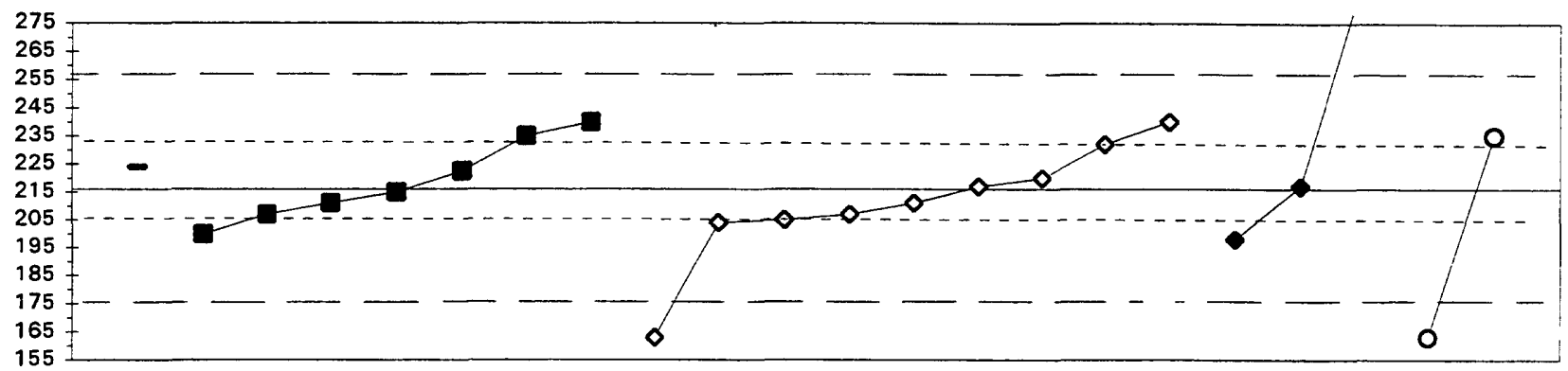

$-0 \rightarrow-3-0-4-6-0-11$

\begin{tabular}{|c|c|c|c|c|c|c|c|}
\hline & \multicolumn{3}{|c|}{$\begin{array}{l}\text { O. Other } \\
\text { 3. AA: graphite furnace } \\
\text { 4. ICP }\end{array}$} & \multicolumn{3}{|c|}{$\begin{array}{l}\text { 6. ICP/MS } \\
\text { 11. AA: hydride }\end{array}$} & \multirow[b]{2}{*}{$\begin{array}{r}2 \\
163 \\
235 \\
199\end{array}$} \\
\hline & $\begin{array}{r}\text { Min } \\
\text { Max } \\
M \\
\text { F-pseudos }\end{array}$ & $\begin{array}{r}N= \\
\text { imum }= \\
\text { imum }= \\
\text { edian }= \\
\text { igma }=\end{array}$ & $\begin{array}{r}1 \\
224\end{array}$ & $\begin{array}{r}7 \\
200 \\
240 \\
215 \\
15 \\
\end{array}$ & $\begin{array}{r}9 \\
163 \\
240 \\
211 \\
11 \\
\end{array}$ & $\begin{array}{r}3 \\
198 \\
294 \\
217\end{array}$ & \\
\hline Lab & Rating & $Z$-value & 0 & 3 & 4 & 6 & 11 \\
\hline 1 & 3 & 0.95 & & & & & 235 \\
\hline 3 & 3 & -0.55 & & & 205 & & \\
\hline 13 & 3 & -0.80 & & 200 & & & \\
\hline 15 & 3 & 0.80 & & & 232 & & \\
\hline 18 & 4 & -0.45 & & 207 & & & \\
\hline 30 & 4 & 0.05 & & & & 217 & \\
\hline 32 & 0 & 3.90 & & & & 294 & \\
\hline 48 & 4 & 0.32 & & 223 & & & \\
\hline 52 & 4 & 0.20 & & & 220 & & \\
\hline 58 & 0 & -2.65 & & & & & 163 \\
\hline 81 & 4 & -0.25 & & 211 & & & \\
\hline 100 & 3 & 0.95 & & 235 & & & \\
\hline 105 & 3 & -0.90 & & & & 198 & \\
\hline 127 & 2 & 1.20 & & 240 & & & \\
\hline 141 & 3 & -0.60 & & & 204 & & \\
\hline 146 & 4 & 0.05 & & & 217 & & \\
\hline 154 & 2 & 1.20 & & & 240 & & \\
\hline 194 & 4 & 0.40 & 224 & & & & \\
\hline 210 & 0 & -2.65 & & & 163 & & \\
\hline 212 & 4 & -0.25 & & & 211 & & \\
\hline 215 & 4 & -0.45 & & & 207 & & \\
\hline 235 & 4 & -0.05 & & 215 & & & \\
\hline
\end{tabular}


Table 18. Statistical summary of reported data for standard reference sediment sample SED-5 (bed material)--Continued B (Boron)

$\mu \mathrm{g} / \mathrm{g}$

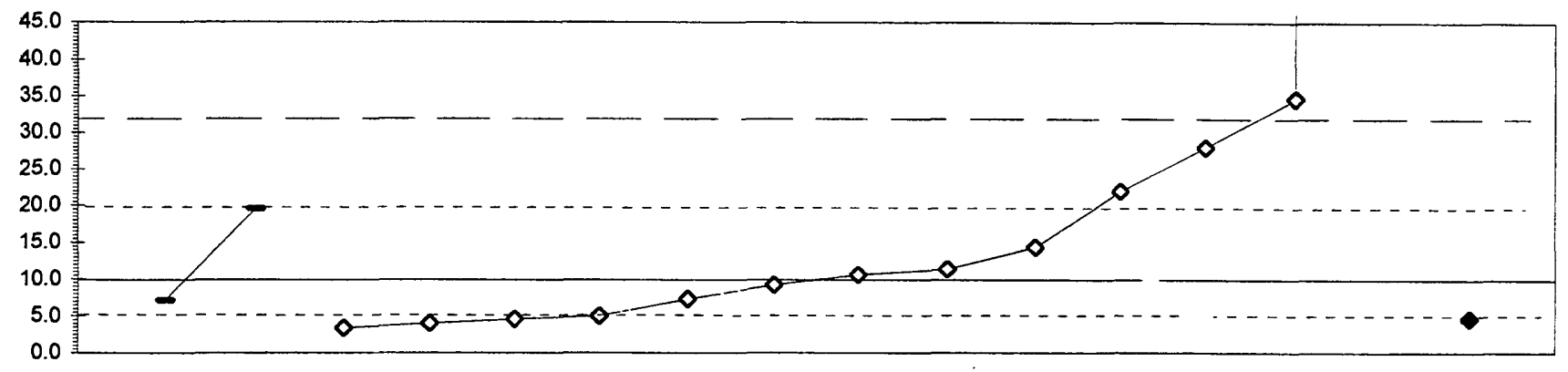

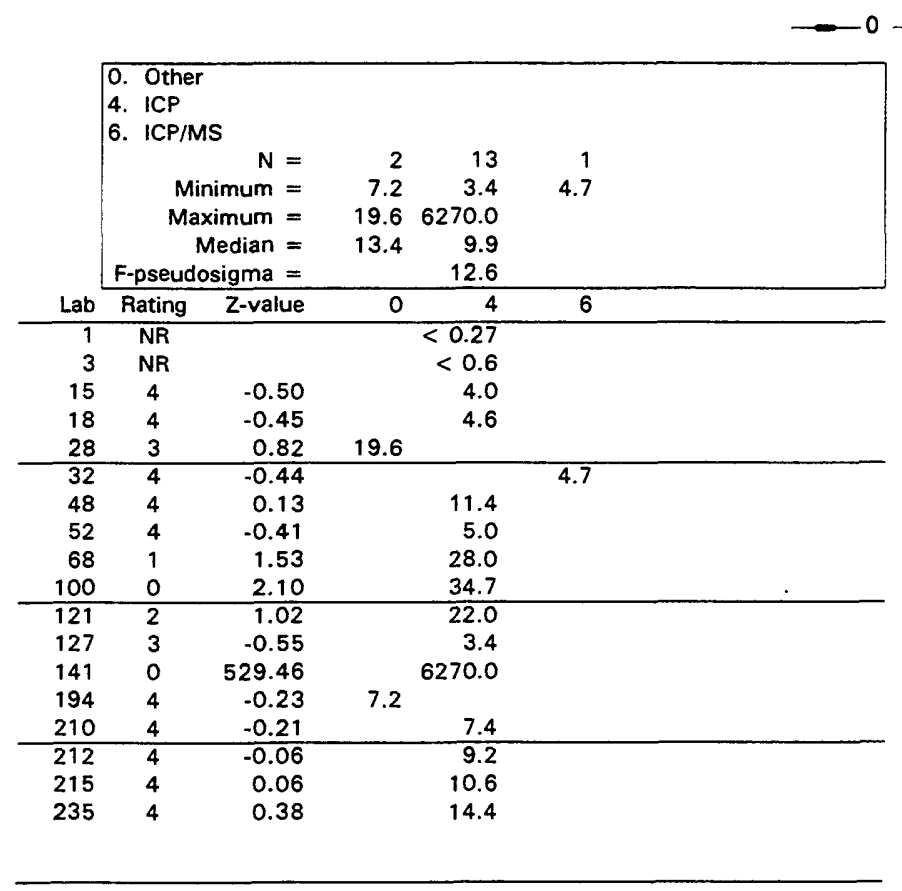


Table 18. Statistical summary of reported data for standard reference sediment sample SED-5 (bed material)--Continued Ba (Barium) $\mu \mathrm{g} / \mathrm{g}$

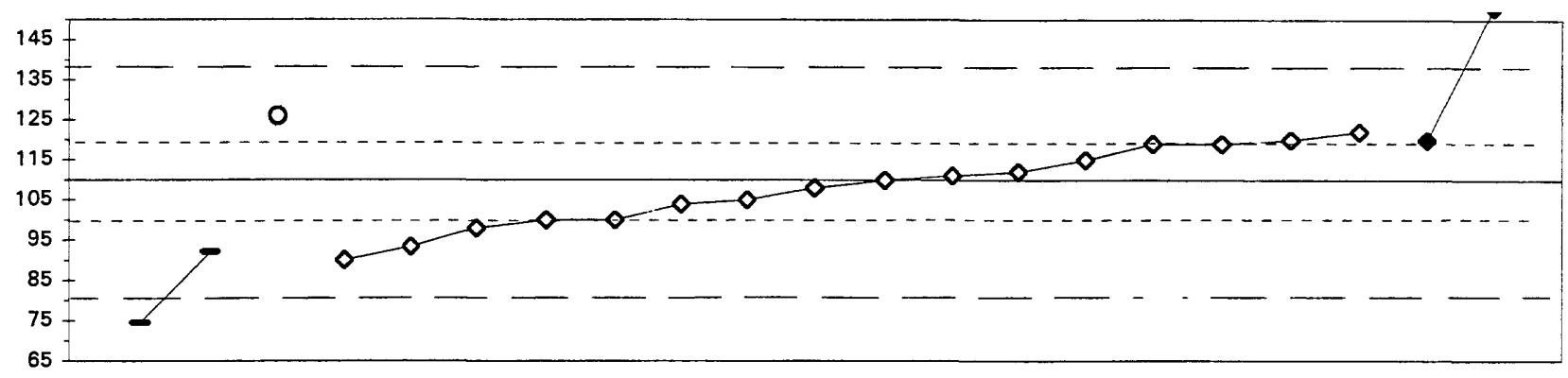

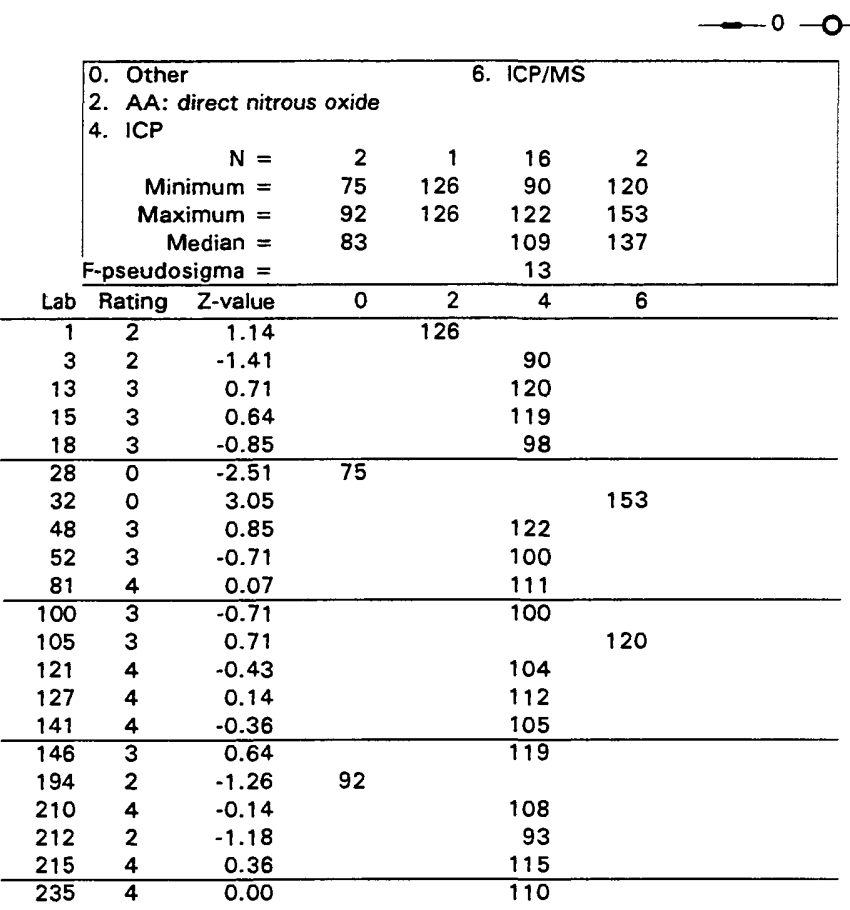


Table 18. Statistical summary of reported data for standard reference sediment sample SED-5 (bed material)--Continued $\mathrm{Be}$ (Beryllium)

$\mu \mathrm{g} / \mathrm{g}$

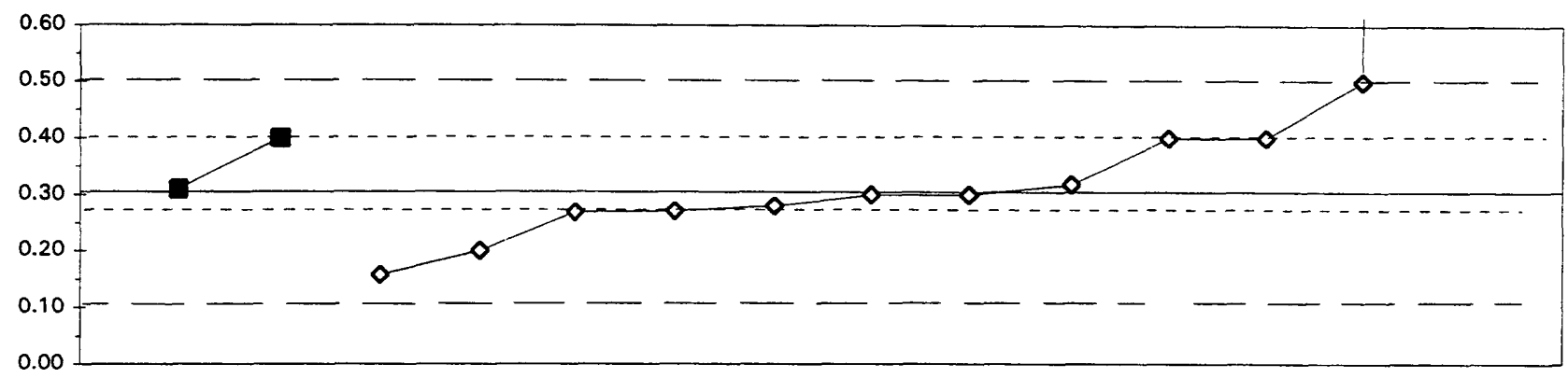

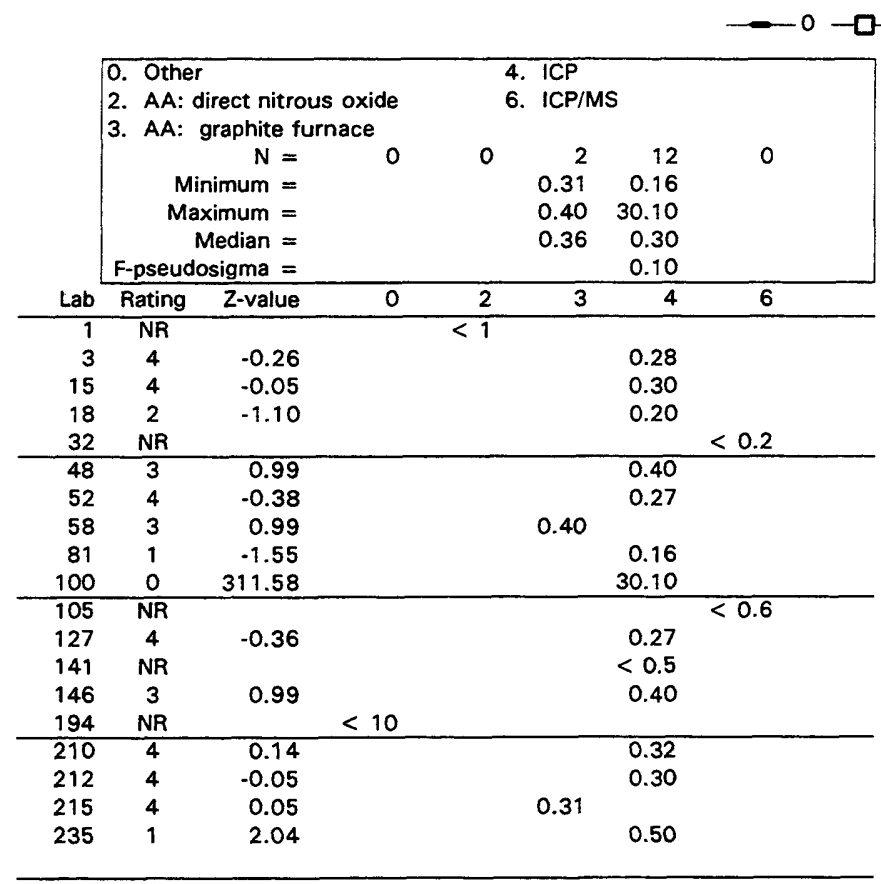


Table 18. Statistical summary of reported data for standard reference sediment sample SED-5 (bed material)--Continued Ca (Calcium) $\mathrm{mg} / \mathrm{g}$

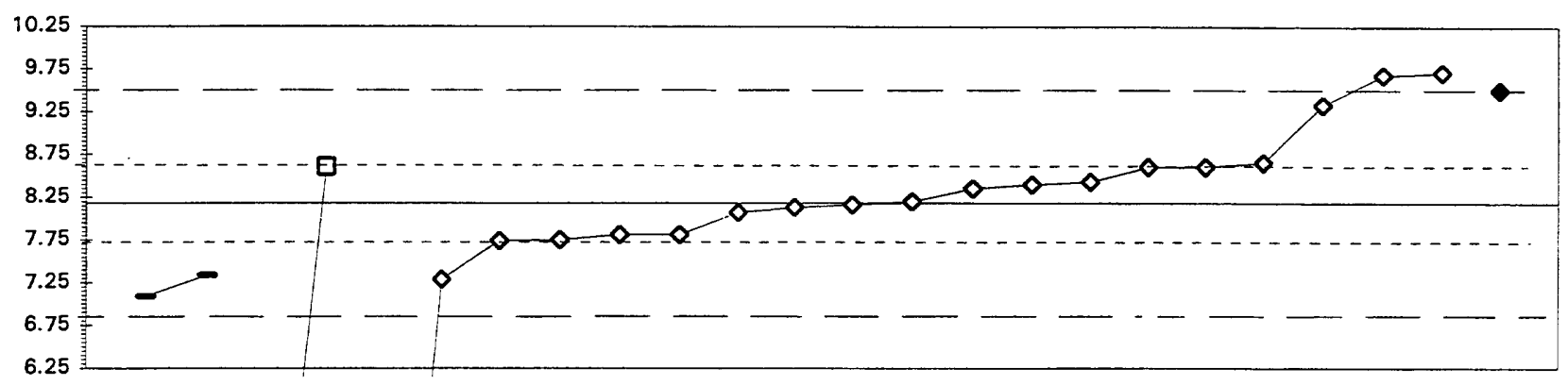

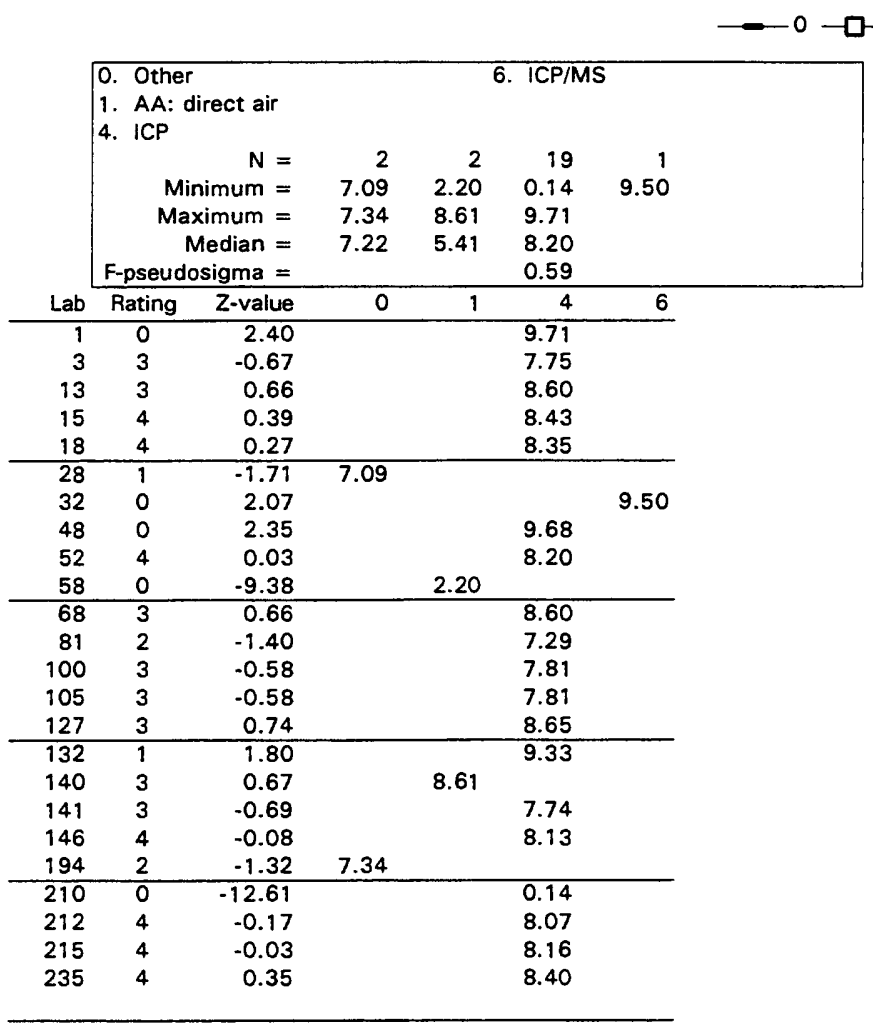


Table 18. Statistical summary of reported data for standard reference sediment sample SED-5 (bed material)-.Continued Cd (Cadmium)

$\mu \mathrm{g} / \mathrm{g}$

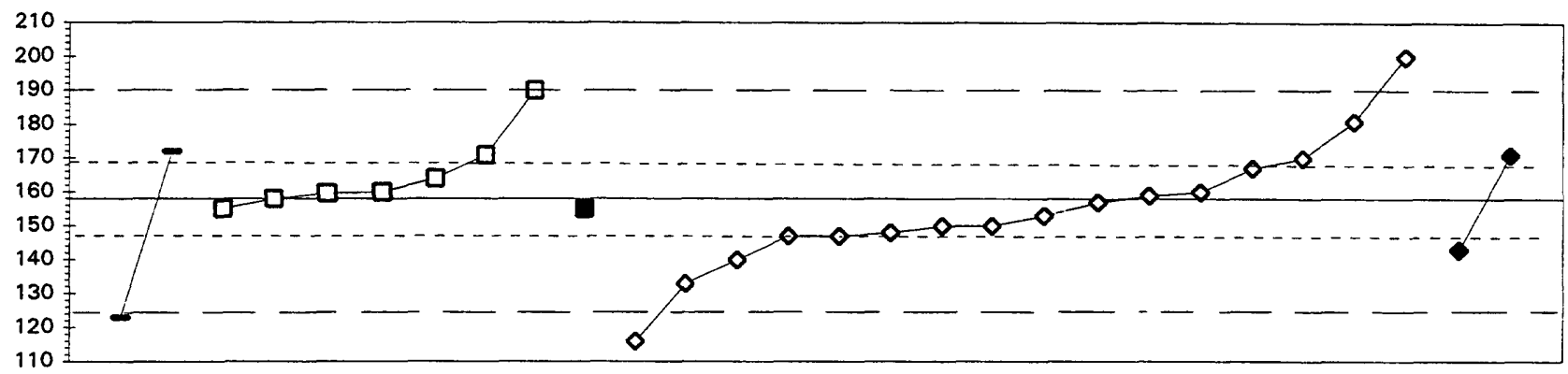

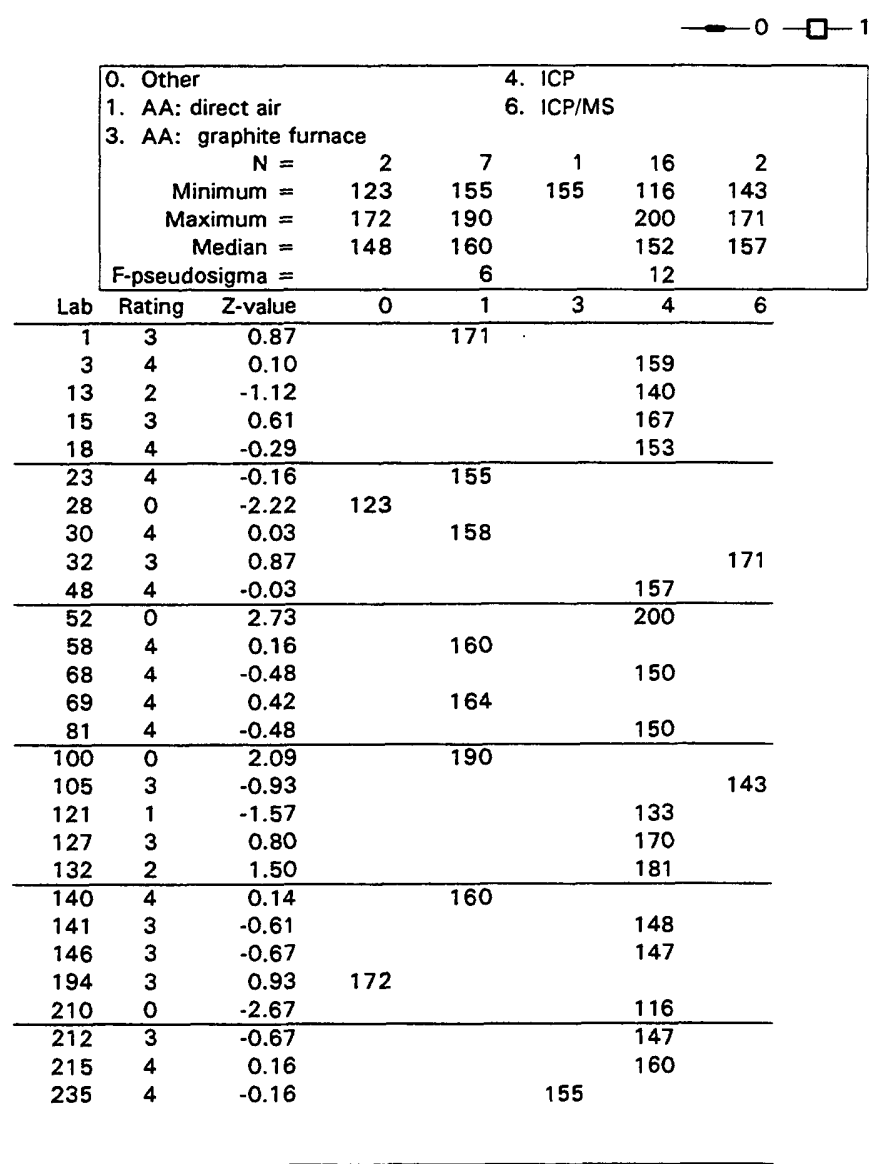


Table 18. Statistical summary of reported data for standard reference sediment sample SED-5 (bed material)--Continued Co (Cobalt)

\section{$\mu \mathrm{g} / \mathrm{g}$}

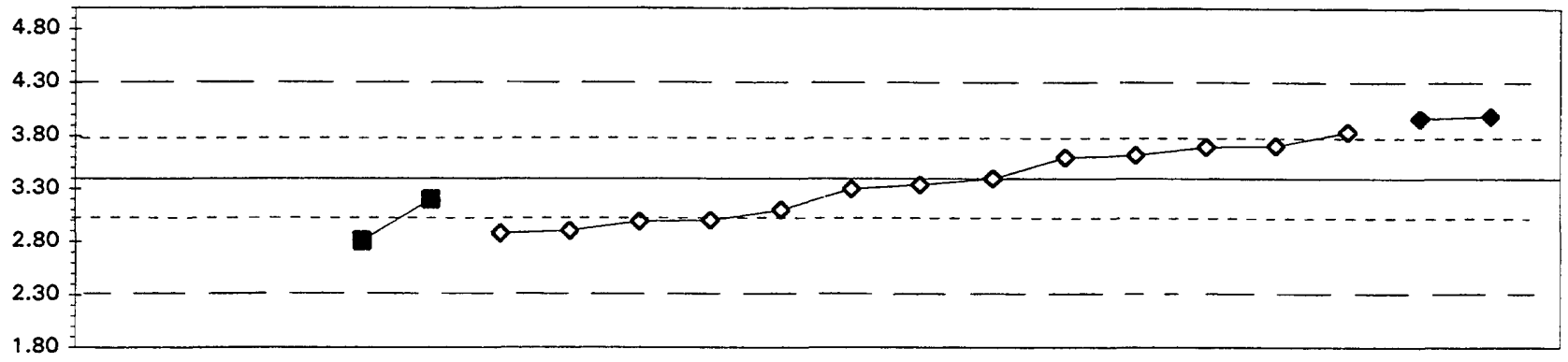

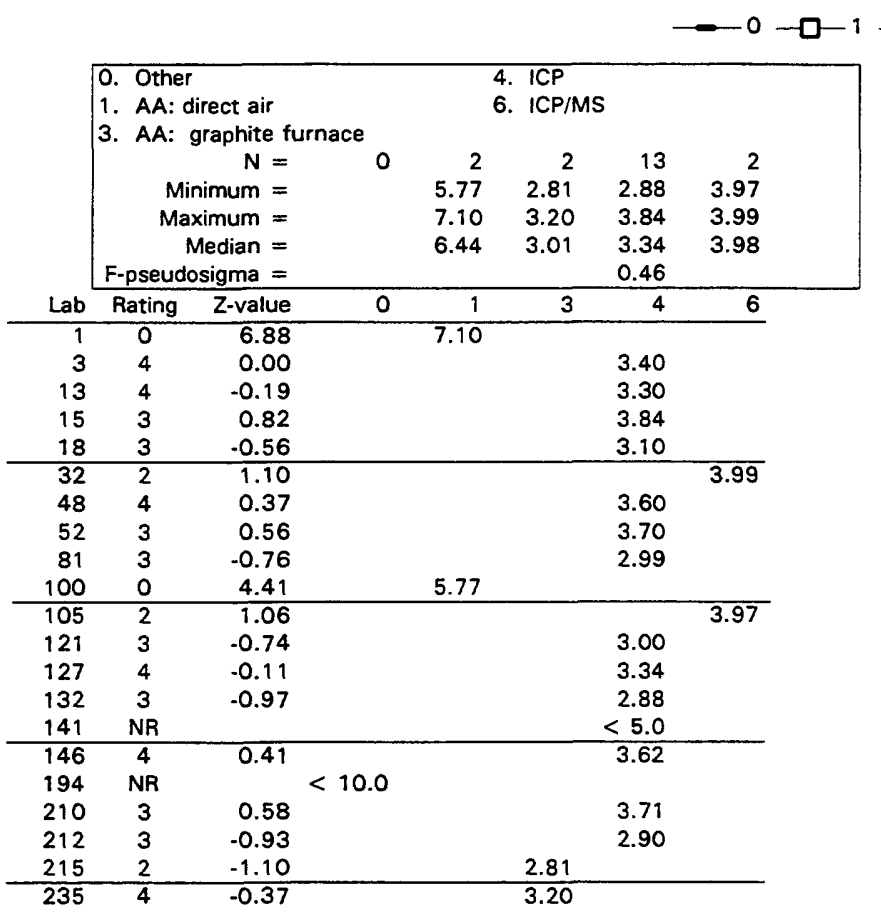


Table 18. Statistical summary of reported data for standard reference sediment sample SED-5 (bed material)--Continued $\mathrm{Cr}$ (Chromium)

$\mu \mathrm{g} / \mathrm{g}$

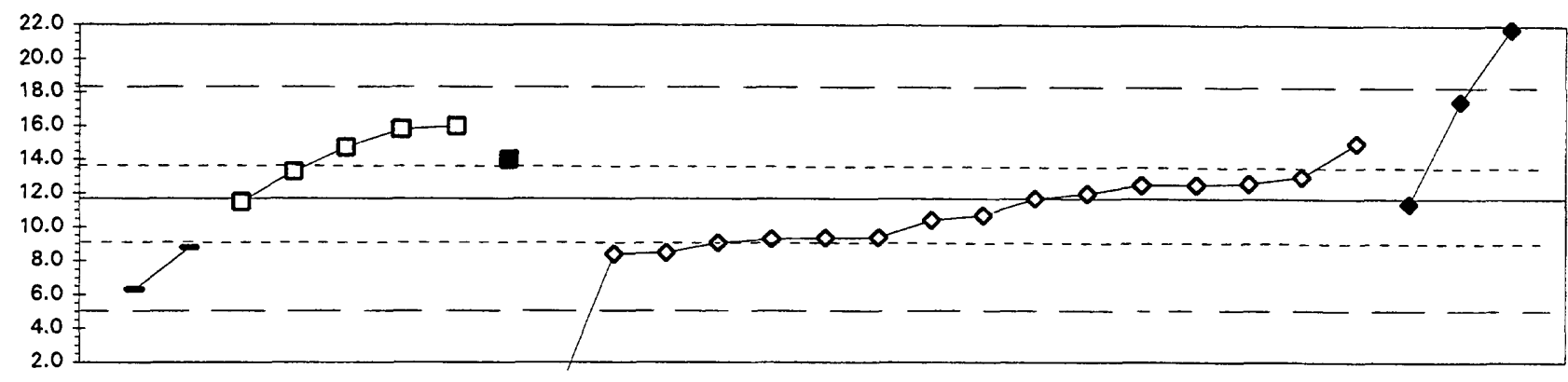

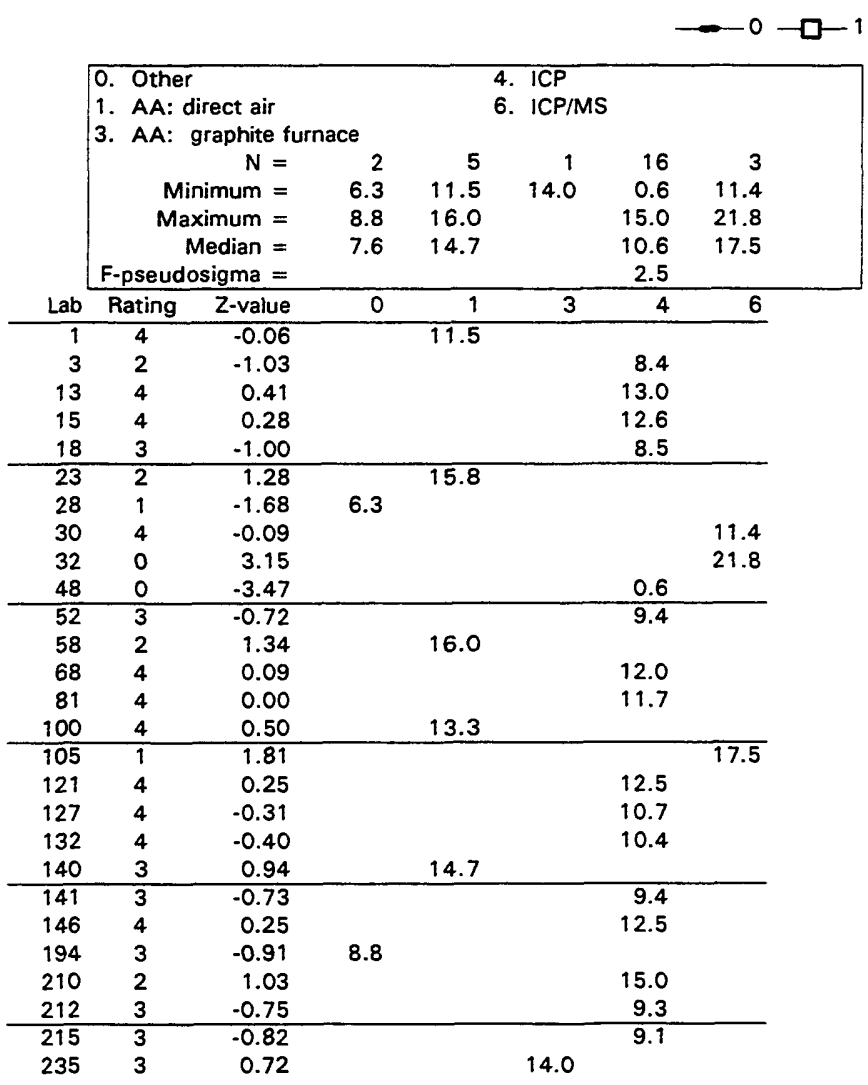


Table 18. Statistical summary of reported data for standard reference sediment sample SED-5 (bed material)--Continued $\mathrm{Cu}$ (Copper) $\mu \mathrm{g} / \mathrm{g}$

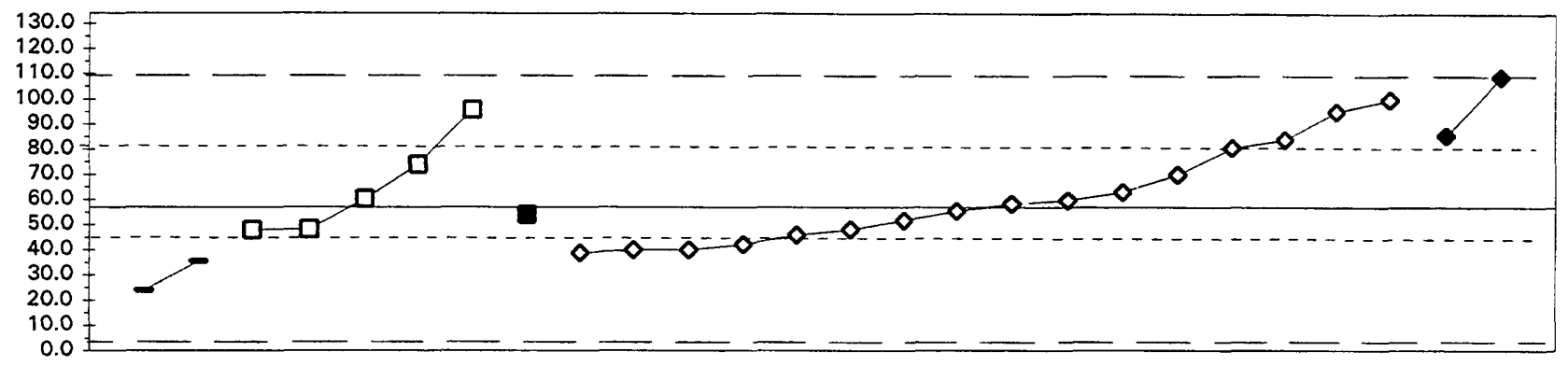

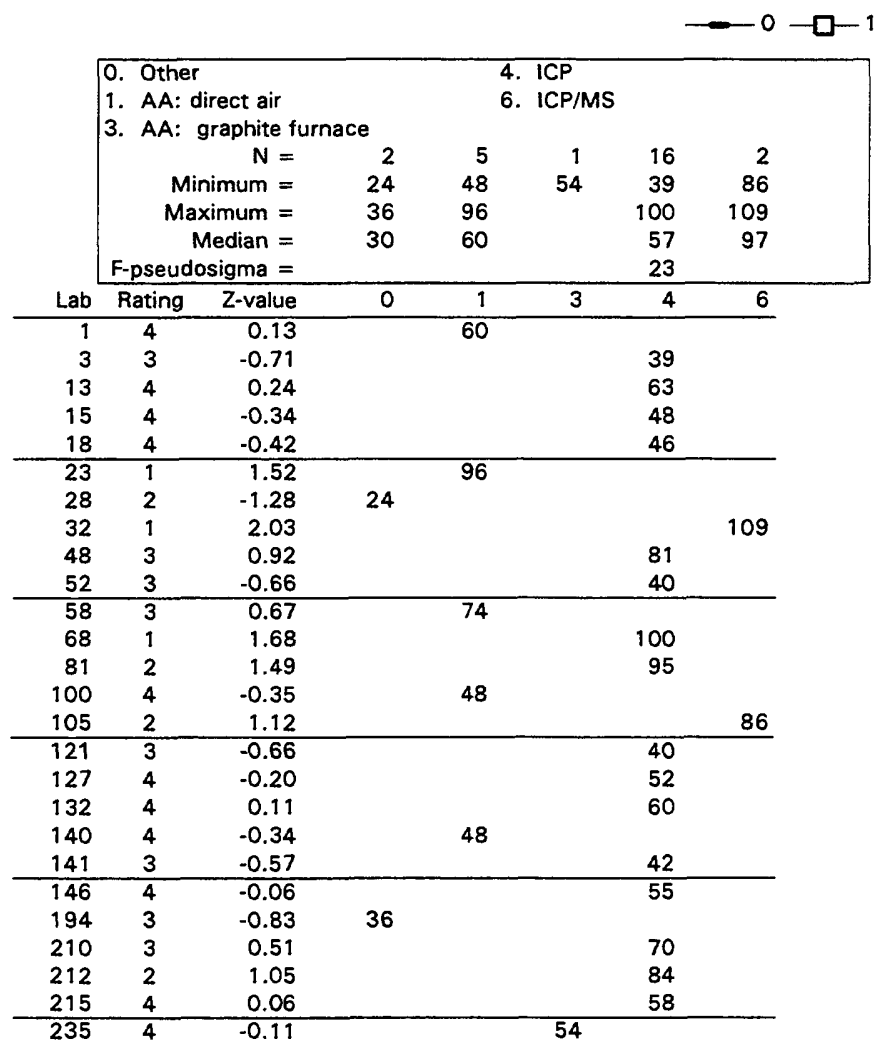


Table 18. Statistical summary of reported data for standard reference sediment sample SED-5 (bed material)--Continued $\mathrm{Fe}$ (Iron)

$\mu \mathrm{g} / \mathrm{g}$

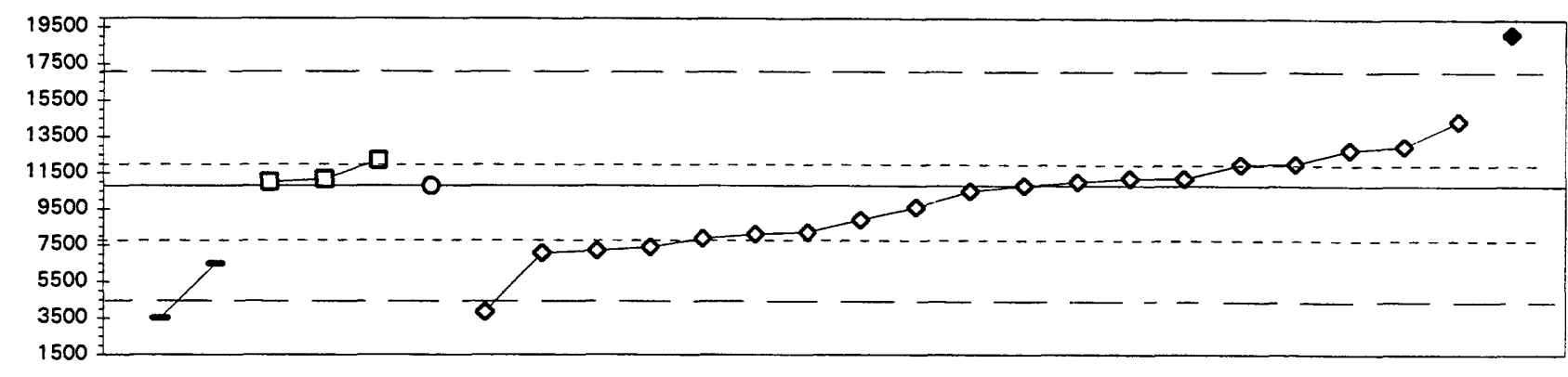

\begin{tabular}{|c|c|c|c|c|c|c|c|}
\hline \multirow[b]{2}{*}{ Lab } & \multicolumn{2}{|c|}{$\begin{array}{r}\text { 0. Other } \\
\text { 1. AA: direct air } \\
\text { 2. AA: direct nitrous } \\
\mathrm{N}= \\
\text { Minimum }= \\
\text { Maximum }= \\
\text { Median }= \\
\text { F-pseudosigma }=\end{array}$} & \multirow[t]{2}{*}{$\begin{array}{r}\text { oxide } \\
2 \\
3530 \\
6490 \\
5010 \\
0\end{array}$} & $\begin{array}{r}3 \\
11000 \\
12200 \\
11150\end{array}$ & $\begin{array}{l}\text { 4. ICP } \\
\text { 6. ICP/N }\end{array}$ & $\begin{array}{r}19 \\
3856 \\
14400 \\
10500 \\
2674\end{array}$ & $19200^{1}$ \\
\hline & Rating & Z-value & & 1 & 2 & 4 & 6 \\
\hline 1 & 4 & 0.46 & & 12200 & & & \\
\hline 3 & 2 & -1.18 & & & & 7210 & \\
\hline 13 & 3 & 0.72 & & & & 13000 & \\
\hline 15 & 4 & -0.10 & & & & 10500 & \\
\hline 18 & 2 & -1.22 & & & & 7080 & \\
\hline 28 & 0 & -2.38 & 3530 & & & & \\
\hline 32 & 0 & 2.75 & & & & & 19200 \\
\hline 48 & 4 & 0.07 & & & & 11004 & \\
\hline 52 & 3 & -0.89 & & & & 8100 & \\
\hline 58 & 4 & 0.07 & & 11000 & & & \\
\hline 68 & 3 & -0.62 & & & & 8900 & \\
\hline 81 & 4 & 0.00 & & & & 10800 & \\
\hline 100 & 0 & -2.28 & & & & 3856 & \\
\hline 105 & 3 & 0.66 & & & & 12800 & \\
\hline 121 & 4 & 0.39 & & & & 12000 & \\
\hline 127 & 4 & -0.40 & & & & 9590 & \\
\hline 132 & 4 & 0.38 & & & & 11974 & \\
\hline 140 & 4 & 0.11 & & 11150 & & & \\
\hline 141 & 2 & -1.12 & & & & 7390 & \\
\hline 146 & 4 & 0.13 & & & & 11200 & \\
\hline 154 & 2 & 1.18 & & & & 14400 & \\
\hline 194 & 2 & -1.41 & 6490 & & & & \\
\hline 210 & 4 & 0.13 & & & & 11200 & \\
\hline 212 & 3 & -0.85 & & & & 8200 & \\
\hline 215 & 3 & -0.96 & & & & 7860 & \\
\hline 235 & 4 & 0.00 & & & 10800 & & \\
\hline
\end{tabular}


Table 18. Statistical summary of reported data for standard reference sediment sample SED-5 (bed material)--Continued K (Potassium)

$\mathrm{mg} / \mathrm{g}$

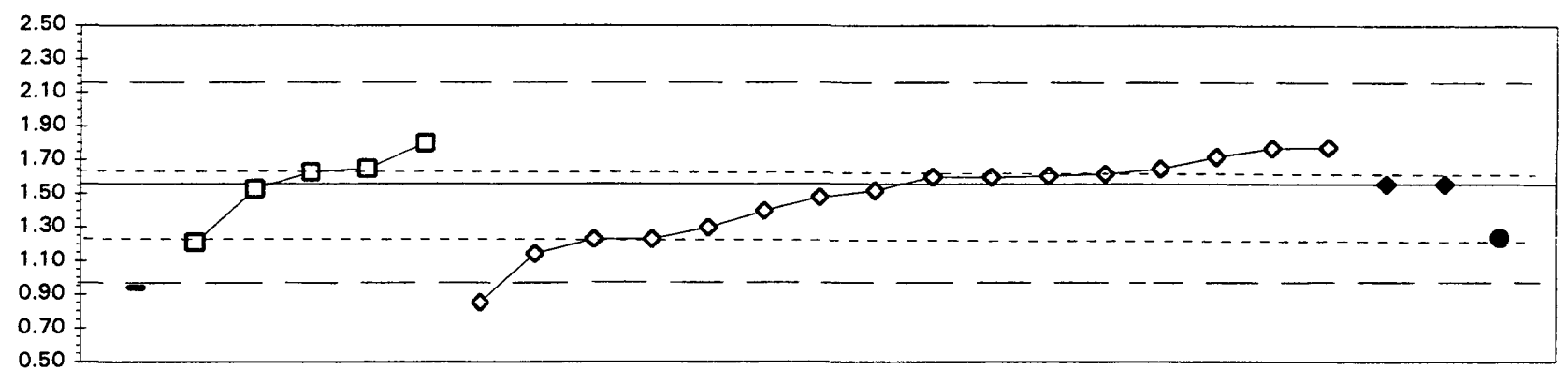

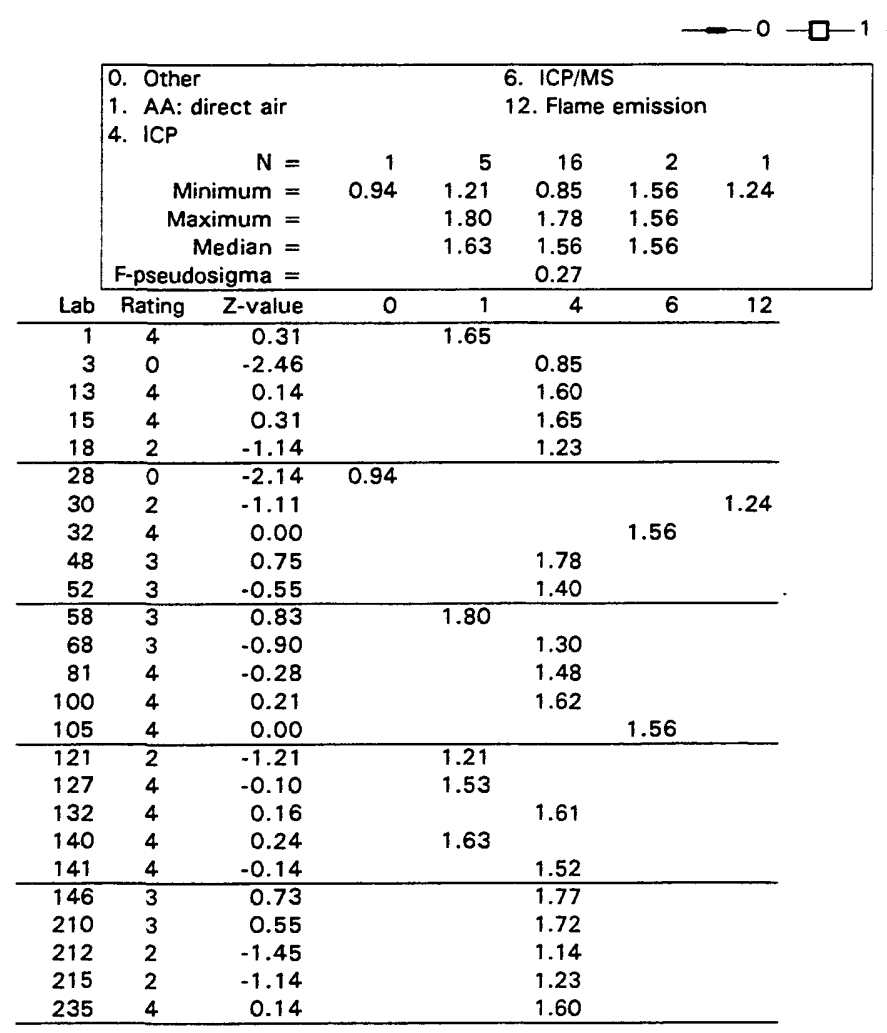


Table 18. Statistical summary of reported data for standard reference sediment sample SED-5 (bed material)--Continued Li (Lithium)

$\mu \mathrm{g} / \mathrm{g}$

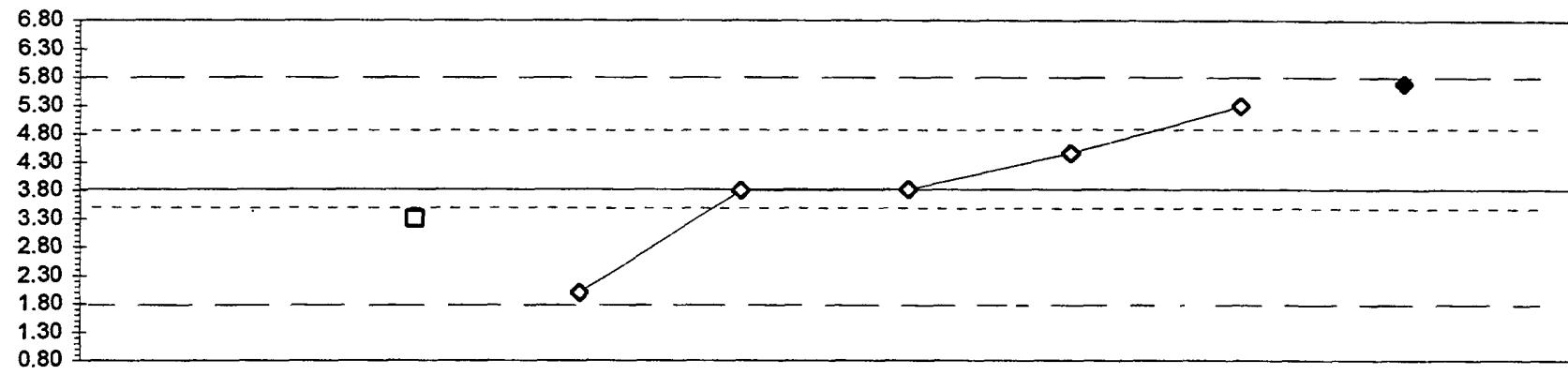

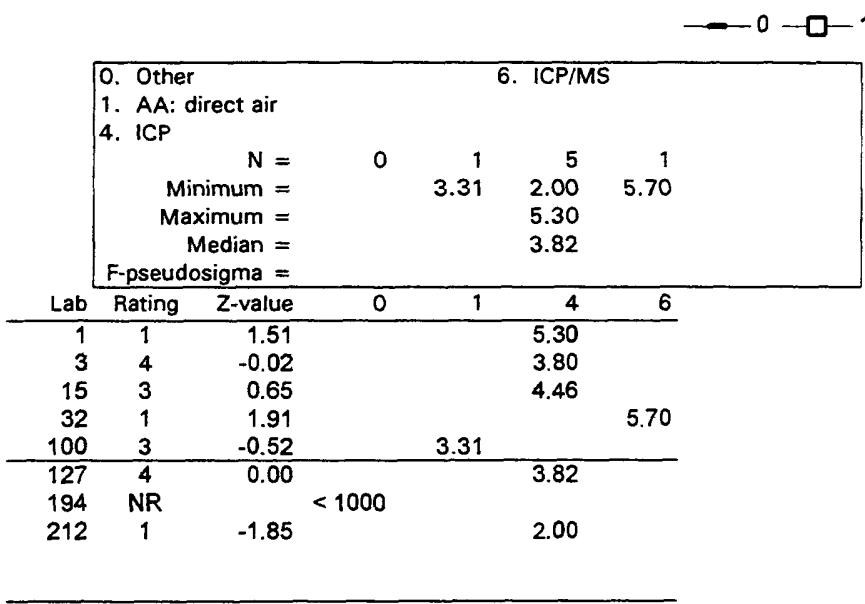


Table 18. Statistical summary of reported data for standard reference sediment sample SED-5 (bed material)--Continued Mg (Magnesium) $\mathrm{mg} / \mathrm{g}$

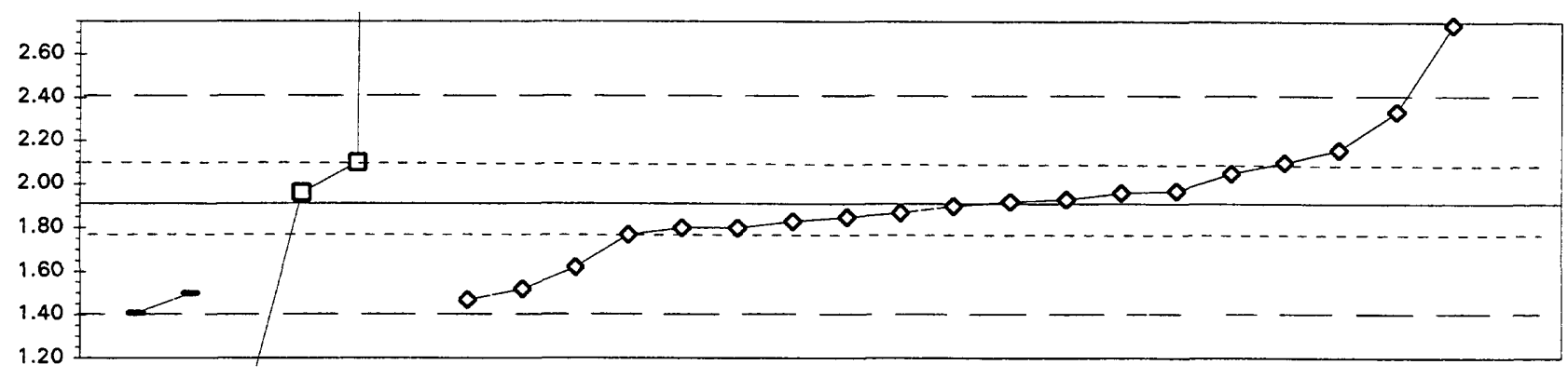

\begin{tabular}{|c|c|c|c|c|c|c|}
\hline \multirow[b]{2}{*}{ Lab } & $\begin{array}{l}\text { 0. Othe } \\
\text { 1. AA: } \\
\text { 4. ICP } \\
\text { F-pseud }\end{array}$ & $\begin{array}{r}\text { rect air } \\
\mathrm{N}= \\
\text { imum }= \\
\text { imum }= \\
\text { ledian }= \\
\text { sigma }=\end{array}$ & $\begin{array}{r}2 \\
1.41 \\
1.50 \\
1.46\end{array}$ & $\begin{array}{r}4 \\
0.94 \\
52.00 \\
2.03\end{array}$ & $\begin{array}{r}19 \\
1.47 \\
2.74 \\
1.90 \\
0.16 \\
\end{array}$ & $\begin{array}{r}1 \\
3.20\end{array}$ \\
\hline & Rating & $\mathrm{Z}$-value & 0 & 1 & 4 & 6 \\
\hline 1 & 4 & 0.20 & & 1.96 & & \\
\hline 3 & 1 & -1.59 & & & 1.52 & \\
\hline 13 & 4 & -0.04 & & & 1.90 & \\
\hline 15 & 2 & 1.02 & & & 2.16 & \\
\hline 18 & 2 & -1.19 & & & 1.62 & \\
\hline 23 & 0 & -3.97 & & 0.94 & & \\
\hline 28 & 1 & -2.04 & 1.41 & & & \\
\hline 32 & 0 & 5.27 & & & & 3.20 \\
\hline 48 & 1 & 1.75 & & & 2.34 & \\
\hline 52 & 4 & -0.45 & & & 1.80 & \\
\hline 58 & 0 & 204.76 & & 52.00 & & \\
\hline 68 & 4 & -0.45 & & & 1.80 & \\
\hline 81 & 4 & -0.25 & & & 1.85 & \\
\hline 100 & 4 & 0.04 & & & 1.92 & \\
\hline 105 & 4 & 0.08 & & & 1.93 & \\
\hline 121 & 4 & 0.20 & & & 1.96 & \\
\hline 127 & 4 & 0.25 & & & 1.97 & \\
\hline 132 & 0 & 3.38 & & & 2.74 & \\
\hline 140 & 3 & 0.78 & & 2.10 & & \\
\hline 141 & 4 & -0.33 & & & 1.83 & \\
\hline 146 & 3 & 0.57 & & & 2.05 & \\
\hline 194 & 1 & -1.68 & 1.50 & & & \\
\hline 210 & 3 & -0.57 & & & 1.77 & \\
\hline 212 & 1 & -1.80 & & & 1.47 & \\
\hline 215 & 4 & -0.16 & & & 1.87 & \\
\hline 235 & 3 & 0.78 & & & 2.10 & \\
\hline
\end{tabular}


Table 18. Statistical summary of reported data for standard reference sediment sample SED-5 (bed material)--Continued Mn (Manganese) $\mu \mathrm{g} / \mathrm{g}$

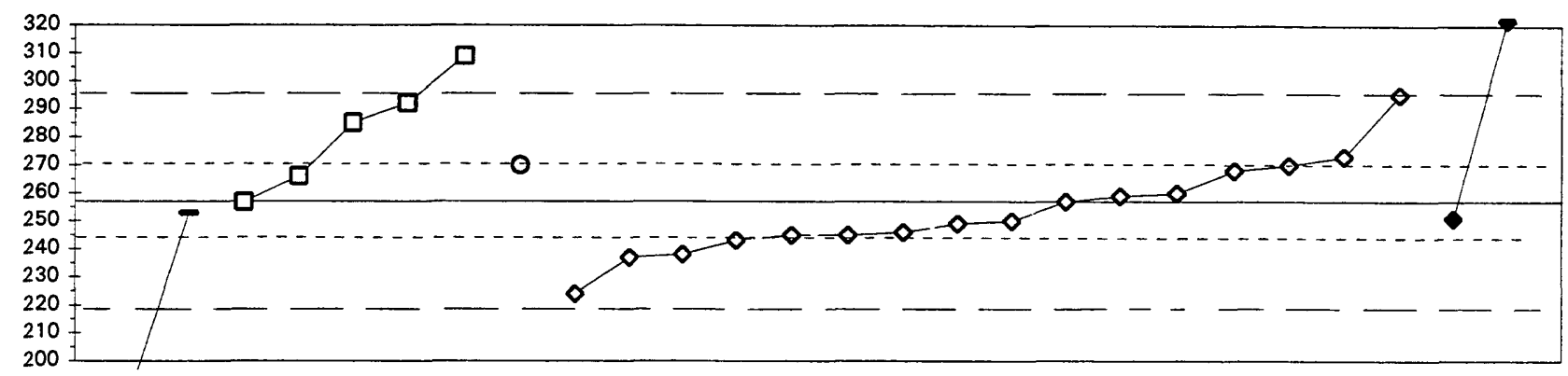

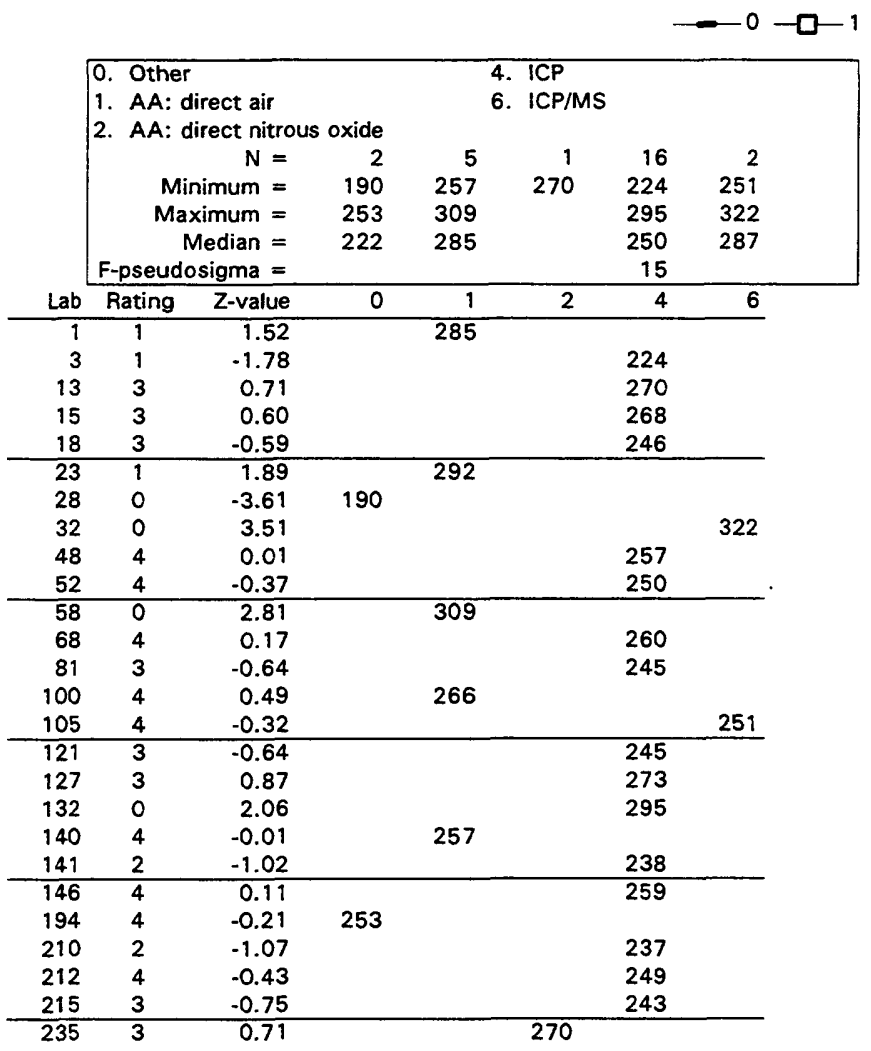


Table 18. Statistical summary of reported data for standard reference sediment sample SED-5 (bed material)--Continued Mo (Molybdenum)

$\mu \mathrm{g} / \mathrm{g}$

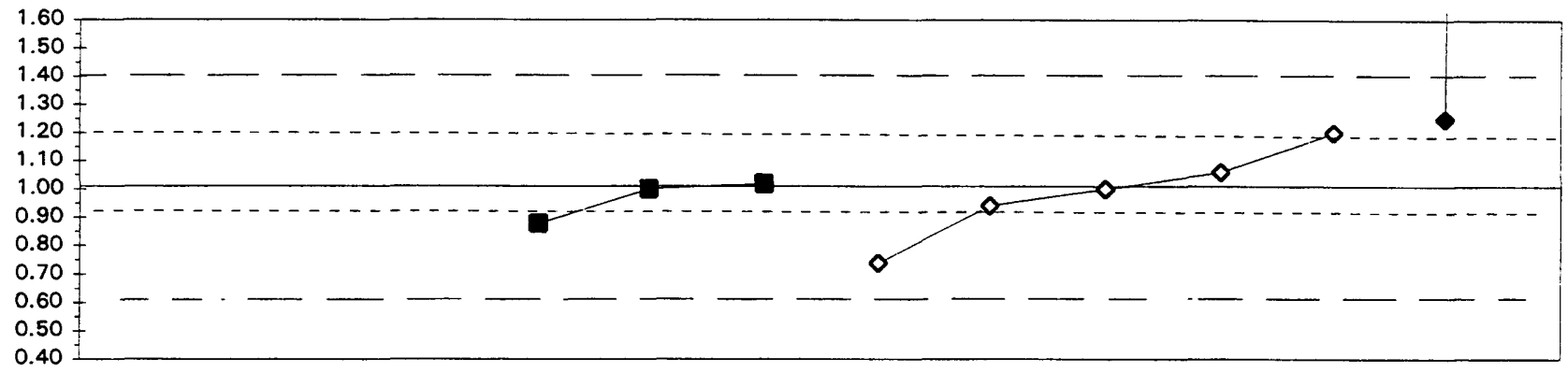

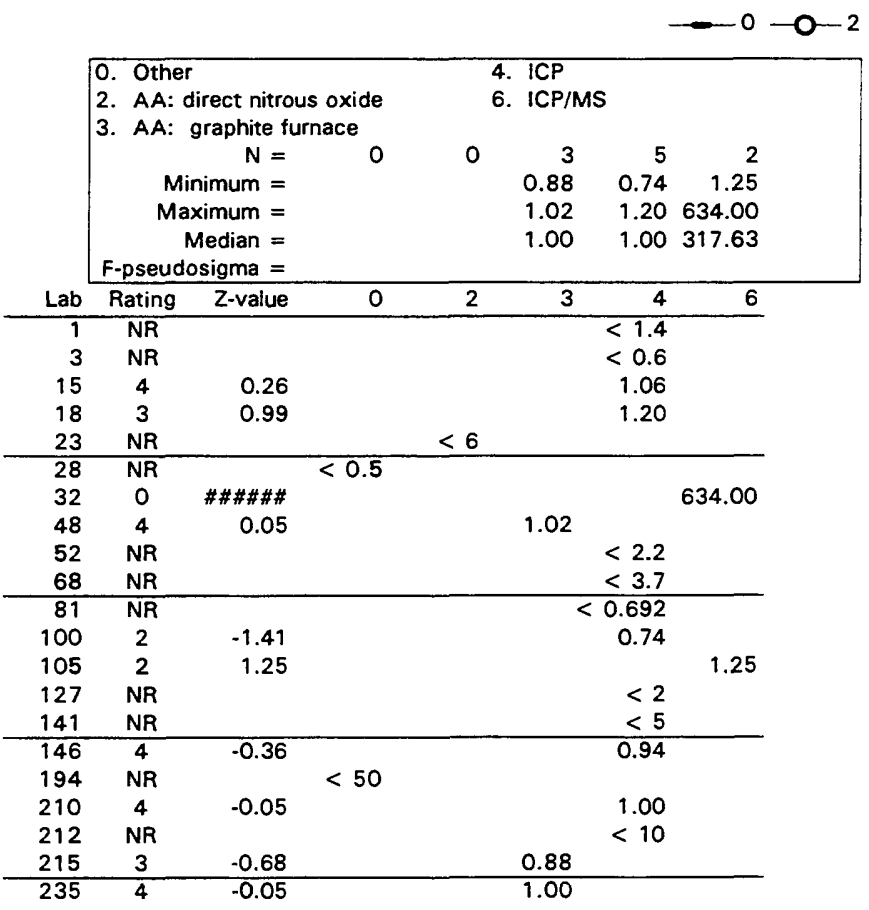


Table 18. Statistical summary of reported data for standard reference sediment sample SED-5 (bed material)--Continued $\mathrm{Na}$ (Sodium) $\mathrm{mg} / \mathrm{g}$

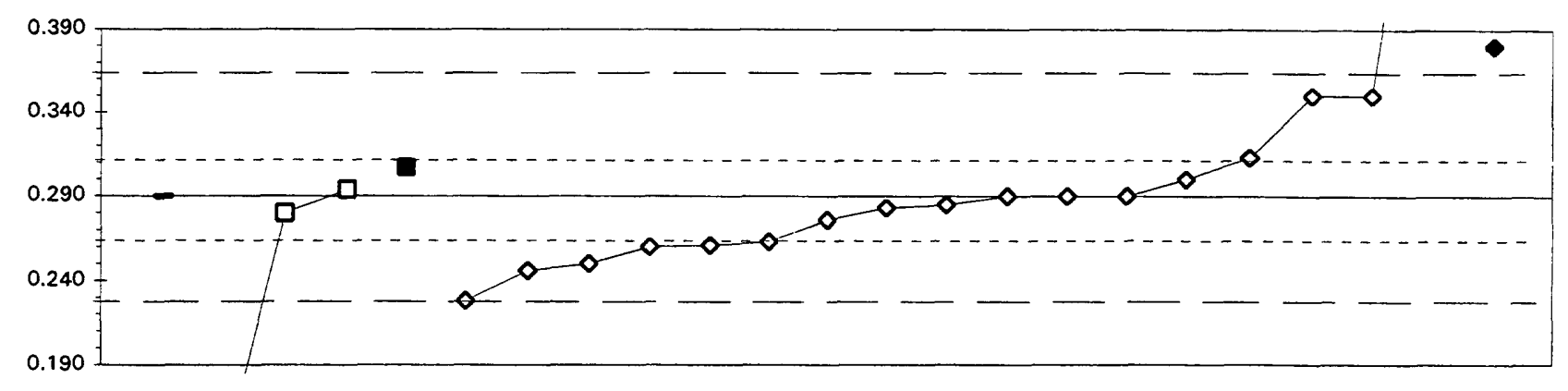

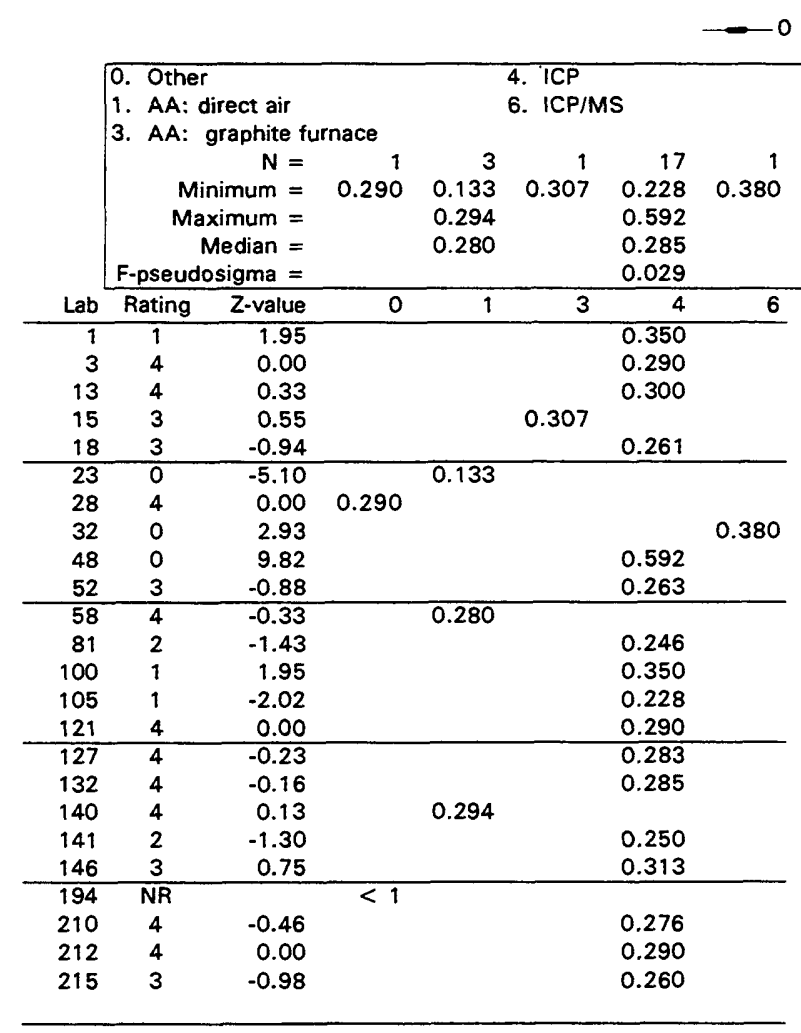


Table 18. Statistical summary of reported data for standard reference sediment sample SED-5 (bed material)--Continued $\mathrm{Ni}$ (Nickel) $\mu \mathrm{g} / \mathrm{g}$

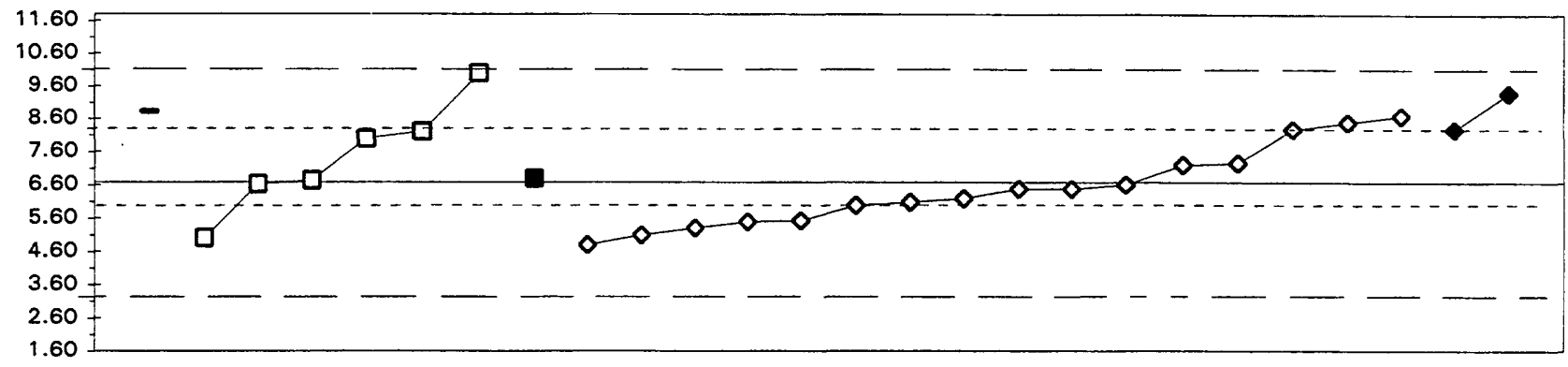

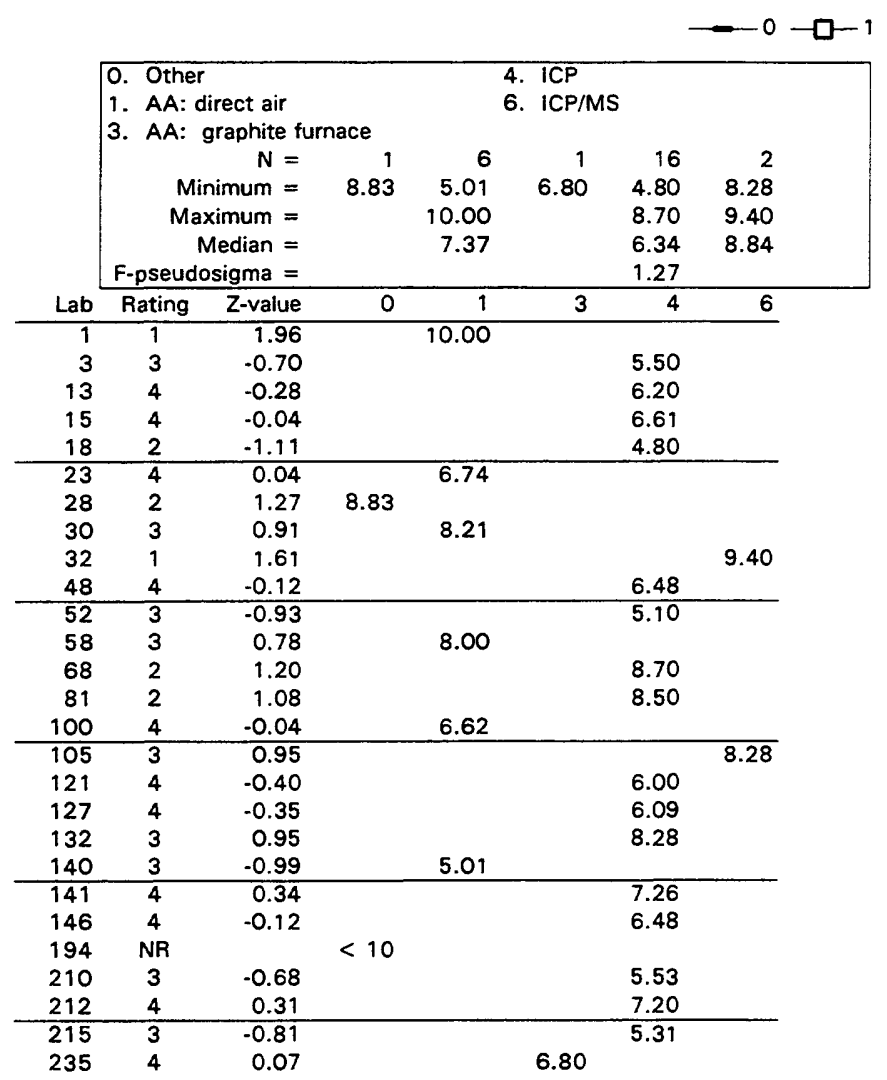


Table 18. Statistical summary of reported data for standard reference sediment sample SED-5 (bed material)--Continued $\mathrm{Pb}$ (Lead) $\mu \mathrm{g} / \mathrm{g}$

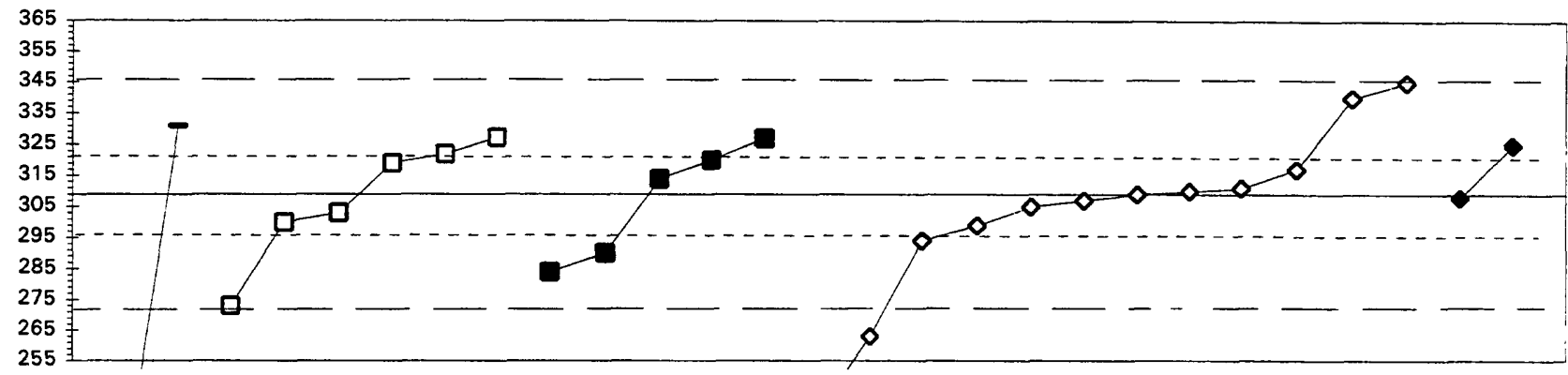

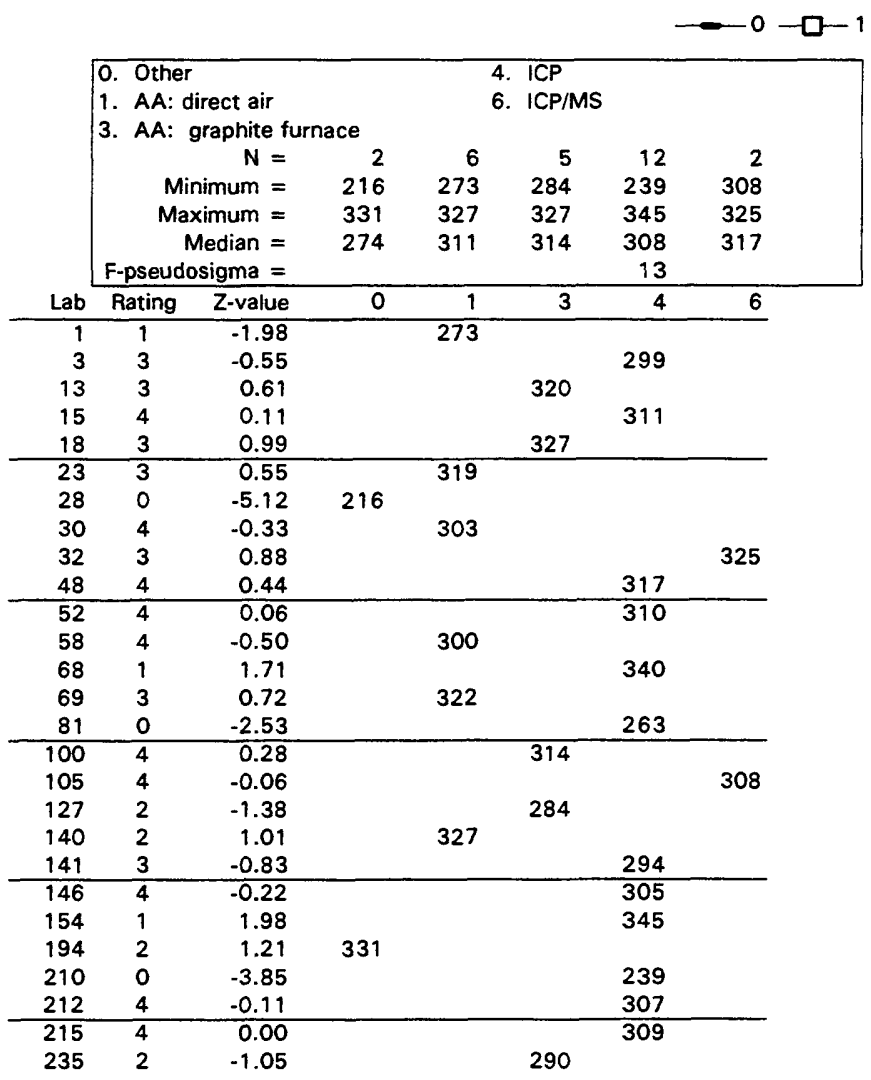


Table 18. Statistical summary of reported data for standard reference sediment sample SED-5 (bed material)--Continued $\mathrm{Sb}$ (Antimony) $\mu \mathrm{g} / \mathrm{g}$

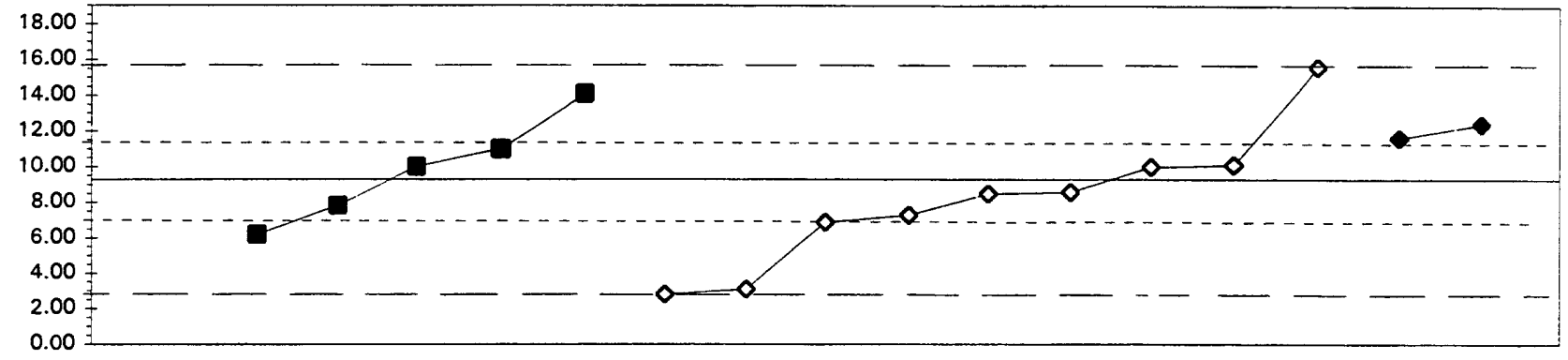

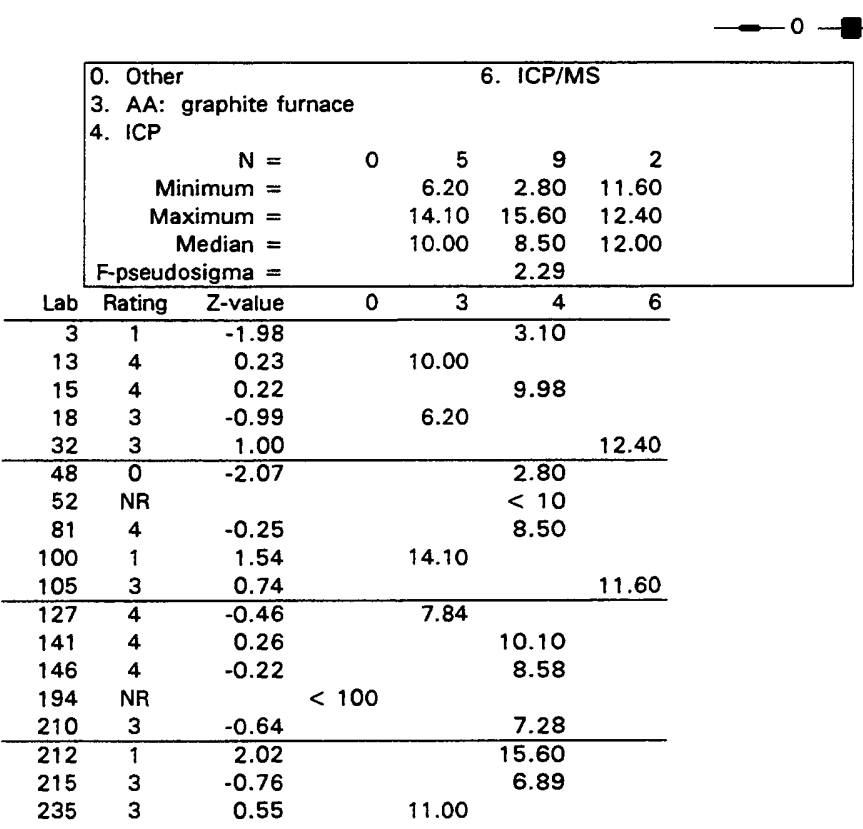


Table 18. Statistical summary of reported data for standard reference sediment sample SED-5 (bed material)--Continued Se (Selenium) $\mu \mathrm{g} / \mathrm{g}$

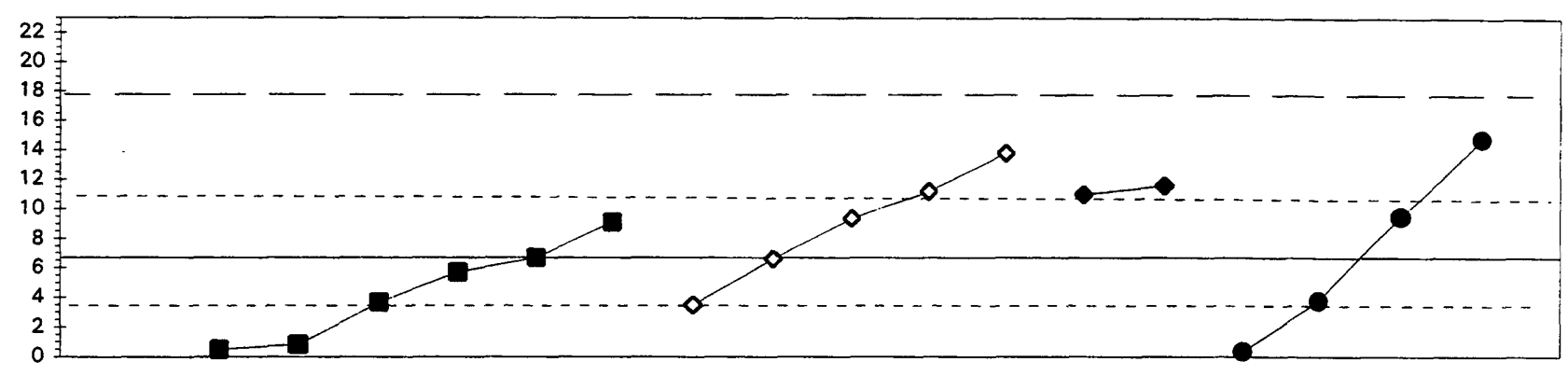

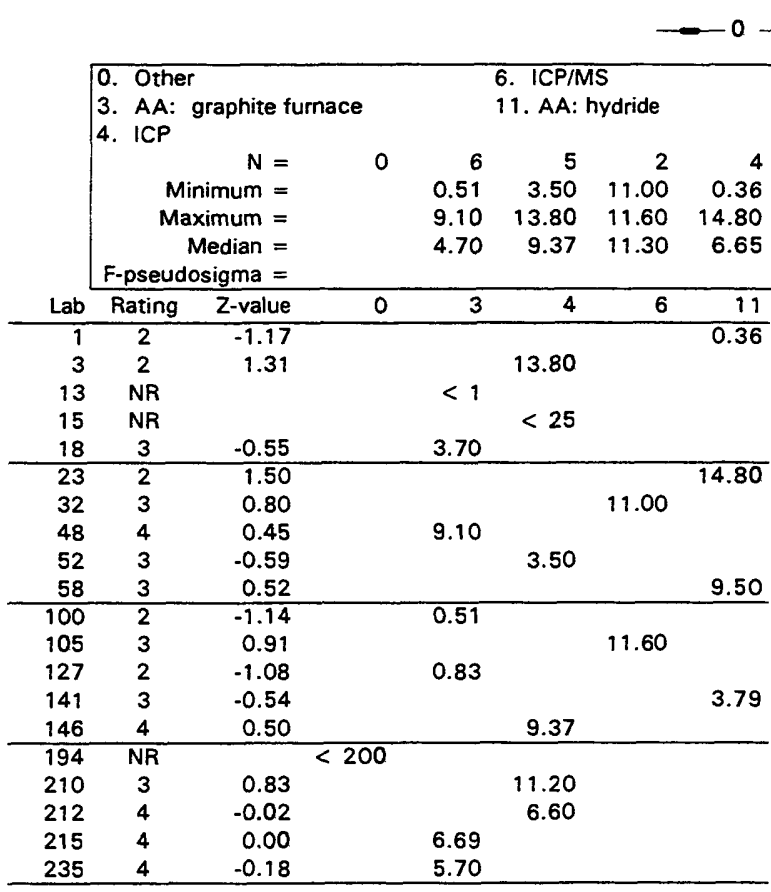


Table 18. Statistical summary of reported data for standard reference sediment sample SED-5 (bed material)--Continued $\mathrm{SiO} 2$ (Silica)

$\mathrm{mg} / \mathrm{g}$

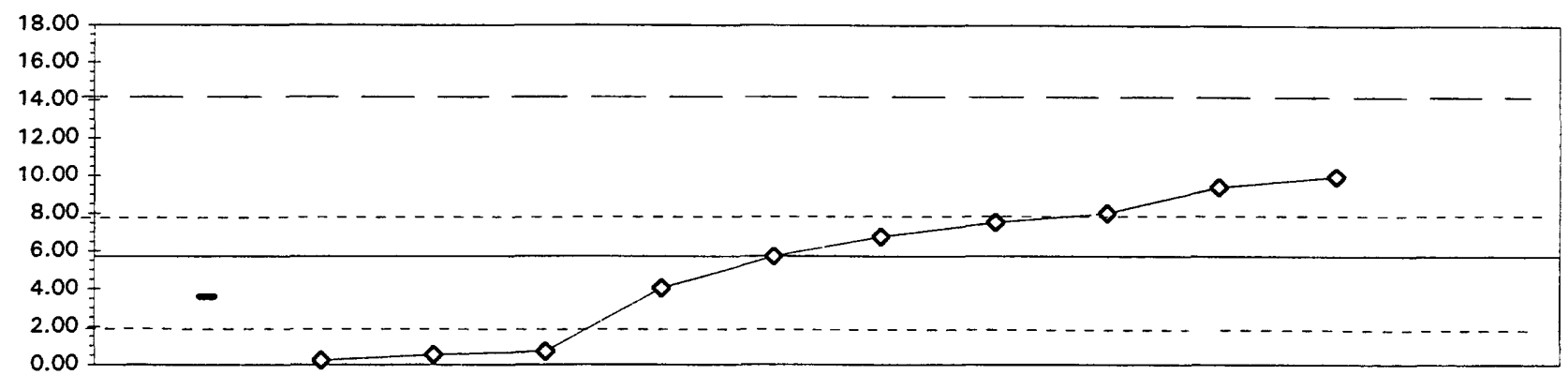

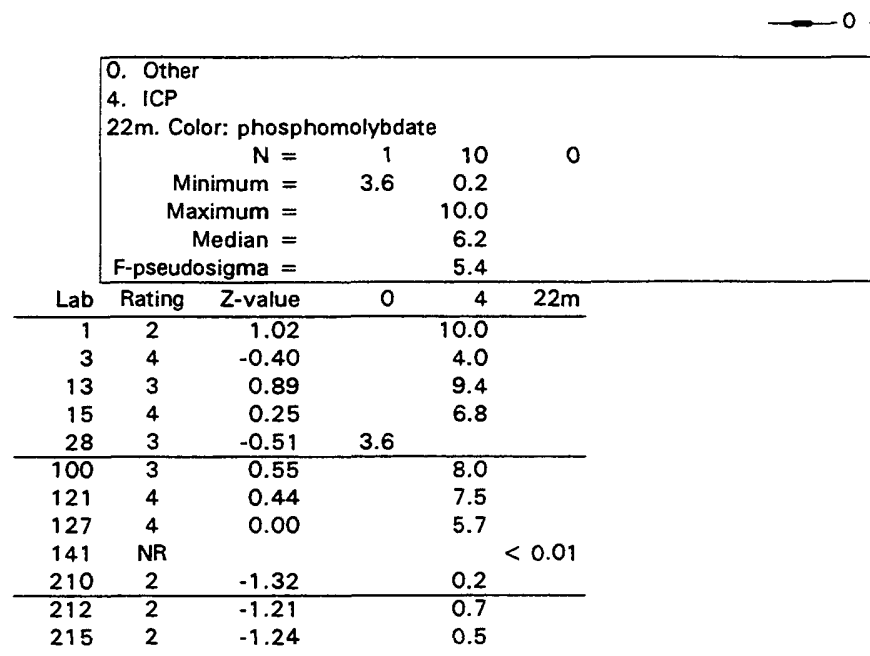


Table 18. Statistical summary of reported data for standard reference sediment sample SED-5 (bed material)--Continued Sr (Strontium)

$\mu \mathrm{g} / \mathrm{g}$

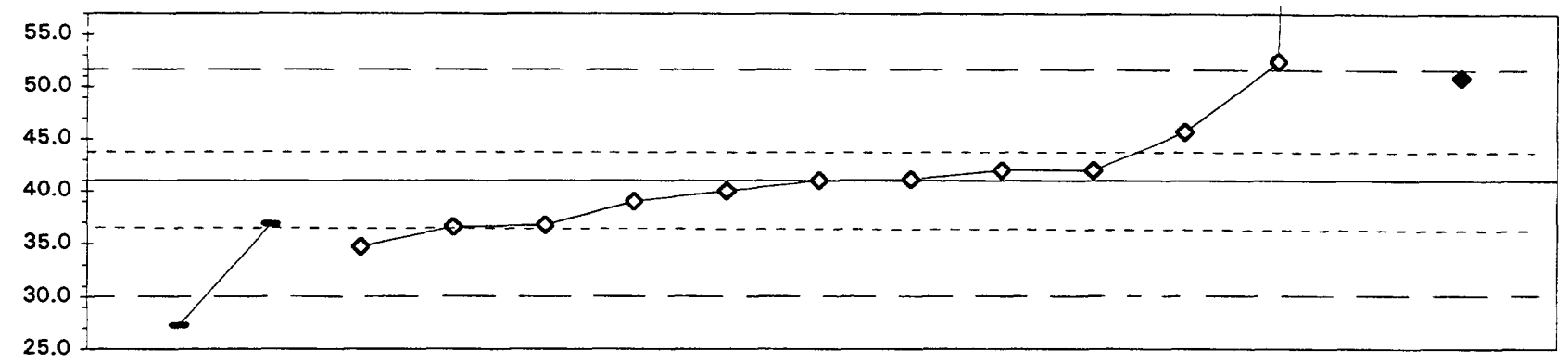

\begin{tabular}{|c|c|c|c|c|c|}
\hline \multirow[b]{2}{*}{ Lab } & $\begin{array}{l}\text { 0. Other } \\
\text { 4. ICP } \\
\text { 6. ICP/N } \\
\text { F-pseud } \\
\end{array}$ & $\begin{aligned} \mathrm{N} & = \\
\text { imum } & = \\
\text { imum } & = \\
\text { edian } & = \\
\text { igma } & =\end{aligned}$ & $\begin{array}{r}2 \\
27.3 \\
36.9 \\
32.1\end{array}$ & $\begin{array}{r}12 \\
34.7 \\
409.0 \\
41.0 \\
4.4\end{array}$ & $\begin{array}{r}1 \\
50.9\end{array}$ \\
\hline & Rating & $Z$-value & 0 & 4 & 6 \\
\hline 1 & 0 & 2.20 & & 52.4 & \\
\hline 3 & 3 & -0.85 & & 36.6 & \\
\hline 15 & 4 & 0.19 & & 42.0 & \\
\hline 18 & 4 & -0.39 & & 39.0 & \\
\hline 28 & 0 & -2.64 & 27.3 & & \\
\hline 32 & 1 & 1.91 & & & 50.9 \\
\hline 52 & 4 & -0.19 & & 40.0 & \\
\hline 81 & 4 & 0.02 & & 41.1 & \\
\hline 100 & 2 & -1.21 & & 34.7 & \\
\hline 121 & 4 & 0.00 & & 41.0 & \\
\hline 127 & 3 & 0.91 & & 45.7 & \\
\hline 194 & 3 & -0.79 & 36.9 & & \\
\hline 210 & 0 & 70.92 & & 409.0 & \\
\hline 212 & 3 & -0.81 & & 36.8 & \\
\hline 235 & 4 & 0.19 & & 42.0 & \\
\hline
\end{tabular}


Table 18. Statistical summary of reported data for standard reference sediment sample SED-5 (bed material)--Continued V (Vanadium) $\mu \mathrm{g} / \mathrm{g}$

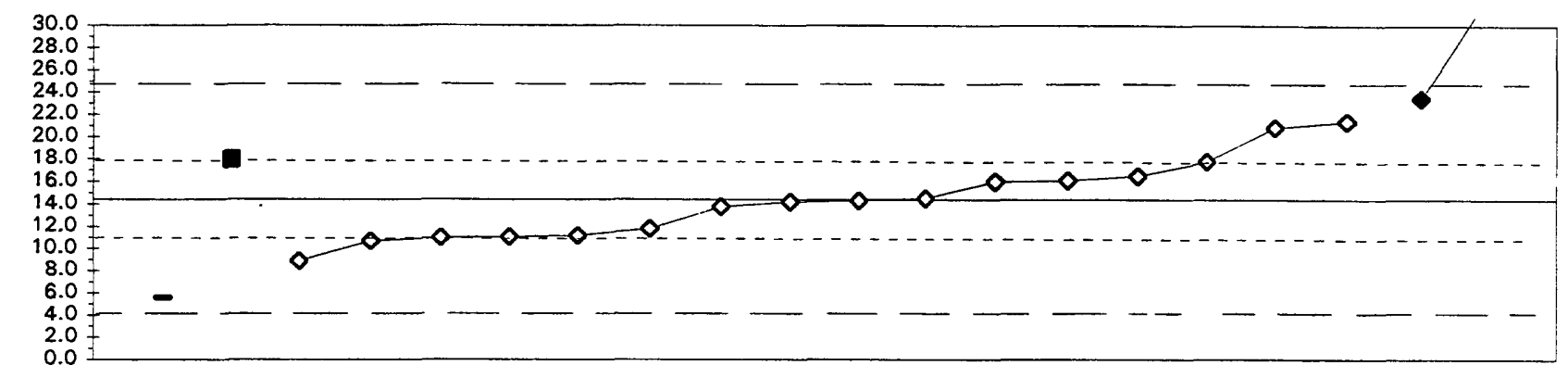

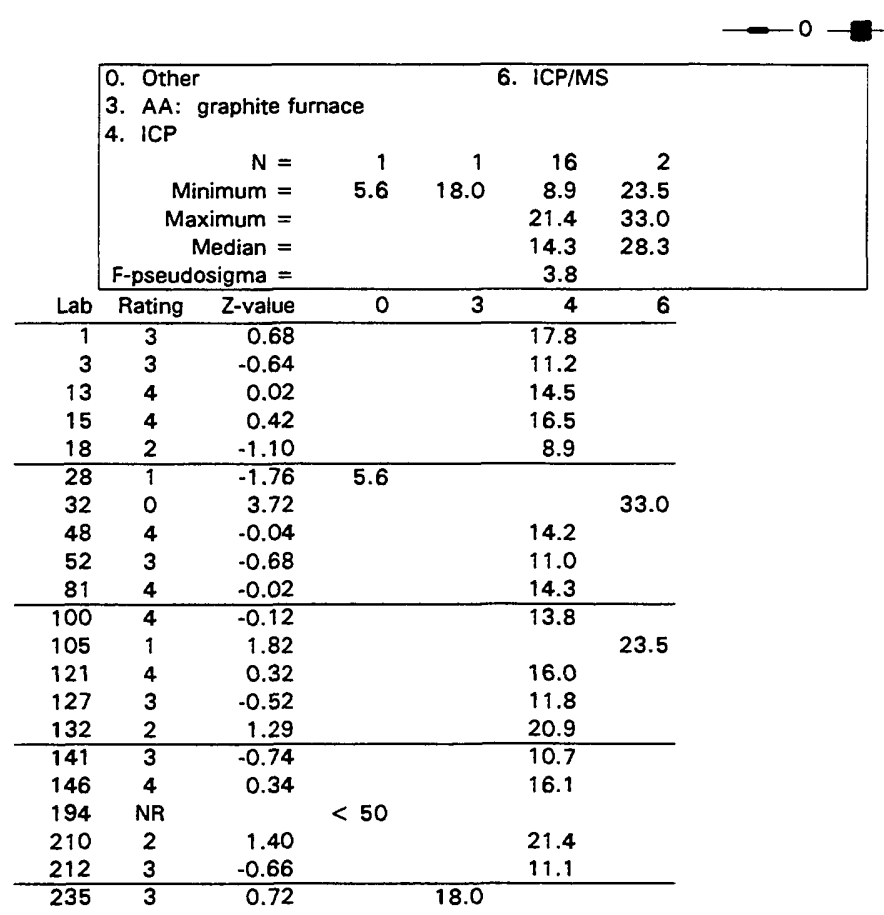


Table 18. Statistical summary of reported data for standard reference sediment sample SED-5 (bed material)--Continued Zn (Zinc) $\mu \mathrm{g} / \mathrm{g}$

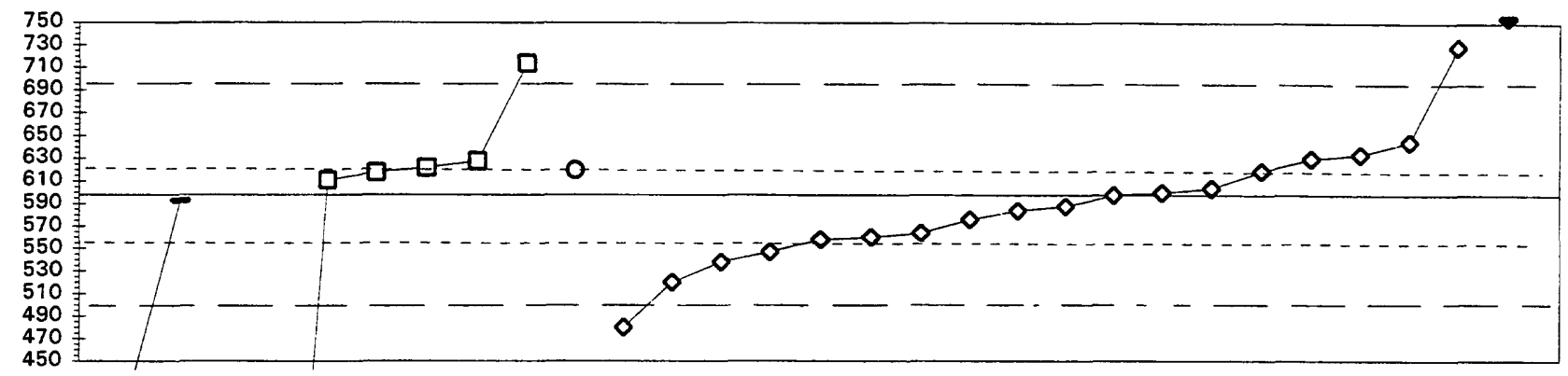

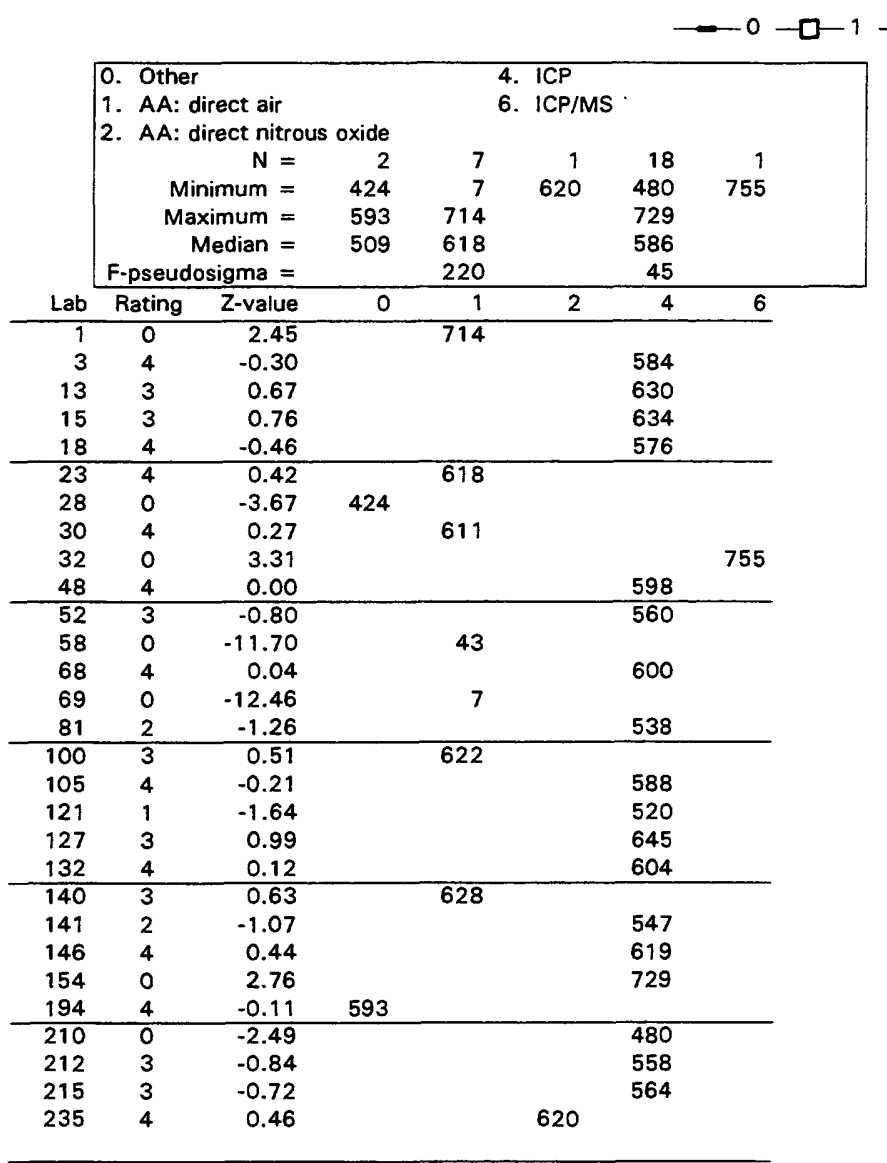


[MPV, most probable value; ug/L, microgram per liter; $\mathrm{mg} / \mathrm{L}$, milligram per liter; uS/cm. microsiemen per centimeter at 25 degrees Celsius]

\begin{tabular}{|c|c|c|c|c|c|}
\hline \multicolumn{6}{|c|}{ T-135 (trace constituents) } \\
\hline Analyte & MPV & F-pseudosigma & Analyte & MPV & F-pseudosiama \\
\hline $\mathrm{Ag}$ & $9.81 \mu \mathrm{g} / \mathrm{L}$ & 1.05 & L & $73.7 \mu \mathrm{g} / \mathrm{L}$ & 5.2 \\
\hline Al & $10.5 \mu \mathrm{g} / \mathrm{L}$ & 6.8 & $\mathrm{Mg}$ & $2.00 \mathrm{mg} / \mathrm{L}$ & 0.09 \\
\hline As & $10.0 \mu \mathrm{g} / \mathrm{L}$ & 1.1 & $\mathrm{Mn}$ & $423 \mu \mathrm{g} / \mathrm{L}$ & 20 \\
\hline B & $13.1 \mu \mathrm{g} / \mathrm{L}$ & 11.1 & Mo & $63.0 \mu \mathrm{g} / \mathrm{L}$ & 5.1 \\
\hline $\mathrm{Ba}$ & $67.8 \mu \mathrm{g} / \mathrm{L}$ & 4.3 & $\mathrm{Na}$ & $30.8 \mathrm{mg} / \mathrm{L}$ & 1.2 \\
\hline $\mathrm{Be}$ & $59.0 \mu \mathrm{g} / \mathrm{L}$ & 2.6 & $\mathrm{Ni}$ & $65.6 \mu \mathrm{g} / \mathrm{L}$ & 5.0 \\
\hline $\mathrm{Ca}$ & $10.4 \mathrm{mg} / \mathrm{L}$ & 0.6 & $\mathrm{~Pb}$ & $103.0 \mu \mathrm{g} / \mathrm{L}$ & 7.0 \\
\hline Cd & $50.5 \mu \mathrm{g} / \mathrm{L}$ & 3.2 & $\mathrm{Sb}$ & $76.3 \mu \mathrm{g} / \mathrm{L}$ & 8.7 \\
\hline Co & $40.0 \mu \mathrm{g} / \mathrm{L}$ & 2.6 & $\mathrm{Se}$ & $10.0 \mu \mathrm{g} / \mathrm{L}$ & 1.4 \\
\hline $\mathrm{Cr}$ & $79.0 \mu \mathrm{g} / \mathrm{L}$ & 5.5 & $\mathrm{SiO} 2$ & $4.28 \mathrm{mg} / \mathrm{L}$ & 0.31 \\
\hline $\mathrm{Cu}$ & $62.0 \mu \mathrm{g} / \mathrm{L}$ & 4.2 & $\mathrm{Sr}$ & $46.0 \mu \mathrm{g} / \mathrm{L}$ & 2.3 \\
\hline $\mathrm{Fe}$ & $228 \mu \mathrm{g} / \mathrm{L}$ & 11 & $\mathrm{v}$ & $52.8 \mu \mathrm{g} / \mathrm{L}$ & 3.6 \\
\hline $\mathrm{K}$ & $0.96 \mathrm{mg} / \mathrm{L}$ & 0.09 & $\mathrm{Zn}$ & $48.2 \mu \mathrm{g} / \mathrm{L}$ & $\begin{array}{c}4.7 \\
\cdots . .\end{array}$ \\
\hline \multicolumn{6}{|c|}{ M-134 (major constituents) } \\
\hline Analyte & $\underline{\text { MPV }}$ & F-pseudosigma & Analyte & MPV & F-pseudosigma \\
\hline Alkalinity & $62.9 \mathrm{mg} / \mathrm{L}$ & 1.6 & $\mathrm{Na}$ & $60.7 \mathrm{mg} / \mathrm{L}$ & 2.4 \\
\hline B & $33.7 \mathrm{mg} / \mathrm{L}$ & 8.5 & total P & $0.010 \mathrm{mg} / \mathrm{L}$ & 0.016 \\
\hline $\mathrm{Ca}$ & $43.8 \mathrm{mg} / \mathrm{L}$ & 2.4 & $\mathrm{pH}$ & 7.72 & 0.17 \\
\hline $\mathrm{Cl}$ & $65.0 \mathrm{mg} / \mathrm{L}$ & 2.1 & $\mathrm{SiO} 2$ & $5.34 \mathrm{mg} / \mathrm{L}$ & 0.27 \\
\hline DSRD & $370 \mathrm{mg} / \mathrm{L}$ & 19 & SO4 & $78.0 \mathrm{mg} / \mathrm{L}$ & 2.4 \\
\hline$F$ & $0.561 \mathrm{mg} / \mathrm{L}$ & 0.030 & Sp Cond & $615 \mu \mathrm{S} / \mathrm{cm}$ & 18 \\
\hline $\mathrm{K}$ & $2.40 \mathrm{mg} / \mathrm{L}$ & 0.22 & $\mathrm{Sr}$ & $291 \mu \mathrm{g} / \mathrm{L}$ & 14 \\
\hline $\mathrm{Mg}$ & $9.75 \mathrm{mg} / \mathrm{L}$ & 0.41 & V & $3.55 \mu \mathrm{g} / \mathrm{L}$ & 1.24 \\
\hline
\end{tabular}

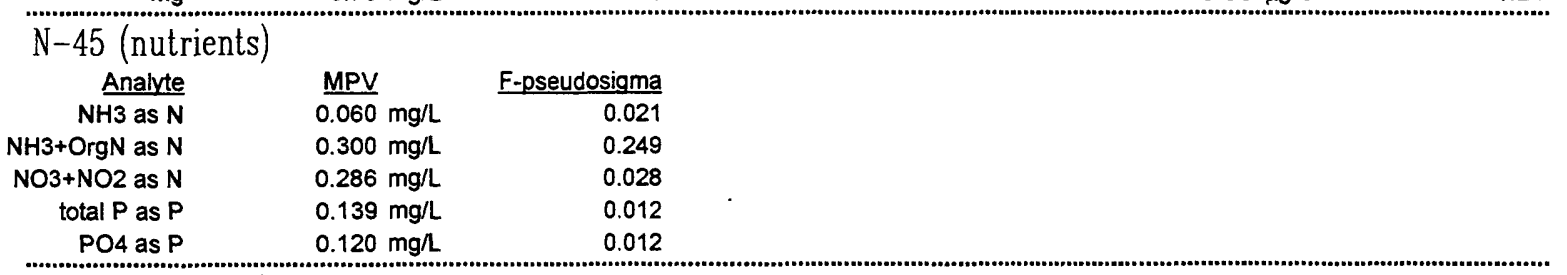

N-46 (nutrients)

$\begin{array}{rrr}\text { Analyte } & \text { MPV } & \text { F-pseudosigma } \\ \mathrm{NH} \text { as } \mathrm{N} & 1.04 \mathrm{mg} / \mathrm{L} & 0.09 \\ \mathrm{NH} 3+\text { OrgN as N } & 1.81 \mathrm{mg} / \mathrm{L} & 0.30 \\ \mathrm{NO}+\mathrm{NO} 2 \text { as N } & 1.23 \mathrm{mg} / \mathrm{L} & 0.06 \\ \text { Total P as } \mathrm{P} & 1.23 \mathrm{mg} / \mathrm{L} & 0.06 \\ \mathrm{PO} \text { 04 as } \mathrm{P} & 0.920 \mathrm{mg} / \mathrm{L} & 0.045\end{array}$

P-24 (low ionic strength constituents)

\begin{tabular}{|c|c|c|c|c|c|}
\hline Analyte & MPV & F-pseudosigma & Analyte & MPV & F-pseudosigma \\
\hline$\overline{\text { Acidity }}$ & $\overline{3.75} \mathrm{mg} / \mathrm{L}$ & 1.30 & $\mathrm{Na}$ & $0.246 \mathrm{mg} / \mathrm{L}$ & 0.025 \\
\hline $\mathrm{Ca}$ & $0.325 \mathrm{mg} / \mathrm{h}$ & 0.030 & $\mathrm{pH}$ & 4.73 & 0.13 \\
\hline $\mathrm{Cl}$ & $1.20 \mathrm{mg} / \mathrm{L}$ & 0.19 & PO4 as $\mathrm{P}$ & $0.028 \mathrm{mg} / \mathrm{L}$ & 0.003 \\
\hline $\mathrm{F}$ & $0.110 \mathrm{mg} / \mathrm{L}$ & 0.011 & SO4 & $0.338 \mathrm{mg} / \mathrm{L}$ & 0.508 \\
\hline K & $0.118 \mathrm{mg} / \mathrm{L}$ & 0.011 & Sp Cond & $13.3 \mu \mathrm{S} / \mathrm{cm}$ & 1.6 \\
\hline $\mathrm{Mg}$ & $0.055 \mathrm{mg} / \mathrm{L}$ & 0.007 & & & \\
\hline \multicolumn{6}{|c|}{$\mathrm{Hg}-2 \mathrm{O}$ (mercury) } \\
\hline Analyte & MPV & F-pseudosigma & & & \\
\hline $\mathrm{Hg}$ & $\overline{4.42} \mu \mathrm{g} / \mathrm{L}$ & 0.38 & & & \\
\hline \multicolumn{6}{|c|}{ SED-5 (bed material) } \\
\hline Analyte & MPV & F-pseudosigma & Analyte & MPV & F-pseudosigma \\
\hline $\mathrm{Ag}$ & $0.72 \mu \mathrm{g} / \mathrm{g}$ & 0.25 & $\overline{\mathrm{Li}}$ & $\overline{3.82} \mu \mathrm{g} / \mathrm{g}$ & 0.98 \\
\hline Al & $4227 \mu \mathrm{g} / \mathrm{g}$ & 979 & $\mathrm{Mg}$ & $1.91 \mathrm{mg} / \mathrm{g}$ & 0.24 \\
\hline As & $216 \mu \mathrm{g} / \mathrm{g}$ & 20.0 & $\mathrm{Mn}$ & $257 \mu g / g$ & 19 \\
\hline B & $9.90 \mu \mathrm{g} / \mathrm{g}$ & 11.82 & Mo & $1.01 \mu \mathrm{g} / \mathrm{g}$ & 0.19 \\
\hline $\mathrm{Ba}$ & $110 \mu \mathrm{g} / \mathrm{g}$ & 14 & $\mathrm{Na}$ & $0.290 \mathrm{mg} / \mathrm{g}$ & 0.031 \\
\hline $\mathrm{Be}$ & $0.305 \mu \mathrm{g} / \mathrm{g}$ & 0.096 & $\mathrm{Ni}$ & $6.68 \mu \mathrm{g} / \mathrm{g}$ & 1.69 \\
\hline $\mathrm{Ca}$ & $8.18 \mathrm{mg} / \mathrm{g}$ & 0.64 & $\mathrm{~Pb}$ & $309 \mu \mathrm{g} / \mathrm{g}$ & 18 \\
\hline $\mathrm{Cd}$ & $158 \mu \mathrm{g} / \mathrm{g}$ & 16 & $\mathrm{Sb}$ & $9.28 \mu \mathrm{g} / \mathrm{g}$ & 3.12 \\
\hline Co & $3.40 \mu \mathrm{g} / \mathrm{g}$ & 0.54 & $\mathrm{Se}$ & $6.69 \mu \mathrm{g} / \mathrm{g}$ & 5.41 \\
\hline $\mathrm{Cr}$ & $11.70 \mu \mathrm{g} / \mathrm{g}$ & 3.20 & $\mathrm{SiO} 2$ & $5.71 \mathrm{mg} / \mathrm{g}$ & 4.16 \\
\hline $\mathrm{Cu}$ & $56.9 \mu \mathrm{g} / \mathrm{g}$ & 25.6 & $\mathrm{Sr}$ & $41.0 \mu \mathrm{g} / \mathrm{g}$ & 5.2 \\
\hline $\mathrm{Fe}$ & $10800 \mu \mathrm{g} / \mathrm{g}$ & 3050 & V & $14.4 \mu \mathrm{g} / \mathrm{g}$ & 5.0 \\
\hline $\mathrm{K}$ & $1.56 \mathrm{mg} / \mathrm{g}$ & 0.29 & $\mathrm{Zn}$ & $598 \mu \mathrm{g} / \mathrm{g}$ & 47 \\
\hline
\end{tabular}

